\title{
Short Sales and Price Discovery in the Hong Kong Stock Market
}

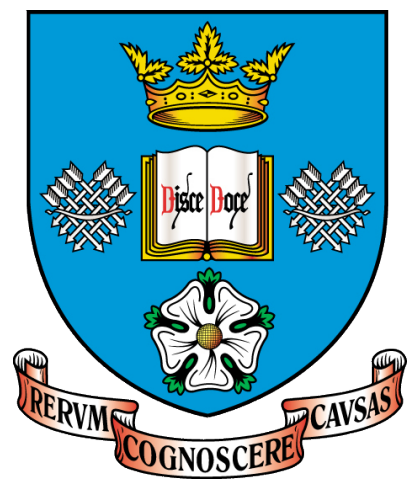

\author{
Wei Xiang \\ Management School \\ University of Sheffield
}

A thesis submitted for the degree of

Doctor of Philosophy

September 2015 



\section{Declaration}

No part of this thesis has been submitted elsewhere for any other degree or qualification in this or any other university. It is all my own work unless referenced to the contrary in the text.

The copyright of this thesis rests with the author. No quotation from it should be published without prior consent and information derived from it should be acknowledged. 

To my parents and beloved wife. 



\section{Abstract}

This thesis explores the effect of short sales on the process of price discovery and the determinants of the speed of price adjustment in the Hong Kong stock market over the period 2001-2011 using an ultra-high frequency dataset.

The thesis extends the VAR model of Hasbrouck (1991) using four estimation methods: OLS and WLS in which the models are estimated on an equation-by-equation basis; VAR and WVAR in which correlations between residuals in both equations are taken into account. To deal with conditional heteroscedasticity present in high frequency data, the thesis also uses GARCH and BEKK models. More detailed analyses cover key determinants of the speed of price adjustment: market conditions, firm size, trading volume and short sellers' activities. The thesis employs a number of non-standard statistical procedures.

The results show that price discovery is affected by short selling for a substantial minority of stocks, but not all. For those affected, short sales accelerate the speed of price adjustment by decreasing the autocorrelations in quote returns and trades. A smaller absolute value of autocorrelation indicates a more efficient process of price discovery. Trade continuity (autocorrelation in quote returns) is affected most when stocks are added to (removed from) the list of stocks eligible for short sales. The results of the more detailed analysis are as follows. Short sales improve price efficiency regardless of market condition. Stocks adjust their prices more quickly in a down market than an up market. The stock prices of larger firms adjust more quickly to new information than smaller ones. Medium-sized (small-sized) firms 
are the most affected when they are added to (removed from) the short selling list. Higher trading volumes are associated with quicker speed of incorporation of new information into prices. Short sellers play an important role in the process of price discovery. 


\section{Acknowledgements}

After all these years of work, this thesis puts an end to the journey to achieve my Doctor of Philosophy degree in Finance which has been a truly life-changing experience. I have not solitarily embarked upon this mission and it is necessary to express my gratitude to those people who help and support me during my PhD study at the University of Sheffield.

First and foremost, I would like to thank my two supervisors, Dr. Shuxing Yin and Professor Chris Adcock, for their continuous patience, structural guidance and cordial words of encouragement through my doctoral study. My work would not be finished without them. What I learned from them in these years become the great treasures and will last a lifetime. I also would like to take this opportunity to thank Professor Jerry Coakley and Dr. John Fry, my viva examiners, for their very helpful and valuable comments and suggestions.

Further, I owe my deepest gratitude to my wife, Tian Jiang, for your commitment, support and endless love. I would not be strong when encountering the bumps and turns along the journey without you. In addition, I would like to thank all friends in Sheffield and faculty staff of Sheffield University Management School for their support.

At last, I want to give my special thanks to my father Professor Tieyuan Xiang and my mother Professor Qiuhua Wang for their full trust and unconditional love over the years. I would not be here without my parents. Dad and Mum, thank you and I love you! 



\section{Contents}

1 Introduction 1

1.1 Overview . . . . . . . . . . . . . . . . . . . 1

1.2 Institutional Background . . . . . . . . . . . . . . . . . . . . 4

1.2.1 Short Sales in Hong Kong . . . . . . . . . . . . . . . . . . . 4

1.2.2 Simultaneous Entry to the Hang Seng Index . . . . . . . . . 5

1.3 Motivations, Research Questions and Summary . . . . . . . . . 6

1.4 Structure of the Thesis . . . . . . . . . . . . . . . . . . . 9

2 Tests of Hypothesis for Short Sales and Price Discovery using the VAR Model 13

2.1 Introduction . . . . . . . . . . . . . . . . . . . . . . . 13

2.2 Literature Review . . . . . . . . . . . . . . . . . . . . . 17

2.3 The Model and Hypothesis Tests . . . . . . . . . . . . . . . . . 20

2.3 .1 The VAR Model . . . . . . . . . . . . . . . . . 20

2.3.2 A Model Consistency Check . . . . . . . . . . . . . . . . . 24

2.3.3 Hypothesis Tests . . . . . . . . . . . . . . . . . . . . 24

2.4 Data Description . . . . . . . . . . . . . . . . 28

2.5 Results . . . . . . . . . . . . . . . . . . . 31

2.5.1 Statistics Summary . . . . . . . . . . . . . 32

2.5.2 Model Validation . . . . . . . . . . . . . . . . . . 33

2.5.3 Changes in Model Parameters . . . . . . . . . . . . . . . 33

2.5.4 Parameter Dynamics . . . . . . . . . . . . . . . . . 37

2.5.5 Z Scores for Tests of Difference in Model Parameters . . . . 38

2.5.6 Model Selection . . . . . . . . . . . . . . . . . . 40

2.6 Conclusions . . . . . . . . . . . . . . . . . . . . . 41

Appendix A Consistency of the VAR Model $\quad 59$ 
Appendix B Test of Significance of $\Omega$ for the VAR Model Consistency 62

$\begin{array}{lll}\text { Appendix C Other Tables } & 64\end{array}$

Appendix D The Example of Data Format 84

3 Short Sales and Price Discovery using GARCH and BEKK Models 86

3.1 Introduction . . . . . . . . . . . . . . . . . . . 86

3.2 The Models and Diagnostic Checking . . . . . . . . . . . . . . . . . 89

3.2.1 The Univariate GARCH Model . . . . . . . . . . . . . . . 89

3.2 .2 The BEKK Model . . . . . . . . . . . . . . . . . . . 91

3.2 .3 Diagnostic Checking . . . . . . . . . . . . . . 94

3.3 Data Description . . . . . . . . . . . . . . . . . . . . . 98

3.4 Results . . . . . . . . . . . . . . . . . . . . . . . . 99

3.4.1 Diagnostic Tests for ARCH Effects . . . . . . . . . . . . . 99

3.4.2 Selection Criteria for Different GARCH Models . . . . . . . 101

3.4.3 Model Validation . . . . . . . . . . . . . . . . . . . . . 102

3.4.4 Changes in Model Parameters . . . . . . . . . . . . . . . . 103

3.4.5 Parameter Dynamics . . . . . . . . . . . . . . . . . 106

3.4.6 Z Scores for Tests of Difference in Model Parameters . . . . 108

3.5 Conclusions ......................... 111

$\begin{array}{lll}\text { Appendix E Other Tables } & 134\end{array}$

4 The Effect of Market Returns, Crises, Market Capitalisation, $\begin{array}{lc}\text { Trading Volume and Short Sales. } & 138\end{array}$

4.1 Introduction . . . . . . . . . . . . . . . . . . . . 138

4.2 Literature Review . . . . . . . . . . . . . . . . . . . . . . . . . 141

4.3 Model for the Effect of Market Conditions . . . . . . . . . . . . . . 144

4.4 Data Description . . . . . . . . . . . . . . . . . . 146

4.5 Results . . . . . . . . . . . . . . . . . . . . . . 148

4.5.1 Market Conditions . . . . . . . . . . . . . . . 148

4.5.1.1 Dummy Variable of Current and One-day Lagged

Market Return . . . . . . . . . . . . . . . . . 148

4.5.1.2 Crisis Period . . . . . . . . . . 153 
4.5.1.3 Up and Down Markets . . . . . . . . . . . . . 155

4.5.2 Market Capitalisation . . . . . . . . . . . . 156

4.5.3 Trading Volume . . . . . . . . . . . . . . . . . . . . 158

4.5.4 Shorting Activity . . . . . . . . . . . . . . . . . . 159

4.5.4.1 Short Interest . . . . . . . . . . . . . . 159

4.5.4.2 Shorting Flow . . . . . . . . . . . . 160

4.6 Conclusions . . . . . . . . . . . . . . . . . . . . 161

$\begin{array}{lll}\text { Appendix F Other Table } & 205\end{array}$

5 Conclusions 207

5.1 Summary of Findings . . . . . . . . . . . . . . . . . . . 207

5.2 Areas for Future Research . . . . . . . . . . . . . . . . . 213

$\begin{array}{ll}\text { References } & 215\end{array}$ 



\section{List of Tables}

Table 1.1 The Records of Addition and Deletion Events of the D-list and the HSI ............................. 11

Table 2.1 The Records of Addition and Deletion Events of the D-list on the HKEx . . . . . . . . . . . . . . . . . . . . . . . . . 43

Table 2.2 Summary Statistics . . . . . . . . . . . . . . . . . . . . . 45

Table 2.3 Model Validation . . . . . . . . . . . . . . . . . . . . . . 47

Table 2.4 Frequency of Changes in Model Parameters for Addition Events (OLS) . . . . . . . . . . . . . . . . . . . 48

Table 2.5 Frequency of Changes in Model Parameters for Deletion Events $(\mathrm{OLS}) \ldots \ldots \ldots \ldots \ldots \ldots$

Table 2.6 Frequency of Changes in Model Parameters for Addition Events $(\mathrm{VAR}) \ldots \ldots \ldots \ldots \ldots \ldots$

Table 2.7 Frequency of Changes in Model Parameters for Deletion Events

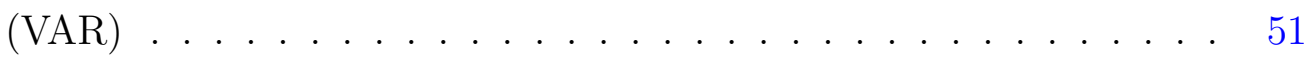

Table 2.8 Summary of the Differences in Frequency of Changes in Model Parameters for Addition Events . . . . . . . . . . . . . 52

Table 2.9 Summary of the Differences in Frequency of Changes in Model

Parameters for Deletion Events . . . . . . . . . . . . 53

Table 2.10 Parameter Dynamics (OLS) . . . . . . . . . . . . . 54

Table 2.11 Summary of Parameter Dynamics . . . . . . . . . . . . 55

Table $2.12 Z$ Scores for Tests of Difference in Model Parameters . . . . . 56

Table 2.13 Residual Correlation Test . . . . . . . . . . . . . . 57

Table 2.14 Summary of Model Estimations . . . . . . . . . . . . 58

Table C.1 Frequency of Changes in Model Parameters for Addition Events $(\mathrm{WLS}) \ldots \ldots \ldots \ldots \ldots \ldots$ 
Table C.2 Frequency of Changes in Model Parameters for Deletion Events

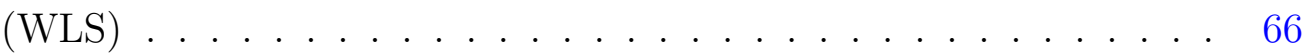

Table C.3 Frequency of Changes in Model Parameters for Addition Events

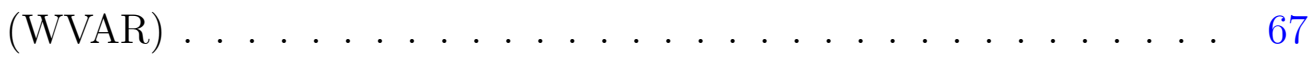

Table C.4 Frequency of Changes in Model Parameters for Deletion Events

(WVAR) . . . . . . . . . . . . . . . . . . 68

Table C.5 Difference in Frequency of Changes in Model Parameters for

Addition Events (OLS vs. WLS) _ . . . . . . . . . . . 69

Table C.6 Difference in Frequency of Changes in Model Parameters for

Deletion Events (OLS vs. WLS) . . . . . . . . . . . . 70

Table C.7 Difference in Frequency of Changes in Model Parameters for

Addition Events (VAR vs. WVAR) . . . . . . . . . . 71

Table C.8 Difference in Frequency of Changes in Model Parameters for

Deletion Events (VAR vs. WVAR) . . . . . . . . . . . . 72

Table C.9 Difference in Frequency of Changes in Model Parameters for

Addition Events (OLS vs. VAR) . . . . . . . . . . . . 73

Table C.10 Difference in Frequency of Changes in Model Parameters for

Deletion Events (OLS vs. VAR) . . . . . . . . . . . . . 74

Table C.11 Difference in Frequency of Changes in Model Parameters for

Addition Events (WLS vs. WVAR) . . . . . . . . . . . 75

Table C.12 Difference in Frequency of Changes in Model Parameters for

Deletion Events (WLS vs. WVAR) . . . . . . . . . . 76

Table C.13 Parameter Dynamics (WLS) . . . . . . . . . . . . . . 77

Table C.14 Parameter Dynamics (VAR) . . . . . . . . . . . . . . . 78

Table C.15 Parameter Dynamics (WVAR) . . . . . . . . . . . . . . . . 79

Table C.16 P-Values for Tests of Model Parameters (OLS) . . . . . . . . 80

Table C.17 P-Values for Tests of Model Parameters (WLS) . . . . . . . . 81

Table C.18 P-Values for Tests of Model Parameters (VAR) . . . . . . . . 82

Table C.19 P-Values for Tests of Model Parameters (WVAR) . . . . . . . 83

Table D.1 The Example of Data Format . . . . . . . . . . . . . . . . 85

Table 3.1 Diagnostic Tests for ARCH effects (OLS Addition Events) . 114

Table 3.2 Diagnostic Tests for ARCH effects (OLS Deletion Events) . . 116 
Table 3.3 Diagnostic Tests for ARCH effects (VAR Addition Events) . 118

Table 3.4 Diagnostic Tests for ARCH effects (VAR Deletion Events) . 119

Table 3.5 Selection Criteria for Different GARCH-type Models (Addition Events) . . . . . . . . . . . . . . . . . 120

Table 3.6 Selection Criteria for Different GARCH-type Models (Deletion Events) . . . . . . . . . . . . . . . . . . . 121

Table 3.7 Model Validation . . . . . . . . . . . . . . . . . . . . . . . . . 122

Table 3.8 Frequency of Changes in Model Parameters for Addition Events $(\mathrm{GARCH}) \ldots \ldots \ldots \ldots \ldots \ldots \ldots$

Table 3.9 Frequency of Changes in Model Parameters for Deletion Events $(\mathrm{GARCH}) \ldots \ldots \ldots \ldots \ldots \ldots \ldots$

Table 3.10 Frequency of Changes in Model Parameters for Addition Events (BEKK) . . . . . . . . . . . . . . . . . . 125

Table 3.11 Frequency of Changes in Model Parameters for Deletion Events (BEKK) . . . . . . . . . . . . . . . . 126

Table 3.12 Comparison on Frequency of Changes in Model Parameters for Addition Events . . . . . . . . . . . . . . . . . . . . . 127

Table 3.13 Comparison on Frequency of Changes in Model Parameters for Deletion Events . . . . . . . . . . . . . . . . . . . . . . . 128

Table 3.14 Parameter Dynamics $(\mathrm{GARCH})$. . . . . . . . . . . . . . . 129

Table 3.15 Parameter Dynamics (BEKK) . . . . . . . . . . . . . . . . . 130

Table 3.16 Summary of Parameter Dynamics . . . . . . . . . . . . . . . 131

Table 3.17 Z Scores for Tests of Difference in Model Parameters (Addition) 132

Table $3.18 Z$ Scores for Tests of Difference in Model Parameters (Deletion) 133

Table E.1 Model Validation . . . . . . . . . . . . . . . . . . . . 135

Table E.2 P-Values for Tests of Model Parameters (GARCH) . . . . . . 136

Table E.3 P-Values for Tests of Model Parameters (BEKK) . . . . . . . 137

Table 4.1 Diagnostic Tests for ARCH effects (OLS - Addition Events Current Market Return Dummy Variable) . . . . . . . . . . . . . . 163

Table 4.2 Diagnostic Tests for ARCH effects (OLS - Deletion Events Current Market Return Dummy Variable) . . . . . . . . . . . . 165 
Table 4.3 Diagnostic Tests for ARCH effects (WLS - Addition Events Current Market Return Dummy Variable) . . . . . . . . . . . . 167

Table 4.4 Diagnostic Tests for ARCH effects (WLS - Deletion Events -

Current Market Return Dummy Variable) . . . . . . . . . . . . . . 169

Table 4.5 Diagnostic Tests for ARCH effects (OLS - Addition Events -

One-day Lagged Market Return Dummy Variable) . . . . . . . . . . 171

Table 4.6 Diagnostic Tests for ARCH effects (OLS - Deletion Events -

One-day Lagged Market Return Dummy Variable) . . . . . . . . . . 173

Table 4.7 Diagnostic Tests for ARCH effects (WLS - Addition Events -

One-day Lagged Market Return Dummy Variable) . . . . . . . . . . 175

Table 4.8 Diagnostic Tests for ARCH effects (WLS - Deletion Events -

One-day Lagged Market Return Dummy Variable) . . . . . . . . . . 177

Table 4.9 P-Value for Tests of Model Parameters (OLS - Dummy Variable of Current Market Return) . . . . . . . . . . . . . . . . . 179

Table 4.10 P-Value for Tests of Model Parameters (WLS - Dummy Variable of Current Market Return) . . . . . . . . . . . . . . 180

Table 4.11 P-Value for Tests of Model Parameters (GARCH - Dummy Variable of Current Market Return) . . . . . . . . . . . . . . . . . 181

Table 4.12 P-Value for Tests of Model Parameters (OLS - Dummy Variable of One-day Lagged Market Return) . . . . . . . . . . . . . . . 182

Table 4.13 P-Value for Tests of Model Parameters (WLS - Dummy Variable of One-day Lagged Market Return) . . . . . . . . . . . . . . . 183

Table 4.14 P-Value for Tests of Model Parameters (GARCH - Dummy

Variable of One-day Lagged Market Return) . . . . . . . . . . . . . 184

Table 4.15 P-Value for Tests of Model Parameters - Crisis (Addition) . . 185

Table 4.16 P-Value for Tests of Model Parameters - Crisis (Deletion) . . 186

Table 4.17 P-Value for Tests of Model Parameters Down and Up Markets (Addition) . . . . . . . . . . . . . . . . . . 187

Table 4.18 P-Value for Tests of Model Parameters Down and Up Markets

(Deletion) . . . . . . . . . . . . . . . . . . . . 188

Table 4.19 P-Value for Tests of Model Parameters - MVRANK (Addition) 189 Table 4.20 P-Value for Tests of Model Parameters - MVRANK (Deletion) 191 Table 4.21 P-Value for Tests of Model Parameters - VORANK (Addition) 193 Table 4.22 P-Value for Tests of Model Parameters - VORANK (Deletion) 195 
Table 4.23 P-Value for Tests of Model Parameters - SIRANK (Addition) 197 Table 4.24 P-Value for Tests of Model Parameters - SIRANK (Deletion) 199 Table 4.25 P-Value for Tests of Model Parameters - SFRANK (Addition) 201 Table 4.26 P-Value for Tests of Model Parameters - SFRANK (Deletion) 203

Table F.1 Cumulative Abnormal Returns Before, During and After Crisis.206 



\section{Chapter 1}

\section{Introduction}

\subsection{Overview}

Short selling refers to the practice of selling stocks without owning them by borrowing them from a lender and then returning them at a future date by purchasing them back in the market at that time. Short sellers can benefit from buying back the borrowed stocks if prices go down but suffer losses if prices go up. Short sales provide an alternative trading option for traders with negative expectation of future performance as traders may face difficulties in selling stocks directly if they have bad news.

Since the US Securities and Exchange Commission (SEC) took emergency regulatory action in respect of short sales, banning short sales in the securities of financial institutions temporarily in the recent global financial crisis, the benefits and costs of this ban have become a topic of debate and discussion among investors, regulators and academics. The common belief is that short sales may be problematic when the market lacks confidence and can be used as a tool to mislead other participants resulting in a downward manipulation. In such circumstances, a manipulator short sells the shares of a company and then spreads rumours about the pessimistic prospects of the company. This could be harmful to the company, investors and the entire market. In order to stabilise the market and rebuild investors' confidence in 
the crisis period, market regulators take various regulatory actions on short sales including restricting naked short selling ${ }^{1}$ but permitting covered short selling or prohibiting short sales of some particular securities such as financial stocks.

However, there are divergent views, particularly from researchers, about the benefits of bans or constraints on short sales. Many researchers argue that the responses of market regulators are unprecedented and the actions to ban or limit short sales are out of line with the objectives and principles of market regulation if regulators aim to ensure an efficient market in the midst of the crisis. It is believed that short selling is the reflection of investors' negative expectation of price changes in the future and not all information will be fully reflected on prices if short sales are banned or restricted. In the presence of short sales constraints price transmissions are asymmetric, which can cause overvaluation (Miller, 1977) and a delay in price adjustment to new information (Diamond \& Verrecchia, 1987). Given heterogeneous beliefs among investors and no restrictions on short sales, Miller (1977) believes that an equilibrium stock price can be jointly set by optimistic investors who buy long and pessimistic investors who sell short. Stocks will be overpriced when there are binding short sales constraints as pessimists are restricted from trading on their beliefs. Many empirical studies find subsequent significant negative stock returns upon the lifting of short sales bans/constraints, which largely support the overvaluation view (Bris et al., 2007; Chang et al., 2007, 2014; Diether et al., 2009; Figlewski, 1981; Jones \& Lamont, 2002).

In the seminal work of Miller (1977), investors are assumed not to acquire information from stock prices. Using a rational expectations model ${ }^{2}$, Diamond \& Verrecchia (1987) provide an alternative view and argue that rational investors can recognise the existence of short sales constraints. Therefore, they are able to adjust the potential bias caused by short sales constraints so that observed stock

\footnotetext{
${ }^{1}$ Unlike traditional (covered) short sales, naked short sales occur when the short seller sells the security first without ever borrowing the security.

${ }^{2}$ People with rational expectations will make decisions based on their rational outlook, all current available information, and past experiences. The current economy expectations are equivalent to what the future economy will be.
} 
prices will represent an unbiased expectation based on all available information and no overpricing of stocks will exist. In addition, their model indicates that the prices of stocks without the eligibility of short sales will have a delayed speed of adjustment to unfavourable private information as short sellers' activities are limited in the market. Due to the lack of data and to difficulties in characterising the speed of price adjustment, only a few papers empirically test the prediction of Diamond \& Verrecchia (1987), namely that short sales constraints slow down the process of price discovery (Beber \& Pagano, 2013; Boehmer \& Wu, 2013; Bris et al., 2007; Chang et al., 2014; Chen \& Rhee, 2010; Saffi \& Sigurdsson, 2010). Another key empirical issue is to determine an appropriate measure of short sales constraints. Most studies use indirect measurements as the proxies for short sales constraints including short interest (Figlewski, 1981); breadth of ownership ${ }^{3}$ (Chen et al., 2002); institutional ownership (Nagel, 2005); costs of short sales (Jones \& Lamont, 2002; Saffi \& Sigurdsson, 2010); option introductions (Danielsen \& Sorescu, 2001). Besides these, Chang et al. (2007) use institutional changes and provide a direct examination on the effect of short sales constraints in the Hong Kong stock market. Stocks in the Hong Kong market are allowed for short sales only when they are added to the designated list (called the D-list). This list is revised on a quarterly basis, with stocks being deleted from it as well as added to it. This rare institutional environment allowed Chang et al. (2007) to trace changes in price movements before and after stocks are added to or removed from the list for short sales.

Taken together, the main purpose of this thesis is to empirically explore the effect of short sales on the process of price discovery as well as the determinants of the speed of price adjustment during both addition and deletion events in the Hong Kong stock market.

\footnotetext{
${ }^{3}$ Breadth is defined as the number of investors with long positions in a particular stock. Lower breadth of a stock is associated with more investors sitting on the sidelines with pessimistic opinions not registered in the stock's price.
} 


\subsection{Institutional Background}

\subsubsection{Short Sales in Hong Kong}

The Hong Kong Stock Exchange [HKEx henceforth] introduced short selling in January 1994. Different from the US market where most of the stocks are shortable, stocks on the HKEx are allowed for short selling only when they are added to the D-list by meeting certain requirements ${ }^{4}$. The selection criteria for inclusion in the D-list for short selling include: $(i)$ all constituent stocks of the underlying indices with financial derivatives written and traded on the HKEx; (ii) underlying stocks of individual stock options and futures; (iii) stocks with a minimum public flotation of HK $\$ 10$ billion, a minimum market capitalisation ${ }^{5}$ of $\mathrm{HK} \$ 3$ billion, or a minimum annual turnover ratio ${ }^{6}$ of $50 \%$. Stocks that meet at least one requirement for at least 3 months can be added to the D-list which is revised quarterly. The HKEx provides daily dissemination of short sales information including daily short selling trading volume.

The HKEx is an order driven market without market markers. Orders are submitted to the Automatic Order Matching and Execution System (AMS) and executed by matching the price and in order of time of arrival. The regulations for stock borrowing and lending require the traders to show their collateral which is a minimum of $105 \%$ of the borrowed stocks' market value. The practise for short sales, except short sales for stock futures, follows the up-tick rule that a short sale cannot be made below the best current ask price. Naked or uncovered short sales without stock borrowing arrangements are forbidden by the exchange and are considered to be a criminal offence. When placing short sales orders in the AMS system, all transactions are identified to the HKEx by the broker and the information is available to the public through the limit order book by flagging the short orders. A ledger with specific details about each short selling order is required to be available

\footnotetext{
${ }^{4}$ The latest selection criteria for short selling is the updated version in July 2012 and full details can be found at http://www.hkex.com.hk/eng/market/sec_tradinfo/regshortsell.htm.

${ }^{5}$ Market capitalisation is the total number of issued shares times the latest share price.

${ }^{6}$ Annual turnover is the aggregate turnover during the preceding 12 months.
} 
to the HKEx at any time.

\subsubsection{Simultaneous Entry to the Hang Seng Index}

The Hang Seng Index [HSI henceforth] was launched on 24 November 1969 and it measures the performance of largest and most liquid companies listed in Hong Kong. The number of constituents is fixed at 50 for the index. To be eligible for selection, a company: $(i)$ must be among those companies that constitute the top $90 \%$ of the total market capitalisation of all primary listed shares on the HKEx (market capitalisation is expressed as an average of the past 12 months); (ii) must be among those companies that constitute the top $90 \%$ of the total turnover of all primary listed shares on the HKEx (turnover is aggregated and individually assessed for eight quarterly sub-periods for the past 24 months); and (iii) should normally have a listing history of 24 months on the HKEx or meet the requirements of the guidelines for handling large-cap stocks listed for less than 24 months. From the many eligible candidates, final selections are based on the following: $(i)$ the market capitalisation and turnover ranking of the company; (ii) the representation of the relevant sub-sector within the HSI directly reflecting that of the market; and ( iii) the financial performance of the company.

[Insert Table 1.1 about here]

Table 1.1 shown above reports the number of changes to the D-list with respect to simultaneous entry to the HSI. As shown in the table, there are 1,296 addition and 810 deletion events during the 10-year study period. Regarding the simultaneous entry to the HSI and the D-list, there is no stock being added to/removed from the D-list and the HSI on the same effective date. Furthermore, there are only 3 out of 1,296 stock/events $(0.23 \%$ ) being added to the HSI around (one month before and after) the effective date of being shortable. Therefore, the effect of entering into the index can be negligible and the changes in the speed of price adjustment 
are mainly attributed to the changes of the D-list not the index.

\subsection{Motivations, Research Questions and Sum- mary}

As discussed earlier, direct evidence on the asymmetric speed of price adjustment is scarcer due to the limitation of data availability and the difficulty of measuring the speed. To fill this gap, Chapter 2 extends a bivariate vector autoregressive [VAR henceforth] model of Hasbrouck (1991) and aims to offer a direct examination of the effect of short sales on the speed of price adjustment based on ultra-high frequency trade-by-trade data over a 10-year period from 2001 to 2011 in the Hong Kong stock market. This VAR approach has been applied to high-frequency data to measure informational content of stock trades and the price efficiency. Chen \& Rhee (2010) use the VAR model containing quote returns and signed trade indicators to measure the respective speed of price adjustment between shortable and non-shortable stocks. They find that stocks have greater autocorrelations in quote returns and less trade continuity indicating a quicker speed of price adjustment when they are added to the short selling list. On the contrary, Boehmer \& Wu (2013) argue that an efficient process of price discovery should follow a random walk in association with a smaller autocorrelations. They find that more shorting flows speed up the incorporation of new information into prices by weakening the autocorrelations in quote returns.

Chapter 2 contributes to the existing literature in the following ways. First, this chapter extends the VAR model by adding a constant to both equations in the model. This modification removes a potential internal inconsistency in the standard implementation. Secondly, different from Chen \& Rhee (2010)'s model estimation on a daily basis, the VAR model in this chapter is estimated for each stock/event based on the transactions in a 60-day period before and after the addition or deletion event. Thirdly, this chapter focusses not only on individual model parameters and summaries thereof, but also on their dynamics. Consideration of 
changes provides deeper insights into the effect of short sales on the process of price discovery during both addition and deletion events. Fourthly, this chapter also consider the variable time duration of consecutive trades during the model estimation as information is conveyed by trades as well as by time (Diamond \& Verrecchia, 1987; Easley \& O'Hara, 1992). Moreover, a bona fide VAR model as well as its time-weighted form are also employed with consideration of cross-sectional correlation of the residuals. Thus, there are four estimation methods: namely ordinary least squares, weighted least squares, VAR and weighted VAR (henceforth denoted OLS, WLS, VAR and WVAR respectively). Finally, the chapter employs a number of non-standard statistical procedures required to deal the effect of unequal stock specific volatility in the hypothesis testing procedures.

The findings from Chapter 2 show that a small number of stocks do exhibit significant changes including both increases and decreases in the magnitude of estimated parameters. The overall results indicate that short sales accelerate the speed of price adjustment by decreasing the autocorrelations in both quote returns and trades in addition events; these findings being consistent with those from Boehmer \& Wu (2013), namely that short sales contribute to a more efficient process of price discovery with a smaller autocorrelations in quote returns which is more associated with a random walk. The study of parameter dynamics reports that there is a great consistency for the majority of model parameters; that is, parameters which, for example, are statistically significantly negative before an event tend to remain in that category after it. Comparing OLS and WLS, it is noted that the VAR and WVAR models have a better ability to capture residual correlations as well as the effect of short sales on model parameters. The WVAR model is considered to be the preferred model as it allows for variable times between trades and is preferable for theoretical reasons.

Following the studies in Chapter 2 and empirical evidence from other studies in financial economics using high frequency data, it is believed that high frequency quotations and trades in the VAR model invariably exhibit heterogeneity of variance. Chapter 3 therefore carries an investigation into the effect of short sales on 
the process of price discovery taking into account time-varying volatility and covariance. The contribution of this chapter is summarised as follows. To the best of our knowledge, the study in Chapter 3 is the first to examine changes in the speed of price adjustment affected by short sales with consideration of heteroscedasticity. The Generalised ARCH [GARCH henceforth] model developed by Bollerslev (1986) is applied to each equation of the VAR model to capture the time-varying variance from individual quote returns or signed volumes. The VAR model is also estimated using the BEKK model of Engle \& Kroner (1995), which takes account of possible volatility spillover between quote returns and signed volumes as well as time-varying volatilities. The BEKK model is both parsimonious and ensures the positive semi-definiteness of the time-varying covariance matrix.

The results of Chapter 3 are as follows. First, compared with the results under the least squares based models of Chapter 2, more stocks exhibit significant changes including both increases and decreases in the absolute value of model parameters under the GARCH and BEKK models. Overall, short sales accelerate the speed of price adjustment when time-varying variances and covariances are considered, but are consistent with the results based on models of Chapter 2. Secondly, combining the results from Chapters $2 \& 3$, it is found that trade continuity (autocorrelation in quote returns) is the most affected during addition (deletion) events. Thirdly, the combined results from six estimation models reveal that the VAR models estimated simultaneously and taking heterogeneous variances and covariances into account (WVAR and BEKK) are more powerful to capture the changes in the speed of price adjustment than the other four models.

The purpose of Chapter 4 is to explore how the speed of price adjustment changes by controlling market condition, firm size, trading volume and short sellers' activities. This chapter makes several contributions. First, it introduces an extension to the VAR model interacted with a dummy variable of current and one-day lagged market returns to examine the speed of price adjustment under different market conditions. Furthermore, the model interacted with the dummy variable under the GARCH framework is also used to deal with the issue of heteroscedasticity. Secondly, the estimation results are divided into two periods, crisis and non-crisis, 
in order to examine the effect of short sales on price efficiency during the extreme market conditions of 2007-2009. Thirdly, the models are re-estimated using by splitting that data into two sets: days in the 60-day period with positive market returns and non-positive market returns, respectively. Lastly, this chapter compares the differences in the speed of price adjustment directly according to stocks' market capitalisation, trading volume and shorting activities.

The findings in Chapter 4 are as follows. Short sales enhance price efficiency regardless of market conditions and stocks are faster in response to new information when the market is bearish. By looking at the recent global financial crisis, it is found that short sales contribute to the efficient process of price discovery only in the non-crisis period. Mixed results during the crisis period indicate that the role of short sales in extreme market conditions is ambiguous. For the size effect, it is reported that large firms react more quickly to new information than small firms during both addition and deletion events. Medium (small) firms are the most affected during the addition (deletion) events. For the volume effect, stocks with more trading volumes are more likely to be affected during the addition events. The results for the deletion events are mixed as it shows that stocks with highest and lowest trading volumes are the most affected ones. For both events, it is noted that stocks with higher trading volumes have faster speed of price adjustment than those with lower trading volumes and the results are consistent under the models when the time duration is not considered. For the effect of short sellers, the results from using short interest and shorting flow as explanatory variables indicate that the speed of price adjustment is faster when short sellers are more active. It therefore supports the view that short sellers play a prominent role to improve price efficiency in the market.

\subsection{Structure of the Thesis}

This thesis consists of four further chapters, as follows. Chapter 2 contains the tests of hypothesis for short sales and price discovery in the Hong Kong stock market 
using the VAR model based on least squares type methods. Chapter 3 explores the effect of short sales by estimating the VAR model under GARCH and BEKK frameworks. Chapter 4 conducts the detailed analysis of the impact of market conditions, firm size, trading volume and short sellers' activities on the speed of price adjustment. Chapter 5 summarises the findings of the thesis and discusses limitations and directions for future research. The tables and the appendices are at the end of each chapter. 


\section{Table 1.1: The Records of Addition and Deletion Events of the D-list and the HSI}

The table reports the revision history of short selling list on the HKEx and the corresponding records for the HSI during the study period of 2001-2011. Columns are as follows: 1. Announcement date; 2. Effective date; 3. No. of additions; 4. No. of deletions; 5 . No. of stocks on the list after each revision; 6 . No. of additions entry to the HSI; 7. No. of deletions quit from the HSI.

\begin{tabular}{cccccccccccccc}
\hline $\mathbf{1}$ & $\mathbf{2}$ & $\mathbf{3}$ & $\mathbf{4}$ & $\mathbf{5}$ & $\mathbf{6}$ & $\mathbf{7}$ & $\mathbf{1}$ & $\mathbf{2}$ & $\mathbf{3}$ & $\mathbf{4}$ & $\mathbf{5}$ & $\mathbf{6}$ & $\mathbf{7}$ \\
\hline $03 / 05 / 2001$ & $14 / 05 / 2001$ & 6 & 0 & 280 & 1 & 0 & $14 / 05 / 2007$ & $21 / 05 / 2007$ & 29 & 14 & 451 & 0 & 0 \\
$07 / 08 / 2001$ & $20 / 08 / 2001$ & 9 & 11 & 278 & 1 & 0 & $18 / 05 / 2007$ & $21 / 05 / 2007$ & 1 & 0 & 452 & 0 & 0 \\
$27 / 11 / 2001$ & $03 / 12 / 2001$ & 17 & 85 & 210 & 0 & 0 & $22 / 05 / 2007$ & $29 / 05 / 2007$ & 1 & 0 & 453 & 0 & 0 \\
$05 / 02 / 2002$ & $25 / 02 / 2002$ & 7 & 14 & 203 & 0 & 0 & $27 / 06 / 2007$ & $04 / 07 / 2007$ & 1 & 0 & 454 & 0 & 0 \\
$08 / 05 / 2002$ & $21 / 05 / 2002$ & 11 & 6 & 208 & 0 & 0 & $16 / 07 / 2007$ & $17 / 07 / 2007$ & 1 & 0 & 455 & 0 & 0 \\
$17 / 07 / 2002$ & $29 / 07 / 2002$ & 24 & 5 & 227 & 0 & 0 & $06 / 08 / 2007$ & $13 / 08 / 2007$ & 134 & 9 & 580 & 0 & 0 \\
$28 / 11 / 2002$ & $29 / 11 / 2002$ & 6 & 15 & 218 & 1 & 0 & $20 / 08 / 2007$ & $27 / 08 / 2007$ & 1 & 0 & 581 & 0 & 0 \\
$21 / 01 / 2003$ & $27 / 01 / 2003$ & 5 & 7 & 216 & 0 & 0 & $19 / 11 / 2007$ & $26 / 11 / 2007$ & 64 & 23 & 622 & 0 & 0 \\
$07 / 05 / 2003$ & $19 / 05 / 2003$ & 18 & 7 & 227 & 0 & 0 & $07 / 12 / 2007$ & $14 / 12 / 2007$ & 2 & 0 & 624 & 0 & 0 \\
$14 / 07 / 2003$ & $21 / 07 / 2003$ & 1 & 16 & 212 & 0 & 0 & $11 / 12 / 2007$ & $14 / 12 / 2007$ & 1 & 0 & 625 & 0 & 0 \\
$27 / 10 / 2003$ & $03 / 11 / 2003$ & 36 & 5 & 243 & 0 & 0 & $11 / 02 / 2008$ & $18 / 02 / 2008$ & 33 & 41 & 617 & 0 & 0 \\
$05 / 01 / 2004$ & $06 / 01 / 2004$ & 1 & 0 & 244 & 0 & 0 & $11 / 03 / 2008$ & $13 / 03 / 2008$ & 1 & 0 & 618 & 0 & 0 \\
$03 / 02 / 2004$ & $10 / 02 / 2004$ & 29 & 3 & 270 & 0 & 0 & $05 / 05 / 2008$ & $13 / 05 / 2008$ & 22 & 47 & 593 & 0 & 0 \\
$06 / 04 / 2004$ & $07 / 04 / 2004$ & 1 & 0 & 271 & 0 & 0 & $14 / 05 / 2008$ & $15 / 05 / 2008$ & 1 & 0 & 594 & 0 & 0 \\
$20 / 04 / 2004$ & $27 / 04 / 2004$ & 26 & 4 & 293 & 0 & 0 & $02 / 06 / 2008$ & $03 / 06 / 2008$ & 5 & 0 & 599 & 0 & 0 \\
$25 / 06 / 2004$ & $01 / 07 / 2004$ & 1 & 0 & 294 & 0 & 0 & $31 / 07 / 2008$ & $07 / 08 / 2008$ & 10 & 51 & 558 & 0 & 0 \\
$05 / 07 / 2004$ & $09 / 07 / 2004$ & 1 & 0 & 295 & 0 & 0 & $07 / 11 / 2008$ & $14 / 11 / 2008$ & 6 & 144 & 420 & 0 & 0 \\
$26 / 07 / 2004$ & $02 / 08 / 2004$ & 8 & 21 & 282 & 0 & 0 & $05 / 02 / 2009$ & $12 / 02 / 2009$ & 25 & 27 & 418 & 0 & 0 \\
$01 / 11 / 2004$ & $08 / 11 / 2004$ & 9 & 11 & 280 & 0 & 0 & $07 / 05 / 2009$ & $14 / 05 / 2009$ & 13 & 22 & 409 & 0 & 0 \\
$28 / 01 / 2005$ & $07 / 02 / 2005$ & 15 & 7 & 288 & 0 & 0 & $03 / 07 / 2009$ & $10 / 07 / 2009$ & 1 & 0 & 410 & 0 & 0 \\
$28 / 02 / 2005$ & $01 / 03 / 2005$ & 2 & 0 & 290 & 0 & 0 & $29 / 07 / 2009$ & $05 / 08 / 2009$ & 49 & 16 & 443 & 0 & 0 \\
$09 / 05 / 2005$ & $17 / 05 / 2005$ & 37 & 9 & 318 & 0 & 0 & $29 / 10 / 2009$ & $05 / 11 / 2009$ & 58 & 11 & 490 & 0 & 0 \\
\hline
\end{tabular}

Table continued on the following page. 
Table 1.1: Continued from previous page

\begin{tabular}{|c|c|c|c|c|c|c|c|c|c|c|c|c|c|}
\hline 1 & 2 & 3 & 4 & 5 & 6 & 7 & 1 & 2 & 3 & 4 & 5 & 6 & 7 \\
\hline $04 / 07 / 2005$ & $08 / 07 / 2005$ & 1 & 0 & 319 & 0 & 0 & $11 / 11 / 2009$ & $18 / 11 / 2009$ & 1 & 0 & 491 & 0 & 0 \\
\hline $11 / 07 / 2005$ & $15 / 07 / 2005$ & 1 & 0 & 320 & 0 & 0 & $30 / 11 / 2009$ & $03 / 12 / 2009$ & 1 & 0 & 492 & 0 & 0 \\
\hline $04 / 08 / 2005$ & $15 / 08 / 2005$ & 14 & 12 & 322 & 0 & 0 & 08/12/2009 & $15 / 12 / 2009$ & 1 & 0 & 493 & 0 & 0 \\
\hline $26 / 08 / 2005$ & $05 / 09 / 2005$ & 1 & 0 & 323 & 0 & 0 & $21 / 12 / 2009$ & $24 / 12 / 2009$ & 1 & 0 & 494 & 0 & 0 \\
\hline $24 / 10 / 2005$ & $28 / 10 / 2005$ & 1 & 0 & 324 & 0 & 0 & $25 / 01 / 2010$ & $01 / 02 / 2010$ & 65 & 8 & 551 & 0 & 0 \\
\hline $18 / 11 / 2005$ & $17 / 11 / 2005$ & 11 & 7 & 328 & 0 & 0 & $22 / 02 / 2010$ & $01 / 03 / 2010$ & 1 & 0 & 552 & 0 & 0 \\
\hline $14 / 02 / 2006$ & $20 / 02 / 2006$ & 10 & 8 & 330 & 0 & 0 & $03 / 03 / 2010$ & $10 / 03 / 2010$ & 1 & 0 & 553 & 0 & 0 \\
\hline $27 / 02 / 2006$ & $01 / 03 / 2006$ & 2 & 0 & 332 & 0 & 0 & $18 / 03 / 2010$ & $25 / 03 / 2010$ & 1 & 0 & 554 & 0 & 0 \\
\hline $19 / 05 / 2006$ & $29 / 05 / 2006$ & 23 & 17 & 338 & 0 & 0 & $03 / 05 / 2010$ & $10 / 05 / 2010$ & 59 & 12 & 601 & 0 & 0 \\
\hline $29 / 05 / 2006$ & $02 / 06 / 2006$ & 1 & 0 & 339 & 0 & 0 & $12 / 07 / 2010$ & $16 / 07 / 2010$ & 1 & 0 & 602 & 0 & 0 \\
\hline $01 / 06 / 2006$ & $02 / 06 / 2006$ & 1 & 0 & 340 & 0 & 0 & $28 / 07 / 2010$ & 04/08/2010 & 40 & 19 & 623 & 0 & 0 \\
\hline $18 / 08 / 2006$ & $25 / 08 / 2006$ & 38 & 10 & 368 & 0 & 0 & $23 / 08 / 2010$ & $30 / 08 / 2010$ & 1 & 0 & 624 & 0 & 0 \\
\hline $24 / 08 / 2006$ & $01 / 09 / 2006$ & 1 & 0 & 369 & 0 & 0 & $25 / 10 / 2010$ & $29 / 10 / 2010$ & 47 & 18 & 653 & 0 & 0 \\
\hline $19 / 10 / 2006$ & $23 / 10 / 2006$ & 1 & 0 & 370 & 0 & 0 & $11 / 11 / 2010$ & $15 / 11 / 2010$ & 1 & 0 & 654 & 0 & 0 \\
\hline $19 / 10 / 2006$ & $27 / 10 / 2006$ & 1 & 0 & 371 & 0 & 0 & $17 / 11 / 2010$ & $22 / 11 / 2010$ & 2 & 0 & 656 & 0 & 0 \\
\hline $24 / 11 / 2006$ & $01 / 12 / 2006$ & 55 & 9 & 417 & 0 & 0 & $17 / 12 / 2010$ & $20 / 12 / 2010$ & 1 & 0 & 657 & 0 & 0 \\
\hline $26 / 02 / 2007$ & $05 / 03 / 2007$ & 30 & 24 & 423 & 0 & 0 & $23 / 12 / 2010$ & $30 / 12 / 2010$ & 1 & 0 & 658 & 0 & 0 \\
\hline $13 / 03 / 2007$ & $14 / 03 / 2007$ & 1 & 0 & 424 & 0 & 0 & $12 / 01 / 2011$ & $28 / 01 / 2011$ & 1 & 0 & 659 & 0 & 0 \\
\hline $12 / 04 / 2007$ & $19 / 04 / 2007$ & 5 & 0 & 429 & 0 & 0 & $25 / 01 / 2011$ & $01 / 02 / 2011$ & 1 & 0 & 660 & 0 & 0 \\
\hline $25 / 04 / 2007$ & $26 / 04 / 2007$ & 6 & 0 & 435 & 0 & 0 & $18 / 02 / 2011$ & $25 / 02 / 2011$ & 70 & 17 & 713 & 0 & 0 \\
\hline $25 / 04 / 2007$ & $27 / 04 / 2007$ & 1 & 0 & 436 & 0 & 0 & $17 / 05 / 2011$ & $24 / 05 / 2011$ & 65 & 18 & 760 & 0 & 0 \\
\hline
\end{tabular}




\section{Chapter 2}

\section{Tests of Hypothesis for Short Sales and Price Discovery using the VAR Model}

\section{$2.1 \quad$ Introduction}

The impact of short sales on market efficiency is a much debated topic in finance and the debate has been reinvigorated by the financial crisis of 2007-09. Many countries $^{7}$ and capital markets around the world imposed restrictions during that period, or even banned short selling (Beber \& Pagano, 2013). In a bear market, short sellers are criticised for exacerbating the market decline, causing market panics and manipulating stock prices. Regulators believe that stricter regulations on short sales can help to protect the market's integrity and quality and rebuild investors' confidence. In sharp contrast, many researchers voice opposition to short selling restrictions. They believe that short selling is the reflection of some market participants' negative expectation of future price changes. In the presence of short sales constraints, price transmissions are asymmetric, which can cause overvalua-

\footnotetext{
${ }^{7}$ After Lehman Brother's bankruptcy in late September 2008, short sales on financial stocks are temporarily prohibited in the United States (from September to October in 2008) and in the United Kingdom (from September 2008 to January 2009), respectively. Countries such as Germany, Spain and France also adopted temporary short sales bans in that period.
} 
tion (Miller, 1977) and a delay in price adjustment to new information (Diamond \& Verrecchia, 1987). Existing studies tend to focus on the relationship between short sales and overvaluation (Bris et al., 2007; Chang et al., 2007, 2014; Diether et al., 2009; Figlewski, 1981; Jones \& Lamont, 2002). However, only a few papers examine the speed of price adjustment to new information. This is apparently due to the lack of data and to difficulties in characterising the speed of price adjustment. An important paper concerned with price adjustment is due to Chen \& Rhee (2010) who use a bivariate VAR model (Hasbrouck, 1991) to measure the respective speed of price adjustment between shortable and non-shortable stocks. Their model includes quote-midpoint changes and signed trade indicators, which are used to describe autocorrelations in quote revision and trade continuity respectively. They find that shortable stocks have a faster speed of price adjustment to new information by exhibiting more negative autocorrelation in mid-quote returns and less positive trade continuity. Boehmer \& Wu (2013) define the mid-quote return as the return on the mid-price for two successive quotations in the VAR model to capture price efficiency and they believe that an efficient price process should follow a random walk. Their results show that a greater shorting flow (that is, a larger number of short selling orders) is associated with smaller pricing errors and smaller mid-quote return autocorrelations, which indicate faster price discovery.

This chapter extends the VAR model of Hasbrouck (1991) and offers a direct examination of the impact of short sales on the speed of price adjustment to new information using high-frequency data over a 10-year period from May 2001 to May 2011 on the HKEx. The HKEx only allows stocks which meet certain requirements to be available for short selling. Furthermore, stocks may be added to and deleted from the short selling list (known as the D-list), which is revised on a quarterly basis. Addition and deletion events may occur multiple times for a given stock during the study period. The D-list and the implied set of addition and deletion events offers the means to examine the effect of short-selling on price discovery.

This chapter also makes six methodological contributions to the existing literature. First, the changes in quotes and trades dynamics in the bivariate VAR model of Hasbrouck (1991) capture the information revealed by trades. The model has been 
widely used to examine the effects of trade-related information (Chen \& Rhee, 2010; Chung et al., 2005; Dufour \& Engle, 2000). This chapter notes an internal inconsistency in the standard implementation of the VAR model which may lead to a violation of its assumptions. To solve this potential problem, the VAR model is modified by adding a constant term to both equations. Secondly, Chen \& Rhee (2010) estimate the VAR model on a daily basis. Examination of the data used in this chapter reveals that during the 60-day period before and after addition and deletion events over a 10-year study period, there are respectively 22,246 days out of 124,202 days (17.9\%) and 30,566 days out of 78,338 days (39.0\%) with less than 20 transactions. Estimating a small number of transactions on a daily basis could cause multi-collinearity and other statistical problems. Therefore, in this chapter, and differently from Chen \& Rhee (2010), the VAR model is estimated for each stock for each addition and deletion event using all transactions in a 60-day period both before and after the events. Thirdly, Chen \& Rhee (2010) focus on the estimated coefficients for lagged quote revisions and signed trade indicators. This chapter analyses the dynamics not only of the individual parameters but also the sums of parameters in the model. This gives a clearer and more detailed picture of how the price discovery process is affected when a stock is either added to or deleted from the D-list. Fourthly, this chapter also examines the informational role of trades by taking consideration of the time duration between two consecutive transactions. Information is conveyed by trades as well as by time (Diamond \& Verrecchia, 1987; Easley \& O'Hara, 1992). Informed traders will always trade unless they do not have stocks and information or there are short-sale constraints in the market. Therefore, it is reasonable to assume that variations of trade time duration are associated with the behaviour of informed traders. Using the standard assumption that volatility is proportional to the square root of the length of a time interval provides a better understanding of the information impact of a trade on price. Fifth, in addition to the use of least squares and weighted least squares on an equation by equation basis, this chapter also uses the bona fide VAR model in which the cross-sectional correlation of the residual terms is considered. Lastly, hypothesis tests in this chapter explicitly recognize that the estimated standard errors of comparable estimated coefficients in the VAR model for different stocks are not the same. They are proportional to the estimated residual or stock specific standard errors. Tests of significance based on standard $t$-tests are therefore not 
appropriate as they are not robust to variance heterogeneity and may lead to misleading inferences. Instead this chapter uses procedures based on a robust version of the $t$-test originally due to Scott \& Smith (1971) [SS test henceforth].

The main results are summarised as follows. First, different from those in Chen \& Rhee (2010), there is a significant decrease (increase) in quote autocorrelations which measure the speed of price adjustment for stocks during the addition (deletion) events. An efficient price process implies that return of quote midpoints follow a random walk, thus a smaller absolute value of autocorrelations caused by short sales indicates a faster speed of price adjustment. Quote revision autocorrelations, which are used as a proxy for speed of price adjustment in the previous empirical studies (Boehmer \& Wu, 2013; Chen \& Rhee, 2010), are observed to decrease (increase) during the addition (deletion) events. Chen \& Rhee (2010) suggest that stronger quote reversals led by weaker trade continuity reveal a quicker speed of price adjustment to new information while Boehmer \& Wu (2013) argue that weaker autocorrelations in quote revision indicates greater price efficiency under random walk theory. In this chapter, the autocorrelations in quote revision become weaker (stronger) for addition (deletion) events and this finding on speed of price adjustment is consistent with those from Boehmer \& Wu (2013). It is also found that trade continuity becomes weaker when stocks are allowed for short selling. This suggests that, in general, short sales contribute to price discovery for addition and deletion events. Secondly, a study of the dynamics indicates that parameters in the model generally remain consistent as far as sign is concerned; that is, an estimated model parameter which is significantly negative (positive) before the addition/deletion event will remain negative (positive) after the event. Thirdly, the results of statistical test on the difference in frequency of changes suggest that significant changes in the price impact of trades are less visible when time durations are considered in the estimation process and more significant changes in the autocorrelations in quote returns and trades are captured if two equations in the model are estimated simultaneously by VAR or by WVAR. Finally, it is found that WVAR model with consideration of time duration between trades has a stronger ability to capture the residual correlations in the model and the effect of short sales 
on price efficiency during the addition and the deletion events.

The chapter is organised as follows. Section 2.2 presents a brief literature review of short sales and their impact on the speed of price adjustment. Section 2.3 introduces the extended version of the VAR model and the non-standard hypothesis tests that are used in the chapter. Section 2.4 describes the data in this chapter as well as the data filtering procedures. The results are discussed in Section 2.5. Section 2.6 summarises the main conclusions and planned further work. There are three separate appendices. Appendices A and B contain details of the model and a related statistical test. Appendix $\mathrm{C}$ contains detailed tables of results.

\subsection{Literature Review}

The effect of short selling on stock prices is debated by regulators, market participants and researchers alike. Short selling has been blamed by regulators and the media for triggering or exaggerating market declines especially during a financial crisis. However, theoretical research, including that of Miller (1977) and Diamond \& Verrecchia (1987), questions the effectiveness of the regulations of short-sales constraints and bans and argue that short selling has a positive impact on stock market prices. This section presents a short review of the impact of short-sale constraints on stock price efficiency and the empirical evidence.

In an efficient price discovery process, stock prices fully reflect all current and past information and adjust to new information instantaneously (Fama, 1991). However, short sale constraints hinder the participation of investors with pessimistic opinions in the market and cause an asymmetric price transmission process. Miller (1977) therefore argues that stock prices have a tendency to reflect a more optimistic view which can lead to overvaluation. Diamond \& Verrecchia (1987) suggest that short sales constraints lead to slower speed of price adjustment to new information, especially to bad news. 
Direct evidence on the asymmetric speed of price adjustment is scarcer owing to the limitation of data availability and the difficulty of measuring the speed. By using differences in short selling regulations globally as different short selling constraints, Bris et al. (2007) use cross-sectional and time-series information in 46 stock markets around the world with two measures for price efficiency. The first measure, following Morck et al. (2000), is to calculate the difference in the $R^{2}$ coefficients (a downside-minus-upside $R^{2}$ ) measured as the relative co-movement of an individual stock with the market, depending on the sign of the market returns on weekly basis. The second measure is based on the cross-autocorrelations between individual stock returns and the signed market returns with 1-week's lag. Bris et al. (2007) find that the downside $R^{2}$ decreases more in countries where short sales are practised, which causes a drop in the difference in the downside-minus-upside $R^{2}$. They also find short sales decrease the total cross-autocorrelation and make them significantly lower for dual-listed stocks. This accords with the hypothesis that there is a faster price discovery for dual-listed stocks which may be shortable in another market (Foerster \& Karolyi, 1999) or when there is no market friction (Hou \& Moskowitz, 2005). Following Bris et al. (2007)'s approaches, Beber \& Pagano (2013) explore short sales regulations in 30 countries during the 2007-09 financial crisis. They also find that the speed of price adjustment is slowed down by short-sales bans, especially when the market is bearish in the 30 countries covered in their study.

Different from Bris et al. (2007), Saffi \& Sigurdsson (2010) use lending supply and loan fees as more precise proxies for short sales restrictions to explain crosssectional differences in price efficiency from 26 countries. These two short-sales proxies overcome the problems caused by country-level information and enable them to examine the role of short selling even in countries without short-sales constraints. In addition to the cross-autocorrelation applied in Bris et al. (2007), Saffi \& Sigurdsson (2010) include the variance ratios of stocks returns (Lo \& MacKinlay, 1988) and two measures of price delay (Hou \& Moskowitz, 2005) which are based on the delay of price-response between stock returns and the lagged returns of a local market index as well as the contemporaneous world index returns. Saffi \& Sigurdsson (2010) confirm that a high level of lending supply and small loan fees are related to an increase in the speed of price adjustment to the information 
and suggest that the implication of short-sales constraints destroy price efficiency, which stands against to the decisions of market regulators.

Going beyond the previous work summarised above, Boehmer \& Wu (2013) add two different measures on a higher frequency basis, the pricing error and the absolute value of intraday return autocorrelations, to capture the relative informational efficiency of prices. The pricing error in Hasbrouck (1993)'s VAR model captures the difference between the actual price and the fundamental value. The quote midpoints, considered as the market's best estimates of the equilibrium value of the stock at the times, should follow a random walk with smaller autocorrelation in quote returns if the price discovery is efficient. Following Chordia et al. (2005), the absolute value of return autocorrelations is estimated at a 30-minute interval. As a result, they conclude that NYSE-listed stocks with a greater flow of short sales have a smaller pricing error and that returns follow a random walk more closely. This indicates that price discovery process is more efficient when short sellers are more active in the market.

Based on the trade-by-trade high frequency data over a 3-year period, Chen \& Rhee (2010) estimate Hasbrouck (1991)'s VAR model daily for the 3-month period before and after the addition or deletion event to test the speed of price adjustment to new firm-specific information in the Hong Kong stock market. The HKEx offers an ideal setting where short selling constraints are directly captured by their add/remove status on the D-list based on certain selection criteria. Chen \& Rhee (2010) believe that stronger positive trade continuity is interpreted as a slower adjustment to new information (Hasbrouck, 1991; Hasbrouck \& Ho, 1987) while weaker negative quote reversal is considered as a delayed price adjustment due to less revision in beliefs accompanied by greater positive correlated order flow (Madhavan et al., 1997). Contrary to Boehmer \& Wu (2013), in the context of price adjustment speed, Chen \& Rhee (2010) argue that more negative autocorrelation in quote returns reflects a faster speed of price adjustment to new information. In their results, they point out that quote reversals increase and trade autocorrelations decrease significantly and simultaneously when stocks are allowed for short selling. In addition, Chen \& Rhee (2010) employ Dimson (1979)'s beta regression 
to compare the speed of price adjustment to market-wide information between shortable and non-shortable stocks. Their findings provide evidence that shortable stock responds faster not only to firm-specific but also market-wide information.

\subsection{The Model and Hypothesis Tests}

This section has three parts. Section 2.3.1 describes the bivariate VAR model used in the chapter and defines the variables that are used. It then summarises the four versions of the model that are considered in this chapter. Section 2.3.2 describes a test that is used to check the internal consistency of the VAR model. Section 2.3.3 describes the various tests of hypothesis that are carried out.

\subsubsection{The VAR Model}

This chapter uses a bivariate VAR model to examine the speed of price adjustment to new information and to contrast the speed before and after the change in stock shortable status. Hasbrouck (1991) reports that transactions are informative and can cause a persistent and permanent impact on prices. The information in a new trade contains the revised beliefs of traders about prices. It thus provides the opportunity for trades to adjust prices accordingly. In conventional regression notation and for a given stock the model used in this chapter is

$$
\begin{aligned}
& R_{t}=\alpha_{0}+\sum_{i=1}^{5} \alpha_{i} R_{t-i}+\sum_{i=0}^{5} \beta_{i} X_{t-i}+\varepsilon_{R t} \\
& X_{t}=\gamma_{0}+\sum_{i=1}^{5} \gamma_{i} R_{t-i}+\sum_{i=1}^{5} \delta_{i} X_{t-i}+\varepsilon_{X t} .
\end{aligned}
$$


In applications reported in the literature, the VAR model used in this chapter and the following chapters is truncated at five lags as in Hasbrouck (1991) and Chen \& Rhee (2010). Thus it includes the constants and that gives 23 parameters in all in the model. Hasbrouck (1991) determines the number of lags by examining autocorrelations and cross-autocorrelations between quote returns and trades. The results show that the coefficients of higher lags are not statistically significant and five is chosen as the optimal lag length as a practical empirical matter. Different from both Hasbrouck's and Chen and Rhee's in that the model in this chapter includes a constant term in each equation. This is because the model without constant terms can lead to internal inconsistencies. These may be removed by the inclusion of constant terms, which also leads to a statistical test for the consistency of the model. Study of the empirical results in both Hasbrouck (1991) and Chung et al. (2005), neither of whom include constants, shows that there are inconsistencies in their models. Details are available in Appendix A. It is not possible to check model consistency for Chen \& Rhee (2010) as they do not report the necessary level of details. The concept of model consistency may not be applied to Dufour \& Engle (2000) as they use a different model. The statistical test, which is also in part a specification test for the model, is described in Section 2.3.2 below.

The variable denoted $R$ is the difference in the natural logarithm of the mid-quotes for two successive transactions.

$$
R_{t}=\ln M_{t}-\ln M_{t-1}, M_{t}=\left(Q_{t}^{b}+Q_{t}^{a}\right) / 2,
$$

where $Q_{t}^{b, a}$ are the bid and ask quotes at time $t$. This so-called quote return will be referred to in the rest of this chapter as return. The variable denoted $X$ is a trade measure. The measure used in this chapter is the signed volume of the trade. According to Hasbrouck (1991), trade $X_{t}$ takes place after bid and ask quotes $Q_{t-1}^{b, a}$. The new bid and ask quotes $Q_{t}^{b, a}$ will be revised based on the occurred trade $X_{t}$ and then more trades will follow. Hence, the variables $Q_{t}^{b, a}$ and $X_{t}$ are not determined simultaneously even though $Q_{t}^{b, a}$ and $X_{t}$ carry the same time subscript 
t. The classification of Lee \& Ready (1991) is used to define the trade sign. A buyer initiated trade is assigned a value of one if the trade price is higher than the prevailing mid-quote price before the trade. A seller initiated trade is assigned a value of minus one if the trade price is lower than the prevailing mid-quote price. If the trade price equals the prevailing mid-quote price but is higher (lower) than the previous trade price, it is classified as buyer-initiated (seller-initiated). If the trade price equals both the prevailing mid-quote price and the previous trade price, the trade sign is undetermined and is assigned a value of zero ${ }^{8}$. For convenience, the quote return variable denoted $R$ is rescaled by multiplying $10^{3}$ and the trade variable denoted $X$ is rescaled by dividing by $10^{4}$.

In the VAR model described above at (2.1) the coefficients $\alpha_{i}$ and $\delta_{i}$ are autocorrelations in quote revision and signed trade volume respectively. The coefficients $\beta_{i}$ indicate the impact on quote revision subsequent to each trade and the coefficient $\beta_{0}$ allows the immediate impact of contemporaneous trade on the quote revision. The coefficients $\gamma_{i}$ capture Granger causality of lagged quote revision on trades. The structure of the non-standard VAR model ${ }^{9}$ assumes that the market contains not only information on all lagged returns and lagged trades but also information on contemporaneous trades available at time $t$. The residual term $\varepsilon_{R}$ in the return equation at (2.1) represents the non-trade public information, while $\varepsilon_{X}$ in the trade equation represents private information from unexpected trades. The non-standard VAR model with the current trade variable in the return equation captures the whole process of how private and public information is incorporated into asset prices gradually through trades by the two residual terms $\varepsilon_{R}$ and $\varepsilon_{X}$.

This chapter considers four versions of the VAR model. First, the general assumption is that the 2 -vector of residuals $\varepsilon_{t}^{T}=\left(\varepsilon_{R, t}, \varepsilon_{X, t}\right)$ has a bivariate normal

\footnotetext{
${ }^{8}$ Alternative trade sign measure, which is used in some papers (Chen \& Rhee, 2010; Chung et al., 2005; Dufour \& Engle, 2000) but not here, is that $X$ is a discrete variable taking values equal to \pm 1 or 0 .

${ }^{9}$ By contrast, the standard VAR model contains no contemporaneous dependent variables but only lags of the dependent variables and the number of lags are the same.
} 
distribution with zero mean vector and constant covariance matrix with no serial correlation. It is further assumed in this chapter that the contemporaneous correlation between $\varepsilon_{R}$ and $\varepsilon_{X}$ is zero. In this case the parameters of the two models may be estimated separately by OLS. Analogous to the market model, it is also assumed that there is no residual correlation between stocks. Secondly, an innovation adopted in this chapter is to take into account the fact that the time intervals between successive trades are not equal. In general, variations in the trade duration could provide information to market participants. Long durations are presumably due to bad news or no news (Diamond \& Verrecchia, 1987; Easley \& O'Hara, 1992) while trade durations are likely associated with the underlying intuition that informed investors always trade as quickly as possible and as much as possible once they have available information (Easley \& O'Hara, 1992). Putting more (less) weight to the trades with less (more) time duration captures the speed of price adjustment to new information at an alternative horizon with explanatory power of inter-trade time. This chapter therefore adopts the standard assumption that volatility is proportional to the square root of the time interval. Variables in the model, quote revisions and signed trade volume are weighted by the reciprocal of $\sqrt{\Delta t}$, the square root of the inter-trade time durations, thus resulting in weighted least squares, denoted by WLS.

The chapter also includes results based on two bona fide VAR models, in which contemporaneous correlation between $\varepsilon_{R}$ and $\varepsilon_{X}$ is allowed. The two models are estimated on an unweighted and weighed basis as described above and are denoted VAR and WVAR respectively.

The parameters in the equations for $R$ and $X$ are referred collectively as $\Theta$. Estimated parameters are denoted as $\hat{\alpha}_{0}$ and so on, collectively $\hat{\Theta}$. A subscript $j$ is used to denote an individual stock. Before and after an addition or deletion event are denoted with subscripts $B$ and $A$. These are used only when required. The estimated covariance matrix of $\hat{\Theta}$ is denoted by $\Sigma_{\hat{\Theta}}$. Sub-matrices of $\Sigma_{\hat{\Theta}}$ which are required for some of the tests described below are denoted $\Sigma_{\hat{\alpha} \hat{\alpha}}$ and so on. 


\subsubsection{A Model Consistency Check}

A consistency check for the fitted model may be derived by taking expected values of the individual equations in (2.1) as described in Appendix B. After rearrangement and elimination of the expected value of $X$, this gives

$$
\Omega=\left\{1-\sum_{i=1}^{5} \alpha_{i}-\left[\left(\sum_{i=0}^{5} \beta_{i}\right)\left(\sum_{i=1}^{5} \gamma_{i}\right) /\left(1-\sum_{i=1}^{5} \delta_{i}\right)\right]\right\} \mu_{R}-\alpha_{0}-\gamma_{0}\left(\sum_{0}^{5} \beta_{i}\right) /\left(1-\sum_{i}^{5} \delta_{i}\right)=0,
$$

where $\mu_{R}$ is the unconditional expected value of $R$. Given the assumptions of the model, the OLS estimates of the parameters are also maximum likelihood (ML) estimates. The ML estimate of $\Omega$ is given by substituting the estimates of the elements of $\Theta$ in equation (2.2). The asymptotic variance of $\hat{\Omega}$ is given using standard methods, as described in Appendix B. The null hypothesis $H_{0}: \Omega=0$ may be tested by the $Z$ statistic

$$
\hat{\Omega} / \sqrt{\operatorname{var}(\hat{\Omega})},
$$

which has a standard normal distribution under the null hypothesis. Rejection of this hypothesis would lead to concerns about the specification of the VAR model at (2.1).

\subsubsection{Hypothesis Tests}

The objective of research in this area is to test the parameters in the model for statistical significance. In addition to standard regression tests, it is important to determine whether the events of addition to or deletion from the short selling list result in statistically significant changes to the model parameters. For example, the null hypothesis for one such test would be 


$$
H_{0}: \alpha_{j, \text { After }}=\alpha_{j, \text { Before }}
$$

along with a suitable alternative, which may be either one or two-sided. A subscript $j$ is used to denote an individual stock. Similar tests are performed for the other parameters in the model at (2.1). If the null hypothesis is rejected, the inference that may be drawn is that the ability or inability to short sell a stock results in changes to the process of price discovery. In addition, it is also common practice to test for the significance of linear combinations of model parameters, namely

$$
A=\sum_{i=1}^{5} \alpha_{i}, B=\sum_{i=0}^{5} \beta_{i}, \Gamma=\sum_{i=1}^{5} \gamma_{i}, \Delta=\sum_{i=1}^{5} \delta_{i}
$$

and to test differences before and after the event. Both of these types of test are complicated by the fact that the residual variance may also be changed by the event. It is therefore unwise to use standard $t$-tests which assume a constant variance.

In addition to tests of the parameters for an individual stock and a single event, it is also normal practice to examine the sums (or equivalently averages) of each model parameter before and after addition and deletion events. Thus, for $n$ stocks, equation (2.4) becomes

$$
H_{0}: \sum_{j=1}^{n} \alpha_{j, A f t e r}=\sum_{j=1}^{n} \alpha_{j, \text { Before }}
$$

The corresponding null hypothesis for the linear combination, the sums, denoted as $A$ in (2.5) is 


$$
H_{0}: \sum_{j=1}^{n} A_{j, A f t e r}=\sum_{j=1}^{n} A_{j, \text { Before }}
$$

with similar hypotheses for the other combinations. Again, standard $t$-tests may not be used because, even under the assumptions of the model at (2.1), the estimated variances of the elements of $\hat{\Theta}$ are different for each stock.

\section{Tests of Single Parameters}

In this section and the next, to simplify the notation an arbitrary parameter in the regression model at (2.1) is denoted by $\alpha$ and its standard deviation by $\sigma$. Estimated parameters are denoted with the ${ }^{\wedge}$ symbol. The standard test of $H_{0}$ : $\alpha=0$ against one or two-sided alternatives is the $t$-test

$$
t=\sqrt{n} \overline{\hat{\alpha}} / \overline{\hat{\sigma}}
$$

An exercise that is carried out in other papers in this area of research (Chen \& Rhee, 2010) is to examine the parameter $\alpha$ using tests based on the estimated values $\hat{\alpha}_{j}$. Loosely, this is to investigate if the mean value of $\alpha$ is significantly different from zero. Using the notation

$$
\overline{\hat{\alpha}}=n^{-1} \sum_{j=1}^{n} \hat{\alpha}_{j}, \overline{\hat{\sigma}}^{2}=(n-1)^{-1} \sum_{j=1}^{n}\left(\hat{\alpha}_{j}-\overline{\hat{\alpha}}\right)^{2}
$$

the usual $t$-test is $t=\sqrt{n} \overline{\hat{\alpha}} / \overline{\hat{\sigma}}$, for which the null hypothesis is $H_{0}: \alpha=0$. The implicit assumption in this test is that the alternative hypothesis is $H_{1}: \alpha \neq 0$. This implies in turn that a non-zero value of $\alpha$ is constant for all stock/events, 
which is a strong assumption. It is, for example, equivalent to assuming that all betas are equal in the market model. It also seems to be an exercise of limited value since the tests carried out under (2.8) invariably indicate that some stock/events have an estimated $\alpha$ that is not significantly different from zero. In addition to these difficulties, the standard $t$-test above assumes that the variance of $\hat{\alpha}_{j}$ is a constant over all stock/events. Since $\sigma$ is proportional to the residual volatility for the stock/event, it is clear that this assumption will be violated. A more formal test uses the property that the estimated variance of $\overline{\hat{\alpha}}$ is

$$
\operatorname{var}(\overline{\hat{\alpha}})=n^{-2} \sum_{j=1}^{n} \hat{\sigma}_{j}^{2} .
$$

Following Scott \& Smith (1971), a test statistics for $H_{0}: \alpha=0$ is

$$
Z=\overline{\hat{\alpha}} / \sqrt{\operatorname{var}(\overline{\hat{\alpha}})}
$$

which is compared to the critical values of the standard normal distribution.

\section{Tests of Multiple Parameters}

Similar tests of the sums $A, B, \Gamma$ and $\Delta$, in equations (2.6) and (2.7), may be conducted using the methods described above. Using $\alpha$ to denote the vector of parameters $\alpha_{i}(i=1, \ldots, 5)$, the variances of the sums of parameters $\alpha_{i}\left(\Sigma_{\hat{\alpha} \hat{\alpha}}\right)$ or the other sums can be computed directly from the estimated covariance matrix $\Sigma_{\hat{\Theta}}$. 


\section{Tests of the Differences in Parameters}

The setup for testing the difference in $\alpha$ before and after the event is as follows. The null hypothesis is $H_{0}: \lambda=\alpha_{\text {After }}-\alpha_{\text {Before }}=0$ against the two-sided alternative $H_{1}: \lambda \neq 0$. Under the standard assumption

$$
\hat{\lambda}=\hat{\alpha}_{\text {After }}-\hat{\alpha}_{\text {Before }} \sim N\left(\lambda, \sigma_{\text {After }}^{2}+\sigma_{\text {Before }}^{2}\right),
$$

suggests a conventional $t$-test for the difference between two means. However, the standard test is not robust to differences in variance before and after. A better test follows Scott \& Smith (1971) as above and is

$$
Z=\left(\hat{\alpha}_{\text {After }}-\hat{\alpha}_{\text {Before }}\right) / \sqrt{\hat{\sigma}_{\text {After }}^{2}+\hat{\sigma}_{\text {Before }}^{2}},
$$

which has a standard normal distribution. Tests of the sum of the $\lambda_{i}$ over the $n$ stock/events may be performed using the same procedures in the preceding sections, noting that the estimated variance of $\hat{\lambda}$ is computed by $\hat{\sigma}_{\text {After }}^{2}+\hat{\sigma}_{\text {Before }}^{2}$. For further technical details about equations (2.9) and (2.10), see the discussion at the end of Section 2.3.1.

\subsection{Data Description}

This section describes the data used for this chapter as well as the procedures for data cleaning. The study in this chapter covers a period of 10 years from May 2001 to May 2011. During this period, the D-list for short selling has been revised 86 times including 41 quarterly announcements and 45 single additions for IPO firms, with a total of 1,296 addition events and 810 deletion events. An addition (deletion) event is defined when an individual stocks is added to (removed from) 
the D-list for short selling from the effective date. When an individual stock reenters (re-quits) the D-list, it is regarded as another addition (deletion) event. The changes are summarised in Table 2.1 including the records of additions and deletions from the D-list. In May 2011 there were 760 stocks on the D-list.

[Insert Table 2.1 about here]

The unit trust, exchange traded funds, mutual funds, investment companies and stocks traded on the Growth Enterprise Market (GEM) are excluded from the analysis. Addition events for IPO firms are excluded due to the inadequacy of observations in the pre-event period. Stocks with options and futures written are excluded as option and future trading can be considered as an alternative to short selling but at a lower cost (Diamond \& Verrecchia, 1987). The sample selection procedure above reduces the final data set to 1,127 addition events and 728 deletion events.

The transaction data and bid and ask prices are obtained from Trade Record and Bid and Ask Record from the HKEx, respectively. The Bid and Ask Record provides the best five bid and ask prices and quantities in the limit order book. Bid and Ask Record provides only changes which means that a data value is available only if it is different from the one previously provided. Prior to January 2008, the information is recorded at 30-second intervals with timestamp accuracy of one second $^{10}$. Since 2008, it has been recorded for every change with timestamp accuracy of 0.001 second. The Trade Record provides transaction prices and volumes with a time stamp to the nearest second.

All the trades occurring outside the normal opening hours are excluded. This covers the period before 9:00 a.m. and after 4:00 p.m. and those during the pre-opening

\footnotetext{
${ }^{10}$ This chapter assumes that bid and ask information does not change within the 30 second intervals.
} 
session (between 9:00 a.m. and 9:30 a.m.) as the trading mechanism in the preopening session is different from the regular trading session ${ }^{11}$. To avoid overnight news arrival, the first quote return of each day is treated as a missing value for all lagged variables. Trades with the same time stamp and the same price are treated as one trade by adding up the volume corresponding to individual trades. For trades with the same time stamp but the different price, $(i)$ calculate the signed volume weighted average price and the absolute value of the sum of signed volume for multiple trades, (ii) assign a new trade sign to the aggregated volume by the classification of Lee \& Ready (1991). Anomalous data caused by systematic errors, such as zero bid and ask price and negative spreads are discarded. The estimation of the model for each stock for each event is based on all transactions in the 60-day period before and after the event date. All univariate regressions are estimated for each equation separately with the White heteroscedasticity correction for standard errors (White, 1980). The bona fide VAR model is estimated as a whole system using the maximum likelihood method with consideration of contemporaneous correlation between residuals in two equations. All model estimations are on an unweighted and weighted basis and are denoted as OLS, WLS, VAR and WVAR respectively.

Combining and processing the two datasets: Trade Record and Bid and Ask Record is a complex and time-consuming task due to the huge size of data (nearly 95GB for Trade Record and 1.5TB for Bid and Ask Record) on the high frequency basis over a 10-year period. Thus, this study uses powerful database software, such as SQL, to process the records. First, trade price and quantity as well as the current bid and ask prices are extracted from the datasets, respectively. Secondly, the transaction data and bid and ask prices are merged together and then sorted by time by using SQL. The nearest mid-quotes before and after each transaction are determined as the prevailing and the subsequent mid-quotes of a trade by using Perl. The prevailing mid-quotes are used to define the trade sign under the classification of Lee \& Ready (1991). The prevailing and the subsequent mid-quotes are used to

\footnotetext{
${ }^{11}$ Detailed information about the trading mechanism in the pre-opening and regular trading sessions can be found at http://www.hkex.com.hk/eng/market/sec_tradinfra/tradmech.htm.
} 
calculate the quote returns. The resulting database is nearly $2 \mathrm{~GB}$ in size. An example of the data format is shown in Appendix D.

\section{$2.5 \quad$ Results}

The results described in this section use 60-day transaction data (before and after the event) for model estimation and are based on 1,127 addition events and 728 deletion events. As the D-list for short sales is reviewed by the HKEx on the quarterly basis to add or remove stocks from the list, it means that 60 -day (3 months) period is the appropriate period for testing the effect of short sales in the speed of price adjustment during each individual event. Moreover, a 60-day period gives a sufficient estimation window for comparison before and after an event with consideration of the availability of lending shares for short sales. Previous studies also suggest that the 60-day trading window is sufficient to allow reasonably precise estimation of the parameters (Easley \& O'Hara, 1992; Easley et al., 1997). The SS test is used throughout in place of the standard $t$-test and the significance level is 1\%. All tests of significance in the thesis are at the 1\% level. First, Section 2.5.1 provides a statistical summary for the stocks in the data set during the 10-year period. Section 2.5.2 reports validation tests under the OLS, WLS, VAR, and WVAR models using the procedure described in Section 2.3.2. Section 2.5.3 presents the results about changes in model parameters as a result of addition/deletion event. Sections 2.5.4 demonstrates the results for dynamics and p-values of model parameters for both addition/deletion events. Sections 2.5.5 reports the $Z$ scores for tests on difference in model parameters for both events. Sections 2.5.6 reports results on model selection. From Section 2.5.3 onwards, detailed results are presented for OLS and summarised for the other three models as the findings of the other three models are qualitatively similar to those by OLS. A full set of detailed results for the WLS, VAR and WVAR models is available in Appendix C. 


\subsubsection{Statistics Summary}

Table 2.2 presents a summary statistics for all sample stocks for the period 20012011.

[Insert Table 2.2 about here]

As shown in Table 2.2, the number of trades for addition stocks decreases from 10,654 to 8,650 after they become shortable, while the number of trades for deletion stocks trade stay at similar level after they are taken from the D-list. The number of trades for addition stocks in both pre- and post- event window are much higher than those for deletion stocks. It suggests that addition stocks are traded more actively during the 60-day period. Stocks for the addition events are traded generally at a higher average price than those for the deletion events in both the before and after periods (3.77 vs. 2.26 and 3.85 vs. 2.29, respectively). Quote returns for both addition and deletion stocks stay similar to the average level. The average trade durations for addition stocks in the before and after event are 369 and 409 seconds, while for deletion stocks are 705 and 762 seconds respectively. This is consistent with the fact that the trading for addition stocks is more frequent. The average aggregated 60-day trading volume for addition stocks is more than that for deletion stocks in the before and after periods (1,088,996 vs. 410,697 and 631,793 vs. 478,416, respectively). It shows that stocks for the addition events have higher level of interest and liquidity in the market than those for the deletion events. The signed volume indicates the average aggregated 60-day trading volume with trade sign. The signed volume decreases for addition stocks and increases for deletion stocks. This implies that more trades initiated by a seller are triggered when shocks are added to the list for short sales and there are fewer trades from sellers when stocks are removed from the list. 


\subsubsection{Model Validation}

The bivariate VAR model used in this chapter is modified by adding a constant in each equation. A consistency check of the parameter in the modified model is conducted using the procedure described in Section 2.3.2. The $Z$ statistic defined at (2.3) in Section 2.3.2 has a standard normal distribution under the null hypothesis . Rejection of the null hypothesis could indicate inconsistency of the model specification at (2.1). Table 2.3 contains the results for the OLS, WLS, VAR and WVAR models. The results show that for both addition and deletion events, the models are validated according to the test for almost all stocks. Compared with the results for OLS and WLS, all stocks are estimated using VAR and WVAR with a validated model. The overall $Z$ scores, shown in the last column of Table 2.3 are computed for all sample stocks. As the table shows, the $Z$ scores for all four methods are very small thus supporting the view that the model at (2.1) is well specified for this data set.

[Insert Table 2.3 about here]

\subsubsection{Changes in Model Parameters}

To examine whether short selling improves price efficiency, the estimated parameters from the models are compared before and after the addition/deletion events. For the OLS model, Table 2.4 shows the number of parameter estimates for the addition events which are significantly less than zero, significantly greater than zero or which fail to achieve statistical significance. The table has four vertical sections and two horizontal panels. The first panel shows results for individual parameters. The second panel shows results for the sums of the $\alpha \mathrm{s}, \beta_{\mathrm{s}}, \gamma_{\mathrm{s}}$ and $\delta$ s respectively. The first vertical section show the numbers of stocks for which the corresponding parameter is significantly less than zero, neutral or significantly greater than zero before the addition event. The second vertical section shows the corresponding results after the addition event. The section headed "Chsq" shows the value of a 
Chi-squared statistic that is used to test the difference between the observed frequencies in the before and the after period for each variable.

The results in the Chi-squared score column, show that none of the observed sets of frequencies for each parameter exhibit significant differences. The last section presents number of differences in parameters during the addition events. It shows the numbers of stocks for which the corresponding parameter exhibits significant decrease, neutral change or significant increase in its magnitude during the addition events. Differences are calculated by absolute value of model parameters between after and before the addition event. Cells in which there is a substantial number of statistically significant changes are shown in bold. Using 112 (or $10 \%$ of the total number of 1,127 addition events) as a threshold shows that there is a substantial number of changes in $\alpha_{1}$ (the first lagged quote autocorrelation), $\beta_{0}$ (the price impact of the contemporaneous trade), $\beta_{1}$ and the sum $B$. Overall, the aggregated price impact of trades is affected by short selling for nearly half of addition stocks, among which there is generally a stronger price impact of trades. However, there is only a small number of addition stocks experiencing significant change in quote autocorrelations and trade continuity during the events.

[Insert Tables 2.4 and 2.5 about here]

The corresponding results for the deletion events are reported in Table 2.5. The results show that the majority of individual parameters do not change significantly. Using 72 stocks (10\% of the total number of 728 deletion events) as a threshold indicates that there is a significant number of changes for $\alpha_{1}$, the contemporaneous parameter $\beta_{0}$ and the sum $B$. The results show that for these stocks, there is generally a greater quote autocorrelation and a weaker price impact of trades when they are deleted from the short selling list.

Similar results based on the WLS model are omitted here, but are available in Appendix C. Briefly, WLS reduces the number of estimated parameters which are significantly different from zero and, as a consequence, the number of significant 
differences during both addition and deletion events. Qualitatively, the findings are similar to those reported for the OLS model.

The corresponding results for the VAR model are shown in Tables 2.6 and 2.7. In Table 2.6, for the addition events, there are significant changes in the parameters $\alpha_{1}, \beta_{0}, \beta_{1}$ and the sum $B$, as is the case for the OLS model. In addition, there is a significant number of changes in $\alpha_{2}$ (quote revision autocorrelations), $\beta_{2}$ (price impact of trades), $\delta_{1}$ (trade continuity), the sum $A$ and the sum $\Delta$. The results indicate that the effect of short sales during the addition events spreads to more parameters when the model at (2.1) is estimated simultaneously. Table 2.7 shows the corresponding results for the deletion events under the VAR model. There are more significant changes under the VAR model than the case for the deletion events under OLS, but fewer than for the addition events. The corresponding results for the WVAR model are in Appendix C.

[Insert Tables 2.6 and 2.7 about here]

So far, the results for changes due to both addition and deletion events show that only some model parameters are affected and that the changes in quote autocorrelation and trade continuity apply to a minority of stocks. For the stocks that are significantly affected, the parameters that change as a result of an addition or deletion event are those that measure quote revision autocorrelations, price impact of trades and trade autocorrelations. However, the overall effect of short selling is not clear as significant changes can be positive or negative. Table 2.8 contains a summary of the differences between the four models for the addition events. The table is a summary of more detailed results, which are available in Appendix C. The table has four panels. In panel $(i)$, the results from the WLS model are compared with those from OLS. The titles of panels $(i i)$ to $(i v)$ indicate the other model comparisons. The table has three vertical sections: parameters before the addition event, after it and the differences. The rows of the table corresponding to those parameters for which the number of significant differences (either significant increase or decrease in the magnitude of parameters) is at least 112, that is about 
$10 \%$ of the 1,127 addition events. The interpretation of the table entries is best illustrated by an example.

In Table 2.4, the OLS model for the addition events, there are 1,055 stock/events for which the estimates of the parameter $\delta_{1}$ do not change significantly. For the corresponding entry for the VAR model in Table 2.6, there are 929 such events. Thus, in panel ( iii) of Table 2.8 the entry in the corresponding cell is $-126=929-1,055$; that is, 126 less stock/events exhibit no change in the estimated value of $\delta_{1}$ under VAR than under OLS. There is a corresponding increase in the number of negative and positive changes. Panel (iii) shows that, where the number of changes is greater than (or very close to) the threshold, the corresponding cells for other parameters are also negative. This then suggests that that the OLS model is more conservative than VAR; the number of estimated parameters and changes to parameters which are significantly different from zero is increased.

[Insert Table 2.8 about here]

In panels (iii) and (iv) of Table 2.8, the entries in the corresponding cells are negative, thus suggesting that both the VAR and WVAR models are more sensitive than their least squares counterparts; the number of estimated parameters and changes to parameters which are significantly different from zero increases compared to the results for OLS and WLS. The results in panel $(i)$ suggest that the WLS model is more conservative than OLS; the results in panel (ii) are mixed.

[Insert Table 2.9 about here]

Table 2.9 shows the corresponding results for the deletion events. Table 2.9 has the same rows as Table 2.8 to facilitate comparison, but the threshold is 72 events. The third vertical section of the table shows that the number of changes in the estimated parameters is lower for the deletion events. Where there are a significant number of changes, however, the two VAR models are, as above, more sensitive 
than their least squares counterparts. WLS is somewhat more conservative than OLS and the results are once again mixed for panel $(i i)$.

The difference comparison results summarised above for both events show that less stocks exhibit significant changes especially in the price impact of trades when inter-trade time durations are used while in general there are more stocks with significant changes in the autocorrelations in quote returns and trades if the two model equations are estimated simultaneously.

\subsubsection{Parameter Dynamics}

A study of parameter dynamics is carried out to check the consistency in the sign of model parameters before and after addition and deletion events. Table 2.10 shows parameter dynamics for both addition and deletion events for the OLS model. Tables for individual model parameters are available in Appendix C. The table has four panels which show the results summed over all model parameters $A, B, \Gamma$ and $\Delta$. The horizontal and vertical directions of each significance category denote before and after the event, respectively. The table is divided vertically into two sections for addition and deletion events. The predominant cells are shown in bold. In the first row of the addition section, $83.85 \%$ of stock/events have an estimated value of parameter $A$ (quote revision autocorrelations) which is less than zero at the $1 \%$ level of significance both before and after the addition event. In the deletion section, the last row of the table $3.57 \%$ of sample stocks have an estimated value of parameter $\Delta$ (trade continuity) which is positive and significant at the $1 \%$ level before the deletion event and is positive and significant at the $5 \%$ level after the event. These results show that the majority of the sums of estimated model parameters, with the exception of the sum $\Gamma$, have consistent signs both before and after addition and deletion events. Thus, even for stocks that exhibit significant changes in individual estimated parameter values, the general performance of returns according to the OLS model remains the same in most cases. 
[Insert Table 2.10 about here]

The corresponding results for WLS, VAR and WVAR are omitted, but a summary is provided in Table 2.11 with details in Appendix C. As noted in Table 2.10, the dominant cells are all in the diagonals of each sub-table, indicating consistent sign before and after the event. Table 2.11 reports the corresponding percentages for all four models. The summary broadly confirms the results in this section. It is interesting to note that there is less parameter consistency when weighted models are used.

[Insert Table 2.11 about here]

\subsection{5 $Z$ Scores for Tests of Difference in Model Parame- ters}

To examine the effect of short selling, Table 2.12 summarises the $Z$ scores for tests of the differences in estimated model parameters before and after addition and deletion events for the OLS, WLS, VAR and WVAR models. The $Z$ scores for the differences are computed using the SS test statistic at (2.10). For both addition and deletion sections, there are four sub-sections corresponding to the test results for the four models. In this table, $Z$ scores shown in bold are significantly different from zero at the $1 \%$ level.

[Insert Table 2.12 about here]

For the addition events, the parameter $\beta_{0}$ which captures the contemporaneous price impact of trades increases in value at the $1 \%$ significance level when the model is estimated by WLS. It therefore indicates that, when time duration is considered, on average the quote midpoint rises (falls) more immediately subsequent 
to a purchase (sell) order if stocks are shortable. For the lagged variables $\beta_{1}, \ldots, \beta_{5}$, few significant changes are observed under any of the four models. The change in the estimated aggregated price impact parameter $B$ is also significantly greater than zero under the WLS and WVAR models. It implies that short sales strengthen the price impact of trades with main contribution from current trades under the consideration of trade duration. For quote revision, it is found that the sum $A$ which measures the aggregated autocorrelation in quote revision significantly decreases during the addition events under WVAR. The sum $B$ measuring the price impact of trades increases after stocks being added to the D-list by using WLS and WVAR. There are statistically significant changes in the aggregate values of $\Gamma$ (Granger causality) for four models however the changes are not consistent as those are positive for OLS and VAR and negative if the estimations are weighted. The aggregated trade continuity, the sum $\Delta$, decreases and the significance level is at the $1 \%$ significance for all models. The overall results for the addition events show that the trades become less correlated and this pattern keeps the same under four estimation models. The quote autocorrelation also decreases when it is estimated by WVAR. The price impact of trades has been enhanced by short sales if the time duration is considered. The effect of short sales on Granger causality is not conclusive.

For the deletion events, less parameters experience significant changes. For quote revision, it is found that the estimated values of the sum $A$ including several corresponding lagged parameters $\alpha$ increase at the $1 \%$ significance level by OLS. There are no significant changes for lagged parameters $\alpha$ for other three models. Overall, the aggregated quote autocorrelations become stronger if stocks are removed from the short-selling list in three out of four models. The aggregated trade continuity $\Delta$ is not observed to have a significant change in value under four models, thus indicating that trade continuity is not affected significantly during the deletion events. Similar as the addition events, there is a significant decrease in the aggregated Granger causality when there is consideration of inter-trade times. By contrast, there are significant increases when OLS and VAR are used. Therefore, the effect of short sales on Granger causality remains unclear. Different from the addition events, the changes in price impact of trades stay neutral which implies that short 
sales do not affect price impact of trades during the deletion events.

In sum, it is found that the decrease in trade continuity is the most consistent change during the addition events while the increase in quote autocorrelations stands out from the crowd during the deletion events. When stocks become shortable, trades continuity experiences less autocorrelations. When stocks lose their eligibility of short sales, quote autocorrelations become stronger. The price impact of trades gets enhanced by short sales during the addition events when time duration is counted. Lower autocorrelations in both trade continuity and quote revision for addition stocks and greater autocorrelations in quote revision for deletion stocks suggest that the speed of price adjustment is accelerated by short sales.

\subsubsection{Model Selection}

This section provides some indications of the best model based on the correlation test of residuals and the summary of model estimations.

Formal model selection between OLS and VAR models can be made using a likelihood ratio test which is asymptotically equivalent to a test of the correlation between the two time series of residuals from the VAR model. Table 2.13 reports the residual correlation tests under four models. The first vertical section presents the number of stock/events with no less than 100 transactions before and after each addition and deletion event. Each cell in the second vertical section shows the percentage of stock/events for which the residual correlation is significant at $1 \%$. The results show that no residual correlations is observed under OLS and WLS while there is a significant residual correlation for the majority of stocks under the VAR and WVAR models. It indicates that the ability to capture residual correlations between quote and trade equations is hugely better under the VAR and WVAR models.

[Insert Table 2.13 about here] 
Table 2.14 provides a summary of model estimations for addition and deletion events. The significant changes are counted only if the autocorrelations of parameters measuring the speed of price adjustment decrease (increase) during the addition (deletion) events. The table shows the percentage of changes at the specified level of significance under each model. For instance, for the addition events, under the VAR model, $22.63 \%$ of stocks/events experience a faster speed of price adjustment at the $1 \%$ significance level. The figures in the table indicate that the VAR and WVAR models have better abilities to capture the changes during both events.

[Insert Table 2.14 about here]

A test of WLS vs. OLS or WVAR vs. VAR is non-standard and there is no direct test to the best of our knowledge for comparison between models with and without consideration of time duration. According to the evidences shown above, the WVAR model is considered as the preferred model as it has a better ability to capture the effect of short sales and the weighted method is more theoretically sound.

\subsection{Conclusions}

The institutional environment in Hong Kong offers a unique setting to investigate the effect of short sales constraints. By using the intraday data for a 10-year period, this chapter investigates the speed of price adjustment for stocks before and after the change of their eligibility of short selling. This work uses a bivariate VAR model based on four different estimation methods, namely OLS, WLS, VAR and WVAR. From a technical perspective, examining the averages of individual parameters requires non-standard approaches. This is because, even under the assumptions of the model, the estimated values of a given parameter do not have constant variance. Therefore, in addition to some standard tests commonly carried 
out for regression analysis, this chapter also applies some non-standard statistical tests to facilitate a more robust investigation of the estimation results.

The main conclusions of this chapter are as follows. There is a subset of stocks for which certain parameters in the model do exhibit significant changes which implies that eligibility for short selling or loss thereof does aid price discovery, as shown by tests carried out for individual stocks before and after addition/deletion events. The parameters which measure trade continuity (quote autocorrelation) are the most affected during the addition (deletion) events. The decreased (increased) autocorrelations during the addition (deletion) events indicates that more (less) quote midpoints are likely to follow a random walk. The results are consistent with those from Boehmer \& Wu (2013). It is also observed that trade continuity becomes stronger when stocks are removed from the D-list. Both significant changes in quote revision and trade continuity indicates greater price discovery by short sales. The study of parameter dynamics indicates that model parameters largely remain consistent. For example a negative parameter that is statistically significant will generally remain in the same category, even if the estimated value changes as a result of the addition/deletion event.

For model selection, it is found that VAR and WVAR models are better in capturing the residual correlations and the effect of short sales rather than OLS and WLS during both events. The WVAR model is considered as the preferred model to investigate price efficiency with short sales as the time-weighted model is more theoretically sound.

High frequency returns invariably exhibit heterogeneity of variance. Even though the return variables in this chapter have a non-standard definition, it is conjectured that heterogeneity of variance may have an effect on other model parameters and, if so, on the subsequent inferences. The next step in research in this area is to employ GARCH models. 


\section{Table 2.1: The Records of Addition and Deletion Events of the D-list on the HKEx}

The table reports the revision history of short selling list on the HKEx during the study period of 20012011. Columns are as follows: 1. Announcement date; 2. Effective date; 3. No. of additions; 4. No. of deletions; 5 . No. of stocks on the list after each revision.

\begin{tabular}{cccccccccc}
\hline $\mathbf{1}$ & $\mathbf{2}$ & $\mathbf{3}$ & $\mathbf{4}$ & $\mathbf{5}$ & $\mathbf{1}$ & $\mathbf{2}$ & $\mathbf{3}$ & $\mathbf{4}$ & $\mathbf{5}$ \\
\hline $03 / 05 / 2001$ & $14 / 05 / 2001$ & 6 & 0 & 280 & $14 / 05 / 2007$ & $21 / 05 / 2007$ & 29 & 14 & 451 \\
$07 / 08 / 2001$ & $20 / 08 / 2001$ & 9 & 11 & 278 & $18 / 05 / 2007$ & $21 / 05 / 2007$ & 1 & 0 & 452 \\
$27 / 11 / 2001$ & $03 / 12 / 2001$ & 17 & 85 & 210 & $22 / 05 / 2007$ & $29 / 05 / 2007$ & 1 & 0 & 453 \\
$05 / 02 / 2002$ & $25 / 02 / 2002$ & 7 & 14 & 203 & $27 / 06 / 2007$ & $04 / 07 / 2007$ & 1 & 0 & 454 \\
$08 / 05 / 2002$ & $21 / 05 / 2002$ & 11 & 6 & 208 & $16 / 07 / 2007$ & $17 / 07 / 2007$ & 1 & 0 & 455 \\
$17 / 07 / 2002$ & $29 / 07 / 2002$ & 24 & 5 & 227 & $06 / 08 / 2007$ & $13 / 08 / 2007$ & 134 & 9 & 580 \\
$28 / 11 / 2002$ & $29 / 11 / 2002$ & 6 & 15 & 218 & $20 / 08 / 2007$ & $27 / 08 / 2007$ & 1 & 0 & 581 \\
$21 / 01 / 2003$ & $27 / 01 / 2003$ & 5 & 7 & 216 & $19 / 11 / 2007$ & $26 / 11 / 2007$ & 64 & 23 & 622 \\
$07 / 05 / 2003$ & $19 / 05 / 2003$ & 18 & 7 & 227 & $07 / 12 / 2007$ & $14 / 12 / 2007$ & 2 & 0 & 624 \\
$14 / 07 / 2003$ & $21 / 07 / 2003$ & 1 & 16 & 212 & $11 / 12 / 2007$ & $14 / 12 / 2007$ & 1 & 0 & 625 \\
$27 / 10 / 2003$ & $03 / 11 / 2003$ & 36 & 5 & 243 & $11 / 02 / 2008$ & $18 / 02 / 2008$ & 33 & 41 & 617 \\
$05 / 01 / 2004$ & $06 / 01 / 2004$ & 1 & 0 & 244 & $11 / 03 / 2008$ & $13 / 03 / 2008$ & 1 & 0 & 618 \\
$03 / 02 / 2004$ & $10 / 02 / 2004$ & 29 & 3 & 270 & $05 / 05 / 2008$ & $13 / 05 / 2008$ & 22 & 47 & 593 \\
$06 / 04 / 2004$ & $07 / 04 / 2004$ & 1 & 0 & 271 & $14 / 05 / 2008$ & $15 / 05 / 2008$ & 1 & 0 & 594 \\
$20 / 04 / 2004$ & $27 / 04 / 2004$ & 26 & 4 & 293 & $02 / 06 / 2008$ & $03 / 06 / 2008$ & 5 & 0 & 599 \\
$25 / 06 / 2004$ & $01 / 07 / 2004$ & 1 & 0 & 294 & $31 / 07 / 2008$ & $07 / 08 / 2008$ & 10 & 51 & 558 \\
$05 / 07 / 2004$ & $09 / 07 / 2004$ & 1 & 0 & 295 & $07 / 11 / 2008$ & $14 / 11 / 2008$ & 6 & 144 & 420 \\
$26 / 07 / 2004$ & $02 / 08 / 2004$ & 8 & 21 & 282 & $05 / 02 / 2009$ & $12 / 02 / 2009$ & 25 & 27 & 418 \\
$01 / 11 / 2004$ & $08 / 11 / 2004$ & 9 & 11 & 280 & $07 / 05 / 2009$ & $14 / 05 / 2009$ & 13 & 22 & 409 \\
$28 / 01 / 2005$ & $07 / 02 / 2005$ & 15 & 7 & 288 & $03 / 07 / 2009$ & $10 / 07 / 2009$ & 1 & 0 & 410 \\
$28 / 02 / 2005$ & $01 / 03 / 2005$ & 2 & 0 & 290 & $29 / 07 / 2009$ & $05 / 08 / 2009$ & 49 & 16 & 443 \\
$09 / 05 / 2005$ & $17 / 05 / 2005$ & 37 & 9 & 318 & $29 / 10 / 2009$ & $05 / 11 / 2009$ & 58 & 11 & 490 \\
\hline
\end{tabular}

Table continued on the following page. 
Table 2.1: Continued from previous page

\begin{tabular}{cccccccccc}
\hline $\mathbf{1}$ & $\mathbf{2}$ & $\mathbf{3}$ & $\mathbf{4}$ & $\mathbf{5}$ & $\mathbf{1}$ & $\mathbf{2}$ & $\mathbf{3}$ & $\mathbf{4}$ & $\mathbf{5}$ \\
\hline $04 / 07 / 2005$ & $08 / 07 / 2005$ & 1 & 0 & 319 & $11 / 11 / 2009$ & $18 / 11 / 2009$ & 1 & 0 & 491 \\
$11 / 07 / 2005$ & $15 / 07 / 2005$ & 1 & 0 & 320 & $30 / 11 / 2009$ & $03 / 12 / 2009$ & 1 & 0 & 492 \\
$04 / 08 / 2005$ & $15 / 08 / 2005$ & 14 & 12 & 322 & $08 / 12 / 2009$ & $15 / 12 / 2009$ & 1 & 0 & 493 \\
$26 / 08 / 2005$ & $05 / 09 / 2005$ & 1 & 0 & 323 & $21 / 12 / 2009$ & $24 / 12 / 2009$ & 1 & 0 & 494 \\
$24 / 10 / 2005$ & $28 / 10 / 2005$ & 1 & 0 & 324 & $25 / 01 / 2010$ & $01 / 02 / 2010$ & 65 & 8 & 551 \\
$18 / 11 / 2005$ & $17 / 11 / 2005$ & 11 & 7 & 328 & $22 / 02 / 2010$ & $01 / 03 / 2010$ & 1 & 0 & 552 \\
$14 / 02 / 2006$ & $20 / 02 / 2006$ & 10 & 8 & 330 & $03 / 03 / 2010$ & $10 / 03 / 2010$ & 1 & 0 & 553 \\
$27 / 02 / 2006$ & $01 / 03 / 2006$ & 2 & 0 & 332 & $18 / 03 / 2010$ & $25 / 03 / 2010$ & 1 & 0 & 554 \\
$19 / 05 / 2006$ & $29 / 05 / 2006$ & 23 & 17 & 338 & $03 / 05 / 2010$ & $10 / 05 / 2010$ & 59 & 12 & 601 \\
$29 / 05 / 2006$ & $02 / 06 / 2006$ & 1 & 0 & 339 & $12 / 07 / 2010$ & $16 / 07 / 2010$ & 1 & 0 & 602 \\
$01 / 06 / 2006$ & $02 / 06 / 2006$ & 1 & 0 & 340 & $28 / 07 / 2010$ & $04 / 08 / 2010$ & 40 & 19 & 623 \\
$18 / 08 / 2006$ & $25 / 08 / 2006$ & 38 & 10 & 368 & $23 / 08 / 2010$ & $30 / 08 / 2010$ & 1 & 0 & 624 \\
$24 / 08 / 2006$ & $01 / 09 / 2006$ & 1 & 0 & 369 & $25 / 10 / 2010$ & $29 / 10 / 2010$ & 47 & 18 & 653 \\
$19 / 10 / 2006$ & $23 / 10 / 2006$ & 1 & 0 & 370 & $11 / 11 / 2010$ & $15 / 11 / 2010$ & 1 & 0 & 654 \\
$19 / 10 / 2006$ & $27 / 10 / 2006$ & 1 & 0 & 371 & $17 / 11 / 2010$ & $22 / 11 / 2010$ & 2 & 0 & 656 \\
$24 / 11 / 2006$ & $01 / 12 / 2006$ & 55 & 9 & 417 & $17 / 12 / 2010$ & $20 / 12 / 2010$ & 1 & 0 & 657 \\
$26 / 02 / 2007$ & $05 / 03 / 2007$ & 30 & 24 & 423 & $23 / 12 / 2010$ & $30 / 12 / 2010$ & 1 & 0 & 658 \\
$13 / 03 / 2007$ & $14 / 03 / 2007$ & 1 & 0 & 424 & $12 / 01 / 2011$ & $28 / 01 / 2011$ & 1 & 0 & 659 \\
$12 / 04 / 2007$ & $19 / 04 / 2007$ & 5 & 0 & 429 & $25 / 01 / 2011$ & $01 / 02 / 2011$ & 1 & 0 & 660 \\
$25 / 04 / 2007$ & $26 / 04 / 2007$ & 6 & 0 & 435 & $18 / 02 / 2011$ & $25 / 02 / 2011$ & 70 & 17 & 713 \\
$25 / 04 / 2007$ & $27 / 04 / 2007$ & 1 & 0 & 436 & $17 / 05 / 2011$ & $24 / 05 / 2011$ & 65 & 18 & 760 \\
\hline
\end{tabular}




\section{Table 2.2: Summary Statistics}

This table describes the data including trades and quotes for 1,127 addition stocks and 728 deletion stocks in a 60-day period before and after the event. Midquote is the average of bid and ask quotes after the trade. Quote Return is the log midquote change. Trade Duration is measured in seconds. Volume is the sum of the volume per transaction in a 60-day period. Signed Volume is the sum of the volume with its trade sign in a 60-day period ( 1 for a buy; -1 for a sale; and 0 otherwise). Each variable is calculated for each stock for each event in a 60-day period and compute the average across the stocks.

\begin{tabular}{|c|c|c|c|c|}
\hline & \multicolumn{2}{|c|}{ Addition } & \multicolumn{2}{|c|}{ Deletion } \\
\hline \multirow[t]{2}{*}{ No. of Stocks } & \multicolumn{2}{|c|}{1,127} & \multicolumn{2}{|c|}{728} \\
\hline & Before & After & Before & After \\
\hline No. of Trades & 10,654 & 8,650 & 3,495 & 3,722 \\
\hline \multicolumn{5}{|c|}{ Trade Price } \\
\hline Mean & 3.77 & 3.85 & 2.26 & 2.29 \\
\hline Max & 4.47 & 4.55 & 2.74 & 2.70 \\
\hline Min & 3.09 & 3.16 & 1.84 & 1.92 \\
\hline Std. Dev. & 0.78 & 0.76 & 0.57 & 0.62 \\
\hline \multicolumn{5}{|c|}{ Midquote } \\
\hline Mean & 3.77 & 3.85 & 2.26 & 2.29 \\
\hline Max & 4.47 & 4.55 & 2.77 & 2.72 \\
\hline Min & 3.09 & 3.15 & 1.85 & 1.91 \\
\hline Std. Dev. & 0.78 & 0.76 & 0.57 & 0.63 \\
\hline \multicolumn{5}{|c|}{ Quote Return } \\
\hline Mean & $0.000 \%$ & $-0.001 \%$ & $-0.003 \%$ & $-0.004 \%$ \\
\hline Max & $5.099 \%$ & $5.388 \%$ & $10.656 \%$ & $7.702 \%$ \\
\hline Min & $-5.164 \%$ & $-5.525 \%$ & $-11.294 \%$ & $-8.530 \%$ \\
\hline Std. Dev. & $0.610 \%$ & $0.661 \%$ & $1.498 \%$ & $1.467 \%$ \\
\hline \multicolumn{5}{|c|}{ Trade Duration } \\
\hline Mean & 369 & 409 & 705 & 762 \\
\hline $\operatorname{Max}$ & 10,890 & 11,319 & 13,395 & 13,539 \\
\hline Min & 1 & 1 & 1 & 1 \\
\hline Std. Dev. & 1,298 & 1,389 & 1,945 & 2,019 \\
\hline
\end{tabular}

Table continued on the following page. 
Table 2.2: Continued from previous page

\begin{tabular}{|c|c|c|c|c|}
\hline & \multicolumn{2}{|c|}{ Addition } & \multicolumn{2}{|c|}{ Deletion } \\
\hline & Before & After & Before & After \\
\hline \multicolumn{5}{|c|}{ Volume (1,000 Shares) } \\
\hline Mean & $1,088,996$ & 631,793 & 410,697 & 478,416 \\
\hline Max & $183,732,080$ & $42,180,880$ & $26,394,260$ & $53,770,050$ \\
\hline Min & 67 & 55 & 404 & 256 \\
\hline Std. Dev. & $6,192,789$ & $1,900,829$ & $1,403,265$ & $2,428,428$ \\
\hline \multicolumn{5}{|c|}{ Signed Volume (1,000 Shares) } \\
\hline Mean & 46,686 & 23,381 & 6,056 & 27,330 \\
\hline Max & $9,738,720$ & $2,357,200$ & $7,694,430$ & $8,728,600$ \\
\hline Min & $-1,528,908$ & $-1,624,540$ & $-1,943,920$ & $-784,525$ \\
\hline Std. Dev. & 377,875 & 166,297 & 322,513 & 458,057 \\
\hline
\end{tabular}




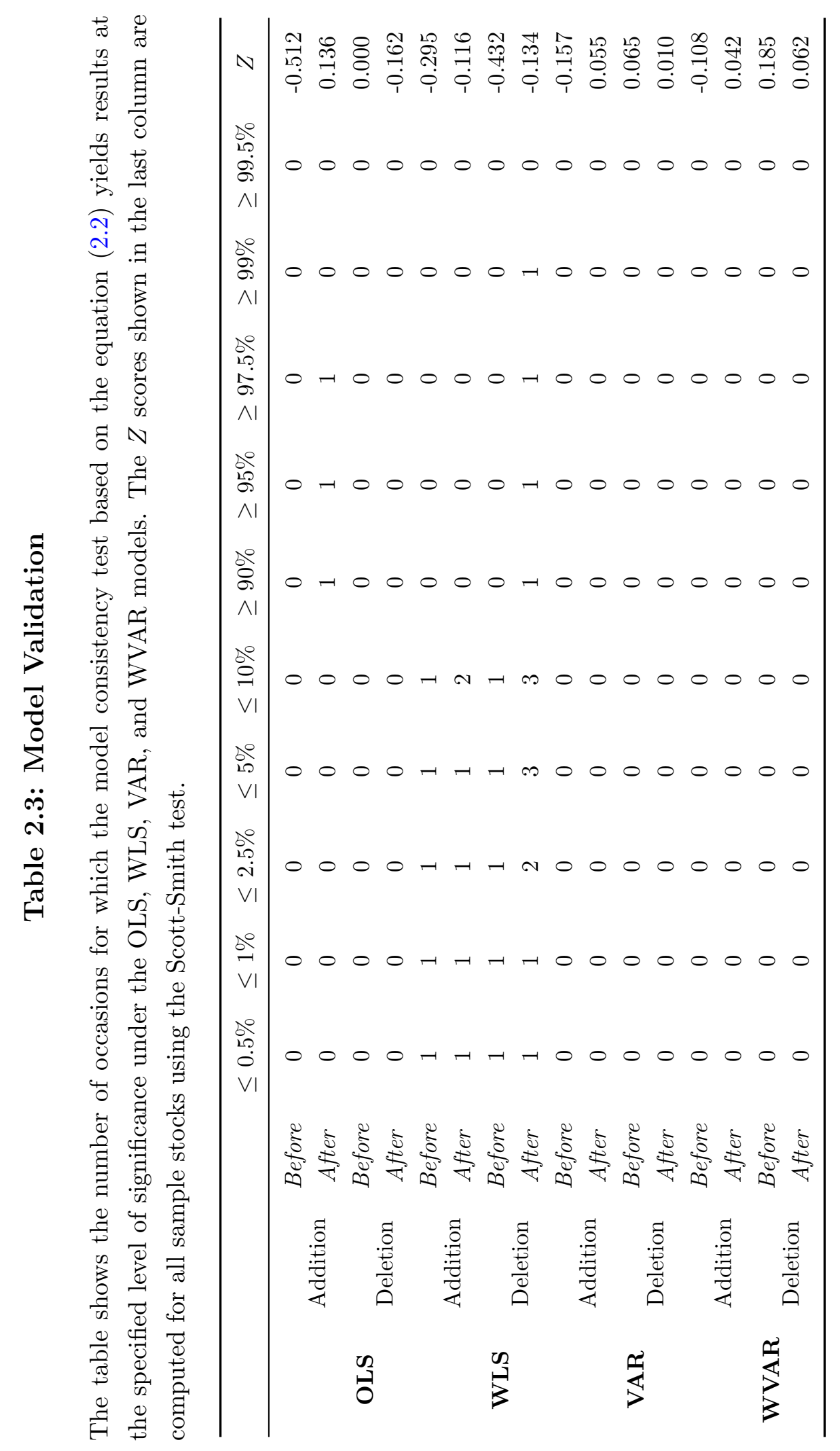


Table 2.4: Frequency of Changes in Model Parameters for Addition Events (OLS)

Based on the model defined at (2.1) using 60 days of trade data by OLS. The first panel shows results for individual parameters. The second panel shows results for the sums of the $\alpha \mathrm{s}, \beta \mathrm{s}, \gamma \mathrm{s}$ and $\delta$ s respectively. The first vertical section show the numbers of stocks for which the corresponding parameter is significantly less than zero, neutral or significantly greater than zero before the addition event. The significance level is defined at the $1 \%$. The second vertical section shows the corresponding results after the addition event. The section headed "Chsq"shows the value of a Chi-squared statistic that is used to test the difference between the observed frequencies in each row. The last section shows the results for the differences in parameters during the addition events and it shows the numbers of stocks for which the corresponding parameter exhibits significant decrease, neutral change or significant increase in its magnitude during the addition events. Differences are calculated by absolute value of model parameters between after and before the addition events. Cells in which there is a substantial number of statistically significant changes (more than $10 \%$ of the sample size, 1,127 ) are shown in bold.

\begin{tabular}{ccccccccccc}
\hline \multicolumn{6}{c}{ Before Addition } & \multicolumn{2}{c}{ After Addition } & \multicolumn{2}{c}{ Chsq } & \multicolumn{2}{c}{ Difference } & (After minus Before) \\
& -ive & ntrl & + ve & -ive & ntrl & +ve & 2 DoF & sig. dec. & no sig. change & sig. inc. \\
\hline$\alpha_{0}$ & 76 & 1027 & 24 & 84 & 1012 & 31 & 0.496 & 7 & 1110 & 10 \\
$\alpha_{1}$ & 1054 & 73 & 0 & 1044 & 82 & 1 & 0.456 & 98 & 907 & $\mathbf{1 2 2}$ \\
$\alpha_{2}$ & 919 & 208 & 0 & 838 & 289 & 0 & 0.000 & 61 & 1013 & 53 \\
$\alpha_{3}$ & 668 & 454 & 5 & 590 & 536 & 1 & 0.001 & 32 & 1074 & 21 \\
$\alpha_{4}$ & 463 & 661 & 3 & 384 & 742 & 1 & 0.001 & 17 & 1094 & 16 \\
$\alpha_{5}$ & 281 & 840 & 6 & 238 & 885 & 4 & 0.077 & 9 & 1112 & 6 \\
$\beta_{0}$ & 1 & 103 & 1023 & 0 & 126 & 1001 & 0.170 & $\mathbf{1 8 4}$ & 673 & $\mathbf{2 7 0}$ \\
$\beta_{1}$ & 1 & 206 & 920 & 1 & 275 & 851 & 0.002 & 87 & 868 & $\mathbf{1 7 2}$ \\
$\beta_{2}$ & 3 & 419 & 705 & 0 & 502 & 625 & 0.000 & 35 & 1011 & 81 \\
$\beta_{3}$ & 5 & 653 & 469 & 6 & 726 & 395 & 0.006 & 20 & 1051 & 56 \\
$\beta_{4}$ & 3 & 842 & 282 & 2 & 866 & 259 & 0.469 & 10 & 1091 & 26 \\
$\beta_{5}$ & 10 & 935 & 182 & 8 & 969 & 150 & 0.141 & 7 & 1101 & 19 \\
$\gamma_{0}$ & 143 & 790 & 194 & 129 & 840 & 158 & 0.051 & 62 & 1019 & 46 \\
$\gamma_{1}$ & 262 & 739 & 126 & 274 & 773 & 80 & 0.004 & 73 & 1009 & 45 \\
$\gamma_{2}$ & 157 & 915 & 55 & 144 & 948 & 35 & 0.061 & 26 & 1079 & 22 \\
$\gamma_{3}$ & 76 & 1009 & 42 & 96 & 1000 & 31 & 0.134 & 14 & 1102 & 11 \\
$\gamma_{4}$ & 55 & 1033 & 39 & 40 & 1059 & 28 & 0.106 & 9 & 1116 & 2 \\
$\gamma_{5}$ & 13 & 1066 & 48 & 25 & 1072 & 30 & 0.019 & 10 & 1114 & 3 \\
$\delta_{1}$ & 1 & 148 & 978 & 1 & 186 & 940 & 0.079 & 45 & 1055 & 27 \\
$\delta_{2}$ & 0 & 345 & 782 & 3 & 424 & 700 & 0.000 & 15 & 1097 & 15 \\
$\delta_{3}$ & 0 & 540 & 587 & 1 & 626 & 500 & 0.001 & 17 & 1097 & 13 \\
$\delta_{4}$ & 0 & 668 & 459 & 1 & 740 & 386 & 0.004 & 10 & 1114 & 3 \\
$\delta_{5}$ & 0 & 742 & 385 & 0 & 796 & 331 & 0.051 & 10 & 1113 & 4 \\
$A$ & 1023 & 103 & 1 & 1006 & 121 & 0 & 0.274 & 89 & 950 & 88 \\
$B$ & 3 & 52 & 1072 & 1 & 65 & 1061 & 0.286 & $\mathbf{2 0 5}$ & 589 & $\mathbf{3 3 3}$ \\
$\Gamma$ & 186 & 816 & 125 & 186 & 861 & 80 & 0.004 & 57 & 1038 & 32 \\
$\Delta$ & 0 & 72 & 1055 & 1 & 87 & 1039 & 0.281 & 64 & 1019 & 44 \\
\hline & & & & & & & & & & \\
\end{tabular}




\section{Table 2.5: Frequency of Changes in Model Parameters for Deletion Events (OLS)}

Based on the model defined at (2.1) using 60 days of trade data by OLS. The first panel shows results for individual parameters. The second panel shows results for the sums of the $\alpha \mathrm{s}, \beta \mathrm{s}, \gamma_{\mathrm{s}}$ and $\delta$ s respectively. The first vertical section show the numbers of stocks for which the corresponding parameter is significantly less than zero, neutral or significantly greater than zero before the deletion event. The significance level is defined at the $1 \%$. The second vertical section shows the corresponding results after the deletion event. The section headed "Chsq"shows the value of a Chi-squared statistic that is used to test the difference between the observed frequencies in each row. The last section shows the results for the differences in parameters during the deletion events and it shows the numbers of stocks for which the corresponding parameter exhibits significant decrease, neutral change or significant increase in its magnitude during the deletion events. Differences are calculated by absolute value of model parameters between after and before the deletion events. Cells in which there is a substantial number of statistically significant changes (more than $10 \%$ of the sample size, 728 ) are shown in bold.

\begin{tabular}{|c|c|c|c|c|c|c|c|c|c|c|}
\hline & \multicolumn{3}{|c|}{ Before Deletion } & \multicolumn{3}{|c|}{ After Deletion } & \multirow{2}{*}{$\begin{array}{l}\text { Chsq } \\
2 \text { DoF }\end{array}$} & \multicolumn{2}{|c|}{ Difference (After minus } & \multirow{2}{*}{$\begin{array}{l}\text { Before) } \\
\text { sig. inc. }\end{array}$} \\
\hline & -ive & ntrl & $+\mathrm{ve}$ & -ive & ntrl & $+\mathrm{ve}$ & & sig. dec. & no sig. change & \\
\hline$\alpha_{0}$ & 30 & 681 & 17 & 26 & 685 & 17 & 0.862 & 3 & 723 & 2 \\
\hline$\alpha_{1}$ & 648 & 80 & 0 & 657 & 71 & 0 & 0.741 & 39 & 613 & 76 \\
\hline$\alpha_{2}$ & 471 & 257 & 0 & 504 & 224 & 0 & 0.184 & 17 & 670 & 41 \\
\hline$\alpha_{3}$ & 312 & 415 & 1 & 342 & 383 & 3 & 0.160 & 11 & 698 & 19 \\
\hline$\alpha_{4}$ & 159 & 568 & 1 & 216 & 512 & 0 & 0.002 & 7 & 708 & 13 \\
\hline$\alpha_{5}$ & 80 & 647 & 1 & 108 & 618 & 2 & 0.075 & 3 & 720 & 5 \\
\hline$\beta_{0}$ & 0 & 80 & 648 & 0 & 97 & 631 & 0.395 & 168 & 463 & 97 \\
\hline$\beta_{1}$ & 0 & 313 & 415 & 2 & 291 & 435 & 0.195 & 54 & 642 & 32 \\
\hline$\beta_{2}$ & 1 & 498 & 229 & 4 & 468 & 256 & 0.120 & 21 & 690 & 17 \\
\hline$\beta_{3}$ & 3 & 607 & 118 & 3 & 585 & 140 & 0.319 & 7 & 716 & 5 \\
\hline$\beta_{4}$ & 2 & 678 & 48 & 3 & 669 & 56 & 0.645 & 5 & 719 & 4 \\
\hline$\beta_{5}$ & 7 & 698 & 23 & 7 & 696 & 25 & 0.958 & 2 & 722 & 4 \\
\hline$\gamma_{0}$ & 108 & 555 & 65 & 68 & 589 & 71 & 0.006 & 19 & 682 & 27 \\
\hline$\gamma_{1}$ & 186 & 517 & 25 & 215 & 493 & 20 & 0.200 & 22 & 651 & 55 \\
\hline$\gamma_{2}$ & 72 & 648 & 8 & 100 & 622 & 6 & 0.068 & 6 & 705 & 17 \\
\hline$\gamma_{3}$ & 51 & 671 & 6 & 59 & 662 & 7 & 0.698 & 2 & 717 & 9 \\
\hline$\gamma_{4}$ & 23 & 700 & 5 & 28 & 694 & 6 & 0.738 & 1 & 726 & 1 \\
\hline$\gamma_{5}$ & 9 & 715 & 4 & 8 & 714 & 6 & 0.795 & 0 & 726 & 2 \\
\hline$\delta_{1}$ & 0 & 224 & 504 & 1 & 207 & 520 & 0.383 & 16 & 689 & 23 \\
\hline$\delta_{2}$ & 1 & 412 & 315 & 1 & 399 & 328 & 0.790 & 3 & 717 & 8 \\
\hline$\delta_{3}$ & 2 & 540 & 186 & 1 & 506 & 221 & 0.108 & 1 & 716 & 11 \\
\hline$\delta_{4}$ & 1 & 605 & 122 & 4 & 591 & 133 & 0.295 & 1 & 725 & 2 \\
\hline$\delta_{5}$ & 1 & 627 & 100 & 2 & 632 & 94 & 0.764 & 3 & 720 & 5 \\
\hline$A$ & 594 & 134 & 0 & 607 & 121 & 0 & 0.669 & 26 & 644 & 58 \\
\hline$B$ & 0 & 64 & 664 & 0 & 111 & 617 & 0.001 & 158 & 479 & 91 \\
\hline$\Gamma$ & 130 & 579 & 19 & 141 & 572 & 15 & 0.619 & 16 & 681 & 31 \\
\hline$\Delta$ & 0 & 115 & 613 & 1 & 129 & 598 & 0.370 & 18 & 678 & 32 \\
\hline
\end{tabular}




\section{Table 2.6: Frequency of Changes in Model Parameters for Addition Events (VAR)}

Based on the model defined at (2.1) using 60 days of trade data by VAR. The first panel shows results for individual parameters. The second panel shows results for the sums of the $\alpha \mathrm{s}, \beta \mathrm{s}, \gamma \mathrm{s}$ and $\delta$ s respectively. The first vertical section show the numbers of stocks for which the corresponding parameter is significantly less than zero, neutral or significantly greater than zero before the addition event. The significance level is defined at the $1 \%$. The second vertical section shows the corresponding results after the addition event. The section headed "Chsq" shows the value of a Chi-squared statistic that is used to test the difference between the observed frequencies in each row. The last section shows the results for the differences in parameters during the addition events and it shows the numbers of stocks for which the corresponding parameter exhibits significant decrease, neutral change or significant increase in its magnitude during the addition events. Differences are calculated by absolute value of model parameters between after and before the addition events. Cells in which there is a substantial number of statistically significant changes (more than $10 \%$ of the sample size, 1,127 ) are shown in bold.

\begin{tabular}{|c|c|c|c|c|c|c|c|c|c|c|}
\hline & Befor & e Adc & ition & After & Add & tion & Chsq & Differen & e (After minus & Before) \\
\hline & -ive & ntrl & $+\mathrm{ve}$ & -ive & ntrl & $+\mathrm{ve}$ & 2 DoF & sig. dec. & no sig. change & sig. inc. \\
\hline$\alpha_{0}$ & 68 & 1044 & 15 & 76 & 1034 & 17 & 0.734 & 6 & 1108 & 13 \\
\hline$\alpha_{1}$ & 1047 & 79 & 1 & 1041 & 86 & 0 & 0.518 & 184 & 773 & 170 \\
\hline$\alpha_{2}$ & 939 & 188 & 0 & 896 & 230 & 1 & 0.044 & 123 & 899 & 105 \\
\hline$\alpha_{3}$ & 741 & 386 & 0 & 669 & 448 & 10 & 0.000 & 80 & 992 & 55 \\
\hline$\alpha_{4}$ & 534 & 584 & 9 & 450 & 666 & 11 & 0.002 & 48 & 1035 & 44 \\
\hline$\alpha_{5}$ & 350 & 765 & 12 & 291 & 822 & 14 & 0.022 & 20 & 1083 & 24 \\
\hline$\beta_{0}$ & 0 & 92 & 1035 & 1 & 108 & 1018 & 0.298 & 187 & 655 & 285 \\
\hline$\beta_{1}$ & 2 & 165 & 960 & 1 & 223 & 903 & 0.005 & 141 & 733 & 253 \\
\hline$\beta_{2}$ & 3 & 414 & 710 & 0 & 489 & 638 & 0.001 & 50 & 949 & 128 \\
\hline$\beta_{3}$ & 5 & 657 & 465 & 8 & 700 & 419 & 0.108 & 35 & 1010 & 82 \\
\hline$\beta_{4}$ & 4 & 818 & 305 & 4 & 851 & 272 & 0.281 & 14 & 1071 & 42 \\
\hline$\beta_{5}$ & 10 & 922 & 195 & 19 & 936 & 172 & 0.114 & 8 & 1085 & 34 \\
\hline$\gamma_{0}$ & 155 & 784 & 188 & 151 & 821 & 155 & 0.130 & 55 & 1021 & 51 \\
\hline$\gamma_{1}$ & 271 & 725 & 131 & 253 & 781 & 93 & 0.010 & 80 & 996 & 51 \\
\hline$\gamma_{2}$ & 142 & 919 & 66 & 135 & 955 & 37 & 0.011 & 39 & 1063 & 25 \\
\hline$\gamma_{3}$ & 68 & 1016 & 43 & 79 & 1017 & 31 & 0.250 & 18 & 1098 & 11 \\
\hline$\gamma_{4}$ & 47 & 1037 & 43 & 38 & 1047 & 42 & 0.603 & 12 & 1110 & 5 \\
\hline$\gamma_{5}$ & 9 & 1068 & 50 & 14 & 1083 & 30 & 0.045 & 14 & 1108 & 5 \\
\hline$\delta_{1}$ & 1 & 144 & 982 & 0 & 172 & 955 & 0.145 & 116 & 929 & 82 \\
\hline$\delta_{2}$ & 3 & 212 & 912 & 3 & 288 & 836 & 0.001 & 93 & 974 & 60 \\
\hline$\delta_{3}$ & 1 & 384 & 742 & 1 & 466 & 660 & 0.002 & 66 & 1014 & 47 \\
\hline$\delta_{4}$ & 10 & 531 & 586 & 4 & 612 & 511 & 0.001 & 58 & 1026 & 43 \\
\hline$\delta_{5}$ & 5 & 616 & 506 & 5 & 666 & 456 & 0.103 & 39 & 1048 & 40 \\
\hline$A$ & 1069 & 58 & 0 & 1060 & 66 & 1 & 0.460 & 174 & 778 & 175 \\
\hline$B$ & 1 & 60 & 1066 & 0 & 65 & 1062 & 0.547 & 208 & 597 & 322 \\
\hline$\Gamma$ & 180 & 820 & 127 & 171 & 877 & 79 & 0.001 & 70 & 1022 & 35 \\
\hline$\Delta$ & 0 & 38 & 1089 & 0 & 52 & 1075 & 0.322 & 181 & 823 & 123 \\
\hline
\end{tabular}


Table 2.7: Frequency of Changes in Model Parameters for Deletion Events (VAR)

Based on the model defined at (2.1) using 60 days of trade data by VAR. The first panel shows results for individual parameters. The second panel shows results for the sums of the $\alpha \mathrm{s}, \beta_{\mathrm{s}}, \gamma_{\mathrm{s}}$ and $\delta \mathrm{s}$ respectively. The first vertical section show the numbers of stocks for which the corresponding parameter is significantly less than zero, neutral or significantly greater than zero before the deletion event. The significance level is defined at the $1 \%$. The second vertical section shows the corresponding results after the deletion event. The section headed "Chsq"shows the value of a Chi-squared statistic that is used to test the difference between the observed frequencies in each row. The last section shows the results for the differences in parameters during the deletion events and it shows the numbers of stocks for which the corresponding parameter exhibits significant decrease, neutral change or significant increase in its magnitude during the deletion events. Differences are calculated by absolute value of model parameters between after and before the deletion events. Cells in which there is a substantial number of statistically significant changes (more than $10 \%$ of the sample size, 728 ) are shown in bold.

\begin{tabular}{|c|c|c|c|c|c|c|c|c|c|c|}
\hline & \multicolumn{3}{|c|}{ Before Deletion } & \multicolumn{3}{|c|}{ After Deletion } & \multirow{2}{*}{$\begin{array}{l}\text { Chsq } \\
2 \text { DoF }\end{array}$} & \multicolumn{2}{|c|}{ Difference (After minus } & \multirow{2}{*}{$\begin{array}{l}\text { Before) } \\
\text { sig. inc. }\end{array}$} \\
\hline & -ive & ntrl & $+\mathrm{ve}$ & -ive & ntrl & $+\mathrm{ve}$ & & sig. dec. & no sig. change & \\
\hline$\alpha_{0}$ & 23 & 693 & 12 & 29 & 689 & 10 & 0.642 & 1 & 727 & 0 \\
\hline$\alpha_{1}$ & 602 & 125 & 1 & 598 & 129 & 1 & 0.963 & 67 & 551 & 110 \\
\hline$\alpha_{2}$ & 462 & 266 & 0 & 486 & 242 & 0 & 0.419 & 47 & 612 & 69 \\
\hline$\alpha_{3}$ & 332 & 392 & 4 & 342 & 384 & 2 & 0.638 & 34 & 662 & 32 \\
\hline$\alpha_{4}$ & 184 & 538 & 6 & 228 & 499 & 1 & 0.008 & 16 & 682 & 30 \\
\hline$\alpha_{5}$ & 124 & 598 & 6 & 128 & 597 & 3 & 0.587 & 7 & 710 & 11 \\
\hline$\beta_{0}$ & 0 & 70 & 658 & 0 & 89 & 639 & 0.280 & 177 & 447 & 104 \\
\hline$\beta_{1}$ & 0 & 270 & 458 & 3 & 269 & 456 & 0.222 & 70 & 607 & 51 \\
\hline$\beta_{2}$ & 5 & 489 & 234 & 3 & 463 & 262 & 0.248 & 35 & 675 & 18 \\
\hline$\beta_{3}$ & 2 & 613 & 113 & 3 & 591 & 134 & 0.303 & 7 & 712 & 9 \\
\hline$\beta_{4}$ & 8 & 667 & 53 & 5 & 674 & 49 & 0.642 & 6 & 717 & 5 \\
\hline$\beta_{5}$ & 7 & 703 & 18 & 9 & 701 & 18 & 0.881 & 2 & 724 & 2 \\
\hline$\gamma_{0}$ & 125 & 542 & 61 & 85 & 573 & 70 & 0.011 & 19 & 679 & 30 \\
\hline$\gamma_{1}$ & 191 & 514 & 23 & 200 & 508 & 20 & 0.798 & 30 & 634 & 64 \\
\hline$\gamma_{2}$ & 73 & 645 & 10 & 85 & 634 & 9 & 0.589 & 6 & 699 & 23 \\
\hline$\gamma_{3}$ & 38 & 680 & 10 & 45 & 681 & 2 & 0.052 & 3 & 718 & 7 \\
\hline$\gamma_{4}$ & 20 & 698 & 10 & 21 & 700 & 7 & 0.757 & 4 & 722 & 2 \\
\hline$\gamma_{5}$ & 8 & 714 & 6 & 11 & 710 & 7 & 0.755 & 4 & 722 & 2 \\
\hline$\delta_{1}$ & 0 & 311 & 417 & 0 & 286 & 442 & 0.412 & 26 & 677 & 25 \\
\hline$\delta_{2}$ & 4 & 305 & 419 & 1 & 291 & 436 & 0.291 & 27 & 672 & 29 \\
\hline$\delta_{3}$ & 5 & 454 & 269 & 2 & 438 & 288 & 0.329 & 27 & 673 & 28 \\
\hline$\delta_{4}$ & 3 & 533 & 192 & 4 & 524 & 200 & 0.826 & 17 & 696 & 15 \\
\hline$\delta_{5}$ & 5 & 568 & 155 & 5 & 567 & 156 & 0.998 & 11 & 702 & 15 \\
\hline$A$ & 658 & 69 & 1 & 658 & 70 & 0 & 0.604 & 75 & 525 & 128 \\
\hline$B$ & 0 & 65 & 663 & 0 & 96 & 632 & 0.035 & 159 & 477 & 92 \\
\hline$\Gamma$ & 116 & 590 & 22 & 125 & 585 & 18 & 0.685 & 16 & 683 & 29 \\
\hline$\Delta$ & 0 & 66 & 662 & 0 & 82 & 646 & 0.382 & 66 & 574 & 88 \\
\hline
\end{tabular}


Table 2.8: Summary of the Differences in

\section{Frequency of Changes in Model Parameters for Addition Events}

The table reports a summary of the difference in frequency of changes in model parameters for the addition events. The significance level is defined as the $1 \%$.

\begin{tabular}{|c|c|c|c|c|c|c|c|c|c|}
\hline & \multicolumn{3}{|c|}{ Before Addition } & \multicolumn{3}{|c|}{ After Addition } & \multicolumn{2}{|c|}{ Difference (After minus } & \multirow{2}{*}{$\begin{array}{l}\text { Before) } \\
\text { sig. inc. }\end{array}$} \\
\hline & -ive & ntrl & $+\mathrm{ve}$ & -ive & ntrl & $+\mathrm{ve}$ & sig. dec. & no sig. change & \\
\hline \multicolumn{10}{|c|}{ (i) OLS-WLS } \\
\hline$\beta_{0}$ & 1 & 113 & -114 & 1 & 143 & -144 & -78 & 165 & -87 \\
\hline$\beta_{1}$ & 1 & 285 & -286 & 1 & 280 & -281 & -52 & 145 & -93 \\
\hline$B$ & -1 & 61 & -60 & -1 & 88 & -87 & -76 & 176 & -100 \\
\hline \multicolumn{10}{|c|}{ (ii) VAR-WVAR } \\
\hline$\beta_{0}$ & 0 & 97 & -97 & 0 & 140 & -140 & -83 & 174 & -91 \\
\hline$\delta_{1}$ & -1 & -41 & 42 & 4 & -45 & 41 & 123 & -220 & 97 \\
\hline$\delta_{2}$ & 3 & 84 & -87 & 14 & 44 & -58 & 40 & -115 & 75 \\
\hline$B$ & 0 & 48 & -48 & 0 & 88 & -88 & -78 & 163 & -85 \\
\hline$\Delta$ & 0 & 29 & -29 & 2 & 41 & -43 & 70 & -124 & 54 \\
\hline \multicolumn{10}{|c|}{ (iii) OLS-VAR } \\
\hline$\alpha_{1}$ & -7 & 6 & 1 & -3 & 4 & -1 & 86 & -134 & 48 \\
\hline$\alpha_{2}$ & 20 & -20 & 0 & 58 & -59 & 1 & 62 & -114 & 52 \\
\hline$\beta_{1}$ & 1 & -41 & 40 & 0 & -52 & 52 & 54 & -135 & 81 \\
\hline$\delta_{1}$ & 0 & -4 & 4 & -1 & -14 & 15 & 71 & -126 & 55 \\
\hline$\delta_{2}$ & 3 & -133 & 130 & 0 & -136 & 136 & 78 & -123 & 45 \\
\hline$A$ & 46 & -45 & -1 & 54 & -55 & 1 & 85 & -172 & 87 \\
\hline$\Delta$ & 0 & -34 & 34 & -1 & -35 & 36 & 117 & -196 & 79 \\
\hline \multicolumn{10}{|c|}{ (iv) WLS-WVAR } \\
\hline$\alpha_{1}$ & -66 & 64 & 2 & -54 & 52 & 2 & 68 & -120 & 52 \\
\hline$\beta_{1}$ & 1 & -209 & 208 & 3 & -196 & 193 & 93 & -239 & 146 \\
\hline$\beta_{2}$ & 7 & -183 & 176 & 5 & -187 & 182 & 39 & -134 & 95 \\
\hline$\gamma_{1}$ & 47 & -122 & 75 & 37 & -124 & 87 & 82 & -128 & 46 \\
\hline$\delta_{1}$ & 0 & -200 & 200 & 4 & -213 & 209 & 206 & -357 & 151 \\
\hline$\delta_{2}$ & 5 & -247 & 242 & 15 & -275 & 260 & 120 & -243 & 123 \\
\hline$\delta_{3}$ & 4 & -248 & 244 & 9 & -272 & 263 & 85 & -167 & 82 \\
\hline$\delta_{4}$ & 4 & -247 & 243 & 7 & -229 & 222 & 73 & -154 & 81 \\
\hline$\delta_{5}$ & 9 & -223 & 214 & 11 & -197 & 186 & 59 & -131 & 72 \\
\hline$A$ & 83 & -84 & 1 & 149 & -154 & 5 & 94 & -165 & 71 \\
\hline$\Delta$ & 0 & -65 & 65 & 2 & -63 & 61 & 191 & -331 & 140 \\
\hline
\end{tabular}


Table 2.9: Summary of the Differences in Frequency of Changes in Model Parameters for Deletion Events

The table reports a summary of the difference in frequency of changes in model parameters for the deletion events. The significance level is defined as the $1 \%$.

\begin{tabular}{|c|c|c|c|c|c|c|c|c|c|}
\hline & \multicolumn{3}{|c|}{ Before Deletion } & \multicolumn{3}{|c|}{ After Deletion } & \multicolumn{2}{|c|}{ Difference (After minus } & \multirow{2}{*}{$\begin{array}{l}\text { Before) } \\
\text { sig. inc. }\end{array}$} \\
\hline & -ive & ntrl & + ve & -ive & ntrl & + ve & sig. dec. & no sig. change & \\
\hline \multicolumn{10}{|c|}{ (i) OLS-WLS } \\
\hline$\beta_{0}$ & 0 & 124 & -124 & 1 & 123 & -124 & -83 & 118 & -35 \\
\hline$B$ & 0 & 120 & -120 & 2 & 76 & -78 & -61 & 90 & -29 \\
\hline \multicolumn{10}{|c|}{ (ii) VAR-WVAR } \\
\hline$\beta_{0}$ & 0 & 114 & -114 & 1 & 100 & -101 & -82 & 117 & -35 \\
\hline$\delta_{1}$ & 4 & -141 & 137 & 2 & -116 & 114 & 84 & -170 & 86 \\
\hline$A$ & -166 & 161 & 5 & -166 & 162 & 4 & -21 & 73 & -52 \\
\hline$B$ & 0 & 113 & -113 & 1 & 88 & -89 & -66 & 98 & -32 \\
\hline \multicolumn{10}{|c|}{ (iii) OLS-VAR } \\
\hline$A$ & 64 & -65 & 1 & 51 & -51 & 0 & 49 & -119 & 70 \\
\hline$\Delta$ & 0 & -49 & 49 & -1 & -47 & 48 & 48 & -104 & 56 \\
\hline \multicolumn{10}{|c|}{ (iv) WLS-WVAR } \\
\hline$\alpha_{1}$ & -25 & 24 & 1 & -6 & 5 & 1 & 36 & -86 & 50 \\
\hline$\beta_{1}$ & 3 & -141 & 138 & 4 & -130 & 126 & 54 & -98 & 44 \\
\hline$\delta_{1}$ & 3 & -152 & 149 & 1 & -160 & 159 & 91 & -174 & 83 \\
\hline$\delta_{2}$ & 12 & -176 & 164 & 2 & -171 & 169 & 52 & -106 & 54 \\
\hline$A$ & 112 & -118 & 6 & 94 & -98 & 4 & 41 & -93 & 52 \\
\hline$\Delta$ & 2 & -96 & 94 & 0 & -91 & 91 & 61 & -155 & 94 \\
\hline
\end{tabular}




\section{Table 2.10: Parameter Dynamics (OLS)}

Based on the model defined at (2.1) using 60 days of trade data by OLS. Each panel shows the results of estimates of the sums of the $\alpha \mathrm{s}, \beta \mathrm{s}, \gamma_{\mathrm{s}}$ and $\delta \mathrm{s}$ respectively. Rows of each panel are before the event and columns are after it. "N" and "P" denotes negative and positive respectively. All other changes at a significance level of which is greater than $5 \%$ are classified as neutral. Bold entries in the table are percentages which are substantial. More detailed tables which cover individual parameters are available on request.

\begin{tabular}{|c|c|c|c|c|c|c|c|c|c|c|}
\hline \multicolumn{6}{|c|}{ Addition } & \multicolumn{5}{|c|}{ Deletion } \\
\hline & $\mathrm{N}(1 \%)$ & $\mathrm{N}(5 \%)$ & Neutral & $\mathrm{P}(5 \%)$ & $\mathrm{P}(1 \%)$ & $\mathrm{N}(1 \%)$ & $\mathrm{N}(5 \%)$ & Neutral & $\mathrm{P}(5 \%)$ & $\mathrm{P}(1 \%)$ \\
\hline \multicolumn{11}{|c|}{$A$} \\
\hline $\mathrm{N}(1 \%)$ & 83.85 & 2.84 & 4.08 & 0.00 & 0.00 & 70.88 & 5.49 & 5.22 & 0.00 & 0.00 \\
\hline $\mathrm{N}(5 \%)$ & 3.19 & 0.44 & 1.24 & 0.00 & 0.00 & 5.63 & 1.10 & 1.92 & 0.00 & 0.00 \\
\hline Neutral & 2.22 & 0.53 & 1.51 & 0.00 & 0.00 & 6.87 & 1.24 & 1.65 & 0.00 & 0.00 \\
\hline $\mathrm{P}(5 \%)$ & 0.00 & 0.00 & 0.00 & 0.00 & 0.00 & 0.00 & 0.00 & 0.00 & 0.00 & 0.00 \\
\hline $\mathrm{P}(1 \%)$ & 0.00 & 0.00 & 0.09 & 0.00 & 0.00 & 0.00 & 0.00 & 0.00 & 0.00 & 0.00 \\
\hline \multicolumn{11}{|c|}{$B$} \\
\hline $\mathrm{N}(1 \%)$ & 0.00 & 0.00 & 0.09 & 0.09 & 0.09 & 0.00 & 0.00 & 0.00 & 0.00 & 0.00 \\
\hline $\mathrm{N}(5 \%)$ & 0.00 & 0.00 & 0.00 & 0.00 & 0.00 & 0.00 & 0.00 & 0.00 & 0.00 & 0.00 \\
\hline Neutral & 0.09 & 0.00 & 0.98 & 0.80 & 1.33 & 0.00 & 0.00 & 2.75 & 1.24 & 2.34 \\
\hline $\mathrm{P}(5 \%)$ & 0.00 & 0.00 & 0.44 & 0.35 & 0.62 & 0.00 & 0.00 & 0.96 & 0.14 & 1.37 \\
\hline $\mathrm{P}(1 \%)$ & 0.00 & 0.00 & 1.95 & 1.06 & 92.10 & 0.00 & 0.00 & 6.32 & 3.85 & 81.04 \\
\hline \multicolumn{11}{|c|}{$\Gamma$} \\
\hline $\mathrm{N}(1 \%)$ & 7.99 & 1.69 & 6.65 & 0.09 & 0.09 & 8.52 & 0.96 & 8.38 & 0.00 & 0.00 \\
\hline $\mathrm{N}(5 \%)$ & 1.33 & 1.15 & 3.64 & 0.18 & 0.09 & 2.47 & 1.51 & 6.32 & 0.14 & 0.27 \\
\hline Neutral & 6.65 & 4.88 & 45.87 & 1.33 & 2.40 & 8.24 & 7.01 & 47.94 & 1.65 & 1.24 \\
\hline $\mathrm{P}(5 \%)$ & 0.35 & 0.18 & 3.19 & 0.80 & 0.35 & 0.14 & 0.14 & 1.92 & 0.27 & 0.27 \\
\hline $\mathrm{P}(1 \%)$ & 0.18 & 0.27 & 6.03 & 0.44 & 4.17 & 0.00 & 0.14 & 1.92 & 0.27 & 0.27 \\
\hline \multicolumn{11}{|c|}{$\Delta$} \\
\hline $\mathrm{N}(1 \%)$ & 0.00 & 0.00 & 0.00 & 0.00 & 0.00 & 0.00 & 0.00 & 0.00 & 0.00 & 0.00 \\
\hline $\mathrm{N}(5 \%)$ & 0.00 & 0.00 & 0.00 & 0.00 & 0.00 & 0.00 & 0.00 & 0.00 & 0.00 & 0.00 \\
\hline Neutral & 0.09 & 0.00 & 0.62 & 0.35 & 2.66 & 0.00 & 0.14 & 2.61 & 1.24 & 5.49 \\
\hline $\mathrm{P}(5 \%)$ & 0.00 & 0.00 & 0.44 & 0.18 & 2.04 & 0.00 & 0.00 & 1.24 & 1.24 & 3.85 \\
\hline $\mathrm{P}(1 \%)$ & 0.00 & 0.00 & 4.26 & 1.86 & 87.49 & 0.14 & 0.00 & 7.69 & 3.57 & 72.80 \\
\hline
\end{tabular}




\section{Table 2.11: Summary of Parameter Dynamics}

The tables shows the percentages in the specified dominant cells in the OLS dynamics in Table 2.10 and the comparable percentages for the WLS, VAR and WVAR models.

\begin{tabular}{cccccc}
\hline & & OLS & WLS & VAR & WVAR \\
\hline Parameter & Cell & \multicolumn{4}{c}{ Addition } \\
$A$ & $\mathrm{~N}(1 \%)-\mathrm{N}(1 \%)$ & 83.85 & 59.54 & 91.13 & 71.78 \\
$B$ & $\mathrm{P}(1 \%)-\mathrm{P}(1 \%)$ & 92.10 & 81.63 & 91.57 & 81.46 \\
$\Gamma$ & Neutral-Neutral & 45.87 & 44.54 & 48.09 & 37.53 \\
$\Delta$ & $\mathrm{P}(1 \%)-\mathrm{P}(1 \%)$ & 87.49 & 77.99 & 93.17 & 87.58 \\
& & \multicolumn{4}{c}{ Deletion } \\
$A$ & $\mathrm{~N}(1 \%)-\mathrm{N}(1 \%)$ & 70.88 & 37.23 & 83.52 & 53.02 \\
$B$ & $\mathrm{P}(1 \%)-\mathrm{P}(1 \%)$ & 81.04 & 60.85 & 82.28 & 62.23 \\
$\Gamma$ & Neutral-Neutral & 47.94 & 55.91 & 53.16 & 52.61 \\
$\Delta$ & $\mathrm{P} \mathrm{(1 \% )-P} \mathrm{(1 \% )}$ & 72.80 & 56.87 & 81.59 & 75.14 \\
\hline
\end{tabular}




\section{Table 2.12: $Z$ Scores for Tests of Difference in Model Parameters}

Based on the model defined at (2.1) using 60 days of trade data by four estimation methods. Differences are calculated by absolute value of model parameters between after and before both events (After minus Before). $Z$ scores for difference in individual parameters and sums are computed as described in Section 2.3.3. Bold format denotes significance at the $1 \%$ level.

\begin{tabular}{|c|c|c|c|c|c|c|c|c|}
\hline \multicolumn{5}{|c|}{ Addition } & \multicolumn{4}{|c|}{ Deletion } \\
\hline & OLS & WLS & VAR & WVAR & OLS & WLS & VAR & WVAR \\
\hline$\alpha_{0}$ & -0.113 & 2.202 & 1.014 & -0.609 & 1.325 & -0.756 & -1.025 & -0.297 \\
\hline$\alpha_{1}$ & -0.678 & 1.246 & -0.006 & 0.001 & 4.866 & 0.839 & 0.005 & 0.000 \\
\hline$\alpha_{2}$ & -2.404 & -1.220 & -0.008 & 0.000 & 2.374 & 0.174 & 0.004 & 0.000 \\
\hline$\alpha_{3}$ & -1.311 & -3.348 & -0.020 & -0.006 & 2.088 & 1.132 & 0.000 & 0.000 \\
\hline$\alpha_{4}$ & 0.147 & -0.306 & -0.015 & -0.003 & 3.810 & 0.205 & 0.005 & 0.000 \\
\hline$\alpha_{5}$ & 1.249 & 2.060 & 0.004 & -0.001 & 2.616 & -0.906 & 0.002 & 0.000 \\
\hline$\beta_{0}$ & 2.488 & 3.219 & 1.947 & 2.492 & 1.335 & 2.380 & 0.460 & 1.787 \\
\hline$\beta_{1}$ & -3.281 & 0.844 & 0.334 & -0.547 & 0.660 & 1.882 & 2.395 & 1.715 \\
\hline$\beta_{2}$ & 0.695 & -2.560 & 2.743 & 1.578 & 1.531 & -0.595 & 0.691 & 0.254 \\
\hline$\beta_{3}$ & 0.813 & 1.263 & 1.805 & 1.364 & 0.668 & 0.852 & 0.039 & -0.390 \\
\hline$\beta_{4}$ & -0.192 & 1.750 & -0.199 & -0.410 & 1.277 & 1.009 & 0.261 & 0.025 \\
\hline$\beta_{5}$ & -1.720 & 0.556 & 0.107 & 1.035 & 0.363 & 1.686 & 0.234 & 0.197 \\
\hline$\gamma_{0}$ & -1.241 & -0.510 & -1.392 & -0.413 & -2.276 & 2.681 & -1.739 & 2.867 \\
\hline$\gamma_{1}$ & 6.854 & -5.951 & 8.273 & -10.457 & 5.395 & -3.174 & 5.470 & -5.177 \\
\hline$\gamma_{2}$ & 3.212 & -3.422 & 3.549 & -4.582 & 4.251 & -2.488 & 4.093 & -3.316 \\
\hline$\gamma_{3}$ & 3.793 & -3.480 & 4.136 & & 4.302 & -0.934 & 3.719 & -1.233 \\
\hline$\gamma_{4}$ & 2.665 & -2.313 & 2.469 & -3.550 & 2.584 & -1.520 & 2.319 & -1.603 \\
\hline$\gamma_{5}$ & -1.021 & -2.702 & -1.619 & -4.685 & 1.552 & 0.275 & 1.299 & -0.134 \\
\hline$\delta_{1}$ & -0.766 & -2.409 & -0.138 & -5.163 & 0.931 & -0.045 & 0.380 & -0.176 \\
\hline$\delta_{2}$ & -3.541 & -2.058 & -3.292 & -4.937 & 0.367 & 1.275 & -0.081 & 2.976 \\
\hline$\delta_{3}$ & -2.535 & -1.030 & -4.872 & -3.010 & 1.780 & -0.461 & 2.702 & 0.039 \\
\hline$\delta_{4}$ & 0.605 & -1.640 & -0.752 & -2.919 & -1.267 & -0.302 & -1.039 & 0.438 \\
\hline$\delta_{5}$ & -0.600 & -0.940 & -1.291 & -1.638 & -0.536 & -1.509 & 1.084 & -1.202 \\
\hline$A$ & -1.195 & -0.620 & -2.177 & -3.211 & 4.923 & 0.617 & 5.394 & 3.347 \\
\hline$B$ & 1.386 & 3.487 & 2.132 & 2.753 & 1.001 & 2.355 & 1.105 & 1.471 \\
\hline$\Gamma$ & 7.068 & -7.355 & 7.788 & -12.257 & 6.188 & -3.374 & 5.806 & -4.377 \\
\hline$\Delta$ & -3.484 & -4.489 & -5.772 & -9.804 & 0.754 & -0.354 & 2.565 & 1.032 \\
\hline
\end{tabular}




\section{Table 2.13: Residual Correlation Test}

Based on the model defined at (2.1) using 60 days of trade data by OLS, WLS, VAR and WVAR, the table reports the residual correlation test under four models. The first vertical section presents the number of stock/events with no less than 100 transactions before and after each addition and deletion event. The second section shows the percentage for which the residual correlation at the significance level of $1 \%$ under the number of observations shown in the first vertical section for each addition and deletion event.

\begin{tabular}{cccccccccc}
\hline & & \multicolumn{4}{c}{ No. of Observations } & \multicolumn{3}{c}{ Significant Correlation } \\
& & OLS & WLS & VAR & WVAR & OLS & WLS & VAR & WVAR \\
\hline \multirow{3}{*}{ Addition } & Before & 1,114 & 1,114 & 1,124 & 1,124 & $0.00 \%$ & $0.00 \%$ & $93.59 \%$ & $92.62 \%$ \\
& After & 1,108 & 1,108 & 1,125 & 1,125 & $0.00 \%$ & $0.00 \%$ & $92.71 \%$ & $90.84 \%$ \\
& Before & 716 & 716 & 726 & 726 & $0.00 \%$ & $0.00 \%$ & $92.84 \%$ & $90.08 \%$ \\
& After & 700 & 700 & 724 & 724 & $0.00 \%$ & $0.00 \%$ & $93.09 \%$ & $91.71 \%$ \\
\hline
\end{tabular}




\section{Table 2.14: Summary of Model Estimations}

The table reports the summary of model estimation using 60 days of trade data by OLS, WLS, VAR and WVAR for addition and deletion events. There are 1,127 addition stocks and 728 deletion stocks by using these four models. The table shows the percentage of changes at the specified level of significance under each model. These significant changes are counted only if the autocorrelations of parameters measuring the speed of price adjustment decrease (increase) during the addition (deletion) events.

\begin{tabular}{ccccc}
\hline & OLS & WLS & VAR & WVAR \\
\hline \multicolumn{5}{c}{ Addition } \\
$\leq 0.1 \%$ & $7.19 \%$ & $3.99 \%$ & $16.68 \%$ & $20.41 \%$ \\
$\leq 1.0 \%$ & $11.98 \%$ & $8.43 \%$ & $22.63 \%$ & $26.00 \%$ \\
$\leq 5.0 \%$ & $20.85 \%$ & $15.35 \%$ & $30.43 \%$ & $29.64 \%$ \\
$\leq 10.0 \%$ & $25.73 \%$ & $21.47 \%$ & $33.72 \%$ & $33.63 \%$ \\
$\leq 5.1 \%$ & $4.81 \%$ & $3.30 \%$ & $15.25 \%$ & $14.29 \%$ \\
$\leq 1.0 \%$ & $11.13 \%$ & $7.01 \%$ & $23.35 \%$ & $21.29 \%$ \\
$\leq 5.0 \%$ & $19.09 \%$ & $13.74 \%$ & $31.18 \%$ & $27.20 \%$ \\
$\leq 10.0 \%$ & $22.94 \%$ & $19.51 \%$ & $33.52 \%$ & $31.04 \%$ \\
\hline
\end{tabular}




\section{Appendix A}

\section{Consistency of the VAR Model}

Hasbrouck (1991) adopts the bivariate VAR model to examine the impact of traderelated information on prices. The model is

$$
\begin{aligned}
& R_{t}=\sum_{i=1}^{5} \alpha_{i} R_{t-i}+\sum_{i=0}^{5} \beta_{i} X_{t-i}+\varepsilon_{R t} \\
& X_{t}=\sum_{i=1}^{5} \gamma_{i} R_{t-i}+\sum_{i=1}^{5} \delta_{i} X_{t-i}+\varepsilon_{X t} .
\end{aligned}
$$

Using the notation $E\left(R_{t}\right)=\mu$ and $E\left(X_{t}\right)=\theta$ and assuming that $E\left(\varepsilon_{R}\right)=$ $E\left(\varepsilon_{X}\right)=0$ gives

$$
\begin{aligned}
& \mu=\sum_{i=1}^{5} \alpha_{i} \mu+\sum_{i=0}^{5} \beta_{i} \theta \\
& \theta=\sum_{i=1}^{5} \gamma_{i} \mu+\sum_{i=1}^{5} \delta_{i} \theta .
\end{aligned}
$$


Elimination of $\mu$ and $\theta$ gives

$$
\left(1-\sum_{i=1}^{5} \alpha_{i}\right)\left(1-\sum_{i=1}^{5} \delta_{i}\right)-\sum_{i=0}^{5} \beta_{i} \sum_{i=1}^{5} \gamma_{i}=0
$$

Using the empirical results from Hasbrouck (1991),

$$
\sum_{i=1}^{5} \alpha_{i}=-0.166, \quad \sum_{i=0}^{5} \beta_{i}=0.023, \quad \sum_{i=1}^{5} \gamma_{i}=-5.856, \quad \sum_{i=1}^{5} \delta_{i}=0.42
$$

or those from Chung et al. (2005),

$$
\sum_{i=1}^{5} \alpha_{i}=0.0097, \quad \sum_{i=0}^{5} \beta_{i}=0.0915, \quad \sum_{i=1}^{5} \gamma_{i}=-1.06, \quad \sum_{i=1}^{5} \delta_{i}=0.4507
$$

and substituting them into (A.4) does not lead to zero, which may therefore be considered to be a contradiction. This may be resolved by specifying a constant term in each of the models at (A.1) and (A.2), as follows

$$
\begin{aligned}
& R_{t}=\alpha_{0}+\sum_{i=1}^{5} \alpha_{i} R_{t-i}+\sum_{i=0}^{5} \beta_{i} X_{t-i}+\varepsilon_{R, t} \\
& X_{t}=\gamma_{0}+\sum_{i=1}^{5} \gamma_{i} R_{t-i}+\sum_{i=1}^{5} \delta_{i} X_{t-i}+\varepsilon_{X, t}
\end{aligned}
$$

Taking expected values into (A.5) gives 


$$
\left(\left(1-\sum_{i=1}^{5} \alpha_{i}\right)\left(1-\sum_{i=1}^{5} \delta_{i}\right)-\sum_{i=0}^{5} \beta_{i} \sum_{i=1}^{5} \gamma_{i}\right) \mu=\left(1-\sum_{i=1}^{5} \delta_{i}\right) \alpha_{0}+\sum_{i=0}^{5} \beta_{i} \gamma_{0}
$$

which removes the inconsistency. 


\section{Appendix B}

\section{Test of Significance of $\Omega$ for the VAR Model Consistency}

The definition of $\Omega$ is written as

$$
\frac{\alpha_{0}+\gamma_{0}\left(\sum_{i=0}^{5} \beta_{i}\right) /\left(1-\sum_{i=1}^{5} \delta_{i}\right)}{\left\{1-\sum_{i=1}^{5} \alpha_{i}-\left(\sum_{i=0}^{5} \beta_{i}\right)\left(\sum_{i=1}^{5} \gamma_{i}\right) /\left(1-\sum_{i=1}^{5} \delta_{i}\right)\right\}}=\mu_{R}=\mu(\Theta) .
$$

The variance of $\mu(\hat{\Theta})$ is $\varphi^{T} \Sigma_{\hat{\Theta}} \varphi$ where $\varphi$ is the vector of first derivatives of $\mu(\Theta)$ with respect to the 23 elements of $\Theta$ and evaluated at $\hat{\Theta}$. Denoting the individual elements by $\varphi_{i}$, the vector is as

$$
\begin{aligned}
& \varphi_{1}=\Phi_{0}, \varphi_{k}=\Phi_{k}, k=2, \ldots, 6 ; \varphi_{k}=\Phi_{2}, k=7, \ldots, 12 ; \\
& \varphi_{13}=\Phi_{3}, \varphi_{k}=\Phi_{4}, k=14, \ldots, 18 ; \varphi_{k}=\Phi_{5}, k=19, \ldots, 23
\end{aligned}
$$

where

$$
\begin{gathered}
\Phi_{0}=1-\sum_{i=1}^{5} \alpha_{i}-\left(\sum_{i=0}^{5} \beta_{i}\right)\left(\sum_{i=1}^{5} \gamma_{i}\right) /\left(1-\sum_{i=1}^{5} \delta_{i}\right) \\
\Phi_{1}=-\mu_{R},
\end{gathered}
$$




$$
\begin{gathered}
\Phi_{2}=-\mu_{R}\left(\sum_{i=1}^{5} \gamma_{i}\right) /\left(1-\sum_{i=1}^{5} \delta_{i}\right)-\gamma_{0} /\left(1-\sum_{i=1}^{5} \delta_{i}\right) \\
\Phi_{3}=-\sum_{i=0}^{5} \beta_{i} /\left(1-\sum_{i=1}^{5} \delta_{i}\right), \\
\Phi_{4}=-\mu_{R}\left(\sum_{i=0}^{5} \beta_{i}\right) /\left(1-\sum_{i=1}^{5} \delta_{i}\right), \\
\Phi_{5}=-\mu_{R}\left(\sum_{i=1}^{5} \gamma_{i}\right)\left(\sum_{i=0}^{5} \beta_{i}\right) /\left(1-\sum_{i=1}^{5} \delta_{i}\right)^{2}-\gamma_{0}\left(\sum_{i=0}^{5} \beta_{i}\right) /\left(1-\sum_{i=1}^{5} \delta_{i}\right)^{2} .
\end{gathered}
$$


Appendix C

Other Tables 


\section{Table C.1: Frequency of Changes in Model Parameters for Addition Events (WLS)}

Based on the model defined at (2.1) using 60 days of trade data by WLS. The first panel shows results for individual parameters. The second panel shows results for the sums of the $\alpha \mathrm{s}, \beta \mathrm{s}, \gamma \mathrm{s}$ and $\delta$ s respectively. The first vertical section show the numbers of stocks for which the corresponding parameter is significantly less than zero, neutral or significantly greater than zero before the addition event. The significance level is defined at the $1 \%$. The second vertical section shows the corresponding results after the addition event. The section headed "Chsq" shows the value of a Chi-squared statistic that is used to test the difference between the observed frequencies in each row. The last section shows the results for the differences in parameters during the addition events and it shows the numbers of stocks for which the corresponding parameter exhibits significant decrease, neutral change or significant increase in its magnitude during the addition events. Differences are calculated by absolute value of model parameters between after and before the addition events. Cells in which there is a substantial number of statistically significant changes (more than $10 \%$ of the sample size, 1,127 ) are shown in bold.

\begin{tabular}{ccccccccccc}
\hline \multicolumn{6}{c}{ Before Addition } & \multicolumn{2}{c}{ After Addition } & \multicolumn{2}{c}{ Chsq } & \multicolumn{2}{c}{ Difference (After minus Before) } \\
& -ive & ntrl & + ve & -ive & ntrl & +ve & 2 DoF & sig. dec. & no sig. change & sig. inc. \\
\hline$\alpha_{0}$ & 22 & 1051 & 54 & 27 & 1059 & 41 & 0.314 & 6 & 1109 & 12 \\
$\alpha_{1}$ & 968 & 159 & 0 & 935 & 192 & 0 & 0.159 & 58 & 981 & 88 \\
$\alpha_{2}$ & 695 & 432 & 0 & 592 & 535 & 0 & 0.000 & 38 & 1056 & 33 \\
$\alpha_{3}$ & 421 & 704 & 2 & 309 & 809 & 9 & 0.000 & 19 & 1100 & 8 \\
$\alpha_{4}$ & 265 & 858 & 4 & 179 & 943 & 5 & 0.000 & 9 & 1115 & 3 \\
$\alpha_{5}$ & 137 & 988 & 2 & 104 & 1021 & 2 & 0.080 & 5 & 1117 & 5 \\
$\beta_{0}$ & 2 & 216 & 909 & 1 & 269 & 857 & 0.022 & 106 & 838 & $\mathbf{1 8 3}$ \\
$\beta_{1}$ & 2 & 491 & 634 & 2 & 555 & 570 & 0.026 & 35 & 1013 & 79 \\
$\beta_{2}$ & 2 & 753 & 372 & 3 & 799 & 325 & 0.094 & 14 & 1072 & 41 \\
$\beta_{3}$ & 5 & 904 & 218 & 1 & 930 & 196 & 0.122 & 6 & 1107 & 14 \\
$\beta_{4}$ & 4 & 989 & 134 & 4 & 1023 & 100 & 0.063 & 3 & 1116 & 8 \\
$\beta_{5}$ & 8 & 1016 & 103 & 3 & 1060 & 64 & 0.002 & 2 & 1115 & 10 \\
$\gamma_{0}$ & 63 & 742 & 322 & 75 & 785 & 267 & 0.025 & 98 & 982 & 47 \\
$\gamma_{1}$ & 148 & 673 & 306 & 149 & 728 & 250 & 0.020 & 70 & 1021 & 36 \\
$\gamma_{2}$ & 69 & 938 & 120 & 60 & 992 & 75 & 0.002 & 26 & 1085 & 16 \\
$\gamma_{3}$ & 25 & 1041 & 61 & 26 & 1060 & 41 & 0.128 & 15 & 1103 & 9 \\
$\gamma_{4}$ & 15 & 1063 & 49 & 10 & 1092 & 25 & 0.010 & 10 & 1112 & 5 \\
$\gamma_{5}$ & 7 & 1082 & 38 & 10 & 1089 & 28 & 0.356 & 8 & 1118 & 1 \\
$\delta_{1}$ & 0 & 303 & 824 & 0 & 340 & 787 & 0.226 & 33 & 1066 & 28 \\
$\delta_{2}$ & 1 & 543 & 583 & 2 & 607 & 518 & 0.021 & 13 & 1102 & 12 \\
$\delta_{3}$ & 3 & 697 & 427 & 1 & 778 & 348 & 0.001 & 14 & 1099 & 14 \\
$\delta_{4}$ & 1 & 828 & 298 & 1 & 868 & 258 & 0.148 & 8 & 1114 & 5 \\
$\delta_{5}$ & 2 & 851 & 274 & 1 & 884 & 242 & 0.229 & 14 & 1108 & 5 \\
$A$ & 848 & 278 & 1 & 765 & 361 & 1 & 0.001 & 53 & 1029 & 45 \\
$B$ & 2 & 113 & 1012 & 0 & 153 & 974 & 0.013 & $\mathbf{1 2 9}$ & 765 & $\mathbf{2 3 3}$ \\
$\Gamma$ & 81 & 783 & 263 & 76 & 835 & 216 & 0.040 & 72 & 1027 & 28 \\
$\Delta$ & 0 & 132 & 995 & 0 & 156 & 971 & 0.318 & 60 & 1030 & 37 \\
\hline & & & & & & & & & & \\
\end{tabular}




\section{Table C.2: Frequency of Changes in Model Parameters for Deletion Events (WLS)}

Based on the model defined at (2.1) using 60 days of trade data by WLS. The first panel shows results for individual parameters. The second panel shows results for the sums of the $\alpha \mathrm{s}, \beta \mathrm{s}, \gamma \mathrm{s}$ and $\delta$ s respectively. The first vertical section show the numbers of stocks for which the corresponding parameter is significantly less than zero, neutral or significantly greater than zero before the deletion event. The significance level is defined at the $1 \%$. The second vertical section shows the corresponding results after the deletion event. The section headed "Chsq"shows the value of a Chi-squared statistic that is used to test the difference between the observed frequencies in each row. The last section shows the results for the differences in parameters during the deletion events and it shows the numbers of stocks for which the corresponding parameter exhibits significant decrease, neutral change or significant increase in its magnitude during the deletion events. Differences are calculated by absolute value of model parameters between after and before the deletion events. Cells in which there is a substantial number of statistically significant changes (more than $10 \%$ of the sample size, 728 ) are shown in bold.

\begin{tabular}{|c|c|c|c|c|c|c|c|c|c|c|}
\hline & \multicolumn{3}{|c|}{ Before Deletion } & \multicolumn{3}{|c|}{ After Deletion } & \multirow{2}{*}{$\begin{array}{c}\text { Chsq } \\
2 \text { DoF }\end{array}$} & \multicolumn{2}{|c|}{ Difference (After minus } & \multirow{2}{*}{$\begin{array}{l}\text { Before) } \\
\text { sig. inc. }\end{array}$} \\
\hline & -ive & ntrl & $+\mathrm{ve}$ & -ive & ntrl & $+\mathrm{ve}$ & & sig. dec. & no sig. change & \\
\hline$\alpha_{0}$ & 13 & 682 & 33 & 16 & 681 & 31 & 0.830 & 7 & 716 & 5 \\
\hline$\alpha_{1}$ & 526 & 202 & 0 & 516 & 212 & 0 & 0.845 & 20 & 673 & 35 \\
\hline$\alpha_{2}$ & 285 & 441 & 2 & 298 & 430 & 0 & 0.297 & 11 & 704 & 13 \\
\hline$\alpha_{3}$ & 117 & 608 & 3 & 141 & 586 & 1 & 0.162 & 3 & 722 & 3 \\
\hline$\alpha_{4}$ & 58 & 666 & 4 & 74 & 651 & 3 & 0.324 & 2 & 724 & 2 \\
\hline$\alpha_{5}$ & 30 & 694 & 4 & 33 & 693 & 2 & 0.667 & 2 & 724 & 2 \\
\hline$\beta_{0}$ & 0 & 204 & 524 & 1 & 220 & 507 & 0.390 & 85 & 581 & 62 \\
\hline$\beta_{1}$ & 1 & 506 & 221 & 3 & 487 & 238 & 0.369 & 17 & 700 & 11 \\
\hline$\beta_{2}$ & 3 & 643 & 82 & 3 & 629 & 96 & 0.534 & 2 & 722 & 4 \\
\hline$\beta_{3}$ & 3 & 687 & 38 & 3 & 685 & 40 & 0.973 & 2 & 724 & 2 \\
\hline$\beta_{4}$ & 4 & 715 & 9 & 4 & 705 & 19 & 0.162 & 2 & 723 & 3 \\
\hline$\beta_{5}$ & 7 & 712 & 9 & 8 & 709 & 11 & 0.872 & 2 & 723 & 3 \\
\hline$\gamma_{0}$ & 73 & 547 & 108 & 45 & 562 & 121 & 0.023 & 25 & 671 & 32 \\
\hline$\gamma_{1}$ & 76 & 543 & 109 & 81 & 548 & 99 & 0.718 & 19 & 672 & 37 \\
\hline$\gamma_{2}$ & 20 & 677 & 31 & 35 & 667 & 26 & 0.100 & 4 & 715 & 9 \\
\hline$\gamma_{3}$ & 11 & 705 & 12 & 16 & 695 & 17 & 0.395 & 2 & 723 & 3 \\
\hline$\gamma_{4}$ & 6 & 711 & 11 & 10 & 708 & 10 & 0.590 & 1 & 725 & 2 \\
\hline$\gamma_{5}$ & 4 & 718 & 6 & 4 & 714 & 10 & 0.603 & 0 & 724 & 4 \\
\hline$\delta_{1}$ & 1 & 322 & 405 & 1 & 330 & 397 & 0.915 & 19 & 681 & 28 \\
\hline$\delta_{2}$ & 4 & 517 & 207 & 4 & 500 & 224 & 0.620 & 6 & 719 & 3 \\
\hline$\delta_{3}$ & 1 & 613 & 114 & 1 & 600 & 127 & 0.657 & 1 & 717 & 10 \\
\hline$\delta_{4}$ & 1 & 657 & 70 & 3 & 641 & 84 & 0.291 & 1 & 722 & 5 \\
\hline$\delta_{5}$ & 0 & 667 & 61 & 2 & 652 & 74 & 0.181 & 1 & 723 & 4 \\
\hline$A$ & 380 & 348 & 0 & 398 & 330 & 0 & 0.639 & 13 & 691 & 24 \\
\hline$B$ & 0 & 184 & 544 & 2 & 187 & 539 & 0.359 & 97 & 569 & 62 \\
\hline$\Gamma$ & 45 & 602 & 81 & 45 & 613 & 70 & 0.637 & 11 & 685 & 32 \\
\hline$\Delta$ & 0 & 188 & 540 & 1 & 208 & 519 & 0.297 & 29 & 666 & 33 \\
\hline
\end{tabular}




\section{Table C.3: Frequency of Changes in Model Parameters for Addition Events (WVAR)}

Based on the model defined at (2.1) using 60 days of trade data by WVAR. The first panel shows results for individual parameters. The second panel shows results for the sums of the $\alpha \mathrm{s}, \beta \mathrm{s}, \gamma \mathrm{s}$ and $\delta$ s respectively. The first vertical section show the numbers of stocks for which the corresponding parameter is significantly less than zero, neutral or significantly greater than zero before the addition event. The significance level is defined at the $1 \%$. The second vertical section shows the corresponding results after the addition event. The section headed "Chsq"shows the value of a Chi-squared statistic that is used to test the difference between the observed frequencies in each row. The last section shows the results for the differences in parameters during the addition events and it shows the numbers of stocks for which the corresponding parameter exhibits significant decrease, neutral change or significant increase in its magnitude during the addition events. Differences are calculated by absolute value of model parameters between after and before the addition events. Cells in which there is a substantial number of statistically significant changes (more than $10 \%$ of the sample size, 1,127 ) are shown in bold.

\begin{tabular}{|c|c|c|c|c|c|c|c|c|c|c|}
\hline & \multicolumn{3}{|c|}{ Before Addition } & \multicolumn{3}{|c|}{ After Addition } & \multirow{2}{*}{$\begin{array}{l}\text { Chsq } \\
2 \text { DoF }\end{array}$} & \multicolumn{2}{|c|}{ Difference (After minus } & \multirow{2}{*}{$\begin{array}{l}\text { Before) } \\
\text { sig. inc. }\end{array}$} \\
\hline & -ive & ntrl & $+\mathrm{ve}$ & -ive & ntrl & $+\mathrm{ve}$ & & sig. dec. & no sig. change & \\
\hline$\alpha_{0}$ & 18 & 1073 & 36 & 30 & 1053 & 44 & 0.136 & 5 & 1110 & 12 \\
\hline$\alpha_{1}$ & 902 & 223 & 2 & 881 & 244 & 2 & 0.551 & 126 & 861 & 140 \\
\hline$\alpha_{2}$ & 715 & 406 & 6 & 666 & 457 & 4 & 0.076 & 90 & 967 & 70 \\
\hline$\alpha_{3}$ & 474 & 644 & 9 & 356 & 743 & 28 & 0.000 & 51 & 1045 & 31 \\
\hline$\alpha_{4}$ & 277 & 825 & 25 & 220 & 880 & 27 & 0.015 & 33 & 1066 & 28 \\
\hline$\alpha_{5}$ & 150 & 947 & 30 & 112 & 991 & 24 & 0.028 & 15 & 1092 & 20 \\
\hline$\beta_{0}$ & 0 & 189 & 938 & 1 & 248 & 878 & 0.004 & 104 & 829 & 194 \\
\hline$\beta_{1}$ & 3 & 282 & 842 & 5 & 359 & 763 & 0.001 & 128 & 774 & 225 \\
\hline$\beta_{2}$ & 9 & 570 & 548 & 8 & 612 & 507 & 0.208 & 53 & 938 & 136 \\
\hline$\beta_{3}$ & 10 & 751 & 366 & 12 & 795 & 320 & 0.104 & 29 & 1019 & 79 \\
\hline$\beta_{4}$ & 12 & 867 & 248 & 13 & 909 & 205 & 0.078 & 19 & 1069 & 39 \\
\hline$\beta_{5}$ & 15 & 915 & 197 & 24 & 950 & 153 & 0.016 & 16 & 1056 & 55 \\
\hline$\gamma_{0}$ & 62 & 743 & 322 & 76 & 796 & 255 & 0.004 & 96 & 987 & 44 \\
\hline$\gamma_{1}$ & 195 & 551 & 381 & 186 & 604 & 337 & 0.069 & 152 & 893 & 82 \\
\hline$\gamma_{2}$ & 105 & 834 & 188 & 87 & 890 & 150 & 0.020 & 83 & 999 & 45 \\
\hline$\gamma_{3}$ & 39 & 955 & 133 & 28 & 1000 & 99 & 0.020 & 57 & 1048 & 22 \\
\hline$\gamma_{4}$ & 25 & 989 & 113 & 21 & 1026 & 80 & 0.036 & 44 & 1063 & 20 \\
\hline$\gamma_{5}$ & 11 & 1015 & 101 & 15 & 1043 & 69 & 0.030 & 45 & 1074 & 8 \\
\hline$\delta_{1}$ & 0 & 103 & 1024 & 4 & 127 & 996 & 0.032 & 239 & 709 & 179 \\
\hline$\delta_{2}$ & 6 & 296 & 825 & 17 & 332 & 778 & 0.013 & 133 & 859 & 135 \\
\hline$\delta_{3}$ & 7 & 449 & 671 & 10 & 506 & 611 & 0.034 & 99 & 932 & 96 \\
\hline$\delta_{4}$ & 5 & 581 & 541 & 8 & 639 & 480 & 0.029 & 81 & 960 & 86 \\
\hline$\delta_{5}$ & 11 & 628 & 488 & 12 & 687 & 428 & 0.037 & 73 & 977 & 77 \\
\hline$A$ & 931 & 194 & 2 & 914 & 207 & 6 & 0.276 & 147 & 864 & 116 \\
\hline$B$ & 1 & 108 & 1018 & 0 & 153 & 974 & 0.008 & 130 & 760 & 237 \\
\hline$\Gamma$ & 104 & 691 & 332 & 99 & 746 & 282 & 0.043 & 147 & 918 & 62 \\
\hline$\Delta$ & 0 & 67 & 1060 & 2 & 93 & 1032 & 0.037 & 251 & 699 & 177 \\
\hline
\end{tabular}




\section{Table C.4: Frequency of Changes in Model Parameters for Deletion Events (WVAR)}

Based on the model defined at (2.1) using 60 days of trade data by WVAR. The first panel shows results for individual parameters. The second panel shows results for the sums of the $\alpha \mathrm{s}, \beta \mathrm{s}, \gamma \mathrm{s}$ and $\delta \mathrm{s}$ respectively. The first vertical section show the numbers of stocks for which the corresponding parameter is significantly less than zero, neutral or significantly greater than zero before the deletion event. The significance level is defined at the $1 \%$. The second vertical section shows the corresponding results after the deletion event. The section headed "Chsq"shows the value of a Chi-squared statistic that is used to test the difference between the observed frequencies in each row. The last section shows the results for the differences in parameters during the deletion events and it shows the numbers of stocks for which the corresponding parameter exhibits significant decrease, neutral change or significant increase in its magnitude during the deletion events. Differences are calculated by absolute value of model parameters between after and before the deletion events. Cells in which there is a substantial number of statistically significant changes (more than $10 \%$ of the sample size, 728 ) are shown in bold.

\begin{tabular}{|c|c|c|c|c|c|c|c|c|c|c|}
\hline & \multicolumn{3}{|c|}{ Before Deletion } & \multicolumn{3}{|c|}{ After Deletion } & \multirow{2}{*}{$\begin{array}{c}\text { Chsq } \\
2 \text { DoF }\end{array}$} & \multicolumn{2}{|c|}{ Difference (After minus } & \multirow{2}{*}{$\begin{array}{l}\text { Before) } \\
\text { sig. inc. }\end{array}$} \\
\hline & -ive & ntrl & $+\mathrm{ve}$ & -ive & ntrl & $+\mathrm{ve}$ & & sig. dec. & no sig. change & \\
\hline$\alpha_{0}$ & 12 & 687 & 29 & 13 & 683 & 32 & 0.905 & 6 & 716 & 6 \\
\hline$\alpha_{1}$ & 501 & 226 & 1 & 510 & 217 & 1 & 0.877 & 56 & 587 & 85 \\
\hline$\alpha_{2}$ & 313 & 413 & 2 & 341 & 384 & 3 & 0.293 & 30 & 662 & 36 \\
\hline$\alpha_{3}$ & 169 & 549 & 10 & 174 & 546 & 8 & 0.859 & 19 & 691 & 18 \\
\hline$\alpha_{4}$ & 86 & 625 & 17 & 93 & 624 & 11 & 0.458 & 9 & 707 & 12 \\
\hline$\alpha_{5}$ & 48 & 669 & 11 & 43 & 671 & 14 & 0.727 & 8 & 712 & 8 \\
\hline$\beta_{0}$ & 0 & 184 & 544 & 1 & 189 & 538 & 0.577 & 95 & 564 & 69 \\
\hline$\beta_{1}$ & 4 & 365 & 359 & 7 & 357 & 364 & 0.625 & 71 & 602 & 55 \\
\hline$\beta_{2}$ & 3 & 552 & 173 & 11 & 541 & 176 & 0.095 & 35 & 666 & 27 \\
\hline$\beta_{3}$ & 13 & 624 & 91 & 14 & 620 & 94 & 0.952 & 18 & 693 & 17 \\
\hline$\beta_{4}$ & 16 & 671 & 41 & 12 & 669 & 47 & 0.612 & 21 & 696 & 11 \\
\hline$\beta_{5}$ & 15 & 683 & 30 & 14 & 683 & 31 & 0.975 & 12 & 711 & 5 \\
\hline$\gamma_{0}$ & 79 & 551 & 98 & 48 & 569 & 111 & 0.013 & 22 & 680 & 26 \\
\hline$\gamma_{1}$ & 91 & 470 & 167 & 104 & 483 & 141 & 0.198 & 42 & 615 & 71 \\
\hline$\gamma_{2}$ & 31 & 625 & 72 & 41 & 626 & 61 & 0.317 & 17 & 686 & 25 \\
\hline$\gamma_{3}$ & 17 & 671 & 40 & 27 & 662 & 39 & 0.309 & 5 & 702 & 21 \\
\hline$\gamma_{4}$ & 11 & 689 & 28 & 18 & 680 & 30 & 0.403 & 7 & 708 & 13 \\
\hline$\gamma_{5}$ & 10 & 695 & 23 & 10 & 691 & 27 & 0.847 & 7 & 711 & 10 \\
\hline$\delta_{1}$ & 4 & 170 & 554 & 2 & 170 & 556 & 0.715 & 110 & 507 & 111 \\
\hline$\delta_{2}$ & 16 & 341 & 371 & 6 & 329 & 393 & 0.067 & 58 & 613 & 57 \\
\hline$\delta_{3}$ & 9 & 482 & 237 & 6 & 471 & 251 & 0.569 & 39 & 651 & 38 \\
\hline$\delta_{4}$ & 13 & 537 & 178 & 13 & 520 & 195 & 0.592 & 27 & 664 & 37 \\
\hline$\delta_{5}$ & 10 & 553 & 165 & 15 & 559 & 154 & 0.494 & 24 & 678 & 26 \\
\hline$A$ & 492 & 230 & 6 & 492 & 232 & 4 & 0.815 & 54 & 598 & 76 \\
\hline$B$ & 0 & 178 & 550 & 1 & 184 & 543 & 0.564 & 93 & 575 & 60 \\
\hline$\Gamma$ & 44 & 576 & 108 & 57 & 567 & 104 & 0.403 & 29 & 649 & 50 \\
\hline$\Delta$ & 2 & 92 & 634 & 1 & 117 & 610 & 0.151 & 90 & 511 & 127 \\
\hline
\end{tabular}




\section{Table C.5: Difference in Frequency of Changes in Model Parameters for Addition Events (OLS vs. WLS)}

The table reports the difference in frequency of changes in model parameters between OLS and WLS for the addition events. The $1 \%$ is defined as the significance level. Bold format denotes the significant difference including increase and decrease in the magnitude of parameters which is greater than or equal to $10 \%$ of the sample size, 1,127 .

\begin{tabular}{cccccccccc}
\hline \multicolumn{1}{c}{ Before Addition } & \multicolumn{3}{c}{ After Addition } & \multicolumn{3}{c}{ Difference (After minus Before) } \\
& - -ive & ntrl & sig. dec. & sig. dec. & sig. dec. & +ve & sig. dec. & no sig. change & sig. inc. \\
\hline$\alpha_{0}$ & -54 & 24 & 30 & -57 & 47 & 10 & -1 & -1 & 2 \\
$\alpha_{1}$ & -86 & 86 & 0 & -109 & 110 & -1 & -40 & 74 & -34 \\
$\alpha_{2}$ & -224 & 224 & 0 & -246 & 246 & 0 & -23 & 43 & -20 \\
$\alpha_{3}$ & -247 & 250 & -3 & -281 & 273 & 8 & -13 & 26 & -13 \\
$\alpha_{4}$ & -198 & 197 & 1 & -205 & 201 & 4 & -8 & 21 & -13 \\
$\alpha_{5}$ & -144 & 148 & -4 & -134 & 136 & -2 & -4 & 5 & -1 \\
$\beta_{0}$ & 1 & 113 & -114 & 1 & 143 & -144 & -78 & $\mathbf{1 6 5}$ & -87 \\
$\beta_{1}$ & 1 & 285 & -286 & 1 & 280 & -281 & -52 & $\mathbf{1 4 5}$ & -93 \\
$\beta_{2}$ & -1 & 334 & -333 & 3 & 297 & -300 & -21 & 61 & -40 \\
$\beta_{3}$ & 0 & 251 & -251 & -5 & 204 & -199 & -14 & 56 & -42 \\
$\beta_{4}$ & 1 & 147 & -148 & 2 & 157 & -159 & -7 & 25 & -18 \\
$\beta_{5}$ & -2 & 81 & -79 & -5 & 91 & -86 & -5 & 14 & -9 \\
$\gamma_{0}$ & -80 & -48 & 128 & -54 & -55 & 109 & 36 & -37 & 1 \\
$\gamma_{1}$ & -114 & -66 & 180 & -125 & -45 & 170 & -3 & 12 & -9 \\
$\gamma_{2}$ & -88 & 23 & 65 & -84 & 44 & 40 & 0 & 6 & -6 \\
$\gamma_{3}$ & -51 & 32 & 19 & -70 & 60 & 10 & 1 & 1 & -2 \\
$\gamma_{4}$ & -40 & 30 & 10 & -30 & 33 & -3 & 1 & -4 & 3 \\
$\gamma_{5}$ & -6 & 16 & -10 & -15 & 17 & -2 & -2 & 4 & -2 \\
$\delta_{1}$ & -1 & 155 & -154 & -1 & 154 & -153 & -12 & 11 & 1 \\
$\delta_{2}$ & 1 & 198 & -199 & -1 & 183 & -182 & -2 & 5 & -3 \\
$\delta_{3}$ & 3 & 157 & -160 & 0 & 152 & -152 & -3 & 2 & 1 \\
$\delta_{4}$ & 1 & 160 & -161 & 0 & 128 & -128 & -2 & 0 & 2 \\
$\delta_{5}$ & 2 & 109 & -111 & 1 & 88 & -89 & 4 & -5 & 1 \\
$A$ & -175 & 175 & 0 & -241 & 240 & 1 & -36 & 79 & -43 \\
$B$ & -1 & 61 & -60 & -1 & 88 & -87 & -76 & $\mathbf{1 7 6}$ & -100 \\
$\Gamma$ & -105 & -33 & 138 & -110 & -26 & 136 & 15 & -11 & -4 \\
$\Delta$ & 0 & 60 & -60 & -1 & 69 & -68 & -4 & 11 & -7 \\
\hline
\end{tabular}




\section{Table C.6: Difference in Frequency of Changes in Model Parameters for Deletion Events (OLS vs. WLS)}

The table reports the difference in frequency of changes in model parameters between OLS and WLS for the deletion events. The $1 \%$ is defined as the significance level. Bold format denotes the significant difference including increase and decrease in the magnitude of parameters which is greater than or equal to $10 \%$ of the sample size, 728 .

\begin{tabular}{|c|c|c|c|c|c|c|c|c|c|}
\hline & $\mathrm{Be}$ & ore $\mathrm{D}$ & eletion & Aft & Deletio & & Differer & e (After minus & Before) \\
\hline & -ive & ntrl & sig. dec. & sig. dec. & sig. dec. & $+\mathrm{ve}$ & sig. dec. & no sig. change & sig. inc. \\
\hline$\alpha_{0}$ & -17 & 1 & 16 & -10 & -4 & 14 & 4 & -7 & 3 \\
\hline$\alpha_{1}$ & -122 & 122 & 0 & -141 & 141 & 0 & -19 & 60 & -41 \\
\hline$\alpha_{2}$ & -186 & 184 & 2 & -206 & 206 & 0 & -6 & 34 & -28 \\
\hline$\alpha_{3}$ & -195 & 193 & 2 & -201 & 203 & -2 & -8 & 24 & -16 \\
\hline$\alpha_{4}$ & -101 & 98 & 3 & -142 & 139 & 3 & -5 & 16 & -11 \\
\hline$\alpha_{5}$ & -50 & 47 & 3 & -75 & 75 & 0 & -1 & 4 & -3 \\
\hline$\beta_{0}$ & 0 & 124 & -124 & 1 & 123 & -124 & -83 & 118 & -35 \\
\hline$\beta_{1}$ & 1 & 193 & -194 & 1 & 196 & -197 & -37 & 58 & -21 \\
\hline$\beta_{2}$ & 2 & 145 & -147 & -1 & 161 & -160 & -19 & 32 & -13 \\
\hline$\beta_{3}$ & 0 & 80 & -80 & 0 & 100 & -100 & -5 & 8 & -3 \\
\hline$\beta_{4}$ & 2 & 37 & -39 & 1 & 36 & -37 & -3 & 4 & -1 \\
\hline$\beta_{5}$ & 0 & 14 & -14 & 1 & 13 & -14 & 0 & 1 & -1 \\
\hline$\gamma_{0}$ & -35 & -8 & 43 & -23 & -27 & 50 & 6 & -11 & 5 \\
\hline$\gamma_{1}$ & -110 & 26 & 84 & -134 & 55 & 79 & -3 & 21 & -18 \\
\hline$\gamma_{2}$ & -52 & 29 & 23 & -65 & 45 & 20 & -2 & 10 & -8 \\
\hline$\gamma_{3}$ & -40 & 34 & 6 & -43 & 33 & 10 & 0 & 6 & -6 \\
\hline$\gamma_{4}$ & -17 & 11 & 6 & -18 & 14 & 4 & 0 & -1 & 1 \\
\hline$\gamma_{5}$ & -5 & 3 & 2 & -4 & 0 & 4 & 0 & -2 & 2 \\
\hline$\delta_{1}$ & 1 & 98 & -99 & 0 & 123 & -123 & 3 & -8 & 5 \\
\hline$\delta_{2}$ & 3 & 105 & -108 & 3 & 101 & -104 & 3 & 2 & -5 \\
\hline$\delta_{3}$ & -1 & 73 & -72 & 0 & 94 & -94 & 0 & 1 & -1 \\
\hline$\delta_{4}$ & 0 & 52 & -52 & -1 & 50 & -49 & 0 & -3 & 3 \\
\hline$\delta_{5}$ & -1 & 40 & -39 & 0 & 20 & -20 & -2 & 3 & -1 \\
\hline$A$ & -214 & 214 & 0 & -209 & 209 & 0 & -13 & 47 & -34 \\
\hline$B$ & 0 & 120 & -120 & 2 & 76 & -78 & -61 & 90 & -29 \\
\hline$\Gamma$ & -85 & 23 & 62 & -96 & 41 & 55 & -5 & 4 & 1 \\
\hline$\Delta$ & 0 & 73 & -73 & 0 & 79 & -79 & 11 & -12 & 1 \\
\hline
\end{tabular}


Table C.7: Difference in Frequency of Changes in Model Parameters for Addition Events (VAR vs. WVAR)

The table reports the difference in frequency of changes in model parameters between VAR and WVAR for the addition events. The $1 \%$ is defined as the significance level. Bold format denotes the significant difference including increase and decrease in the magnitude of parameters which is greater than or equal to $10 \%$ of the sample size, 1,127 .

\begin{tabular}{|c|c|c|c|c|c|c|c|c|c|}
\hline & \multicolumn{3}{|c|}{ Before Addition } & \multicolumn{3}{|c|}{ After Addition } & \multicolumn{2}{|c|}{ Difference (After minus } & \multirow{2}{*}{$\begin{array}{l}\text { Before) } \\
\text { sig. inc. }\end{array}$} \\
\hline & -ive & ntrl & sig. dec. & sig. dec. & sig. dec. & $+\mathrm{ve}$ & sig. dec. & no sig. change & \\
\hline$\alpha_{0}$ & -50 & 29 & 21 & -46 & 19 & 27 & -1 & 2 & -1 \\
\hline$\alpha_{1}$ & -145 & 144 & 1 & -160 & 158 & 2 & -58 & 88 & -30 \\
\hline$\alpha_{2}$ & -224 & 218 & 6 & -230 & 227 & 3 & -33 & 68 & -35 \\
\hline$\alpha_{3}$ & -267 & 258 & 9 & -313 & 295 & 18 & -29 & 53 & -24 \\
\hline$\alpha_{4}$ & -257 & 241 & 16 & -230 & 214 & 16 & -15 & 31 & -16 \\
\hline$\alpha_{5}$ & -200 & 182 & 18 & -179 & 169 & 10 & -5 & 9 & -4 \\
\hline$\beta_{0}$ & 0 & 97 & -97 & 0 & 140 & -140 & -83 & 174 & -91 \\
\hline$\beta_{1}$ & 1 & 117 & -118 & 4 & 136 & -140 & -13 & 41 & -28 \\
\hline$\beta_{2}$ & 6 & 156 & -162 & 8 & 123 & -131 & 3 & -11 & 8 \\
\hline$\beta_{3}$ & 5 & 94 & -99 & 4 & 95 & -99 & -6 & 9 & -3 \\
\hline$\beta_{4}$ & 8 & 49 & -57 & 9 & 58 & -67 & 5 & -2 & -3 \\
\hline$\beta_{5}$ & 5 & -7 & 2 & 5 & 14 & -19 & 8 & -29 & 21 \\
\hline$\gamma_{0}$ & -93 & -41 & 134 & -75 & -25 & 100 & 41 & -34 & -7 \\
\hline$\gamma_{1}$ & -76 & -174 & 250 & -67 & -177 & 244 & 72 & -103 & 31 \\
\hline$\gamma_{2}$ & -37 & -85 & 122 & -48 & -65 & 113 & 44 & -64 & 20 \\
\hline$\gamma_{3}$ & -29 & -61 & 90 & -51 & -17 & 68 & 39 & -50 & 11 \\
\hline$\gamma_{4}$ & -22 & -48 & 70 & -17 & -21 & 38 & 32 & -47 & 15 \\
\hline$\gamma_{5}$ & 2 & -53 & 51 & 1 & -40 & 39 & 31 & -34 & 3 \\
\hline$\delta_{1}$ & -1 & -41 & 42 & 4 & -45 & 41 & 123 & -220 & 97 \\
\hline$\delta_{2}$ & 3 & 84 & -87 & 14 & 44 & -58 & 40 & -115 & 75 \\
\hline$\delta_{3}$ & 6 & 65 & -71 & 9 & 40 & -49 & 33 & -82 & 49 \\
\hline$\delta_{4}$ & -5 & 50 & -45 & 4 & 27 & -31 & 23 & -66 & 43 \\
\hline$\delta_{5}$ & 6 & 12 & -18 & 7 & 21 & -28 & 34 & -71 & 37 \\
\hline$A$ & -138 & 136 & 2 & -146 & 141 & 5 & -27 & 86 & -59 \\
\hline$B$ & 0 & 48 & -48 & 0 & 88 & -88 & -78 & 163 & -85 \\
\hline$\Gamma$ & -76 & -129 & 205 & -72 & -131 & 203 & 77 & -104 & 27 \\
\hline$\Delta$ & 0 & 29 & -29 & 2 & 41 & -43 & 70 & -124 & 54 \\
\hline
\end{tabular}


Table C.8: Difference in Frequency of Changes in Model Parameters for Deletion Events (VAR vs. WVAR)

The table reports the difference in frequency of changes in model parameters between VAR and WVAR for the deletion events. The $1 \%$ is defined as the significance level. Bold format denotes the significant difference including increase and decrease in the magnitude of parameters which is greater than or equal to $10 \%$ of the sample size, 728 .

\begin{tabular}{|c|c|c|c|c|c|c|c|c|c|}
\hline & \multicolumn{3}{|c|}{ Before Deletion } & \multicolumn{3}{|c|}{ After Deletion } & \multicolumn{2}{|c|}{ Difference (After minus } & \multirow{2}{*}{$\begin{array}{l}\text { Before } \\
\text { sig. inc }\end{array}$} \\
\hline & -ive & ntrl & sig. dec. & sig. dec. & sig. dec. & $+\mathrm{ve}$ & sig. dec. & no sig. change & \\
\hline$\alpha_{0}$ & -11 & -6 & 17 & -16 & -6 & 22 & 5 & -11 & 6 \\
\hline$\alpha_{1}$ & -101 & 101 & 0 & -88 & 88 & 0 & -11 & 36 & -25 \\
\hline$\alpha_{2}$ & -149 & 147 & 2 & -145 & 142 & 3 & -17 & 50 & -33 \\
\hline$\alpha_{3}$ & -163 & 157 & 6 & -168 & 162 & 6 & -15 & 29 & -14 \\
\hline$\alpha_{4}$ & -98 & 87 & 11 & -135 & 125 & 10 & -7 & 25 & -18 \\
\hline$\alpha_{5}$ & -76 & 71 & 5 & -85 & 74 & 11 & 1 & 2 & -3 \\
\hline$\beta_{0}$ & 0 & 114 & -114 & 1 & 100 & -101 & -82 & 117 & -35 \\
\hline$\beta_{1}$ & 4 & 95 & -99 & 4 & 88 & -92 & 1 & -5 & 4 \\
\hline$\beta_{2}$ & -2 & 63 & -61 & 8 & 78 & -86 & 0 & -9 & 9 \\
\hline$\beta_{3}$ & 11 & 11 & -22 & 11 & 29 & -40 & 11 & -19 & 8 \\
\hline$\beta_{4}$ & 8 & 4 & -12 & 7 & -5 & -2 & 15 & -21 & 6 \\
\hline$\beta_{5}$ & 8 & -20 & 12 & 5 & -18 & 13 & 10 & -13 & 3 \\
\hline$\gamma_{0}$ & -46 & 9 & 37 & -37 & -4 & 41 & 3 & 1 & -4 \\
\hline$\gamma_{1}$ & -100 & -44 & 144 & -96 & -25 & 121 & 12 & -19 & 7 \\
\hline$\gamma_{2}$ & -42 & -20 & 62 & -44 & -8 & 52 & 11 & -13 & 2 \\
\hline$\gamma_{3}$ & -21 & -9 & 30 & -18 & -19 & 37 & 2 & -16 & 14 \\
\hline$\gamma_{4}$ & -9 & -9 & 18 & -3 & -20 & 23 & 3 & -14 & 11 \\
\hline$\gamma_{5}$ & 2 & -19 & 17 & -1 & -19 & 20 & 3 & -11 & 8 \\
\hline$\delta_{1}$ & 4 & -141 & 137 & 2 & -116 & 114 & 84 & -170 & 86 \\
\hline$\delta_{2}$ & 12 & 36 & -48 & 5 & 38 & -43 & 31 & -59 & 28 \\
\hline$\delta_{3}$ & 4 & 28 & -32 & 4 & 33 & -37 & 12 & -22 & 10 \\
\hline$\delta_{4}$ & 10 & 4 & -14 & 9 & -4 & -5 & 10 & -32 & 22 \\
\hline$\delta_{5}$ & 5 & -15 & 10 & 10 & -8 & -2 & 13 & -24 & 11 \\
\hline$A$ & -166 & 161 & 5 & -166 & 162 & 4 & -21 & 73 & -52 \\
\hline$B$ & 0 & 113 & -113 & 1 & 88 & -89 & -66 & 98 & -32 \\
\hline$\Gamma$ & -72 & -14 & 86 & -68 & -18 & 86 & 13 & -34 & 21 \\
\hline$\Delta$ & 2 & 26 & -28 & 1 & 35 & -36 & 24 & -63 & 39 \\
\hline
\end{tabular}




\section{Table C.9: Difference in Frequency of Changes in Model Parameters for Addition Events (OLS vs. VAR)}

The table reports the difference in frequency of changes in model parameters between OLS and VAR for the addition events. The $1 \%$ is defined as the significance level. Bold format denotes the significant difference including increase and decrease in the magnitude of parameters which is greater than or equal to $10 \%$ of the sample size, 1,127 .

\begin{tabular}{cccccccccc}
\hline \multicolumn{4}{c}{ Before Addition } & \multicolumn{3}{c}{ After Addition } & \multicolumn{3}{c}{ Difference (After minus Before) } \\
& - -ive & ntrl & sig. dec. & sig. dec. & sig. dec. & +ve & sig. dec. & no sig. change & sig. inc. \\
\hline$\alpha_{0}$ & -8 & 17 & -9 & -8 & 22 & -14 & -1 & -2 & 3 \\
$\alpha_{1}$ & -7 & 6 & 1 & -3 & 4 & -1 & 86 & $\mathbf{- 1 3 4}$ & 48 \\
$\alpha_{2}$ & 20 & -20 & 0 & 58 & -59 & 1 & 62 & $\mathbf{- 1 1 4}$ & 52 \\
$\alpha_{3}$ & 73 & -68 & -5 & 79 & -88 & 9 & 48 & -82 & 34 \\
$\alpha_{4}$ & 71 & -77 & 6 & 66 & -76 & 10 & 31 & -59 & 28 \\
$\alpha_{5}$ & 69 & -75 & 6 & 53 & -63 & 10 & 11 & -29 & 18 \\
$\beta_{0}$ & -1 & -11 & 12 & 1 & -18 & 17 & 3 & -18 & 15 \\
$\beta_{1}$ & 1 & -41 & 40 & 0 & -52 & 52 & 54 & $\mathbf{- 1 3 5}$ & 81 \\
$\beta_{2}$ & 0 & -5 & 5 & 0 & -13 & 13 & 15 & -62 & 47 \\
$\beta_{3}$ & 0 & 4 & -4 & 2 & -26 & 24 & 15 & -41 & 26 \\
$\beta_{4}$ & 1 & -24 & 23 & 2 & -15 & 13 & 4 & -20 & 16 \\
$\beta_{5}$ & 0 & -13 & 13 & 11 & -33 & 22 & 1 & -16 & 15 \\
$\gamma_{0}$ & 12 & -6 & -6 & 22 & -19 & -3 & -7 & 2 & 5 \\
$\gamma_{1}$ & 9 & -14 & 5 & -21 & 8 & 13 & 7 & -13 & 6 \\
$\gamma_{2}$ & -15 & 4 & 11 & -9 & 7 & 2 & 13 & -16 & 3 \\
$\gamma_{3}$ & -8 & 7 & 1 & -17 & 17 & 0 & 4 & -4 & 0 \\
$\gamma_{4}$ & -8 & 4 & 4 & -2 & -12 & 14 & 3 & -6 & 3 \\
$\gamma_{5}$ & -4 & 2 & 2 & -11 & 11 & 0 & 4 & -6 & 2 \\
$\delta_{1}$ & 0 & -4 & 4 & -1 & -14 & 15 & 71 & $\mathbf{- 1 2 6}$ & 55 \\
$\delta_{2}$ & 3 & -133 & 130 & 0 & -136 & 136 & 78 & $\mathbf{- 1 2 3}$ & 45 \\
$\delta_{3}$ & 1 & -156 & 155 & 0 & -160 & 160 & 49 & -83 & 34 \\
$\delta_{4}$ & 10 & -137 & 127 & 3 & -128 & 125 & 48 & -88 & 40 \\
$\delta_{5}$ & 5 & -126 & 121 & 5 & -130 & 125 & 29 & -65 & 36 \\
$A$ & 46 & -45 & -1 & 54 & -55 & 1 & 85 & $\mathbf{- 1 7 2}$ & 87 \\
$B$ & -2 & 8 & -6 & -1 & 0 & 1 & 3 & 8 & -11 \\
$\Gamma$ & -6 & 4 & 2 & -15 & 16 & -1 & 13 & -16 & 3 \\
$\Delta$ & 0 & -34 & 34 & -1 & -35 & 36 & 117 & $\mathbf{- 1 9 6}$ & 79 \\
\hline & & & & & & & & & \\
\end{tabular}




\section{Table C.10: Difference in Frequency of Changes in Model Parameters for Deletion Events (OLS vs. VAR)}

The table reports the difference in frequency of changes in model parameters between OLS and VAR for the deletion events. The $1 \%$ is defined as the significance level. Bold format denotes the significant difference including increase and decrease in the magnitude of parameters which is greater than or equal to $10 \%$ of the sample size, 728 .

\begin{tabular}{|c|c|c|c|c|c|c|c|c|c|}
\hline & \multicolumn{3}{|c|}{ Before Deletion } & \multicolumn{3}{|c|}{ After Deletion } & \multicolumn{2}{|c|}{ Difference (After minus } & \multirow{2}{*}{$\begin{array}{l}\text { Before) } \\
\text { sig. inc. }\end{array}$} \\
\hline & -ive & ntrl & sig. dec. & sig. dec. & sig. dec. & $+\mathrm{ve}$ & sig. dec. & no sig. change & \\
\hline$\alpha_{0}$ & -7 & 12 & -5 & 3 & 4 & -7 & -2 & 4 & -2 \\
\hline$\alpha_{1}$ & -46 & 45 & 1 & -59 & 58 & 1 & 28 & -62 & 34 \\
\hline$\alpha_{2}$ & -9 & 9 & 0 & -18 & 18 & 0 & 30 & -58 & 28 \\
\hline$\alpha_{3}$ & 20 & -23 & 3 & 0 & 1 & -1 & 23 & -36 & 13 \\
\hline$\alpha_{4}$ & 25 & -30 & 5 & 12 & -13 & 1 & 9 & -26 & 17 \\
\hline$\alpha_{5}$ & 44 & -49 & 5 & 20 & -21 & 1 & 4 & -10 & 6 \\
\hline$\beta_{0}$ & 0 & -10 & 10 & 0 & -8 & 8 & 9 & -16 & 7 \\
\hline$\beta_{1}$ & 0 & -43 & 43 & 1 & -22 & 21 & 16 & -35 & 19 \\
\hline$\beta_{2}$ & 4 & -9 & 5 & -1 & -5 & 6 & 14 & -15 & 1 \\
\hline$\beta_{3}$ & -1 & 6 & -5 & 0 & 6 & -6 & 0 & -4 & 4 \\
\hline$\beta_{4}$ & 6 & -11 & 5 & 2 & 5 & -7 & 1 & -2 & 1 \\
\hline$\beta_{5}$ & 0 & 5 & -5 & 2 & 5 & -7 & 0 & 2 & -2 \\
\hline$\gamma_{0}$ & 17 & -13 & -4 & 17 & -16 & -1 & 0 & -3 & 3 \\
\hline$\gamma_{1}$ & 5 & -3 & -2 & -15 & 15 & 0 & 8 & -17 & 9 \\
\hline$\gamma_{2}$ & 1 & -3 & 2 & -15 & 12 & 3 & 0 & -6 & 6 \\
\hline$\gamma_{3}$ & -13 & 9 & 4 & -14 & 19 & -5 & 1 & 1 & -2 \\
\hline$\gamma_{4}$ & -3 & -2 & 5 & -7 & 6 & 1 & 3 & -4 & 1 \\
\hline$\gamma_{5}$ & -1 & -1 & 2 & 3 & -4 & 1 & 4 & -4 & 0 \\
\hline$\delta_{1}$ & 0 & 87 & -87 & -1 & 79 & -78 & 10 & -12 & 2 \\
\hline$\delta_{2}$ & 3 & -107 & 104 & 0 & -108 & 108 & 24 & -45 & 21 \\
\hline$\delta_{3}$ & 3 & -86 & 83 & 1 & -68 & 67 & 26 & -43 & 17 \\
\hline$\delta_{4}$ & 2 & -72 & 70 & 0 & -67 & 67 & 16 & -29 & 13 \\
\hline$\delta_{5}$ & 4 & -59 & 55 & 3 & -65 & 62 & 8 & -18 & 10 \\
\hline$A$ & 64 & -65 & 1 & 51 & -51 & 0 & 49 & -119 & 70 \\
\hline$B$ & 0 & 1 & -1 & 0 & -15 & 15 & 1 & -2 & 1 \\
\hline$\Gamma$ & -14 & 11 & 3 & -16 & 13 & 3 & 0 & 2 & -2 \\
\hline$\Delta$ & 0 & -49 & 49 & -1 & -47 & 48 & 48 & -104 & 56 \\
\hline
\end{tabular}




\section{Table C.11: Difference in Frequency of Changes in Model Parameters for Addition Events (WLS vs. WVAR)}

The table reports the difference in frequency of changes in model parameters between WLS and WVAR for the addition events. The $1 \%$ is defined as the significance level. Bold format denotes the significant difference including increase and decrease in the magnitude of parameters which is greater than or equal to $10 \%$ of the sample size, 1,127 .

\begin{tabular}{cccccccccc}
\hline & \multicolumn{3}{c}{ Before Addition } & \multicolumn{3}{c}{ After Addition } & \multicolumn{3}{c}{ Difference (After minus Before) } \\
& - ive & ntrl & sig. dec. & sig. dec. & sig. dec. & + +ve & sig. dec. & no sig. change & sig. inc. \\
\hline$\alpha_{0}$ & -4 & 22 & -18 & 3 & -6 & 3 & -1 & 1 & 0 \\
$\alpha_{1}$ & -66 & 64 & 2 & -54 & 52 & 2 & 68 & $\mathbf{- 1 2 0}$ & 52 \\
$\alpha_{2}$ & 20 & -26 & 6 & 74 & -78 & 4 & 52 & -89 & 37 \\
$\alpha_{3}$ & 53 & -60 & 7 & 47 & -66 & 19 & 32 & -55 & 23 \\
$\alpha_{4}$ & 12 & -33 & 21 & 41 & -63 & 22 & 24 & -49 & 25 \\
$\alpha_{5}$ & 13 & -41 & 28 & 8 & -30 & 22 & 10 & -25 & 15 \\
$\beta_{0}$ & -2 & -27 & 29 & 0 & -21 & 21 & -2 & -9 & 11 \\
$\beta_{1}$ & 1 & -209 & 208 & 3 & -196 & 193 & 93 & $\mathbf{- 2 3 9}$ & 146 \\
$\beta_{2}$ & 7 & -183 & 176 & 5 & -187 & 182 & 39 & $\mathbf{- 1 3 4}$ & 95 \\
$\beta_{3}$ & 5 & -153 & 148 & 11 & -135 & 124 & 23 & -88 & 65 \\
$\beta_{4}$ & 8 & -122 & 114 & 9 & -114 & 105 & 16 & -47 & 31 \\
$\beta_{5}$ & 7 & -101 & 94 & 21 & -110 & 89 & 14 & -59 & 45 \\
$\gamma_{0}$ & -1 & 1 & 0 & 1 & 11 & -12 & -2 & 5 & -3 \\
$\gamma_{1}$ & 47 & -122 & 75 & 37 & -124 & 87 & 82 & $\mathbf{- 1 2 8}$ & 46 \\
$\gamma_{2}$ & 36 & -104 & 68 & 27 & -102 & 75 & 57 & -86 & 29 \\
$\gamma_{3}$ & 14 & -86 & 72 & 2 & -60 & 58 & 42 & -55 & 13 \\
$\gamma_{4}$ & 10 & -74 & 64 & 11 & -66 & 55 & 34 & -49 & 15 \\
$\gamma_{5}$ & 4 & -67 & 63 & 5 & -46 & 41 & 37 & -44 & 7 \\
$\delta_{1}$ & 0 & -200 & 200 & 4 & -213 & 209 & 206 & $\mathbf{- 3 5 7}$ & 151 \\
$\delta_{2}$ & 5 & -247 & 242 & 15 & -275 & 260 & 120 & $\mathbf{- 2 4 3}$ & 123 \\
$\delta_{3}$ & 4 & -248 & 244 & 9 & -272 & 263 & 85 & $\mathbf{- 1 6 7}$ & 82 \\
$\delta_{4}$ & 4 & -247 & 243 & 7 & -229 & 222 & 73 & $\mathbf{- 1 5 4}$ & 81 \\
$\delta_{5}$ & 9 & -223 & 214 & 11 & -197 & 186 & 59 & $\mathbf{- 1 3 1}$ & 72 \\
$A$ & 83 & -84 & 1 & 149 & -154 & 5 & 94 & $\mathbf{- 1 6 5}$ & 71 \\
$B$ & -1 & -5 & 6 & 0 & 0 & 0 & 1 & -5 & 4 \\
$\Gamma$ & 23 & -92 & 69 & 23 & -89 & 66 & 75 & $\mathbf{- 1 0 9}$ & 34 \\
$\Delta$ & 0 & -65 & 65 & 2 & -63 & 61 & 191 & $\mathbf{- 3 3 1}$ & 140 \\
\hline & & & & & & & & & \\
\end{tabular}


Table C.12: Difference in Frequency of Changes in Model Parameters for Deletion Events (WLS vs. WVAR)

The table reports the difference in frequency of changes in model parameters between WLS and WVAR for the deletion events. The $1 \%$ is defined as the significance level. Bold format denotes the significant difference including increase and decrease in the magnitude of parameters which is greater than or equal to $10 \%$ of the sample size, 728 .

\begin{tabular}{|c|c|c|c|c|c|c|c|c|c|}
\hline & \multicolumn{3}{|c|}{ Before Deletion } & \multicolumn{3}{|c|}{ After Deletion } & \multicolumn{2}{|c|}{ Difference (After minus } & \multirow{2}{*}{$\begin{array}{l}\text { Before) } \\
\text { sig. inc. }\end{array}$} \\
\hline & -ive & ntrl & sig. dec. & sig. dec. & sig. dec. & $+\mathrm{ve}$ & sig. dec. & no sig. change & \\
\hline$\alpha_{0}$ & -1 & 5 & -4 & -3 & 2 & 1 & -1 & 0 & 1 \\
\hline$\alpha_{1}$ & -25 & 24 & 1 & -6 & 5 & 1 & 36 & -86 & 50 \\
\hline$\alpha_{2}$ & 28 & -28 & 0 & 43 & -46 & 3 & 19 & -42 & 23 \\
\hline$\alpha_{3}$ & 52 & -59 & 7 & 33 & -40 & 7 & 16 & -31 & 15 \\
\hline$\alpha_{4}$ & 28 & -41 & 13 & 19 & -27 & 8 & 7 & -17 & 10 \\
\hline$\alpha_{5}$ & 18 & -25 & 7 & 10 & -22 & 12 & 6 & -12 & 6 \\
\hline$\beta_{0}$ & 0 & -20 & 20 & 0 & -31 & 31 & 10 & -17 & 7 \\
\hline$\beta_{1}$ & 3 & -141 & 138 & 4 & -130 & 126 & 54 & -98 & 44 \\
\hline$\beta_{2}$ & 0 & -91 & 91 & 8 & -88 & 80 & 33 & -56 & 23 \\
\hline$\beta_{3}$ & 10 & -63 & 53 & 11 & -65 & 54 & 16 & -31 & 15 \\
\hline$\beta_{4}$ & 12 & -44 & 32 & 8 & -36 & 28 & 19 & -27 & 8 \\
\hline$\beta_{5}$ & 8 & -29 & 21 & 6 & -26 & 20 & 10 & -12 & 2 \\
\hline$\gamma_{0}$ & 6 & 4 & -10 & 3 & 7 & -10 & -3 & 9 & -6 \\
\hline$\gamma_{1}$ & 15 & -73 & 58 & 23 & -65 & 42 & 23 & -57 & 34 \\
\hline$\gamma_{2}$ & 11 & -52 & 41 & 6 & -41 & 35 & 13 & -29 & 16 \\
\hline$\gamma_{3}$ & 6 & -34 & 28 & 11 & -33 & 22 & 3 & -21 & 18 \\
\hline$\gamma_{4}$ & 5 & -22 & 17 & 8 & -28 & 20 & 6 & -17 & 11 \\
\hline$\gamma_{5}$ & 6 & -23 & 17 & 6 & -23 & 17 & 7 & -13 & 6 \\
\hline$\delta_{1}$ & 3 & -152 & 149 & 1 & -160 & 159 & 91 & -174 & 83 \\
\hline$\delta_{2}$ & 12 & -176 & 164 & 2 & -171 & 169 & 52 & -106 & 54 \\
\hline$\delta_{3}$ & 8 & -131 & 123 & 5 & -129 & 124 & 38 & -66 & 28 \\
\hline$\delta_{4}$ & 12 & -120 & 108 & 10 & -121 & 111 & 26 & -58 & 32 \\
\hline$\delta_{5}$ & 10 & -114 & 104 & 13 & -93 & 80 & 23 & -45 & 22 \\
\hline$A$ & 112 & -118 & 6 & 94 & -98 & 4 & 41 & -93 & 52 \\
\hline$B$ & 0 & -6 & 6 & -1 & -3 & 4 & -4 & 6 & -2 \\
\hline$\Gamma$ & -1 & -26 & 27 & 12 & -46 & 34 & 18 & -36 & 18 \\
\hline$\Delta$ & 2 & -96 & 94 & 0 & -91 & 91 & 61 & -155 & 94 \\
\hline
\end{tabular}




\section{Table C.13: Parameter Dynamics (WLS)}

Based on the model defined at (2.1) using 60 days of trade data by WLS. Each panel shows the results of estimates of the sums of the $\alpha \mathrm{s}, \beta \mathrm{s}, \gamma \mathrm{s}$ and $\delta$ s respectively. Rows of each panel are before the event and columns are after it. "N" and "P" denotes negative and positive respectively. All other changes at a significance level of which is greater than $5 \%$ are classified as neutral. Bold entries in the table are percentages which are substantial. More detailed tables which cover individual parameters are available on request.

\begin{tabular}{|c|c|c|c|c|c|c|c|c|c|c|}
\hline \multicolumn{6}{|c|}{ Addition } & \multicolumn{5}{|c|}{ Deletion } \\
\hline & $\mathrm{N}(1 \%)$ & $\mathrm{N}(5 \%)$ & Neutral & $\mathrm{P}(5 \%)$ & $\mathrm{P}(1 \%)$ & $\mathrm{N}(1 \%)$ & $\mathrm{N}(5 \%)$ & Neutral & $\mathrm{P}(5 \%)$ & $\mathrm{P}(1 \%)$ \\
\hline \multicolumn{11}{|c|}{$A$} \\
\hline $\mathrm{N}(1 \%)$ & 59.54 & 5.94 & 9.76 & 0.00 & 0.00 & 37.23 & 3.57 & 11.26 & 0.14 & 0.00 \\
\hline $\mathrm{N}(5 \%)$ & 3.37 & 1.24 & 3.46 & 0.00 & 0.00 & 6.04 & 2.47 & 6.87 & 0.00 & 0.00 \\
\hline Neutral & 4.97 & 3.11 & 8.34 & 0.09 & 0.09 & 11.26 & 4.40 & 16.35 & 0.14 & 0.00 \\
\hline $\mathrm{P}(5 \%)$ & 0.00 & 0.00 & 0.00 & 0.00 & 0.00 & 0.14 & 0.00 & 0.14 & 0.00 & 0.00 \\
\hline $\mathrm{P}(1 \%)$ & 0.00 & 0.00 & 0.09 & 0.00 & 0.00 & 0.00 & 0.00 & 0.00 & 0.00 & 0.00 \\
\hline \multicolumn{11}{|c|}{$B$} \\
\hline $\mathrm{N}(1 \%)$ & 0.00 & 0.00 & 0.09 & 0.00 & 0.09 & 0.00 & 0.00 & 0.00 & 0.00 & 0.00 \\
\hline $\mathrm{N}(5 \%)$ & 0.00 & 0.00 & 0.00 & 0.00 & 0.00 & 0.00 & 0.00 & 0.00 & 0.14 & 0.14 \\
\hline Neutral & 0.00 & 0.09 & 2.57 & 0.62 & 2.31 & 0.14 & 0.00 & 4.81 & 3.16 & 7.28 \\
\hline $\mathrm{P}(5 \%)$ & 0.00 & 0.00 & 1.06 & 0.98 & 2.40 & 0.00 & 0.00 & 2.47 & 1.37 & 5.77 \\
\hline $\mathrm{P}(1 \%)$ & 0.00 & 0.09 & 4.79 & 3.28 & 81.63 & 0.14 & 0.00 & 9.20 & 4.53 & 60.85 \\
\hline \multicolumn{11}{|c|}{$\Gamma$} \\
\hline $\mathrm{N}(1 \%)$ & 3.11 & 0.35 & 3.64 & 0.00 & 0.09 & 1.79 & 0.41 & 3.98 & 0.00 & 0.00 \\
\hline $\mathrm{N}(5 \%)$ & 0.35 & 0.71 & 3.82 & 0.09 & 0.00 & 0.69 & 1.24 & 4.12 & 0.00 & 0.00 \\
\hline Neutral & 3.02 & 2.66 & 44.54 & 3.11 & 4.88 & 3.57 & 4.81 & 55.91 & 3.30 & 4.81 \\
\hline $\mathrm{P}(5 \%)$ & 0.09 & 0.00 & 4.08 & 0.53 & 1.60 & 0.14 & 0.00 & 3.16 & 0.27 & 0.69 \\
\hline $\mathrm{P}(1 \%)$ & 0.18 & 0.27 & 7.99 & 2.31 & 12.60 & 0.00 & 0.14 & 5.49 & 1.37 & 4.12 \\
\hline \multicolumn{11}{|c|}{$\Delta$} \\
\hline $\mathrm{N}(1 \%)$ & 0.00 & 0.00 & 0.00 & 0.00 & 0.00 & 0.00 & 0.00 & 0.00 & 0.00 & 0.00 \\
\hline $\mathrm{N}(5 \%)$ & 0.00 & 0.00 & 0.00 & 0.00 & 0.09 & 0.00 & 0.00 & 0.14 & 0.00 & 0.00 \\
\hline Neutral & 0.00 & 0.00 & 1.69 & 0.62 & 4.61 & 0.00 & 0.14 & 4.81 & 2.47 & 8.79 \\
\hline $\mathrm{P}(5 \%)$ & 0.00 & 0.00 & 1.06 & 0.18 & 3.46 & 0.00 & 0.00 & 2.88 & 0.96 & 5.63 \\
\hline $\mathrm{P}(1 \%)$ & 0.00 & 0.09 & 6.12 & 4.08 & 77.99 & 0.14 & 0.00 & 10.85 & 6.32 & 56.87 \\
\hline
\end{tabular}




\section{Table C.14: Parameter Dynamics (VAR)}

Based on the model defined at (2.1) using 60 days of trade data by VAR. Each panel shows the results of estimates of the sums of the $\alpha \mathrm{s}, \beta \mathrm{s}, \gamma \mathrm{s}$ and $\delta$ s respectively. Rows of each panel are before the event and columns are after it. "N" and "P" denotes negative and positive respectively. All other changes at a significance level of which is greater than $5 \%$ are classified as neutral. Bold entries in the table are percentages which are substantial. More detailed tables which cover individual parameters are available on request.

\begin{tabular}{|c|c|c|c|c|c|c|c|c|c|c|}
\hline \multicolumn{6}{|c|}{ Addition } & \multicolumn{5}{|c|}{ Deletion } \\
\hline & $\mathrm{N}(1 \%)$ & $\mathrm{N}(5 \%)$ & Neutral & $\mathrm{P}(5 \%)$ & $\mathrm{P}(1 \%)$ & $\mathrm{N}(1 \%)$ & $\mathrm{N}(5 \%)$ & Neutral & $\mathrm{P}(5 \%)$ & $\mathrm{P}(1 \%)$ \\
\hline \multicolumn{11}{|c|}{$A$} \\
\hline $\mathrm{N}(1 \%)$ & 91.13 & 2.22 & 1.42 & 0.00 & 0.09 & 83.52 & 2.88 & 3.98 & 0.00 & 0.00 \\
\hline $\mathrm{N}(5 \%)$ & 1.24 & 0.09 & 0.80 & 0.00 & 0.00 & 3.30 & 0.41 & 0.55 & 0.00 & 0.00 \\
\hline Neutral & 1.69 & 0.09 & 1.24 & 0.00 & 0.00 & 3.43 & 0.41 & 1.37 & 0.00 & 0.00 \\
\hline $\mathrm{P}(5 \%)$ & 0.00 & 0.00 & 0.00 & 0.00 & 0.00 & 0.00 & 0.00 & 0.00 & 0.00 & 0.00 \\
\hline $\mathrm{P}(1 \%)$ & 0.00 & 0.00 & 0.00 & 0.00 & 0.00 & 0.14 & 0.00 & 0.00 & 0.00 & 0.00 \\
\hline \multicolumn{11}{|c|}{$B$} \\
\hline $\mathrm{N}(1 \%)$ & 0.00 & 0.00 & 0.00 & 0.00 & 0.09 & 0.00 & 0.00 & 0.00 & 0.00 & 0.00 \\
\hline $\mathrm{N}(5 \%)$ & 0.00 & 0.00 & 0.00 & 0.00 & 0.00 & 0.00 & 0.00 & 0.00 & 0.00 & 0.00 \\
\hline Neutral & 0.00 & 0.09 & 1.24 & 0.62 & 1.51 & 0.00 & 0.00 & 2.61 & 0.41 & 3.16 \\
\hline $\mathrm{P}(5 \%)$ & 0.00 & 0.00 & 0.71 & 0.09 & 1.06 & 0.00 & 0.00 & 0.82 & 0.55 & 1.37 \\
\hline $\mathrm{P}(1 \%)$ & 0.00 & 0.00 & 1.77 & 1.24 & 91.57 & 0.00 & 0.00 & 5.91 & 2.88 & 82.28 \\
\hline \multicolumn{11}{|c|}{$\Gamma$} \\
\hline $\mathrm{N}(1 \%)$ & 8.16 & 1.60 & 5.77 & 0.18 & 0.27 & 7.42 & 2.20 & 6.32 & 0.00 & 0.00 \\
\hline $\mathrm{N}(5 \%)$ & 1.06 & 0.62 & 3.99 & 0.09 & 0.18 & 2.61 & 1.10 & 4.81 & 0.14 & 0.27 \\
\hline Neutral & 5.32 & 4.53 & 48.09 & 1.86 & 2.48 & 6.87 & 6.59 & 53.16 & 1.79 & 1.37 \\
\hline $\mathrm{P}(5 \%)$ & 0.27 & 0.09 & 3.46 & 0.44 & 0.27 & 0.27 & 0.14 & 1.37 & 0.14 & 0.41 \\
\hline $\mathrm{P}(1 \%)$ & 0.35 & 0.18 & 5.68 & 1.24 & 3.82 & 0.00 & 0.14 & 2.20 & 0.27 & 0.41 \\
\hline \multicolumn{11}{|c|}{$\Delta$} \\
\hline $\mathrm{N}(1 \%)$ & 0.00 & 0.00 & 0.00 & 0.00 & 0.00 & 0.00 & 0.00 & 0.00 & 0.00 & 0.00 \\
\hline $\mathrm{N}(5 \%)$ & 0.00 & 0.00 & 0.09 & 0.00 & 0.00 & 0.00 & 0.00 & 0.00 & 0.00 & 0.00 \\
\hline Neutral & 0.00 & 0.00 & 0.27 & 0.35 & 1.15 & 0.00 & 0.00 & 1.24 & 0.27 & 4.40 \\
\hline $\mathrm{P}(5 \%)$ & 0.00 & 0.00 & 0.35 & 0.09 & 1.06 & 0.00 & 0.00 & 0.27 & 0.14 & 2.75 \\
\hline $\mathrm{P}(1 \%)$ & 0.00 & 0.00 & 2.22 & 1.24 & 93.17 & 0.00 & 0.00 & 6.32 & 3.02 & 81.59 \\
\hline
\end{tabular}




\section{Table C.15: Parameter Dynamics (WVAR)}

Based on the model defined at (2.1) using 60 days of trade data by WVAR. Each panel shows the results of estimates of the sums of the $\alpha \mathrm{s}, \beta \mathrm{s}, \gamma \mathrm{s}$ and $\delta$ s respectively. Rows of each panel are before the event and columns are after it. "N" and "P" denotes negative and positive respectively. All other changes at a significance level of which is greater than $5 \%$ are classified as neutral. Bold entries in the table are percentages which are substantial. More detailed tables which cover individual parameters are available on request.

\begin{tabular}{|c|c|c|c|c|c|c|c|c|c|c|}
\hline \multicolumn{6}{|c|}{ Addition } & \multicolumn{5}{|c|}{ Deletion } \\
\hline & $\mathrm{N}(1 \%)$ & $\mathrm{N}(5 \%)$ & Neutral & $\mathrm{P}(5 \%)$ & $\mathrm{P}(1 \%)$ & $\mathrm{N}(1 \%)$ & $\mathrm{N}(5 \%)$ & Neutral & $\mathrm{P}(5 \%)$ & $\mathrm{P}(1 \%)$ \\
\hline \multicolumn{11}{|c|}{$A$} \\
\hline $\mathrm{N}(1 \%)$ & 71.78 & 3.64 & 6.74 & 0.09 & 0.35 & 53.02 & 4.40 & 9.75 & 0.00 & 0.41 \\
\hline $\mathrm{N}(5 \%)$ & 3.28 & 0.53 & 1.33 & 0.00 & 0.00 & 4.67 & 0.41 & 2.47 & 0.00 & 0.00 \\
\hline Neutral & 5.86 & 0.89 & 4.88 & 0.18 & 0.18 & 9.20 & 2.75 & 11.26 & 0.14 & 0.14 \\
\hline $\mathrm{P}(5 \%)$ & 0.00 & 0.00 & 0.09 & 0.00 & 0.00 & 0.27 & 0.00 & 0.27 & 0.00 & 0.00 \\
\hline $\mathrm{P}(1 \%)$ & 0.18 & 0.00 & 0.00 & 0.00 & 0.00 & 0.41 & 0.14 & 0.27 & 0.00 & 0.00 \\
\hline \multicolumn{11}{|c|}{$B$} \\
\hline $\mathrm{N}(1 \%)$ & 0.00 & 0.00 & 0.00 & 0.00 & 0.09 & 0.00 & 0.00 & 0.00 & 0.00 & 0.00 \\
\hline $\mathrm{N}(5 \%)$ & 0.00 & 0.00 & 0.00 & 0.00 & 0.00 & 0.00 & 0.00 & 0.00 & 0.00 & 0.00 \\
\hline Neutral & 0.00 & 0.00 & 2.75 & 0.62 & 3.02 & 0.00 & 0.00 & 5.77 & 2.88 & 7.83 \\
\hline $\mathrm{P}(5 \%)$ & 0.00 & 0.00 & 0.89 & 0.44 & 1.86 & 0.00 & 0.00 & 1.51 & 1.92 & 4.53 \\
\hline $\mathrm{P}(1 \%)$ & 0.00 & 0.00 & 5.24 & 3.64 & 81.46 & 0.14 & 0.00 & 7.69 & 5.49 & 62.23 \\
\hline \multicolumn{11}{|c|}{$\Gamma$} \\
\hline $\mathrm{N}(1 \%)$ & 3.90 & 0.89 & 4.26 & 0.09 & 0.09 & 2.20 & 0.69 & 3.02 & 0.14 & 0.00 \\
\hline $\mathrm{N}(5 \%)$ & 0.71 & 0.35 & 3.37 & 0.09 & 0.18 & 0.41 & 0.55 & 3.02 & 0.00 & 0.00 \\
\hline Neutral & 3.28 & 1.95 & 37.53 & 2.57 & 5.50 & 4.81 & 4.81 & 52.61 & 2.61 & 5.63 \\
\hline $\mathrm{P}(5 \%)$ & 0.18 & 0.35 & 3.19 & 0.27 & 1.77 & 0.14 & 0.41 & 3.43 & 0.14 & 0.55 \\
\hline $\mathrm{P}(1 \%)$ & 0.71 & 0.00 & 8.87 & 2.40 & 17.48 & 0.27 & 0.00 & 4.95 & 1.51 & 8.10 \\
\hline \multicolumn{11}{|c|}{$\Delta$} \\
\hline $\mathrm{N}(1 \%)$ & 0.00 & 0.00 & 0.00 & 0.00 & 0.00 & 0.00 & 0.00 & 0.14 & 0.00 & 0.14 \\
\hline $\mathrm{N}(5 \%)$ & 0.00 & 0.00 & 0.00 & 0.00 & 0.00 & 0.00 & 0.00 & 0.14 & 0.00 & 0.27 \\
\hline Neutral & 0.00 & 0.00 & 1.06 & 0.18 & 2.66 & 0.00 & 0.00 & 2.20 & 0.41 & 5.63 \\
\hline $\mathrm{P}(5 \%)$ & 0.00 & 0.00 & 0.27 & 0.44 & 1.33 & 0.14 & 0.00 & 1.10 & 0.14 & 2.61 \\
\hline $\mathrm{P}(1 \%)$ & 0.18 & 0.18 & 3.64 & 2.48 & 87.58 & 0.00 & 0.00 & 8.52 & 3.43 & 75.14 \\
\hline
\end{tabular}


Table C.16: P-Values for Tests of Model Parameters (OLS)

Based on the model defined at (2.1) using 60 days of trade data by OLS. The tests for individual parameters and sums are computed as described in Section 2.3.3. The cell entries show the estimated parameters and probabilities (p-val.), respectively. Differences are calculated by absolute value of model parameters between after and before both events. Bold format denotes significance at the $1 \%$ level.

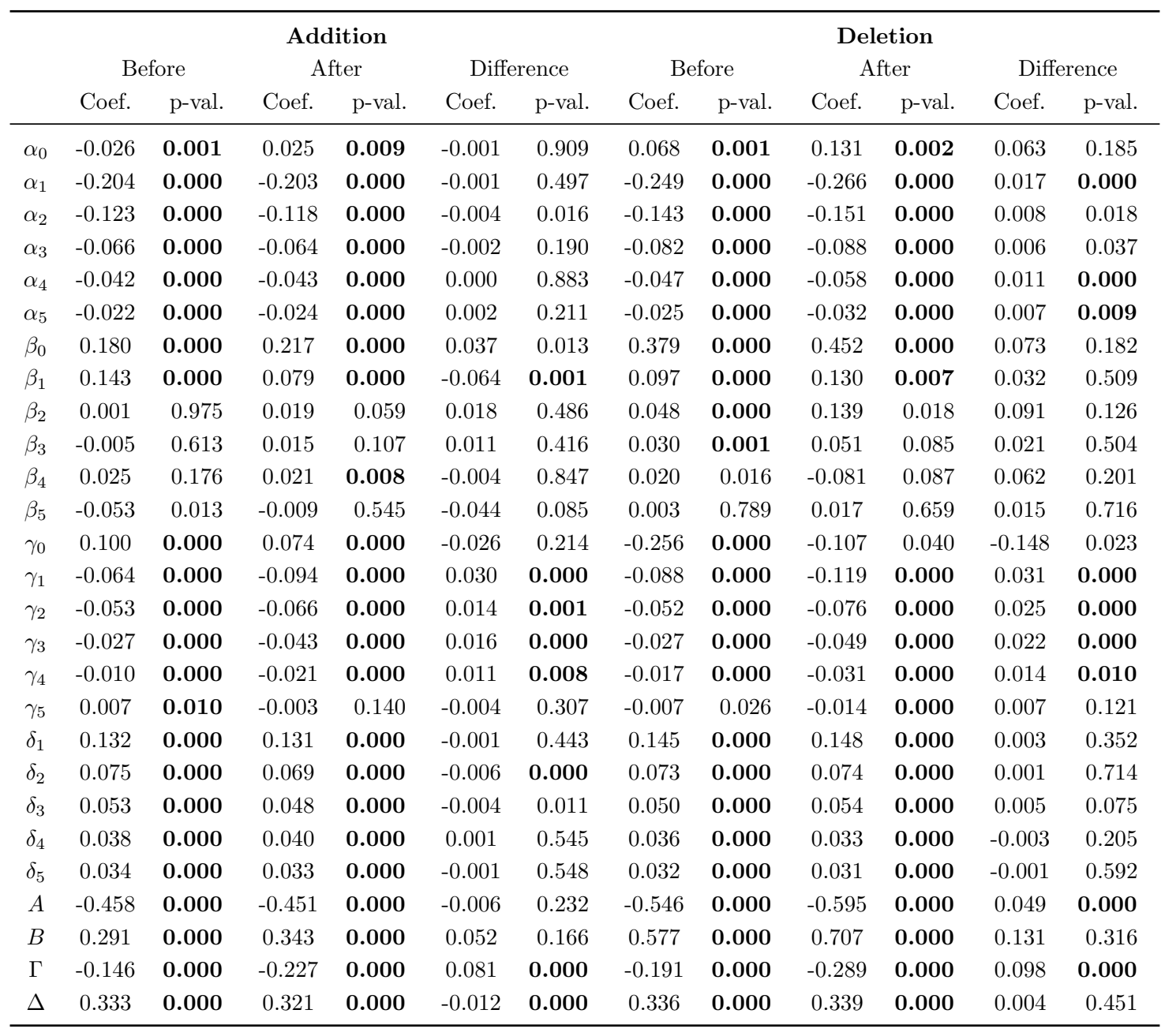




\section{Table C.17: P-Values for Tests of Model Parameters (WLS)}

Based on the model defined at (2.1) using 60 days of trade data by WLS. The tests for individual parameters and sums are computed as described in Section 2.3.3. The cell entries show the estimated parameters and probabilities (p-val.), respectively. Differences are calculated by absolute value of model parameters between after and before both events. Bold format denotes significance at the $1 \%$ level.

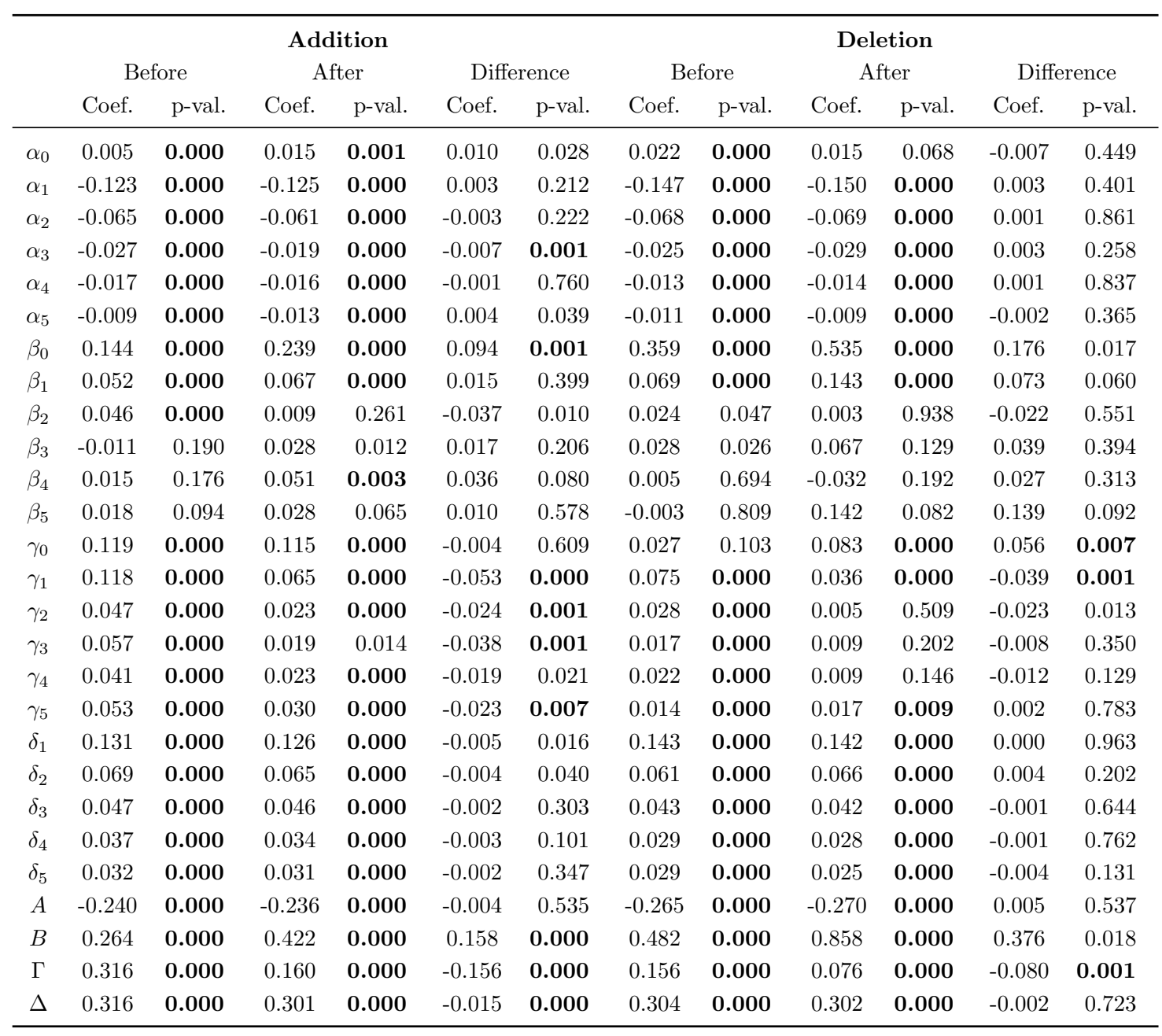




\section{Table C.18: P-Values for Tests of Model Parameters (VAR)}

Based on the model defined at (2.1) using 60 days of trade data by VAR. The tests for individual parameters and sums are computed as described in Section 2.3.3. The cell entries show the estimated parameters and probabilities (p-val.), respectively. Differences are calculated by absolute value of model parameters between after and before both events. Bold format denotes significance at the $1 \%$ level.

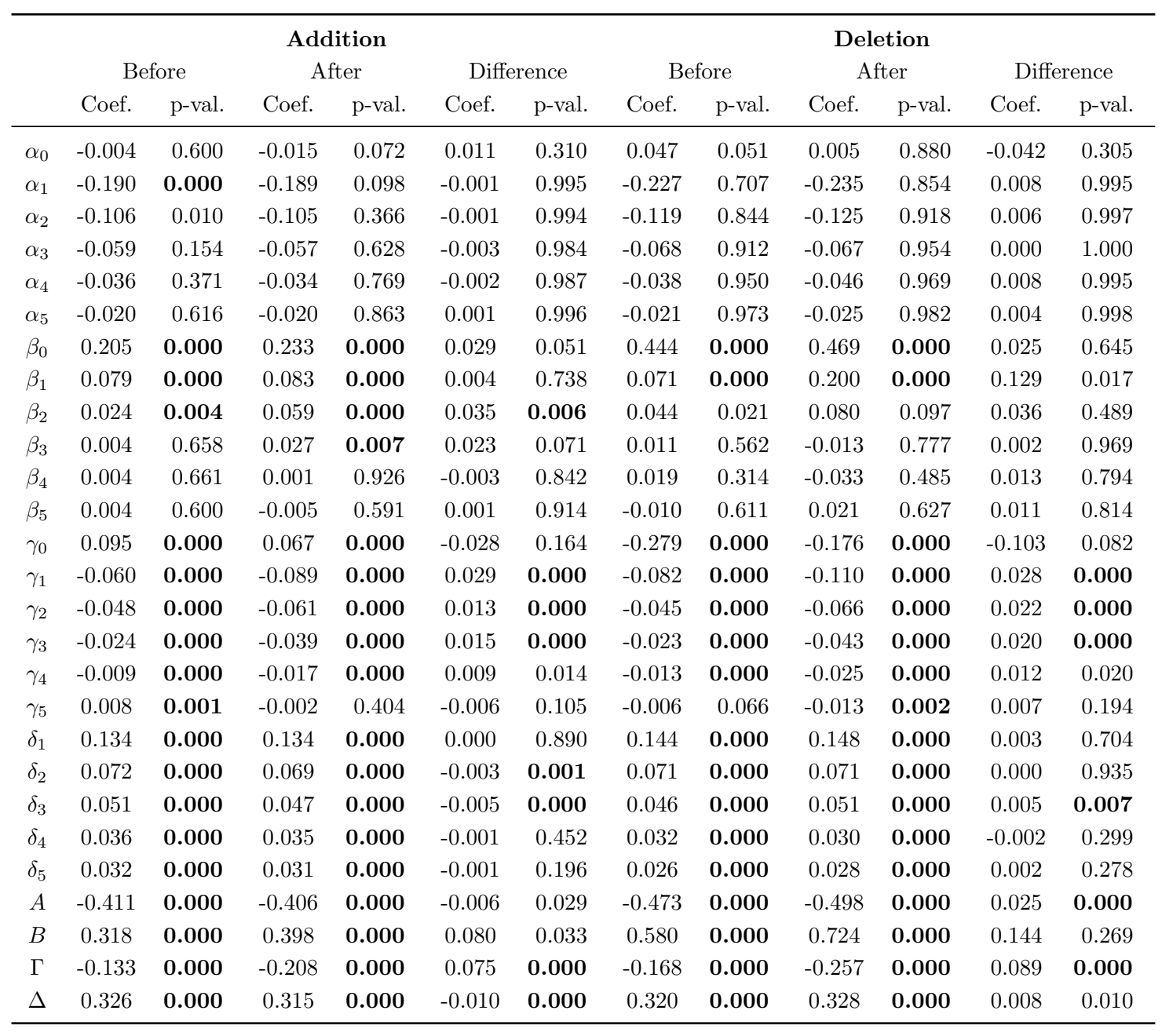




\section{Table C.19: P-Values for Tests of Model Parameters (WVAR)}

Based on the model defined at (2.1) using 60 days of trade data by WVAR. The tests for individual parameters and sums are computed as described in Section 2.3.3. The cell entries show the estimated parameters and probabilities (p-val.), respectively. Differences are calculated by absolute value of model parameters between after and before both events. Bold format denotes significance at the $1 \%$ level.

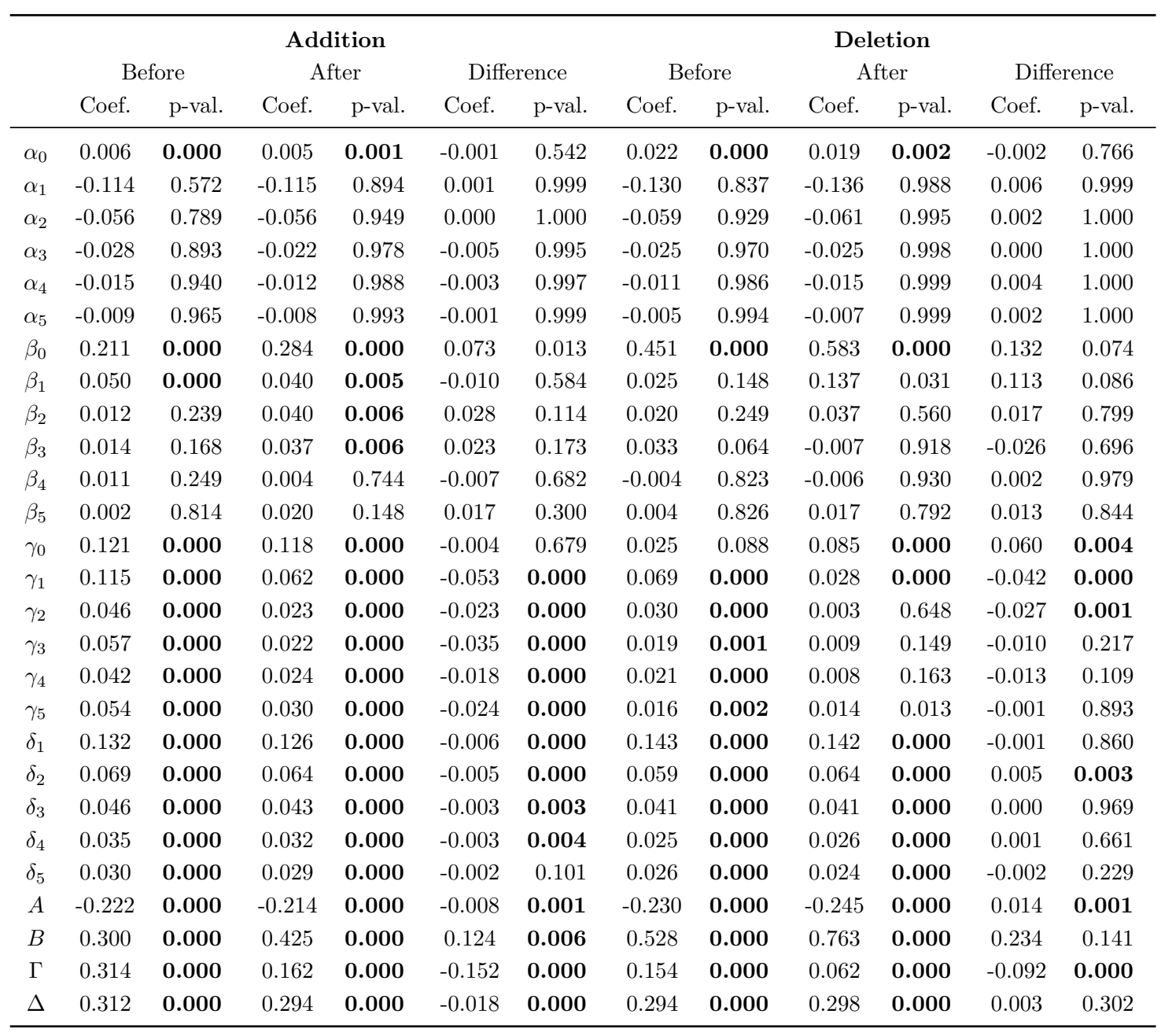


Appendix D

The Example of Data Format 


\section{Table D.1: The Example of Data Format}

The table reports the example of data format cleaned from Trade Record and Bid and Ask Record datasets. Columns are as follows: 1. Stock ID; 2. Effective date; 3. Estimated date; 4. Traded time (HH:MM:SS); 5. Traded price; 6. Traded volume; 7. Prevailing mid-quotes; 8. Subsequent mid-quotes.

\begin{tabular}{cccccccc}
\hline $\mathbf{1}$ & $\mathbf{2}$ & $\mathbf{3}$ & $\mathbf{4}$ & $\mathbf{5}$ & $\mathbf{6}$ & $\mathbf{7}$ & $\mathbf{8}$ \\
\hline 1000060 & 20020225 & 20011205 & 145657 & 0.56 & 500000 & 0.565 & 0.565 \\
1000060 & 20020225 & 20011205 & 150607 & 0.56 & 50000 & 0.565 & 0.565 \\
1000060 & 20020225 & 20011205 & 150934 & 0.56 & 252000 & 0.565 & 0.565 \\
1000060 & 20020225 & 20011205 & 151009 & 0.56 & 130000 & 0.565 & 0.565 \\
1000060 & 20020225 & 20011205 & 151708 & 0.56 & 40000 & 0.565 & 0.565 \\
1000060 & 20020225 & 20011205 & 151922 & 0.56 & 80000 & 0.565 & 0.565 \\
1000060 & 20020225 & 20011205 & 152417 & 0.57 & 100000 & 0.565 & 0.565 \\
1000060 & 20020225 & 20011205 & 152713 & 0.56 & 100000 & 0.565 & 0.565 \\
1000060 & 20020225 & 20011205 & 152822 & 0.57 & 20000 & 0.565 & 0.565 \\
1000060 & 20020225 & 20011205 & 153248 & 0.56 & 900000 & 0.565 & 0.565 \\
1000060 & 20020225 & 20011205 & 153330 & 0.56 & 50000 & 0.565 & 0.555 \\
1000060 & 20020225 & 20011205 & 153331 & 0.56 & 32000 & 0.565 & 0.555 \\
1000060 & 20020225 & 20011205 & 153332 & 0.56 & 50000 & 0.565 & 0.555 \\
1000060 & 20020225 & 20011205 & 153851 & 0.56 & 100000 & 0.555 & 0.555 \\
1000060 & 20020225 & 20011205 & 153931 & 0.56 & 160000 & 0.555 & 0.555 \\
1000060 & 20020225 & 20011205 & 154033 & 0.56 & 40000 & 0.555 & 0.555 \\
1000060 & 20020225 & 20011205 & 154046 & 0.56 & 30000 & 0.555 & 0.555 \\
1000060 & 20020225 & 20011205 & 154324 & 0.56 & 40000 & 0.555 & 0.555 \\
\hline
\end{tabular}




\section{Chapter 3}

\section{Short Sales and Price Discovery using GARCH and BEKK Models}

\subsection{Introduction}

Volatility as a measure of risk varies and has a tendency to cluster over times and this phenomenon corresponds to the fluctuations in volatility. Due to the dependence of volatility upon past realization of the asset price and the related volatility process, large swings tend to followed by large swings while small changes tend to be not far behind small changes Mandelbrot (1963). Modelling dynamic volatility has attracted much attention of academics ever since the introduction of the Autoregressive Conditional Heteroscedasticity [ARCH henceforth] model by Engle (1982). Since then numerous variants and extensions of ARCH models have been proposed. Among these, the GARCH model developed by Bollerslev (1986) is the most widely used GARCH form to describe the phenomenon of volatility clustering and capture the characteristic of heteroscedasticity in time series analysis.

While volatility modelling has been the main centre of attention, understanding the dynamic co-movements of volatility is also of great practical importance since this 
will identify volatility and correlation transmission and spillover effects from one to another. This issue can be solved by extending the considerations to multivariate GARCH [MGARCH henceforth] models. However, the curse of dimensionality becomes an issue for a MGARCH model as the dimension of volatility matrix increases rapidly when the number of variable increases. On the one hand, the specification of a MGARCH model should be flexible enough to be able to represent the dynamics of the conditional variances and covariances. On the other hand, as the number of parameters in a MGARCH model often increases rapidly with the dimension of the model, the specification should be parsimonious enough to allow for relatively easy estimation of the model and also allow for easy interpretation of the model parameters. However, parsimony may reduce the number of parameters, in which situation the relevant dynamics in the covariance matrix cannot be captured. Therefore, it is important to achieve a balance between parsimony and flexibility when designing the MGARCH model specifications. Another feature a MGARCH model must satisfy is that the covariance matrix should be positive definite. To overcome these difficulties, a BEKK model as proposed by Engle \& Kroner (1995) which is the one of the most popular MGARCH models of conditional covariances and correlations is used to investigate the volatility spillover among financial markets. The BEKK model is considered as a truncated and low-dimensional application and it ensures the positive semi-definiteness of covariance matrix.

In Chapter 2, the high frequency based quote returns and signed volumes in the VAR model of Hasbrouck (1991) invariably exhibit heterogeneity of variance and thus they could affect the model parameters which measure the price efficiency. To further investigate the impact of short sales on the speed of price adjustment under dynamic volatility and co-volatility, GARCH-based analysis is conducted in this chapter on the basis of formal econometric diagnostic tests on high frequency data. This chapter makes the following contributions to the literature. To our best knowledge, this is the first study which investigates the relationship between short sales and speed of price adjustment based on the VAR model with univariate GARCH and BEKK extensions. The univariate GARCH models help capture the volatility dynamics of individual quote returns or signed volumes. The BEKK model takes account of possible volatility spillover between quote returns 
and signed volumes. This chapter also carries out different diagnostic checking on the ARCH effect including univariate tests applied independently to each series and multivariate tests applied to the vector series as a whole. By comparing the GARCH and BEKK models with the least squares based models in Chapter 2, this chapter further concludes the preferred models to capture the effect of short sales on price efficiency are the WVAR and BEKK models.

The results are summarised as follows. First, under GARCH and BEKK frameworks, there are more stocks experiencing significant changes in parameters, including increases and decreases in the magnitude, than those under the models in Chapter 2. It suggests that more stocks' price efficiency is affected by short sales when heterogeneity of variance is taken into account for both addition and deletion events. Autocorrelations in quote revision and trades are weakened by short sales during the addition events. Quote autocorrelations become stronger during the deletion events. These findings suggest that short sales improve the price process that quote revision and trades follow a random walk with smaller absolute value of autocorrelations which are consistent with those from Boehmer \& Wu (2013). Thus, it is concluded that speed of price adjustment becomes faster by short sales when the models are estimated under heteroscedasticity. Secondly, trade autocorrelations are the most affected parameters which decrease significantly during the addition events and this is robust under all models including OLS, WLS, VAR, WVAR, GARCH and BEKK. For the deletion event, it is observed that quote autocorrelations are the most affected parameters with significant increases under the majority of models while no evidence is shown for significant changes in trade continuity. Furthermore, it is noted that WVAR and BEKK models that estimate simultaneously with consideration of heterogeneous variances are more powerful to capture the effect of short sales than other models especially for the addition events. Thirdly, a study of parameter dynamics suggests that parameters under GARCH and BEKK models remain consistent as far as sign is concerned; that is, an estimated model parameter which is significantly negative (positive) before the addition/deletion event will remain negative (positive) after the event. It is also found that, under the GARCH model, there are more stocks with consistency in the sign of parameters especially for those measuring the speed of price adjustment 
compared with OLS model.

The remainder of this chapter is organised as follows. Section 3.2 presents GARCH and MGARCH models with model diagnostics. Section 3.3 provides data description. Section 3.4 discusses the empirical results. Section 3.5 concludes the chapter.

\subsection{The Models and Diagnostic Checking}

This section summarises the univariate GARCH and BEKK models that are used in this chapter in conjunction with the VAR model of Hasbrouck (1991). There are three parts in the section. Section 3.2.1 describes the univariate GARCH model. Section 3.2.2 describes the BEKK model. Section 3.2.3 summarises the diagnostic tests that are employed.

\subsubsection{The Univariate GARCH Model}

A limitation of the ARCH model of Engle (1982) is that it often requires many lags. Bollerslev (1986) proposed the GARCH model, in which the current conditional variance is represented in terms of past squared residuals and past conditional variances. It is thus in effect an ARMA model in which the past conditional variances are the auto-regressive terms and the past squared residuals the moving averages. In common with standard ARMA models this often results in a more parsimonious parametrisation. In general a $\operatorname{GARCH}(p, q)$ model has the following form

$$
\sigma_{t}^{2}=\varphi_{0}+\varphi_{1} \varepsilon_{t-1}^{2}+\ldots+\varphi_{p} \varepsilon_{t-p}^{2}+\phi_{1} \sigma_{t-1}^{2}+\ldots+\phi_{q} \sigma_{t-q}^{2}
$$

where $\varphi_{i}>0, \phi_{j}>0, i=1, \ldots, p$, and $j=1, \ldots, q$, respectively and 


$$
1-\sum_{i=1}^{p} \varphi_{i}-\sum_{j=1}^{q} \phi_{j}>0 .
$$

The most frequently used model in finance is the $\operatorname{GARCH}(1,1)$ model, which is adopted in this chapter. In this model, the conditional variance matrix is calculated from a long-run average variance rate $\omega>0$ and from the lag terms $\varepsilon_{t-1}$ and $\sigma_{t-1}$. The univariate GARCH $(1,1)$ model can be written as

$$
\sigma_{t}^{2}=\omega+\varphi \varepsilon_{t-1}^{2}+\phi \sigma_{t-1}^{2}
$$

where $\omega>0, \varphi \geq 0, \phi \geq 0$ and $1-\varphi-\phi>0$. Applying the $\operatorname{GARCH}(1,1)$ model to the VAR model of Hasbrouck (1991), the univariate form of the model is defined as

$$
\begin{gathered}
R_{t}=\alpha_{0}+\sum_{i=1}^{5} \alpha_{i} R_{t-i}+\sum_{i=0}^{5} \beta_{i} X_{t-i}+\varepsilon_{R, t}, \\
\sigma_{R, t}^{2}=\omega_{R}+\varphi_{R} \varepsilon_{R, t-1}^{2}+\phi_{R} \sigma_{R, t-1}^{2}, \\
X_{t}=\gamma_{0}+\sum_{i=1}^{5} \gamma_{i} R_{t-i}+\sum_{i=1}^{5} \delta_{i} X_{t-i}+\varepsilon_{X, t} \\
\sigma_{X, t}^{2}=\omega_{X}+\varphi_{X} \varepsilon_{X, t-1}^{2}+\phi_{X} \sigma_{X, t-1}^{2} .
\end{gathered}
$$

where $\omega_{R}>0, \omega_{X}>0, \varphi_{R} \geq 0, \varphi_{X} \geq 0, \phi_{R} \geq 0$ and $\phi_{X} \geq 0$.

The rest of the variables in the model (3.1) above keep the same definition as Chapter 2. The variable denoted as $R$ is the difference in the natural logarithm of the mid-quotes for two successive transactions,

$$
R_{t}=\ln M_{t}-\ln M_{t-1}, M_{t}=\left(Q_{t}^{b}+Q_{t}^{a}\right) / 2,
$$


where $Q_{t}^{b, a}$ are the bid and ask quotes at time $t$. The variable denoted $X$ is the signed volume of the trade and the trade sign is defined by the classification of Lee \& Ready (1991). The quote return variable denoted $R$ is rescaled by multiplying $10^{3}$ and the trade variable denoted $X$ is rescaled by dividing by $10^{4}$. The coefficients $\alpha_{i}$ and $\delta_{i}$ are autocorrelations in quote revision and signed volume respectively. The coefficients $\beta_{i}$ indicate the impact on quote revision subsequent to each trade. The coefficients $\gamma_{i}$ capture Granger causality of lagged quote revision on trades.

Moreover, the chapter includes models from the family of first-order GARCH processes in the model selection exercise in Section 3.4.2. Based on the information criteria selection, they are compared with each other to examine their performance in capturing the time-varying volatility. These models are the GARCH-in-Mean model of Engle et al. (1987) [GARCH-M henceforth], the Integrated GARCH of Engle \& Bollerslev (1986) [IGARCH henceforth], the Exponential GARCH of Nelson (1991) [EGARCH henceforth] and the Threshold GARCH of Zakoian (1994) [TGARCH henceforth]. All models are estimated based on the same sample stocks for both addition and deletion events and each criteria information is the average across all the stock/events.

\subsubsection{The BEKK Model}

The rationale to extend the univariate $\mathrm{GARCH}$ model to a multivariate framework in financial applications is that it is considered important to be able to predict dependence in the co-movements of volatility and covariance. To ensure positive definiteness of the conditional covariance matrix, Engle \& Kroner (1995) introduced a parameterisation of the conditional variance matrix which has become known as the BEKK model. This has advantages when compared to other MGARCH specifications such as the VEC-GARCH model of Bollerslev et al. (1988). It achieves the positive definiteness of the conditional covariance matrix by formulating the 
model in such a way that this property is implied by the model structure. Unlike the dynamic conditional correlation model of Engle (2002), which estimates the time-varying correlations directly, the BEKK specification allows for time-varying correlations and also for interactions between the variances in a lead-lag framework. As an MGARCH model, the BEKK model is selected in this chapter based on a number of important considerations. The BEKK model overcomes difficulties commonly associated with the VEC-GARCH model. The VEC-GARCH model has two main problems. First, the number of parameters to be estimated under the VEC-GARCH model is large. According to Bauwens et al. (2006), the number of parameters is $N(N+1)(N(N+1)+1) / 2$ (for $N=2$ gives 24 ) where $N$ denotes the number of variables in the model. For the BEKK model, the number of parameters to be estimated is reduced to $N(5 N+1) / 2$ (for $N=2$ gives 11). The BEKK model thus reduces computational demands and improves the efficiency of parameter estimation. Secondly, the BEKK model guarantees that the conditional covariance matrix $H_{t}$ is positive definite. Furthermore, the BEKK model is superior theoretically to its diagonal model counterparts where each element of the matrix depends only on its own lagged values of shocks and volatility. The conditional covariance matrix in the BEKK model is estimated using a quasi maximum likelihood approach (Bollerslev \& Wooldridge, 1992).

The most general specification for the BEKK model is as follows

$$
H_{t}=C C^{\prime}+\sum_{j=1}^{Q} \sum_{k=1}^{K} A_{k j}^{\prime} u_{t-j} u_{t-j}^{\prime} A_{k j}+\sum_{j=1}^{P} \sum_{k=1}^{K} B_{k j}^{\prime} H_{t-j} B_{k j}
$$

where $A_{k j}, B_{k j}$ and $C$ are $N \times N$ parameter matrices, and $C$ is a lower triangular matrix and the notation ' denotes transpose. This model specification ensures that the conditional covariance matrix $H_{t}$ is at least positive semi-definite. It is generally assumed that $K=1$ to ensure identifiability. It is further assumed in financial applications that $P=Q=1$. In this case the first-order BEKK model is 


$$
H_{t}=C C^{\prime}+A^{\prime} u_{t-1} u_{t-1}^{\prime} A+B^{\prime} H_{t-1} B
$$

In matrix form, it may also be written as

$\left[\begin{array}{ll}h_{11, t} & h_{12, t} \\ h_{21, t} & h_{22, t}\end{array}\right]=C C^{\prime}+A^{\prime}\left[\begin{array}{cc}u_{1, t-1}^{2} & u_{1, t-1} u_{2, t-1} \\ u_{2, t-1} \varepsilon_{1, t-1} & u_{2, t-1}^{2}\end{array}\right] A+B^{\prime}\left[\begin{array}{cc}h_{11, t-1} & h_{12, t-1} \\ h_{21, t-1} & h_{22, t-1}\end{array}\right] B$,

with

$$
C=\left[\begin{array}{cc}
c_{11} & 0 \\
c_{21} & c_{22}
\end{array}\right], A=\left[\begin{array}{ll}
a_{11} & a_{12} \\
a_{21} & a_{22}
\end{array}\right], B=\left[\begin{array}{ll}
b_{11} & b_{12} \\
b_{21} & b_{22}
\end{array}\right]
$$

The matrix $C$ is lower triangular, $A$ and $B$ are $\mathrm{ARCH}$ and $\mathrm{GARCH}$ parameter matrices respectively. In the BEKK specification, each conditional variance and covariance in $H_{t}$ is modelled as a function of lagged conditional variances and covariances, lagged squared innovations and the cross-product of the innovations. Volatility is transmitted between quote returns and signed volume through two channels represented by the off-diagonal parameters in the ARCH and GARCH matrices: a symmetric shock $u_{i i, t-1}$ and the conditional variance $H_{i i, t-1}$.

The BEKK model applied in this chapter is based on the reduced form of the VAR model with the assumption of conditional variance and covariance. The bivariate VAR model with a first-order BEKK representation in matrix form shows is as follows

$$
\begin{gathered}
Y_{t}=\Psi_{0}+\sum_{i=1}^{5} \Psi_{i} Y_{t-i}+u_{t}, \\
H_{t}=C C^{\prime}+A^{\prime} u_{t-1} u_{t-1}^{\prime} A+B^{\prime} H_{t-1} B .
\end{gathered}
$$


where

$$
Y_{t}=\left[\begin{array}{c}
R_{t} \\
X_{t}
\end{array}\right], \Psi_{j}=\Lambda^{-1} \Phi_{j}, u_{t}=\Lambda^{-1} \varepsilon_{t}
$$

with

$$
\Lambda^{-1}=\left[\begin{array}{cc}
1 & -\beta_{0} \\
0 & 1
\end{array}\right]^{-1}, \Phi_{0}=\left[\begin{array}{l}
\alpha_{0} \\
\gamma_{0}
\end{array}\right], \Phi_{i}=\left[\begin{array}{cc}
\alpha_{i} & \beta_{i} \\
\gamma_{i} & \delta_{i}
\end{array}\right], \varepsilon_{t}=\left[\begin{array}{l}
\varepsilon_{R, t} \\
\varepsilon_{X, t}
\end{array}\right]
$$

The rest of the variables in the BEKK model defined at (3.2) above keep the same definition as in Chapter 2. The conditional covariance matrix in the BEKK model is estimated by quasi maximum likelihood method.

In summary, this chapter applies univariate GARCH models and a bivariate BEKK model to examine the effect of short sales on the speed of price adjustment with heteroscedasticity.

\subsubsection{Diagnostic Checking}

A time series exhibiting conditional heteroscedasticity is said to exhibit autoregressive conditional heteroscedastic $(\mathrm{ARCH})$ effects. Since estimating univariate GARCH and MGARCH models is time-consuming, both in terms of computations and programming, it is desirable to check whether the data present evidence of ARCH effects. There are two kinds of specification tests; namely univariate tests applied independently on each series and multivariate tests applied to the vector series as a whole. These diagnostics are summarised in this section. 


\section{Normality Test}

The normality test is used to detect misspecification of the ARCH models. Based on skewness and kurtosis, Bera \& Jarque (1982) proposed the test statistic

$$
J B_{T}=\left[\frac{T}{6} S^{2}+\frac{T}{24} K^{2}\right]
$$

where $T$ is the number of observations and

$$
S=\frac{\sqrt{T} \sum_{t=1}^{T} \hat{e}_{t}^{3}}{\left(\sum_{t=1}^{T} \hat{e}_{t}^{2}\right)^{\frac{3}{2}}}, K=\frac{T \sum_{t=1}^{T} \hat{e}_{t}^{4}}{\left(\sum_{t=1}^{T} \hat{e}_{t}^{2}\right)^{2}} .
$$

The Jarque-Bera test is distributed as $\chi_{(2)}^{2}$. When a GARCH model is estimated, the Jarque-Bera test uses the standardised estimated residuals, $\hat{e}_{t}=\hat{\varepsilon}_{t} / \hat{\sigma}_{t}$. Skewness $(S)$ and kurtosis $(K)$ may be tested separately using the two components of $J B_{T}$, each of which is distributed as Chi-squared with one degree of freedom.

\section{Portmanteau $Q$ Test}

For nonlinear time series models, the portmanteau $Q$ test statistic based on squared residuals is used to test for independence for the series (McLeod \& Li, 1983)

$$
Q(q)=T(T+2) \sum_{i=1}^{q} \frac{r\left(i ; \hat{\varepsilon}_{t}^{2}\right)}{(T-i)}
$$

where $T$ is the number of observations and 


$$
r\left(i ; \hat{\varepsilon}_{t}^{2}\right)=\frac{\sum_{t=i+1}^{T}\left(\hat{\varepsilon}_{t}^{2}-\hat{\sigma}^{2}\right)\left(\hat{\varepsilon}_{t-i}^{2}-\hat{\sigma}^{2}\right)}{\sum_{t=1}^{T}\left(\hat{\varepsilon}_{t}^{2}-\hat{\sigma}^{2}\right)^{2}}, \hat{\sigma}^{2}=\frac{1}{T} \sum_{t=1}^{T} \hat{\varepsilon}_{t}^{2} .
$$

This $Q$ statistic is used to detect the nonlinear effects present in the residuals. The GARCH $(p, q)$ process can be considered as an ARMA $(\max (p, q), p)$ process. The $Q$ statistic calculated from the squared residuals can be used to identify the order of the GARCH process.

\section{Lagrange Multiplier Test for ARCH Disturbances}

Engle (1982) proposed a Lagrange multiplier test for ARCH disturbances. The test statistic is asymptotically equivalent to the test used by Breusch \& Pagan (1979). Engles Lagrange multiplier test for the $q^{\text {th }}$ order ARCH process is written as

$$
L M(q)=\frac{T W^{\prime} Z\left(Z^{\prime} Z\right)^{-1} Z^{\prime} W}{W^{\prime} W}
$$

where $T$ is the number of observations, $W$ is a vector of standardised squared residuals

$$
W=\left(\frac{\hat{\varepsilon}_{1}^{2}}{\hat{\sigma}^{2}}, \ldots, \frac{\hat{\varepsilon}_{T}^{2}}{\hat{\sigma}^{2}}\right)^{\prime}
$$

and

$$
Z=\left[\begin{array}{cccc}
1 & \hat{\varepsilon}_{0}^{2} & \ldots & \hat{\varepsilon}_{-q+1}^{2} \\
\vdots & \vdots & \vdots & \vdots \\
\vdots & \vdots & \vdots & \vdots \\
1 & \hat{\varepsilon}_{T-1}^{2} & \cdots & \hat{\varepsilon}_{T-q}^{2}
\end{array}\right]
$$


The presample values $\left(\varepsilon_{0}^{2}, \ldots, \varepsilon_{-q+1}^{2}\right)$ have been set to 0 . The $L M(q)$ and $Q$ statistics are computed from the OLS residuals assuming that disturbances are white noise. The $L M(q)$ and $Q$ statistics have an approximate $\chi_{(q)}^{2}$ distribution under the white-noise null hypothesis.

\section{Tests for Multivariate GARCH Models}

Compared to the diagnostic tests devoted to univariate models, there are few tests on ARCH effects specific to multivariate models. The most widely used to detect whether the model residuals remain correlated are probably the Box-Pierce/LjungBox portmanteau $Q$ tests. A multivariate version of the Ljung-Box test statistic following Hosking (1980) is given by

$$
L B(L)=T(T+2) \sum_{j=1}^{L}(T-j)^{-1} \text { trace }\left\{\hat{P}_{0 j} \hat{P}_{00}^{-1} \hat{P}_{0 j}^{\prime} \hat{P}_{00}^{-1}\right\}
$$

where $\hat{P}_{0 j}=T^{-1} \sum_{t=j+1}^{T} \hat{u}_{t} \hat{u}_{t-j}^{\prime}, \hat{u}_{t}$ is the estimated vector of residuals at time $t, L$ is the order of autocorrelation, and $T$ is the number of observations.

Under the null hypothesis that the residuals are uncorrelated, the Ljung-Box test statistic has approximately a Chi-squared distribution with $n^{2}(L-p)$ degrees of freedom where $n$ is the number of equations and $p$ is the lag length in the model. It is important to note that the test can be implemented only when the order of autocorrelation is higher than the lag length in the model, i.e. $L>p$. 


\subsection{Data Description}

This chapter uses the same intraday data of Chapter 2, which covers a period of 10 years from May 2001 to May 2011. An addition (deletion) event is defined when an individual stock is added to (removed from) the D-list for short sales from the effective date. Re-entry (re-quit) of an individual stock is considered as a separate addition (deletion) event. The 10-year period intraday data excludes unit trusts, exchange traded funds, mutual funds, investment companies and stocks traded on the Growth Enterprise Market (GEM). First time IPO firms in the addition events are excluded as there are inadequate observations in the pre-event period. Stocks with options and futures written are excluded as option and future trading can be considered as an alternative to short selling but at a lower cost (Diamond \& Verrecchia, 1987).

The estimation in this chapter contains two models under heteroscedasticity including $\operatorname{GARCH}(1,1)$ and $\operatorname{BEKK}(1,1)$ models, using all transactions in the 60-day period before and after the event date in the 10-year period. It should be noted that there is no requirement to specify weighted GARCH or BEKK models. This is because the variance dynamics equation implicitly allows for the effect of the time gap between trades and it can therefore be argued that weighted GARCH or BEKK models would be over specified as weighted methodology is considered as an alternative solution to heteroscedasticity. Stocks for which estimation errors occurred using GARCH and BEKK models are excluded, which results in the reduction of the sample of this chapter to 1,082 addition events and 690 deletion events $^{12}$.

\footnotetext{
${ }^{12}$ For a small number of stock/events, the software fails to achieve convergence for BEKK or GARCH estimation.
} 


\subsection{Results}

This section presents the results using the 60-day trade data based on 1,082 addition events and 690 deletion events with two model estimations including GARCH and BEKK. The SS test used in this chapter is defined the same as Chapter 2 and the significance level is 1\%. Section 3.4.1 reports the diagnostic tests for ARCH effects for the univariate model (OLS) and the multivariate model (VAR) respectively using the diagnostic checking methods introduced in Section 2.3 above. Section 3.4.2 reports the selection criteria for different GARCH models to approve the model selections. Section 3.4.3 reports the model validation tests using the same procedure in Chapter 2. Section 3.4.4 presents the results about changes in model parameters as a result of addition/deletion event. Sections 3.4.5 demonstrates the results on dynamics and p-values of model parameters for both events. Sections 3.4.6 reports the $Z$ scores for tests on difference in model parameters for both events.

\subsubsection{Diagnostic Tests for ARCH Effects}

For addition events, Table 3.1 reports the diagnostic tests results based on OLS, showing the percentage of event stocks at the corresponding significance levels which are $0.1 \%, 1 \%$ and $5 \%$. Any results with a significance level greater than $5 \%$ are classified as neutral. The test is used to test for normality of residuals. The Portmanteau $Q$ test and the Lagrange Multiplier Test ( $L M$ test) are used to test the significance of ARCH effects which is concerned with a relationship within the heteroscedasticity. Numbers in the brackets denotes the number of lags of the residual series. Four vertical sections are for $R$ and $X$ equations in the model before and after the event. Bold format in the table denotes the greatest percentage value.

[Insert Table 3.1 about here]

Table 3.1 shows that the residuals of nearly $99 \%$ of the sample stocks do not follow a normal distribution. At least $67 \%$ and $43 \%$ of the sample stocks experience ARCH 
effects at a significance level of $1 \%$ as shown by the Portmanteau $Q$ test and the $L M$ test for $R$ and $X$ equations, respectively. Furthermore, there are more stocks with corresponding significant ARCH effects in the equation before and after the addition events compared with the $X$ equation. The results in Table 3.2 report the similar results for deletion events. Although the percentage of event stocks with significant $\mathrm{ARCH}$ effects reduces in the $X$ equation compared to the addition events, there are still more than $32 \%$ of the sample stocks in the $X$ equation and $68 \%$ in the $R$ equation which present evidence of $\mathrm{ARCH}$ effects at the significance level of $1 \%$.

[Insert Table 3.2 about here]

To perform diagnostic checking for MGARCH models, we use the residuals from the VAR model of Chapter 2 and apply the multivariate Portmanteau $Q$ test to test whether correlation remains. The multivariate Portmanteau $Q$ test is valid only when lags are larger than the model's lag order, which is 5 in this thesis. In addition, the univariate Normality Test ( $J B$ test) is also used to test for normality of residuals in each equation. The $F$ tests for $A R$ disturbance ( $A R$ test) are used to test whether the residuals of the univariate $A R(1), A R(1,2), A R(1,2,3)$ and $A R(1,2,3,4)$ models are uncorrelated. The $F$ test for autoregressive conditional heteroscedastic disturbances ( $A R C H$ test) is used to test whether the residuals have equal covariances. Table 3.3 reports the diagnostic tests for ARCH effects under the VAR model for addition events.

[Insert Table 3.3 about here]

The $J B$ test results in Table 3.3 show that nearly all the sample stocks' residuals in $R$ and $X$ equations do not follow a normal distribution. All residuals of the univariate $A R(1), A R(1,2), A R(1,2,3)$ and $A R(1,2,3,4)$ models are uncorrelated. The univariate $A R C H$ test shows that more stocks' residuals exhibit significantly unequal covariances in $R$ equations than that in $X$ equations. The multivariate 
Portmanteau $Q$ test indicates that there are cross correlations remaining on the model residuals for more than approximately half of the addition stocks at a significance level of 1\%. Table 3.4 reports the similar results for ARCH effects under the VAR model for deletion events. At least $71 \%$ (35\%) of the sample stocks have unequal covariances at the significance level of $1 \%$ in the $R(X)$ equation during the event according to the univariate $A R C H$ test. The percentage from the multivariate Portmanteau $Q$ test for cross correlations of residuals at significance level of $1 \%$ remains between $25 \%$ and $37 \%$ during deletion events.

[Insert Table 3.4 about here]

In a sum, the results from the diagnostic tests under univariate and multivariate frameworks suggests that in general more than half of sample stocks experience significant ARCH effect during both events under OLS and VAR models. The only exception is those stocks under VAR for deletion events with percentage of $31 \%$ for ARCH effect at the significance level of $1 \%$. Therefore, GARCH and MGARCH frameworks are applicable with OLS and VAR to capture dynamic volatility and comovements of volatility in the analysis on the effect of short sales on price efficiency.

\subsubsection{Selection Criteria for Different GARCH Models}

In addition to the diagnostic tests for $\mathrm{ARCH}$ effects, this section provides the model selection criteria for different GARCH models. Model selection covers GARCH (1, 1), IGARCH $(1,1)$, EGARCH $(1,1)$, GARCH-M $(1,1)$ and TGARCH $(1,1)$ models. The selection criteria used are Akaikes information criterion $(A I C)$, corrected Akaikes criterion $(A I C C)$, Schwartz Bayesian criterion $(S B C)$, Hannan-Quinn criterion $(H Q C)$ and $R^{2}$. Results are reported for both $R$ and $X$ equations and for addition and deletion events. Each model selection criterion is calculated for each stock for each event using the 60-day estimation window. The results shown in the tables are averages computed across all stock/events. 
[Insert Tables 3.5 and 3.6 about here]

Tables 3.5 \& 3.6 present the selection criteria for different GARCH models for addition and deletion events. Among these competing GARCH models, the model deemed to be the best model has the smallest value of the information criterion in question. As both Tables 3.5 and 3.6 shown, the average differences in the value for each selection criterion are small. Therefore, no single GARCH model appears to be systematically superior and so the standard GARCH $(1,1)$ model is used along with the standard $\operatorname{BEKK}(1,1)$ model.

\subsubsection{Model Validation}

A consistency check of the parameters is conducted using the same procedure as in Chapter 2. The $Z$ statistic defined in Chapter 2 has a standard normal distribution under the null hypothesis $H_{0}: \Omega=0$ and rejection of the null hypothesis could indicate inconsistency of the model. Table 3.7 reports the percentage of occasions for which the model consistency test based on equation (2.2) yields results at the specified level of significance under $\operatorname{GARCH}(1,1)$ and $\operatorname{BEKK}(1,1)$ models as well as four models in Chapter 2 including OLS, WLS, VAR and WVAR for both addition and deletion events. Compared with the results under models from Chapter 2 , the table shows that the model is not validated at the $0.5 \%$ level of significance for between $2 \%$ and $5 \%$ of all stock/events. The overall $Z$ scores in the last column of the table are all less than one in magnitude, indicating that overall the models with GARCH and BEKK extensions may be considered to be validated. Tables for the detailed number of model validation under GARCH and BEKK are available in Appendix E.

[Insert Table 3.7 about here] 


\subsubsection{Changes in Model Parameters}

Changes in the estimated parameters using the GARCH and BEKK models are reported in this section. There is also a comparison with the corresponding tables from Chapter 2 at the end of the section. Table 3.8 is related to addition events using the GARCH $(1,1)$ model. The table has four vertical sections and two horizontal panels. The first panel shows results for individual parameters. The second panel shows results for the sums of the $\alpha \mathrm{s}(A), \beta_{\mathrm{s}}(B), \gamma_{\mathrm{s}}(\Gamma)$ and $\delta \mathrm{s}$ $(\Delta)$ respectively. The first vertical section show the numbers of stocks for which the corresponding parameter is significantly less than zero, neutral or significantly greater than zero before the addition event. The second vertical section shows the corresponding results after the addition event. The section headed "Chsq"shows the value of a Chi-squared statistic that is used to test the difference between the observed frequencies in the before and after period for each variable. The last vertical section shows the results for the differences in parameters during addition events and it shows the numbers of stocks for which the corresponding parameter exhibits significant decrease, neutral change or significant increase in its magnitude during addition events. Differences are calculated by absolute value of model parameters between after and before addition events. The significance level is defined at the $1 \%$. Cells in which there is a substantial number $(108,10 \%$ of the 1,082 addition events or $69,10 \%$ of the 690 deletion events) of statistically significant changes are shown in bold.

[Insert Table 3.8 about here]

Figures in bold in Table 3.8 show that there are a great number of significant changes in the first two lags of $\alpha_{i}$ (measuring the quote autocorrelations), the first lag of $\beta_{i}$ with the current $\beta_{0}$ (measuring the price impact of trades) and the first lag of $\delta_{i}$ (measuring the trade autocorrelations). For instance, 764 stocks ( $70.6 \%$ of addition stocks) experience significant changes in $\beta_{0}$ including 344 decreases and 420 increases. The individual changes result in many corresponding significant changes in the sums $(A, B$ and $\Delta$ ) except the sum $\Gamma$ which measures the Granger causality of aggregated lagged quote revision on trades. Overall, the coefficients $\alpha_{i}, \beta_{i}, \delta_{i}$ 
and their sums exhibit a substantial number of changes during the addition events using the GARCH $(1,1)$ framework. The Chi-squared scores reports that none of the sets of parameters before and after the addition events experience significant differences. This means that the signs of model parameters for the majority stocks are consistent during the event although many significant changes including both increase and decrease occurred.

The corresponding results for deletion events by GARCH $(1,1)$ are presented in Table 3.9 using 69 stocks (10\% of the 690 deletion events) as a threshold. The table shows the similar results with smaller percentage of significant changes for fewer parameters compared with the addition events.

[Insert Table 3.9 about here]

For the BEKK model, figures in bold in Table 3.10 show that a number of stocks are significantly affected during the addition events and these significant increases and decreases occurred among parameters measuring quote returns autocorrelations, price impact of trades and trade autocorrelations expect for those measuring Granger causality. In addition, more lagged parameters measuring price impact of trades and trade continuity experience significant decline rather than significant increase and it suggests that the magnitude of more parameters gets weaken by short sales under BEKK framework compared with GARCH. The insignificant Chi-squared scores approve the consistency in the sign of parameters under BEKK framework during the addition events. Table 3.11 shows the corresponding results for deletion events under the BEKK. The table provides similar results that there are fewer significant changes and fewer parameters affected during deletion events rather than addition events.

[Insert Tables 3.10 and 3.11 about here] 
Based on the results from the GARCH and BEKK models, it is concluded that there is a number of stocks with significant parameter changes when time-varying volatility and co-movements of volatility are incorporated in the underlying VAR model. For those stocks that are significantly affected, the parameters exhibiting substantial changes are those measuring quote revision autocorrelations, price impact of trades and trade autocorrelations. However, the overall effect of short sales on model parameters is not clear as significant changes contain positive and negative ones during both events.

Table 3.12 presents a comparison of the summary results for the sums denoted $A$, $B, \Gamma$ and $\Delta$ from Tables 3.8 and 3.10 with the corresponding tables under OLS and VAR in Chapter 2 for addition events. The table entries are shown as percentages as the size of the data sets for GARCH and BEKK models are not the same as those for OLS and VAR models. The table shows that the percentage changes are broadly similar for all four models for addition events. It can be noted however that the summary results under OLS are somewhat different from the other three models especially in the sums of parameters $A$ and $\Delta$ measuring the speed of price adjustment. It is also reported that GARCH and BEKK models capture more significant decreases and increases during the event in all aggregated parameters compared with OLS and VAR and this finding suggests that models with GARCH and BEKK extensions are more powerful in capturing the changes in the autocorrelations in quote returns and trades on the high frequency basis. Table 3.13 reports the similar comparison results for deletion events that there are more significant changes in parameters are observed under models with consideration of heteroscedasticity during the event.

[Insert Tables 3.12 and 3.13 about here] 


\subsubsection{Parameter Dynamics}

In this section, we carry out a study of parameter dynamics to examine the consistency in the sign of model parameters before and after addition and deletion events. Table 3.14 shows parameter dynamics using the GARCH model for both addition and deletion events. Each panel shows the results of estimates of the sums of the $\alpha \mathrm{s}, \beta \mathrm{s}, \gamma \mathrm{s}$ and $\delta \mathrm{s}$ respectively. Rows of each panel are before the event and columns are after it. "N" and "P" denotes negative and positive respectively. All other changes at a significance level of which is greater than $5 \%$ are classified as neutral. The horizontal and vertical direction of each significance category denote before and after the event, respectively. The table is divided vertically into two sections for addition and deletion events. The predominant cells are shown in bold ${ }^{13}$.

In the first row of the addition section, $92.98 \%$ of stock/events have an estimated value of parameter $A$ (quote revision autocorrelations) which is significantly less than zero at $1 \%$ level before and after the addition event. In the deletion section, the last row of the table shows that $2.61 \%$ of sample stocks have an estimated value of parameter $\Delta$ (trade autocorrelations) which is positively significant at the $5 \%$ level before the event and is positively significant at the $1 \%$ level after the event. The results show that the majority of the sums of estimated model parameters, with the exception of the sum $\Gamma$ (measures Granger causality), have consistent signs during both addition and deletion events for both events. Furthermore, for the sums of $A, B$ and $\Delta$, the table points that the percentages of the stocks with consistent signs at the significance level of $1 \%$ are higher for the addition events than those for the deletion events. It implies that more stocks exhibit consistency in the sign of these sums of parameters during the addition events rather than deletion events. The results for the BEKK model in Table 3.15 are consistent with those from the GARCH model. It is concluded that the majority of stocks have significant consistency in the signs of the sums of $A, B$ and $\Delta$ for both events and both models.

\footnotetext{
${ }^{13}$ More detailed tables which cover individual parameters are available on request.
} 
The differences in sign consistency between addition and deletion events can be linked to prospect theory in behavioural finance introduced by Kahneman \& Tversky (1979), namely that the reaction to good news (addition events) should be different from the reaction to bad news (deletion events). Kahneman \& Tversky (1979) explain particular forms of irrational behaviours of investors by stating that "The value function is normally concave for gains, commonly convex for losses, and is generally steeper for losses than for gains". A consequence of behavioural finance is that investors will react irrationally to deletion events which are perceived as bad news and which make investors nervous and lack confidence. It is conjectured that these behavioural effects are the cause of less parameter consistency for deletion events.

[Insert Tables 3.14 and 3.15 about here]

Table 3.16 reports a summary of key cells of parameter dynamics for the sums denoted $A, B, \Gamma$ and $\Delta$ under OLS, WLS, VAR, WVAR, GARCH $(1,1)$ and BEKK $(1,1)$ for addition and deletion events. The table entries are shown as percentages of stocks with consistency in the sign of model parameters before and after both events. The table shows that there are more stocks with consistency in the sign of parameters during addition events than deletion events. For each event, less stocks exhibit consistency when models are estimated on a time-weighted basis especially for the sums $A, B$ and $\Delta$. Furthermore, it is noted that the consistency in the sign of autocorrelations in quote returns $(A)$ and trades $(\Delta)$ remains stronger under GARCH model compared with OLS model.

[Insert Table 3.16 about here] 


\subsection{6 $Z$ Scores for Tests of Difference in Model Parame- ters}

The results from Section 3.4.4 reveal that more stocks are observed to experience significant changes in the model parameters under GARCH and BEKK frameworks during both events however it is not clear to determine the effect of short sales on the speed of price adjustment as these changes contain both increases and decreases in the corresponding parameters.

To investigate the overall effect of short sales on price efficiency, Table 3.17 summarises the $Z$ scores for tests of the differences in estimated model parameters using 60 days of trade data under $\operatorname{GARCH}(1,1)$ and $\operatorname{BEKK}(1,1)$ frameworks as well as four models from Chapter 2 during addition events. Differences are calculated by absolute value of model parameters between after and before both events (After minus Before). The $Z$ scores for the differences are computed using the SS test statistic at (2.10) defined in Chapter 2. There are six sub-sections corresponding to the test results for the six models. $Z$ scores shown in bold in the table are significantly different from zero at the $1 \%$ level for a two-tailed test.

[Insert Table 3.17 about here]

To compare GARCH with BEKK, it is observed that more individual and aggregated model parameters especially for $\alpha_{i}$ and $\delta_{i}$ are affected significantly under BEKK model compared with GARCH model during the addition events. For quote revision autocorrelations, it is found that more individual lagged parameters $\alpha_{i}$ experience changes at the $1 \%$ significance level under BEKK model. Only the aggregated quote autocorrelation parameter $A$ under BEKK model experiences a significant decrease during the addition events and such reduction in the magnitude of quote autocorrelations indicates a faster speed of price adjustment enhanced by short sales. For trade continuity, the majority of significant decreases in the first three lagged trade autocorrelations, $\delta_{i}$ are observed in BEKK estimations. The aggregated trade autocorrelation parameters $\Delta$ decrease at the significance level 
of $1 \%$ under both GARCH and BEKK models and this confirms that short sales fasten the speed of price adjustment by weakening autocorrelations in trades. The majority of the lagged parameters $\beta_{1}, \ldots, \beta_{5}$ do not have significant changes under BEKK and GARCH models and these results in the insignificant differences in the aggregated price impact parameter $B$ during the event. The finding shows that the effect of short sales on price impact of trades disappears when heteroscedasticity is considered. Significant increases are found in the individual and aggregated values of Granger causality under GARCH and BEKK models.

By comparing results above with those under models from Chapter 2 for the addition events, the aggregated trade continuity decreases throughout six models and it suggests that trades become less correlated after being added to the D-list with robustness under all models. The aggregated autocorrelations in quote returns decrease at the significance level of $1 \%$ under WVAR and BEKK and at the significance level of $5 \%$ under VAR. It implies that short sales improve price efficiency by weakening the autocorrelations in quote returns while the effect is only captured when the model is estimated simultaneously. The aggregated price impact of trades increase significantly under WLS and WVAR and it suggests that the price impact of trades is enhanced by short sales when time-duration is considered. The Granger causality experience significant changes at the significance level of $1 \%$ under all models during the addition events. However, it is not clear to conclude the effect of short sales on Granger causality as these changes contain both increases under unweighted models and decreases under weighted models.

Table 3.18 summarises the corresponding $Z$ scores for test of the differences in model parameters under six models for the deletion events.

[Insert Table 3.18 about here]

For results under GARCH and BEKK models, there are less model parameters with significant changes compared with the addition events. For quote revision, a decline in the value of the first lagged parameter $\alpha_{1}$ at the $1 \%$ significance level 
is observed and it is also the main attribution to the significant decline in the aggregated autocorrelations in the sum $A$ under BEKK model. It shows that quote returns are more correlated if stocks are removed from the short-selling list and it is consistent with the hypothesis that short sales constraints delay the speed of price adjustment. The individual and aggregated trade autocorrelations do not have significant changes under GARCH and BEKK and it indicates that trade continuity is not affected by short sales significantly during the deletion events. Similar results are also obtained for price impact of trades for deletion stocks. There is a significant increase in the aggregated Granger causality attributed by the increase in values of lagged parameter $\gamma_{i}$ under GARCH and BEKK models. However, combined with the findings on Granger causality parameters for the addition events, the effect of short sales on Granger causality remains unclear.

Compared with results under models from Chapter 2, the aggregated quote autocorrelations increase under the majority of models. It indicates that the quote autocorrelations are the most affected parameters and the speed of price adjustment gets slower during the deletion events. However, there is no significant evidence that trade continuity is affected when stocks lose their eligibility of short sales and the findings are robust under all models. Similarly, price impact of trades do not change significantly under these six models. Consistent with the results from the addition events, the effect of short sales on Granger causality is inconclusive.

To conclude, it is found that autocorrelations in quote returns and trades decrease significantly during the addition events while only quote autocorrelations have significant increase during the deletion events under both GARCH and BEKK models. The results suggest that the faster speed of price adjustment after the addition events is attributed to the decreased autocorrelations in both quote returns and trades however the slower speed of price adjustment during the deletion events is mainly due to the increased quote autocorrelations. However, there is no remarkable evidence to support the effect of short sales on price impact of trades and Granger causality when time-varying variance is considered. 
The overall results under six models indicate that more parameters are affected during the addition events than the deletion events. Among these changes, trade continuity is the most affected parameter weakened by short sales than quote autocorrelation during the addition events while quote autocorrelation is the most affected one to be strengthened during the deletion events. Tables for p-values for tests of individual and aggregated model parameters before and after both events under GARCH and BEKK models are available in Appendix E.

\subsection{Conclusions}

This chapter carries out an investigation on the effect of short sales on the speed of price adjustment by extending the VAR model to univariate GARCH and MGARCH models with consideration of heteroscedasticity which may be caused by high frequency trade-by-trade data in the Hong Kong stock market. In this chapter, it applies a bivariate VAR model with the extensions of $\operatorname{GARCH}(1,1)$ and BEKK $(1,1)$ models. The univariate GARCH model captures dynamic volatility while the BEKK model captures co-movements of volatility between quote returns and trades. Furthermore, the BEKK model has advantages compared with other MGARCH models as its quadratic forms ensure the positive definiteness of the conditional covariance matrices.

Based on the intraday data over 10 years, the main conclusions of this chapter are as follows. First, there are a number of stocks for which certain parameters in the model do exhibit significant changes including increases and decreases in both directions during both events under GARCH and BEKK models. To compare with OLS and VAR models assuming that the residuals have homogeneous variance, more significant changes are captured under models with consideration of heterogeneous variances on a high frequency basis. Second, the results from parameter dynamics suggests that the majority of model parameters stay consistent during both events. For example a positive parameter that is statistically significant will generally remain in the same category, even if the estimated value changes as a 
result of the addition/deletion event. It is reported that there is a stronger consistency especially for the parameters $A$ and $\Delta$ as proxies for price efficiency under GARCH model rather than OLS model.

For the overall effect of short sales on the speed of price adjustment, the results show that short sales play an important role in recovering price efficiency during both events when volatility and co-volatility are considered and the findings are consistent with those under the models from Chapter 2. Combined with the results for Chapters $2 \& 3$, it is noted that the parameters which measure trade continuity are affected most during the addition events and the trade stickiness is weakened by short sales with robustness under all models. The significant decrease in the value of quote autocorrelations under WVAR and BEKK implies that quote returns are less correlated after being added to the D-list which is consistent with those from Boehmer \& Wu (2013). For the deletion events, quote autocorrelations are the most affected parameters under the majority of model estimations and the significant increase in the value of parameters indicates that quote returns are more likely to follow the historical quote returns when stocks are not allowed for short sales. The results also show that trade continuity is not affected significantly during the deletion events.

Overall, results from six models show that short sales enhance price efficiency by reducing (increasing) autocorrelations in quote returns and trades during the addition (deletion) events. Moreover, parameters as proxies for price efficiency including autocorrelations in quote returns and trades are affected to different extent under a variety of models during different events. The combined results reveal that WVAR and BEKK models, that is models estimated simultaneously and with consideration of heterogeneous variances, have more power in ability to capture the changes in parameters measuring price efficiency than other models especially when stocks are added to the D-list for short sales. However, particularly at the aggregate level, the differences between all six models reported in Chapters $2 \& 3$ are not great and, as the chapter will show, the univariate or equation by equation approach may be used for some of the more detailed investigations. 
The next chapter plans to extend the empirical analyses on the determinants of the effect of short sales on price efficiency by investigating market returns, market capitalisation, trading volume and shorting activities. 

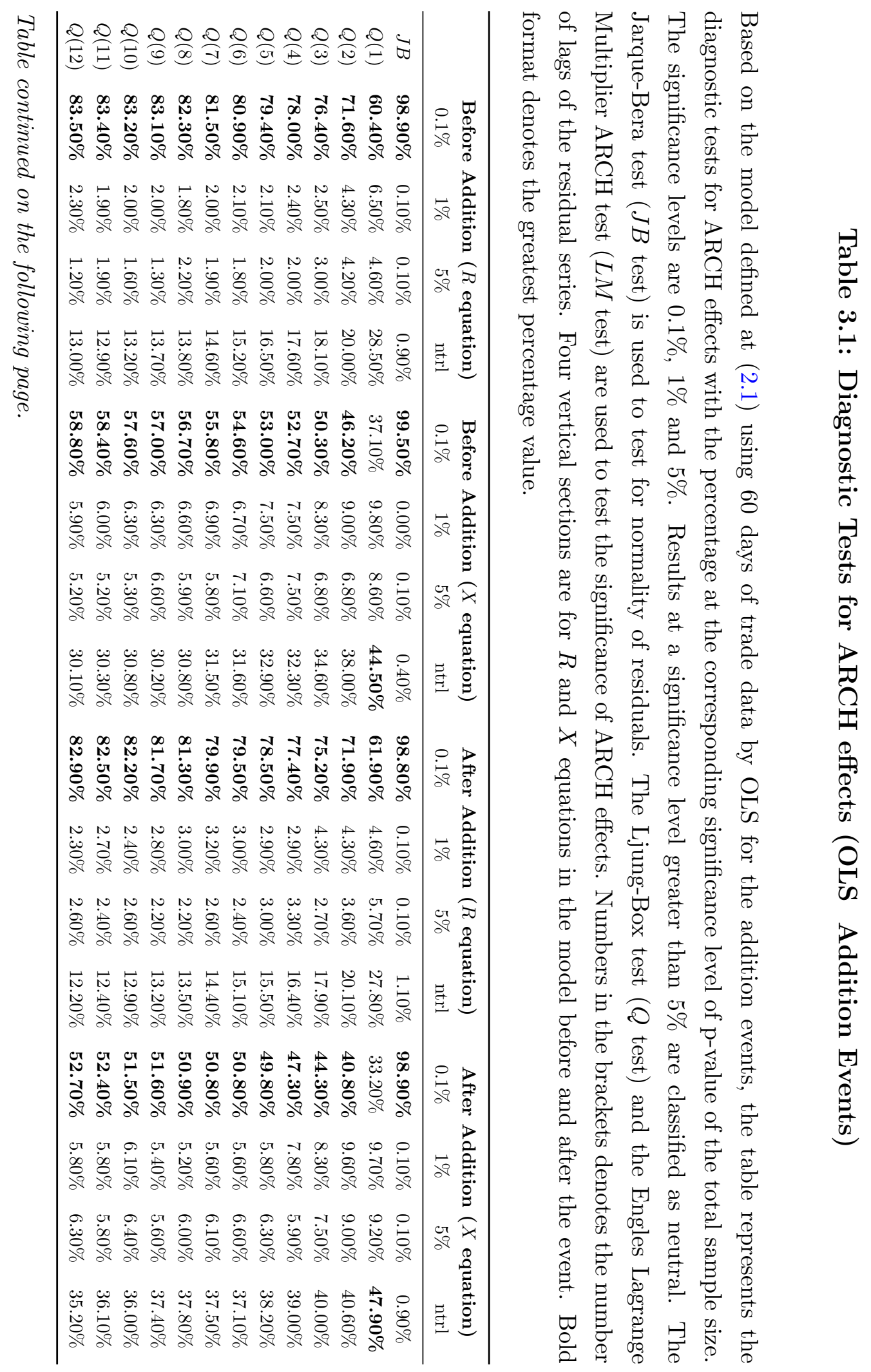


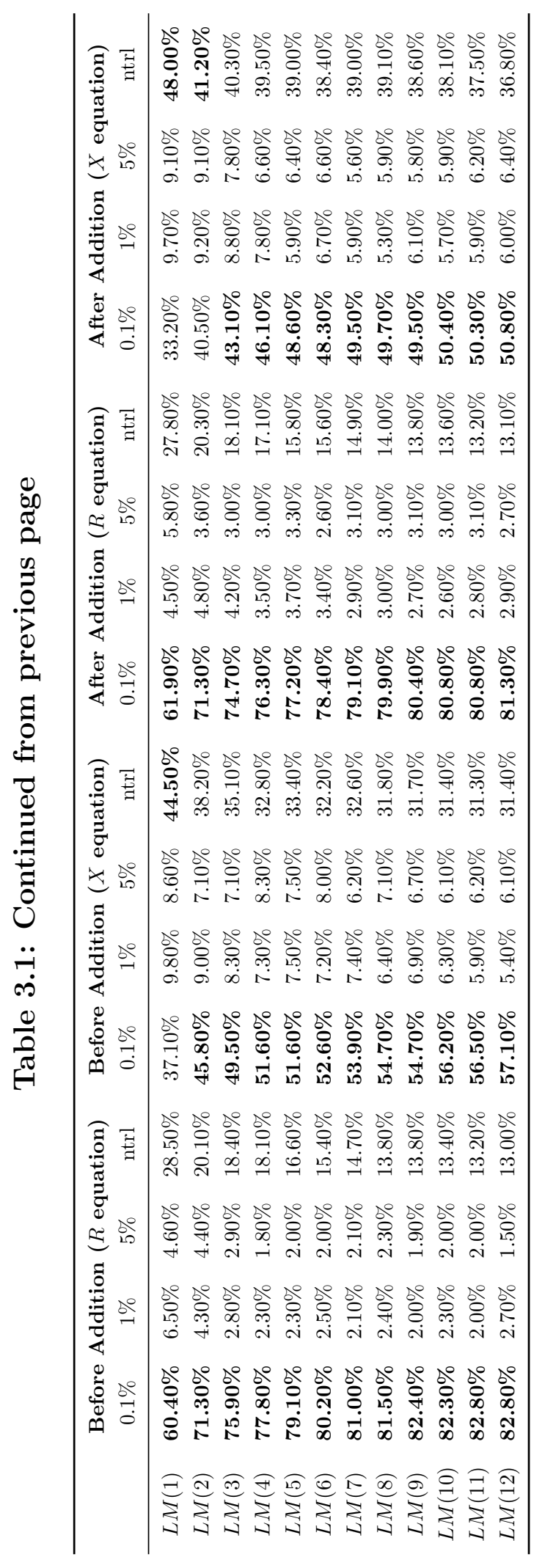



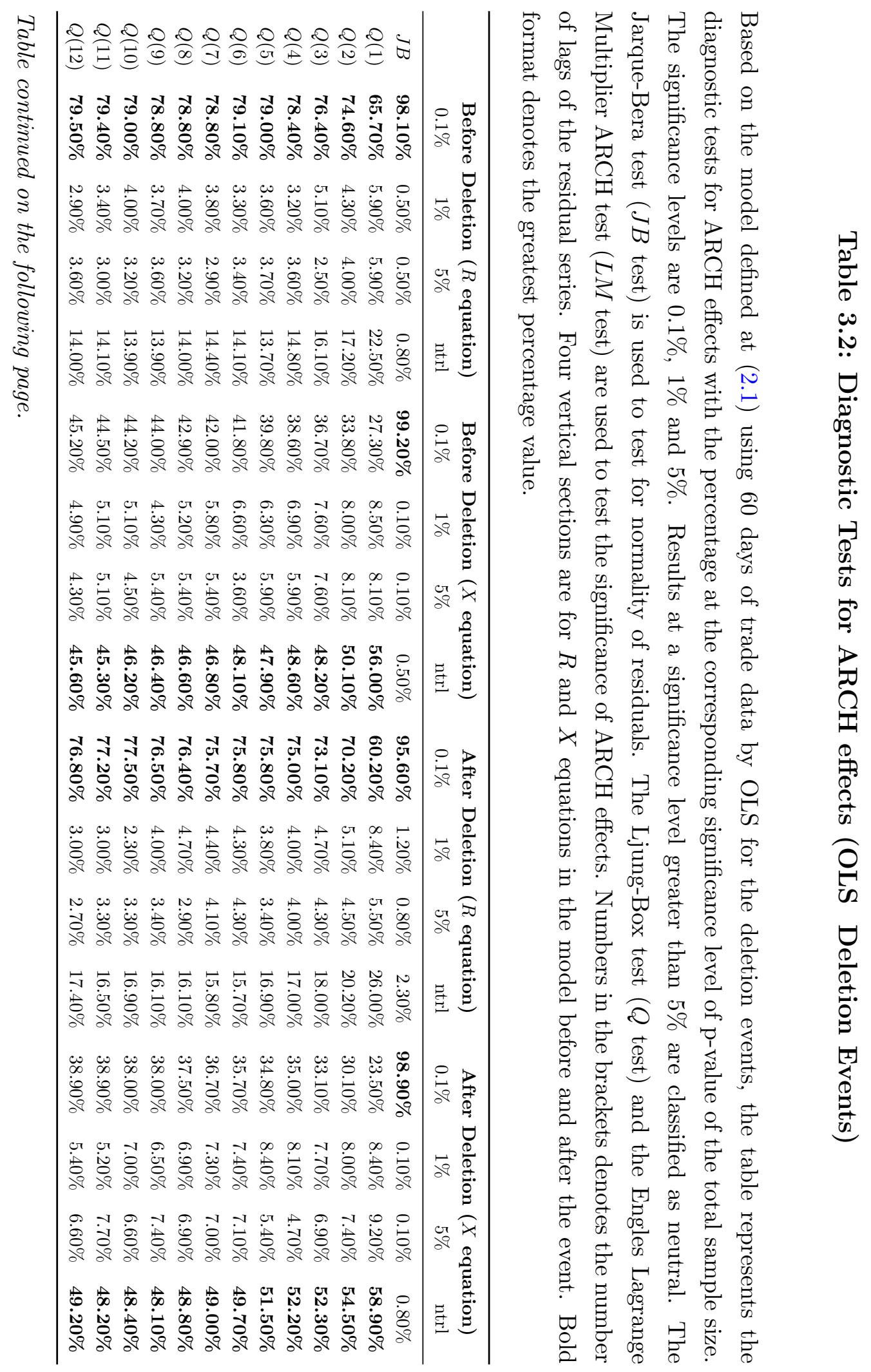


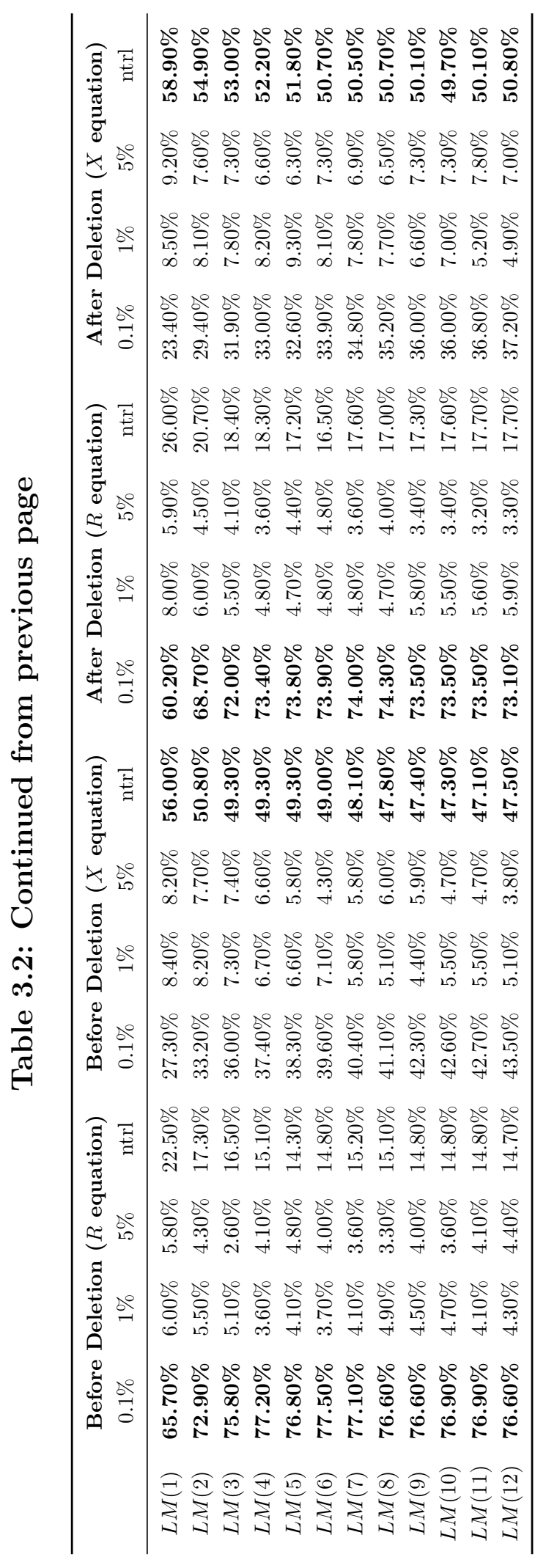




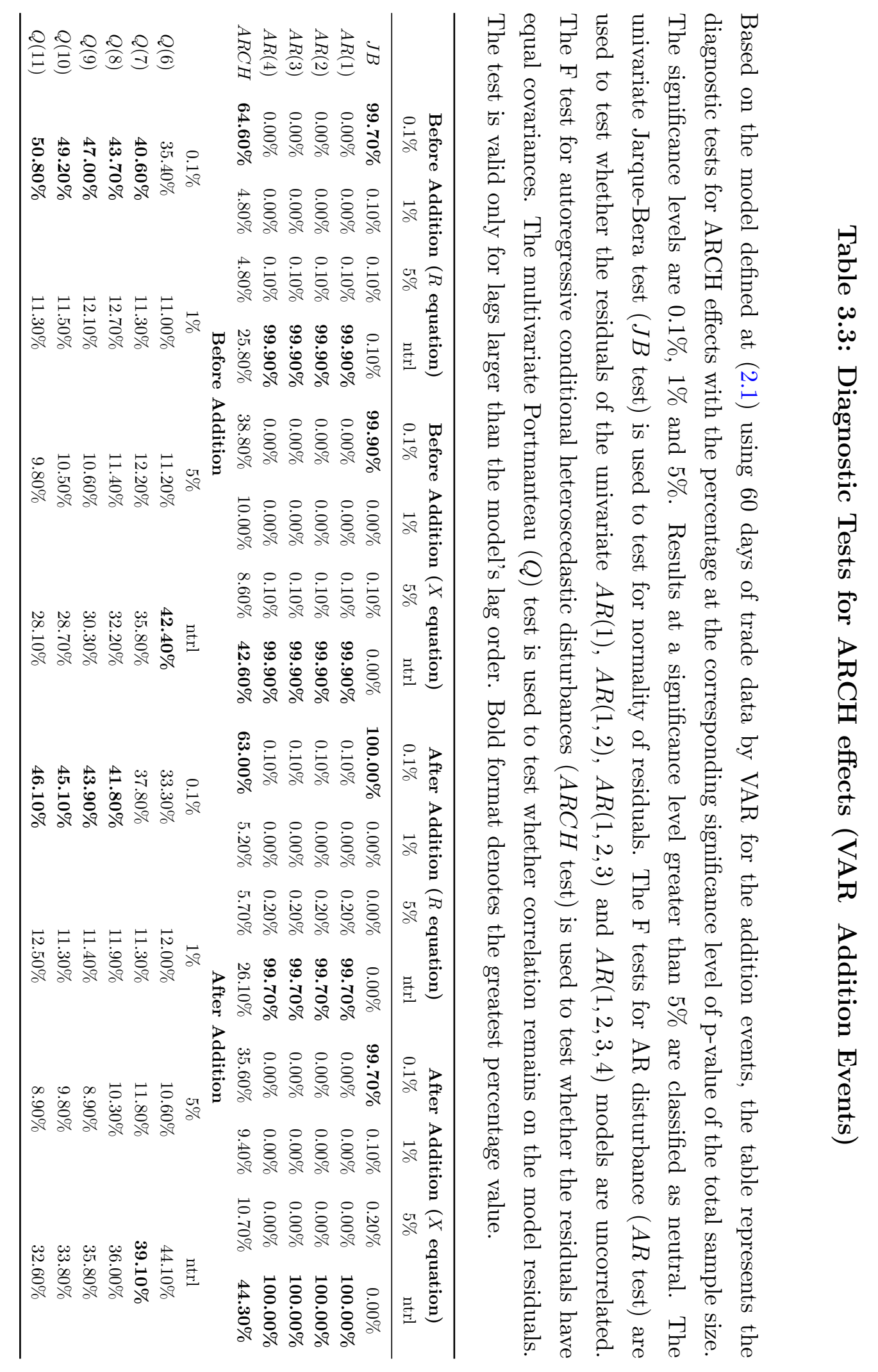




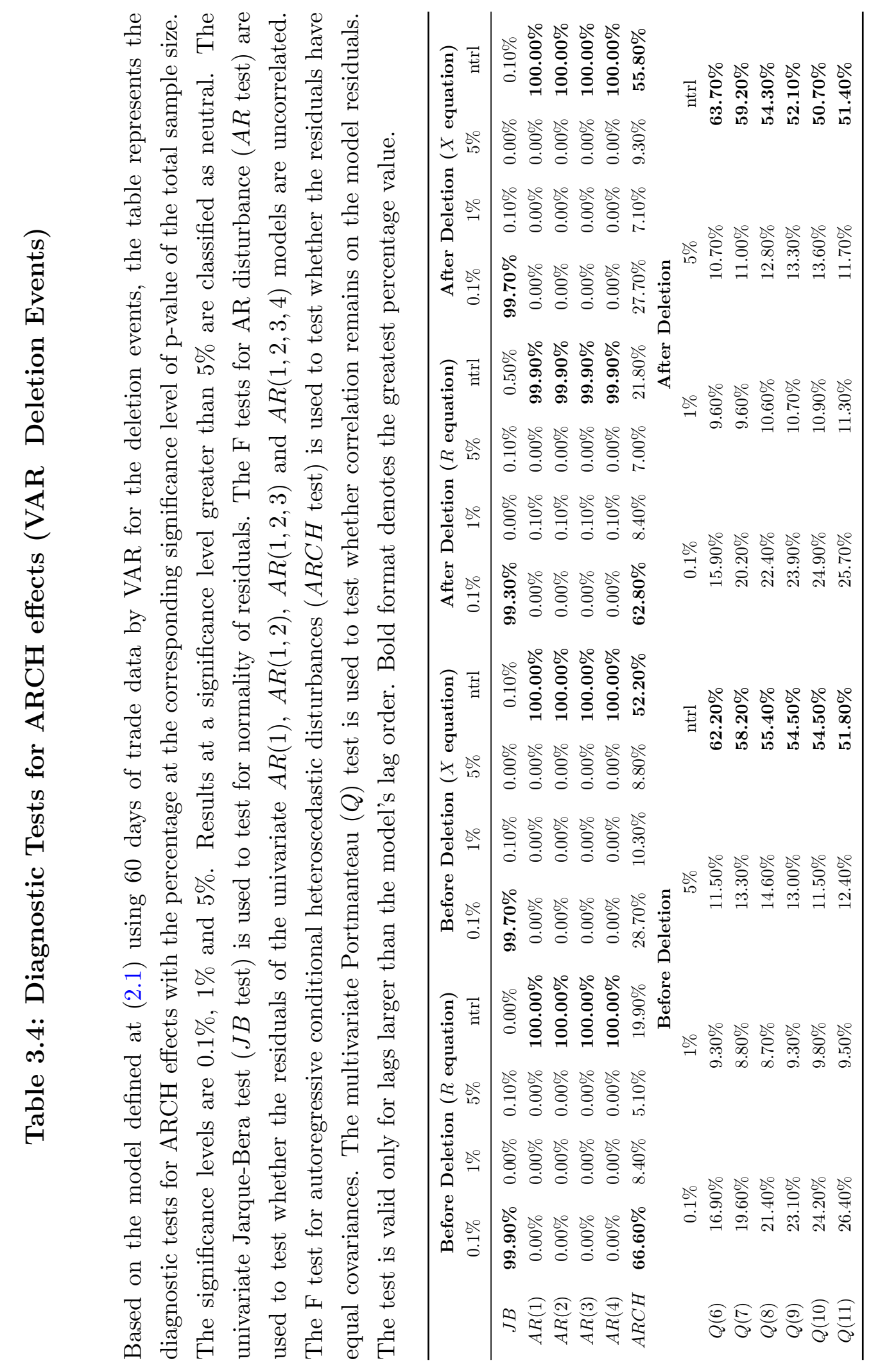




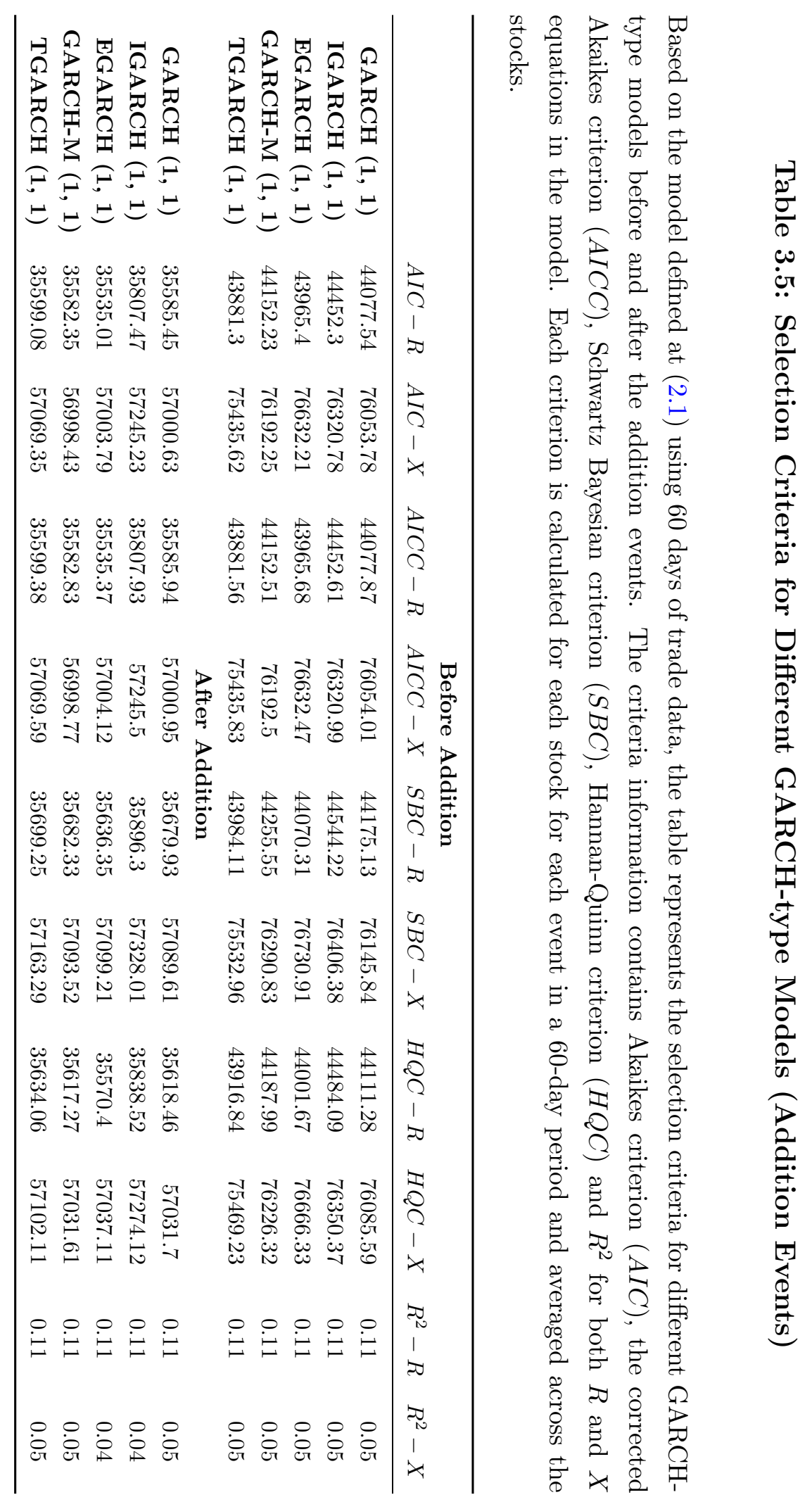




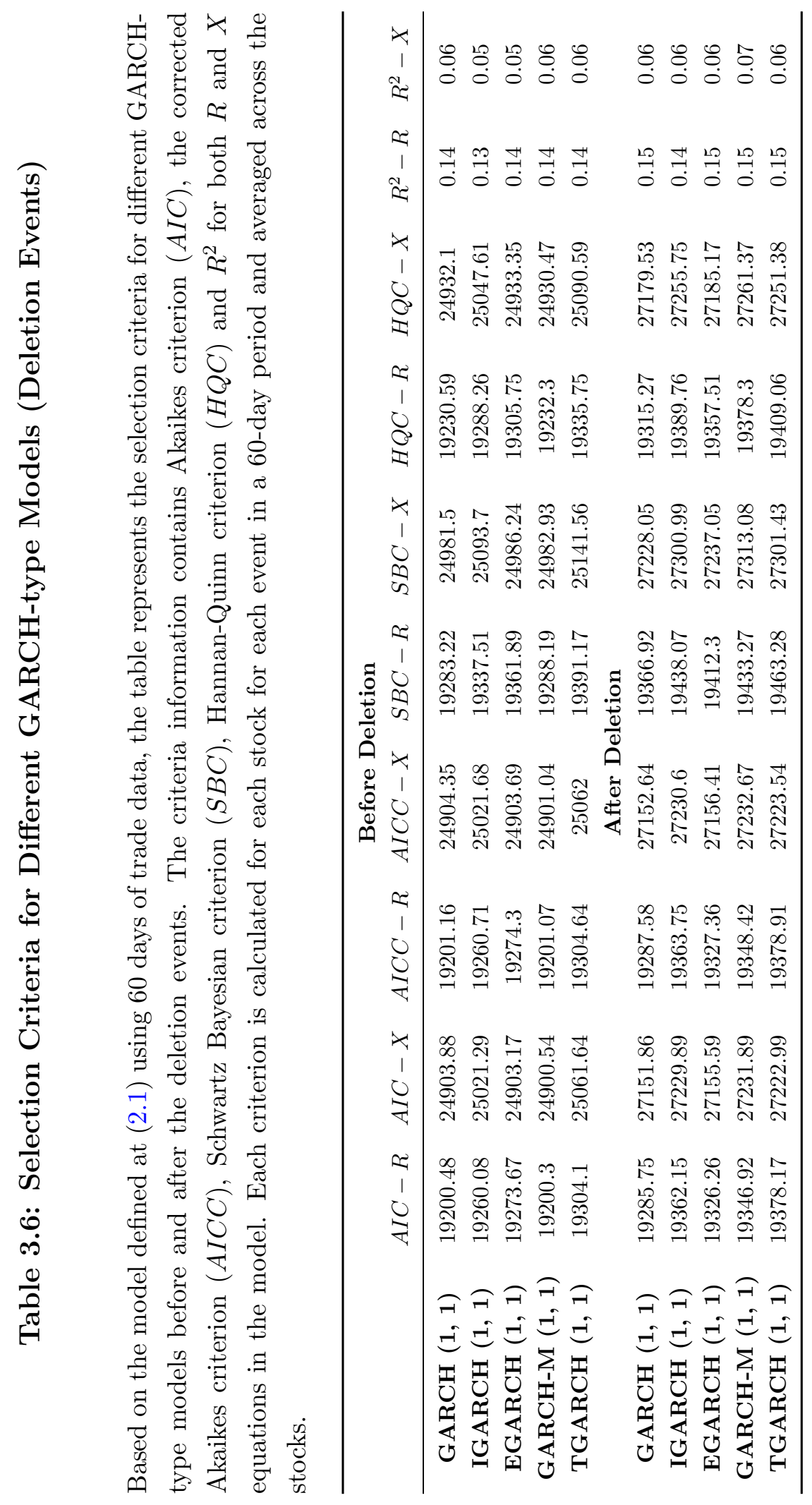




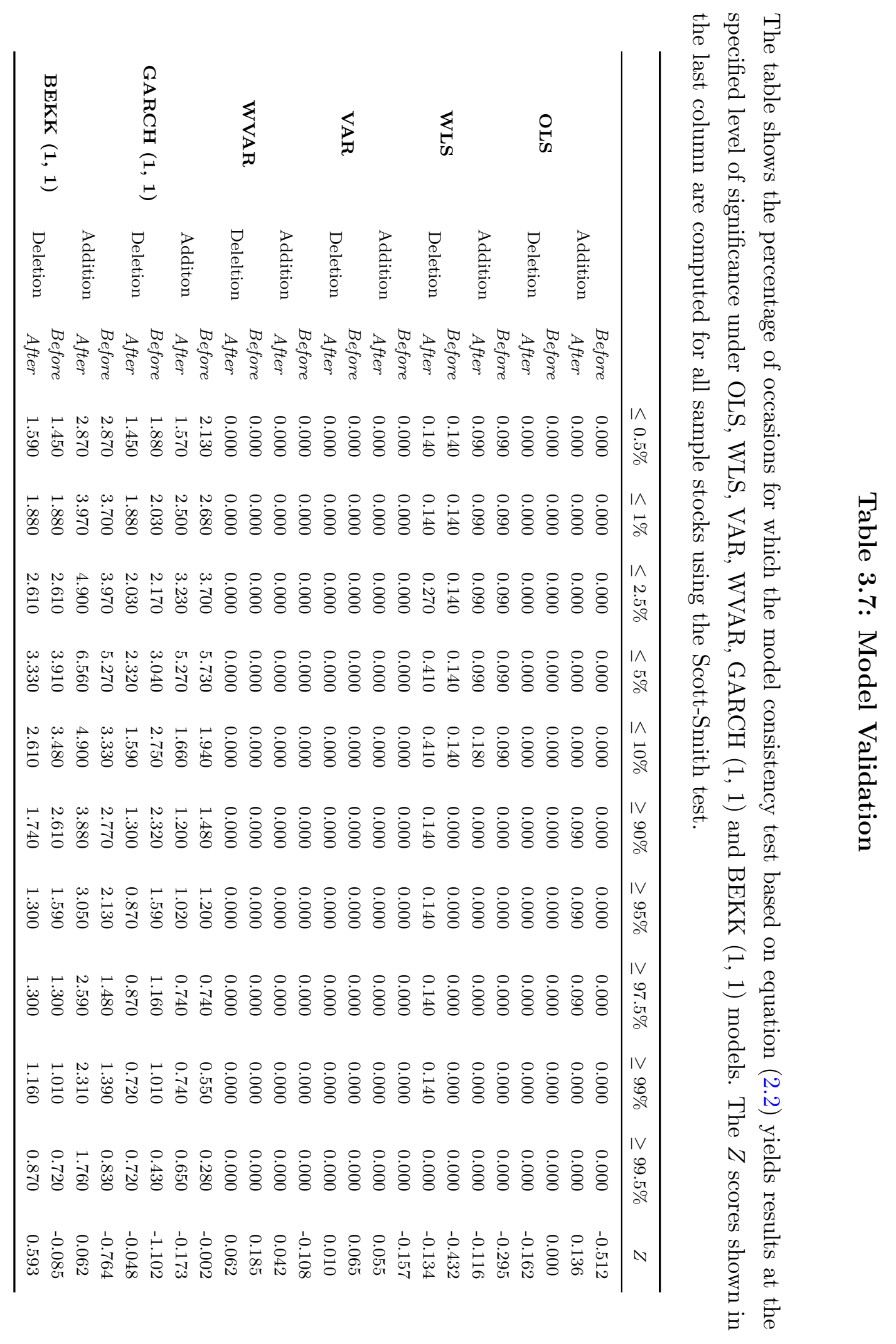




\section{Table 3.8: Frequency of Changes in Model Parameters for Addition Events (GARCH)}

Based on the model defined at (3.1) using 60 days of trade data by GARCH $(1,1)$. The first panel shows results for individual parameters. The second panel shows results for the sums of the $\alpha \mathrm{s}, \beta \mathrm{s}, \gamma \mathrm{s}$ and $\delta \mathrm{s}$ respectively. The first vertical section show the numbers of stocks for which the corresponding parameter is significantly less than zero, neutral or significantly greater than zero before the addition event. The significance level is defined at the $1 \%$. The second vertical section shows the corresponding results after the addition event. The section headed "Chsq" shows the value of a Chi-squared statistic that is used to test the difference between the observed frequencies in each row. The last section shows the results for the differences in parameters during the addition events and it shows the numbers of stocks for which the corresponding parameter exhibits significant decrease, neutral change or significant increase in its magnitude during the addition events. Differences are calculated by absolute value of model parameters between after and before the addition events. Cells in which there is a substantial number of statistically significant changes (more than $10 \%$ of the sample size, 1,082) are shown in bold.

\begin{tabular}{ccccccccccc}
\hline \multicolumn{6}{c}{ Before Addition } & \multicolumn{2}{c}{ After Addition } & \multicolumn{2}{c}{ Chsq } & \multicolumn{2}{c}{ Difference (After minus Before) } \\
& -ive & ntrl & + ve & -ive & ntrl & +ve & 2 DoF & sig. dec. & no sig. change & sig. inc. \\
\hline$\alpha_{0}$ & 149 & 869 & 64 & 146 & 870 & 66 & 0.970 & 40 & 999 & 43 \\
$\alpha_{1}$ & 1032 & 50 & 0 & 1031 & 51 & 0 & 0.995 & $\mathbf{1 8 8}$ & 716 & $\mathbf{1 7 8}$ \\
$\alpha_{2}$ & 994 & 86 & 2 & 982 & 97 & 3 & 0.627 & $\mathbf{1 6 2}$ & 795 & $\mathbf{1 2 5}$ \\
$\alpha_{3}$ & 849 & 222 & 11 & 811 & 253 & 18 & 0.101 & 107 & 868 & 107 \\
$\alpha_{4}$ & 666 & 389 & 27 & 601 & 446 & 35 & 0.016 & 85 & 912 & 85 \\
$\alpha_{5}$ & 468 & 573 & 41 & 463 & 578 & 41 & 0.976 & 63 & 943 & 76 \\
$\beta_{0}$ & 4 & 59 & 1019 & 2 & 65 & 1015 & 0.617 & $\mathbf{3 4 4}$ & 318 & $\mathbf{4 2 0}$ \\
$\beta_{1}$ & 3 & 107 & 972 & 5 & 136 & 941 & 0.107 & $\mathbf{2 0 5}$ & 550 & $\mathbf{3 2 7}$ \\
$\beta_{2}$ & 10 & 265 & 807 & 1 & 334 & 747 & 0.000 & 106 & 784 & $\mathbf{1 9 2}$ \\
$\beta_{3}$ & 10 & 474 & 598 & 9 & 516 & 557 & 0.193 & 58 & 899 & $\mathbf{1 2 5}$ \\
$\beta_{4}$ & 16 & 654 & 412 & 14 & 705 & 363 & 0.076 & 42 & 956 & 84 \\
$\beta_{5}$ & 22 & 772 & 288 & 20 & 794 & 268 & 0.570 & 31 & 986 & 65 \\
$\gamma_{0}$ & 225 & 745 & 112 & 207 & 758 & 117 & 0.615 & 58 & 952 & 72 \\
$\gamma_{1}$ & 267 & 674 & 141 & 281 & 692 & 109 & 0.096 & 98 & 914 & 70 \\
$\gamma_{2}$ & 187 & 817 & 78 & 169 & 853 & 60 & 0.133 & 57 & 993 & 32 \\
$\gamma_{3}$ & 88 & 916 & 78 & 113 & 908 & 61 & 0.073 & 34 & 1018 & 30 \\
$\gamma_{4}$ & 65 & 958 & 59 & 75 & 950 & 57 & 0.676 & 29 & 1028 & 25 \\
$\gamma_{5}$ & 52 & 948 & 82 & 61 & 968 & 53 & 0.028 & 28 & 1032 & 22 \\
$\delta_{1}$ & 7 & 64 & 1011 & 8 & 95 & 979 & 0.036 & $\mathbf{1 4 7}$ & 810 & $\mathbf{1 2 5}$ \\
$\delta_{2}$ & 11 & 179 & 892 & 18 & 242 & 822 & 0.001 & 90 & 905 & 87 \\
$\delta_{3}$ & 13 & 338 & 731 & 29 & 388 & 665 & 0.002 & 87 & 919 & 76 \\
$\delta_{4}$ & 15 & 467 & 600 & 26 & 477 & 579 & 0.180 & 58 & 951 & 73 \\
$\delta_{5}$ & 19 & 529 & 534 & 29 & 558 & 495 & 0.114 & 70 & 939 & 73 \\
$A$ & 1038 & 42 & 2 & 1040 & 41 & 1 & 0.841 & $\mathbf{2 1 0}$ & 687 & $\mathbf{1 8 5}$ \\
$B$ & 1 & 39 & 1042 & 0 & 59 & 1023 & 0.072 & $\mathbf{2 9 0}$ & 385 & $\mathbf{4 0 7}$ \\
$\Gamma$ & 189 & 767 & 126 & 217 & 774 & 91 & 0.022 & 77 & 955 & 50 \\
$\Delta$ & 7 & 39 & 1036 & 11 & 44 & 1027 & 0.541 & $\mathbf{1 8 2}$ & 762 & $\mathbf{1 3 8}$ \\
\hline & & & & & & & & & & \\
\hline
\end{tabular}


Table 3.9: Frequency of Changes in Model Parameters for Deletion Events (GARCH)

Based on the model defined at (3.1) using 60 days of trade data by $\operatorname{GARCH}(1,1)$. The first panel shows results for individual parameters. The second panel shows results for the sums of the $\alpha \mathrm{s}, \beta \mathrm{s}, \gamma \mathrm{s}$ and $\delta \mathrm{s}$ respectively. The first vertical section show the numbers of stocks for which the corresponding parameter is significantly less than zero, neutral or significantly greater than zero before the deletion event. The significance level is defined at the $1 \%$. The second vertical section shows the corresponding results after the deletion event. The section headed "Chsq" shows the value of a Chi-squared statistic that is used to test the difference between the observed frequencies in each row. The last section shows the results for the differences in parameters during the deletion events and it shows the numbers of stocks for which the corresponding parameter exhibits significant decrease, neutral change or significant increase in its magnitude during the deletion events. Differences are calculated by absolute value of model parameters between after and before the deletion events. Cells in which there is a substantial number of statistically significant changes (more than $10 \%$ of the sample size, 690) are shown in bold.

\begin{tabular}{ccccccccccc}
\hline \multicolumn{6}{c}{ Before Deletion } & \multicolumn{2}{c}{ After Deletion } & \multicolumn{2}{c}{ Chsq } & \multicolumn{2}{c}{ Difference (After minus Before) } \\
& -ive & ntrl & + +ve & -ive & ntrl & +ve & 2 DoF & sig. dec. & no sig. change & sig. inc. \\
\hline$\alpha_{0}$ & 63 & 573 & 54 & 61 & 572 & 57 & 0.944 & 25 & 649 & 16 \\
$\alpha_{1}$ & 653 & 37 & 0 & 641 & 49 & 0 & 0.409 & $\mathbf{8 5}$ & 484 & $\mathbf{1 2 1}$ \\
$\alpha_{2}$ & 597 & 90 & 3 & 581 & 107 & 2 & 0.390 & $\mathbf{7 6}$ & 539 & $\mathbf{7 5}$ \\
$\alpha_{3}$ & 488 & 196 & 6 & 460 & 220 & 10 & 0.201 & 61 & 571 & 58 \\
$\alpha_{4}$ & 323 & 349 & 18 & 339 & 335 & 16 & 0.673 & 35 & 619 & 36 \\
$\alpha_{5}$ & 238 & 427 & 25 & 240 & 428 & 22 & 0.904 & 36 & 613 & 41 \\
$\beta_{0}$ & 2 & 41 & 647 & 1 & 54 & 635 & 0.329 & $\mathbf{2 7 5}$ & 254 & $\mathbf{1 6 1}$ \\
$\beta_{1}$ & 5 & 161 & 524 & 5 & 188 & 497 & 0.246 & $\mathbf{1 3 0}$ & 491 & 69 \\
$\beta_{2}$ & 7 & 354 & 329 & 9 & 351 & 330 & 0.876 & 65 & 589 & 36 \\
$\beta_{3}$ & 11 & 471 & 208 & 8 & 459 & 223 & 0.563 & 32 & 634 & 24 \\
$\beta_{4}$ & 16 & 571 & 103 & 17 & 562 & 111 & 0.818 & 23 & 652 & 15 \\
$\beta_{5}$ & 22 & 616 & 52 & 23 & 602 & 65 & 0.443 & 18 & 657 & 15 \\
$\gamma_{0}$ & 118 & 514 & 58 & 87 & 543 & 60 & 0.063 & 34 & 625 & 31 \\
$\gamma_{1}$ & 186 & 467 & 37 & 204 & 464 & 22 & 0.098 & 33 & 580 & $\mathbf{7 7}$ \\
$\gamma_{2}$ & 95 & 575 & 20 & 123 & 549 & 18 & 0.116 & 10 & 642 & 38 \\
$\gamma_{3}$ & 65 & 603 & 22 & 80 & 588 & 22 & 0.419 & 15 & 640 & 35 \\
$\gamma_{4}$ & 47 & 618 & 25 & 47 & 622 & 21 & 0.835 & 11 & 658 & 21 \\
$\gamma_{5}$ & 36 & 626 & 28 & 43 & 622 & 25 & 0.669 & 11 & 658 & 21 \\
$\delta_{1}$ & 11 & 130 & 549 & 5 & 141 & 544 & 0.257 & 66 & 556 & 68 \\
$\delta_{2}$ & 16 & 265 & 409 & 13 & 269 & 408 & 0.843 & 27 & 618 & 45 \\
$\delta_{3}$ & 13 & 412 & 265 & 13 & 376 & 301 & 0.140 & 23 & 632 & 35 \\
$\delta_{4}$ & 23 & 445 & 222 & 10 & 450 & 230 & 0.071 & 30 & 626 & 34 \\
$\delta_{5}$ & 22 & 476 & 192 & 16 & 475 & 199 & 0.585 & 28 & 633 & 29 \\
$A$ & 642 & 46 & 2 & 620 & 69 & 1 & 0.070 & $\mathbf{9 4}$ & 485 & $\mathbf{1 1 1}$ \\
$B$ & 1 & 54 & 635 & 1 & 77 & 612 & 0.107 & $\mathbf{2 0 8}$ & 353 & $\mathbf{1 2 9}$ \\
$\Gamma$ & 121 & 547 & 22 & 155 & 516 & 19 & 0.070 & 19 & 610 & 61 \\
$\Delta$ & 7 & 76 & 607 & 2 & 91 & 597 & 0.122 & 69 & 528 & $\mathbf{9 3}$ \\
\hline & & & & & & & & & & \\
\end{tabular}




\section{Table 3.10: Frequency of Changes in Model Parameters for Addition Events (BEKK)}

Based on the model defined at (3.2) using 60 days of trade data by BEKK $(1,1)$. The first panel shows results for individual parameters. The second panel shows results for the sums of the $\alpha \mathrm{s}, \beta \mathrm{s}, \gamma \mathrm{s}$ and $\delta \mathrm{s}$ respectively. The first vertical section show the numbers of stocks for which the corresponding parameter is significantly less than zero, neutral or significantly greater than zero before the addition event. The significance level is defined at the 1\%. The second vertical section shows the corresponding results after the addition event. The section headed "Chsq" shows the value of a Chi-squared statistic that is used to test the difference between the observed frequencies in each row. The last section shows the results for the differences in parameters during the addition events and it shows the numbers of stocks for which the corresponding parameter exhibits significant decrease, neutral change or significant increase in its magnitude during the addition events. Differences are calculated by absolute value of model parameters between after and before the addition events. Cells in which there is a substantial number of statistically significant changes (more than $10 \%$ of the sample size, 1,082) are shown in bold.

\begin{tabular}{ccccccccccc}
\hline \multicolumn{6}{c}{ Before Addition } & \multicolumn{2}{c}{ After Addition } & \multicolumn{2}{c}{ Chsq } & \multicolumn{2}{c}{ Difference (After minus Before) } \\
& -ive & ntrl & + ve & -ive & ntrl & +ve & 2 DoF & sig. dec. & no sig. change & sig. inc. \\
\hline$\alpha_{0}$ & 133 & 887 & 62 & 140 & 873 & 69 & 0.717 & 29 & 999 & 54 \\
$\alpha_{1}$ & 1044 & 36 & 2 & 1030 & 49 & 3 & 0.319 & $\mathbf{1 9 5}$ & 716 & $\mathbf{1 7 1}$ \\
$\alpha_{2}$ & 940 & 137 & 5 & 914 & 163 & 5 & 0.270 & $\mathbf{1 5 1}$ & 834 & 97 \\
$\alpha_{3}$ & 752 & 321 & 9 & 700 & 365 & 17 & 0.028 & 100 & 903 & 79 \\
$\alpha_{4}$ & 554 & 518 & 10 & 487 & 576 & 19 & 0.006 & 81 & 938 & 63 \\
$\alpha_{5}$ & 381 & 681 & 20 & 324 & 716 & 42 & 0.001 & 61 & 972 & 49 \\
$\beta_{0}$ & 16 & 48 & 1018 & 13 & 57 & 1012 & 0.577 & $\mathbf{3 0 5}$ & 374 & $\mathbf{4 0 3}$ \\
$\beta_{1}$ & 168 & 155 & 759 & 149 & 194 & 739 & 0.056 & $\mathbf{2 7 1}$ & 594 & $\mathbf{2 1 7}$ \\
$\beta_{2}$ & 172 & 330 & 580 & 138 & 390 & 554 & 0.009 & $\mathbf{1 7 9}$ & 775 & $\mathbf{1 2 8}$ \\
$\beta_{3}$ & 171 & 520 & 391 & 157 & 551 & 374 & 0.392 & $\mathbf{1 5 4}$ & 830 & 98 \\
$\beta_{4}$ & 181 & 670 & 231 & 158 & 688 & 236 & 0.396 & $\mathbf{1 4 2}$ & 886 & 54 \\
$\beta_{5}$ & 184 & 731 & 167 & 166 & 747 & 169 & 0.574 & $\mathbf{1 4 0}$ & 892 & 50 \\
$\gamma_{0}$ & 212 & 695 & 175 & 213 & 701 & 168 & 0.918 & 77 & 918 & 87 \\
$\gamma_{1}$ & 311 & 625 & 146 & 321 & 652 & 109 & 0.047 & 108 & 890 & 84 \\
$\gamma_{2}$ & 223 & 787 & 72 & 221 & 790 & 71 & 0.989 & 70 & 954 & 58 \\
$\gamma_{3}$ & 139 & 872 & 71 & 165 & 850 & 67 & 0.270 & 50 & 991 & 41 \\
$\gamma_{4}$ & 114 & 897 & 71 & 115 & 893 & 74 & 0.963 & 55 & 993 & 34 \\
$\gamma_{5}$ & 69 & 930 & 83 & 82 & 933 & 67 & 0.243 & 44 & 997 & 41 \\
$\delta_{1}$ & 45 & 84 & 953 & 38 & 94 & 950 & 0.561 & $\mathbf{2 1 6}$ & 735 & $\mathbf{1 3 1}$ \\
$\delta_{2}$ & 37 & 208 & 837 & 47 & 256 & 779 & 0.016 & $\mathbf{1 4 0}$ & 852 & 90 \\
$\delta_{3}$ & 54 & 372 & 656 & 53 & 414 & 615 & 0.167 & $\mathbf{1 3 9}$ & 862 & 81 \\
$\delta_{4}$ & 55 & 493 & 534 & 54 & 532 & 496 & 0.235 & $\mathbf{1 2 3}$ & 886 & 73 \\
$\delta_{5}$ & 61 & 559 & 462 & 59 & 605 & 418 & 0.132 & 103 & 901 & 78 \\
$A$ & 1027 & 54 & 1 & 1005 & 73 & 4 & 0.087 & $\mathbf{2 1 3}$ & 732 & $\mathbf{1 3 7}$ \\
$B$ & 13 & 42 & 1027 & 11 & 38 & 1033 & 0.825 & $\mathbf{2 6 5}$ & 423 & $\mathbf{3 9 4}$ \\
$\Gamma$ & 255 & 696 & 131 & 264 & 718 & 100 & 0.097 & 99 & 912 & 71 \\
$\Delta$ & 29 & 30 & 1023 & 25 & 47 & 1010 & 0.127 & $\mathbf{2 2 7}$ & 684 & $\mathbf{1 7 1}$ \\
\hline & & & & & & & & & & \\
\end{tabular}




\section{Table 3.11: Frequency of Changes in Model Parameters for Deletion Events (BEKK)}

Based on the model defined at (3.2) using 60 days of trade data by BEKK $(1,1)$. The first panel shows results for individual parameters. The second panel shows results for the sums of the $\alpha \mathrm{s}, \beta \mathrm{s}, \gamma \mathrm{s}$ and $\delta \mathrm{s}$ respectively. The first vertical section show the numbers of stocks for which the corresponding parameter is significantly less than zero, neutral or significantly greater than zero before the deletion event. The significance level is defined at the $1 \%$. The second vertical section shows the corresponding results after the deletion event. The section headed "Chsq" shows the value of a Chi-squared statistic that is used to test the difference between the observed frequencies in each row. The last section shows the results for the differences in parameters during the deletion events and it shows the numbers of stocks for which the corresponding parameter exhibits significant decrease, neutral change or significant increase in its magnitude during the deletion events. Differences are calculated by absolute value of model parameters between after and before the deletion events. Cells in which there is a substantial number of statistically significant changes (more than $10 \%$ of the sample size, 690) are shown in bold.

\begin{tabular}{|c|c|c|c|c|c|c|c|c|c|c|}
\hline & \multicolumn{3}{|c|}{ Before Deletion } & \multicolumn{3}{|c|}{ After Deletion } & \multirow{2}{*}{$\begin{array}{l}\text { Chsq } \\
2 \text { DoF }\end{array}$} & \multicolumn{2}{|c|}{ Difference (After minus } & \multirow{2}{*}{$\begin{array}{l}\text { Before) } \\
\text { sig. inc. }\end{array}$} \\
\hline & -ive & ntrl & $+\mathrm{ve}$ & -ive & ntrl & $+\mathrm{ve}$ & & sig. dec. & no sig. change & \\
\hline$\alpha_{0}$ & 75 & 571 & 44 & 66 & 587 & 37 & 0.496 & 25 & 654 & 11 \\
\hline$\alpha_{1}$ & 644 & 43 & 3 & 643 & 46 & 1 & 0.576 & 88 & 507 & 95 \\
\hline$\alpha_{2}$ & 544 & 139 & 7 & 526 & 162 & 2 & 0.089 & 70 & 563 & 57 \\
\hline$\alpha_{3}$ & 395 & 288 & 7 & 398 & 288 & 4 & 0.660 & 56 & 593 & 41 \\
\hline$\alpha_{4}$ & 240 & 438 & 12 & 277 & 404 & 9 & 0.108 & 36 & 622 & 32 \\
\hline$\alpha_{5}$ & 170 & 506 & 14 & 192 & 489 & 9 & 0.257 & 37 & 630 & 23 \\
\hline$\beta_{0}$ & 3 & 40 & 647 & 10 & 42 & 638 & 0.144 & 262 & 266 & 162 \\
\hline$\beta_{1}$ & 83 & 213 & 394 & 90 & 210 & 390 & 0.850 & 135 & 504 & 51 \\
\hline$\beta_{2}$ & 80 & 381 & 229 & 95 & 368 & 227 & 0.468 & 93 & 573 & 24 \\
\hline$\beta_{3}$ & 88 & 470 & 132 & 93 & 468 & 129 & 0.915 & 85 & 590 & 15 \\
\hline$\beta_{4}$ & 97 & 539 & 54 & 101 & 523 & 66 & 0.467 & 77 & 600 & 13 \\
\hline$\beta_{5}$ & 94 & 561 & 35 & 104 & 560 & 26 & 0.400 & 77 & 603 & 10 \\
\hline$\gamma_{0}$ & 156 & 453 & 81 & 119 & 503 & 68 & 0.013 & 40 & 605 & 45 \\
\hline$\gamma_{1}$ & 235 & 424 & 31 & 248 & 418 & 24 & 0.526 & 39 & 589 & 62 \\
\hline$\gamma_{2}$ & 137 & 529 & 24 & 159 & 511 & 20 & 0.315 & 24 & 627 & 39 \\
\hline$\gamma_{3}$ & 102 & 570 & 18 & 113 & 563 & 14 & 0.575 & 24 & 633 & 33 \\
\hline$\gamma_{4}$ & 95 & 579 & 16 & 71 & 597 & 22 & 0.096 & 24 & 648 & 18 \\
\hline$\gamma_{5}$ & 75 & 585 & 30 & 70 & 597 & 23 & 0.544 & 28 & 642 & 20 \\
\hline$\delta_{1}$ & 33 & 106 & 551 & 41 & 119 & 530 & 0.364 & 81 & 536 & 73 \\
\hline$\delta_{2}$ & 37 & 262 & 391 & 43 & 267 & 380 & 0.721 & 56 & 585 & 49 \\
\hline$\delta_{3}$ & 44 & 379 & 267 & 47 & 373 & 270 & 0.921 & 50 & 606 & 34 \\
\hline$\delta_{4}$ & 48 & 446 & 196 & 46 & 446 & 198 & 0.974 & 60 & 604 & 26 \\
\hline$\delta_{5}$ & 52 & 485 & 153 & 55 & 482 & 153 & 0.954 & 50 & 616 & 24 \\
\hline$A$ & 616 & 72 & 2 & 600 & 86 & 4 & 0.347 & 87 & 515 & 88 \\
\hline$B$ & 3 & 50 & 637 & 8 & 60 & 622 & 0.186 & 207 & 349 & 134 \\
\hline$\Gamma$ & 190 & 472 & 28 & 191 & 471 & 28 & 0.998 & 39 & 597 & 54 \\
\hline$\Delta$ & 29 & 65 & 596 & 31 & 71 & 588 & 0.825 & 88 & 507 & 95 \\
\hline
\end{tabular}




\section{Table 3.12: Comparison on Frequency of Changes in Model Parameters for Addition Events}

The table reports a comparison of the summary results for the sums of the $\alpha \mathrm{s}, \beta \mathrm{s}, \gamma \mathrm{s}$ and $\delta \mathrm{s}$ under GARCH $(1,1)$ and BEKK $(1,1)$ with the corresponding tables under OLS and VAR for the addition events. The first vertical section show the percentage of stocks for which the corresponding parameter is significantly less than zero, neutral or significantly greater than zero before the addition events. The significance level is defined at the $1 \%$. The second vertical section shows the corresponding results after addition event. The third section shows the results for the differences in parameters during the addition events and it shows the numbers of stocks for which the corresponding parameter exhibits significant decrease, neutral change or significant increase in its magnitude during the addition events. Differences are calculated by absolute value of model parameters between after and before the addition events. The last column shows the size of data sets used under each model.

\begin{tabular}{|c|c|c|c|c|c|c|c|c|c|c|}
\hline \multicolumn{11}{|c|}{ Addition (\%) } \\
\hline & -ive & $\begin{array}{c}\text { Before } \\
\text { ntrl }\end{array}$ & $+\mathrm{ve}$ & -ive & $\begin{array}{l}\text { After } \\
\text { ntrl }\end{array}$ & $+\mathrm{ve}$ & $\begin{array}{c}\text { Differe } \\
\text { sig. dec. }\end{array}$ & $\begin{array}{l}\text { ce (After minus } \\
\text { no sig. change }\end{array}$ & $\begin{array}{l}\text { Before) } \\
\text { sig. inc. }\end{array}$ & Sample Size \\
\hline \multicolumn{11}{|c|}{ OLS } \\
\hline$A$ & 90.77 & 9.14 & 0.09 & 89.26 & 10.74 & 0.00 & 7.90 & 84.29 & 7.81 & 1,127 \\
\hline$B$ & 0.27 & 4.61 & 95.12 & 0.09 & 5.77 & 94.14 & 18.19 & 52.26 & 29.55 & 1,127 \\
\hline$\Gamma$ & 16.50 & 72.40 & 11.09 & 16.50 & 76.40 & 7.10 & 5.06 & 92.10 & 2.84 & 1,127 \\
\hline$\Delta$ & 0.00 & 6.39 & 93.61 & 0.09 & 7.72 & 92.19 & 5.68 & 90.42 & 3.90 & 1,127 \\
\hline \multicolumn{11}{|c|}{ VAR } \\
\hline$A$ & 94.85 & 5.15 & 0.00 & 94.06 & 5.86 & 0.09 & 15.44 & 69.03 & 15.53 & 1,127 \\
\hline$B$ & 0.09 & 5.32 & 94.59 & 0.00 & 5.77 & 94.23 & 18.46 & 52.97 & 28.57 & 1,127 \\
\hline$\Gamma$ & 15.97 & 72.76 & 11.27 & 15.17 & 77.82 & 7.01 & 6.21 & 90.68 & 3.11 & 1,127 \\
\hline$\Delta$ & 0.00 & 3.37 & 96.63 & 0.00 & 4.61 & 95.39 & 16.06 & 73.03 & 10.91 & 1,127 \\
\hline \multicolumn{11}{|c|}{ GARCH } \\
\hline$A$ & 95.93 & 3.88 & 0.18 & 96.12 & 3.79 & 0.09 & 19.41 & 63.49 & 17.10 & 1,082 \\
\hline$B$ & 0.09 & 3.60 & 96.30 & 0.00 & 5.45 & 94.55 & 26.80 & 35.58 & 37.62 & 1,082 \\
\hline$\Gamma$ & 17.47 & 70.89 & 11.65 & 20.06 & 71.53 & 8.41 & 7.12 & 88.26 & 4.62 & 1,082 \\
\hline$\Delta$ & 0.65 & 3.60 & 95.75 & 1.02 & 4.07 & 94.92 & 16.82 & 70.43 & 12.75 & 1,082 \\
\hline \multicolumn{11}{|c|}{ BEKK } \\
\hline$A$ & 94.92 & 4.99 & 0.09 & 92.88 & 6.75 & 0.37 & 19.69 & 67.65 & 12.66 & 1,082 \\
\hline$B$ & 1.20 & 3.88 & 94.92 & 1.02 & 3.51 & 95.47 & 24.49 & 39.09 & 36.41 & 1,082 \\
\hline$\Gamma$ & 23.57 & 64.33 & 12.11 & 24.40 & 66.36 & 9.24 & 9.15 & 84.29 & 6.56 & 1,082 \\
\hline$\Delta$ & 2.68 & 2.77 & 94.55 & 2.31 & 4.34 & 93.35 & 20.98 & 63.22 & 15.80 & 1,082 \\
\hline
\end{tabular}




\section{Table 3.13: Comparison on Frequency of Changes in Model Parameters for Deletion Events}

The table reports a comparison of the summary results for the sums of the $\alpha \mathrm{s}, \beta \mathrm{s}, \gamma \mathrm{s}$ and $\delta$ s under GARCH (1, $1)$ and $\operatorname{BEKK}(1,1)$ with the corresponding tables under OLS and VAR for the deletion events. The first vertical section show the percentage of stocks for which the corresponding parameter is significantly less than zero, neutral or significantly greater than zero before the deletion events. The significance level is defined at the $1 \%$. The second vertical section shows the corresponding results after deletion event. The third section shows the results for the differences in parameters during the deletion events and it shows the numbers of stocks for which the corresponding parameter exhibits significant decrease, neutral change or significant increase in its magnitude during the deletion events. Differences are calculated by absolute value of model parameters between after and before the deletion events. The last column shows the size of data sets used under each model.

\begin{tabular}{|c|c|c|c|c|c|c|c|c|c|c|}
\hline \multicolumn{11}{|c|}{ Deletion (\%) } \\
\hline & & Before & & & After & & Differe & ce (After minus & Before) & \\
\hline & -ive & ntrl & $+\mathrm{ve}$ & -ive & ntrl & + ve & sig. dec. & no sig. change & sig. inc. & Sample Size \\
\hline \multicolumn{11}{|c|}{ OLS } \\
\hline$A$ & 81.59 & 18.41 & 0.00 & 83.38 & 16.62 & 0.00 & 3.57 & 88.46 & 7.97 & 728 \\
\hline$B$ & 0.00 & 8.79 & 91.21 & 0.00 & 15.25 & 84.75 & 21.70 & 65.80 & 12.50 & 728 \\
\hline$\Gamma$ & 17.86 & 79.53 & 2.61 & 19.37 & 78.57 & 2.06 & 2.20 & 93.54 & 4.26 & 728 \\
\hline$\Delta$ & 0.00 & 15.80 & 84.20 & 0.14 & 17.72 & 82.14 & 2.47 & 93.13 & 4.40 & 728 \\
\hline \multicolumn{11}{|c|}{ VAR } \\
\hline$A$ & 90.38 & 9.48 & 0.14 & 90.38 & 9.62 & 0.00 & 10.30 & 72.12 & 17.58 & 728 \\
\hline$B$ & 0.00 & 8.93 & 91.07 & 0.00 & 13.19 & 86.81 & 21.84 & 65.52 & 12.64 & 728 \\
\hline$\Gamma$ & 15.93 & 81.04 & 3.02 & 17.17 & 80.36 & 2.47 & 2.20 & 93.82 & 3.98 & 728 \\
\hline$\Delta$ & 0.00 & 9.07 & 90.93 & 0.00 & 11.26 & 88.74 & 9.07 & 78.85 & 12.09 & 728 \\
\hline \multicolumn{11}{|c|}{ GARCH } \\
\hline$A$ & 93.04 & 6.67 & 0.29 & 89.86 & 10.00 & 0.14 & 13.62 & 70.29 & 16.09 & 690 \\
\hline$B$ & 0.14 & 7.83 & 92.03 & 0.14 & 11.16 & 88.70 & 30.14 & 51.16 & 18.70 & 690 \\
\hline$\Gamma$ & 17.54 & 79.28 & 3.19 & 22.46 & 74.78 & 2.75 & 2.75 & 88.41 & 8.84 & 690 \\
\hline$\Delta$ & 1.01 & 11.01 & 87.97 & 0.29 & 13.19 & 86.52 & 10.00 & 76.52 & 13.48 & 690 \\
\hline \multicolumn{11}{|c|}{ BEKK } \\
\hline$A$ & 89.28 & 10.43 & 0.29 & 86.96 & 12.46 & 0.58 & 12.61 & 74.64 & 12.75 & 690 \\
\hline$B$ & 0.43 & 7.25 & 92.32 & 1.16 & 8.70 & 90.14 & 30.00 & 50.58 & 19.42 & 690 \\
\hline$\Gamma$ & 27.54 & 68.41 & 4.06 & 27.68 & 68.26 & 4.06 & 5.65 & 86.52 & 7.83 & 690 \\
\hline$\Delta$ & 4.20 & 9.42 & 86.38 & 4.49 & 10.29 & 85.22 & 12.75 & 73.48 & 13.77 & 690 \\
\hline
\end{tabular}




\section{Table 3.14: Parameter Dynamics (GARCH)}

Based on the model defined at (3.1) using 60 days of trade data by GARCH $(1,1)$. Each panel shows the results of estimates of the sums of the $\alpha \mathrm{s}, \beta \mathrm{s}, \gamma \mathrm{s}$ and $\delta \mathrm{s}$ respectively. Rows of each panel are before the event and columns are after it. "N" and "P"denotes negative and positive respectively. All other changes at a significance level of which is greater than $5 \%$ are classified as neutral. Bold entries in the table are percentages which are substantial. More detailed tables which cover individual parameters are available on request.

\begin{tabular}{|c|c|c|c|c|c|c|c|c|c|c|}
\hline \multicolumn{6}{|c|}{ Addition } & \multicolumn{5}{|c|}{ Deletion } \\
\hline & $\mathrm{N}(1 \%)$ & $\mathrm{N}(5 \%)$ & Neutral & $\mathrm{P}(5 \%)$ & $\mathrm{P}(1 \%)$ & $\mathrm{N}(1 \%)$ & $\mathrm{N}(5 \%)$ & Neutral & $\mathrm{P}(5 \%)$ & $\mathrm{P}(1 \%)$ \\
\hline \multicolumn{11}{|c|}{$A$} \\
\hline $\mathrm{N}(1 \%)$ & 92.98 & 1.02 & 1.85 & 0.00 & 0.09 & 85.80 & 2.90 & 4.20 & 0.00 & 0.14 \\
\hline $\mathrm{N}(5 \%)$ & 0.74 & 0.09 & 0.37 & 0.00 & 0.00 & 0.72 & 0.00 & 0.58 & 0.00 & 0.00 \\
\hline Neutral & 2.22 & 0.09 & 0.28 & 0.09 & 0.00 & 3.04 & 0.87 & 1.45 & 0.00 & 0.00 \\
\hline $\mathrm{P}(5 \%)$ & 0.00 & 0.00 & 0.00 & 0.00 & 0.00 & 0.00 & 0.00 & 0.00 & 0.00 & 0.00 \\
\hline $\mathrm{P}(1 \%)$ & 0.18 & 0.00 & 0.00 & 0.00 & 0.00 & 0.29 & 0.00 & 0.00 & 0.00 & 0.00 \\
\hline \multicolumn{11}{|c|}{$B$} \\
\hline $\mathrm{N}(1 \%)$ & 0.00 & 0.00 & 0.09 & 0.00 & 0.00 & 0.00 & 0.00 & 0.00 & 0.14 & 0.00 \\
\hline $\mathrm{N}(5 \%)$ & 0.00 & 0.00 & 0.00 & 0.00 & 0.09 & 0.00 & 0.00 & 0.29 & 0.00 & 0.00 \\
\hline Neutral & 0.00 & 0.00 & 1.20 & 0.28 & 1.11 & 0.14 & 0.00 & 3.19 & 0.14 & 2.61 \\
\hline $\mathrm{P}(5 \%)$ & 0.00 & 0.00 & 0.09 & 0.09 & 0.74 & 0.00 & 0.00 & 0.29 & 0.29 & 0.87 \\
\hline $\mathrm{P}(1 \%)$ & 0.00 & 0.00 & 2.59 & 1.11 & 92.61 & 0.00 & 0.00 & 5.07 & 1.74 & 85.22 \\
\hline \multicolumn{11}{|c|}{$\Gamma$} \\
\hline $\mathrm{N}(1 \%)$ & 8.69 & 1.29 & 6.56 & 0.37 & 0.55 & 7.54 & 1.45 & 7.83 & 0.14 & 0.58 \\
\hline $\mathrm{N}(5 \%)$ & 1.94 & 0.74 & 3.51 & 0.00 & 0.37 & 3.33 & 0.58 & 5.36 & 0.00 & 0.29 \\
\hline Neutral & 8.13 & 3.51 & 44.64 & 1.85 & 3.14 & 10.87 & 4.93 & 47.10 & 2.03 & 1.59 \\
\hline $\mathrm{P}(5 \%)$ & 0.18 & 0.37 & 1.94 & 0.18 & 0.37 & 0.43 & 0.00 & 2.17 & 0.29 & 0.29 \\
\hline $\mathrm{P}(1 \%)$ & 1.11 & 0.55 & 5.45 & 0.55 & 3.97 & 0.29 & 0.14 & 2.46 & 0.29 & 0.00 \\
\hline \multicolumn{11}{|c|}{$\Delta$} \\
\hline $\mathrm{N}(1 \%)$ & 0.00 & 0.00 & 0.09 & 0.00 & 0.55 & 0.00 & 0.00 & 0.29 & 0.14 & 0.58 \\
\hline $\mathrm{N}(5 \%)$ & 0.00 & 0.00 & 0.00 & 0.00 & 0.18 & 0.00 & 0.00 & 0.00 & 0.00 & 0.00 \\
\hline Neutral & 0.18 & 0.00 & 0.18 & 0.28 & 1.48 & 0.00 & 0.00 & 1.88 & 0.43 & 4.64 \\
\hline $\mathrm{P}(5 \%)$ & 0.18 & 0.00 & 0.00 & 0.00 & 1.11 & 0.00 & 0.00 & 0.87 & 0.72 & 2.46 \\
\hline $\mathrm{P}(1 \%)$ & 0.65 & 0.00 & 2.59 & 0.92 & 91.59 & 0.29 & 0.00 & 6.23 & 2.61 & 78.84 \\
\hline
\end{tabular}




\section{Table 3.15: Parameter Dynamics (BEKK)}

Based on the model defined at (3.2) using 60 days of trade data by BEKK $(1,1)$. Each panel shows the results of estimates of the sums of the $\alpha \mathrm{s}, \beta \mathrm{s}, \gamma \mathrm{s}$ and $\delta$ s respectively. Rows of each panel are before the event and columns are after it. "N" and "P"denotes negative and positive respectively. All other changes at a significance level of which is greater than $5 \%$ are classified as neutral. Bold entries in the table are percentages which are substantial. More detailed tables which cover individual parameters are available on request.

\begin{tabular}{|c|c|c|c|c|c|c|c|c|c|c|}
\hline \multicolumn{6}{|c|}{ Addition } & \multicolumn{5}{|c|}{ Deletion } \\
\hline & $\mathrm{N}(1 \%)$ & $\mathrm{N}(5 \%)$ & Neutral & $\mathrm{P}(5 \%)$ & $\mathrm{P}(1 \%)$ & $\mathrm{N}(1 \%)$ & $\mathrm{N}(5 \%)$ & Neutral & $\mathrm{P}(5 \%)$ & $\mathrm{P}(1 \%)$ \\
\hline \multicolumn{11}{|c|}{$A$} \\
\hline $\mathrm{N}(1 \%)$ & 89.28 & 1.48 & 3.70 & 0.09 & 0.37 & 80.72 & 3.62 & 4.35 & 0.00 & 0.58 \\
\hline $\mathrm{N}(5 \%)$ & 1.20 & 0.00 & 0.37 & 0.00 & 0.00 & 1.45 & 0.29 & 0.58 & 0.00 & 0.00 \\
\hline Neutral & 2.31 & 0.37 & 0.74 & 0.00 & 0.00 & 4.35 & 0.87 & 2.75 & 0.00 & 0.00 \\
\hline $\mathrm{P}(5 \%)$ & 0.00 & 0.00 & 0.00 & 0.00 & 0.00 & 0.14 & 0.00 & 0.00 & 0.00 & 0.00 \\
\hline $\mathrm{P}(1 \%)$ & 0.09 & 0.00 & 0.00 & 0.00 & 0.00 & 0.29 & 0.00 & 0.00 & 0.00 & 0.00 \\
\hline \multicolumn{11}{|c|}{$B$} \\
\hline $\mathrm{N}(1 \%)$ & 0.28 & 0.00 & 0.00 & 0.00 & 0.92 & 0.00 & 0.00 & 0.00 & 0.00 & 0.43 \\
\hline $\mathrm{N}(5 \%)$ & 0.00 & 0.00 & 0.00 & 0.00 & 0.00 & 0.00 & 0.00 & 0.00 & 0.00 & 0.00 \\
\hline Neutral & 0.00 & 0.00 & 0.28 & 0.37 & 1.94 & 0.00 & 0.00 & 1.45 & 0.58 & 2.46 \\
\hline $\mathrm{P}(5 \%)$ & 0.09 & 0.00 & 0.00 & 0.00 & 1.20 & 0.14 & 0.14 & 0.58 & 0.29 & 1.59 \\
\hline $\mathrm{P}(1 \%)$ & 0.65 & 0.00 & 1.39 & 1.48 & 91.40 & 1.01 & 0.00 & 3.77 & 1.88 & 85.65 \\
\hline \multicolumn{11}{|c|}{$\Gamma$} \\
\hline $\mathrm{N}(1 \%)$ & 11.74 & 2.31 & 7.67 & 0.37 & 1.48 & 12.03 & 3.33 & 11.16 & 0.29 & 0.72 \\
\hline $\mathrm{N}(5 \%)$ & 1.48 & 1.11 & 4.44 & 0.28 & 0.09 & 2.61 & 0.29 & 4.20 & 0.29 & 0.43 \\
\hline Neutral & 9.15 & 4.07 & 33.09 & 1.57 & 3.97 & 11.88 & 5.51 & 36.96 & 1.74 & 2.32 \\
\hline $\mathrm{P}(5 \%)$ & 0.55 & 0.37 & 3.42 & 0.18 & 0.55 & 0.72 & 0.14 & 1.01 & 0.14 & 0.14 \\
\hline $\mathrm{P}(1 \%)$ & 1.48 & 0.28 & 6.56 & 0.65 & 3.14 & 0.43 & 0.14 & 2.75 & 0.29 & 0.43 \\
\hline \multicolumn{11}{|c|}{$\Delta$} \\
\hline $\mathrm{N}(1 \%)$ & 1.29 & 0.00 & 0.09 & 0.00 & 1.29 & 2.32 & 0.00 & 0.14 & 0.14 & 1.59 \\
\hline $\mathrm{N}(5 \%)$ & 0.00 & 0.00 & 0.09 & 0.00 & 0.09 & 0.00 & 0.00 & 0.00 & 0.00 & 0.14 \\
\hline Neutral & 0.00 & 0.00 & 0.18 & 0.18 & 1.76 & 0.29 & 0.14 & 1.59 & 0.14 & 4.20 \\
\hline $\mathrm{P}(5 \%)$ & 0.00 & 0.00 & 0.00 & 0.00 & 0.46 & 0.00 & 0.00 & 0.43 & 0.14 & 2.32 \\
\hline $\mathrm{P}(1 \%)$ & 1.02 & 0.09 & 2.50 & 1.20 & 89.74 & 1.88 & 0.14 & 4.78 & 2.61 & 76.96 \\
\hline
\end{tabular}




\section{Table 3.16: Summary of Parameter Dynamics}

The table reports a summary of key cells of parameter dynamics for the sums of the $\alpha \mathrm{s}, \beta \mathrm{s}, \gamma_{\mathrm{s}}$ and $\delta \mathrm{s}$ under OLS, WLS, VAR, $\operatorname{WVAR}, \operatorname{GARCH}(1,1)$ and $\operatorname{BEKK}(1,1)$ for addition and deletion events respectively. The table entries are shown as percentages of stocks with consistency in the sign of model parameters before and after both events. "N" and "P" denotes negative and positive respectively. All other changes at a significance level of which is greater than $5 \%$ are classified as neutral. More detailed tables which cover individual parameters are available on request.

\begin{tabular}{cccccccc}
\hline Parameter & Cell & OLS & WLS & VAR & WVAR & GARCH & BEKK \\
\hline \multicolumn{7}{c}{ Addition } \\
$A$ & $\mathrm{~N}(1 \%)-\mathrm{N}(1 \%)$ & 83.85 & 59.54 & 91.13 & 71.78 & 92.98 & 89.28 \\
$B$ & $\mathrm{P}(1 \%)-\mathrm{P}(1 \%)$ & 92.10 & 81.63 & 91.57 & 81.46 & 92.61 & 91.40 \\
$\Gamma$ & Neutral - Neutral & 45.87 & 44.54 & 48.09 & 37.53 & 44.64 & 33.09 \\
$\Delta$ & $\mathrm{P}(1 \%)-\mathrm{P}(1 \%)$ & 87.49 & 77.99 & 93.17 & 87.58 & 91.59 & 89.74 \\
\multicolumn{7}{c}{ Deletion } \\
$A$ & $\mathrm{~N}(1 \%)-\mathrm{N}(1 \%)$ & 70.88 & 37.23 & 83.52 & 53.02 & 85.80 & 80.72 \\
$B$ & $\mathrm{P}(1 \%)-\mathrm{P}(1 \%)$ & 81.04 & 60.85 & 82.28 & 62.23 & 85.22 & 85.65 \\
$\Gamma$ & Neutral - Neutral & 47.94 & 55.91 & 53.16 & 52.61 & 47.10 & 36.96 \\
$\Delta$ & $\mathrm{P}(1 \%)-\mathrm{P}(1 \%)$ & 72.80 & 56.87 & 81.59 & 75.14 & 78.84 & 76.96 \\
\hline
\end{tabular}


Table 3.17: $Z$ Scores for Tests of Difference in Model Parameters (Addition)

The table is based on 60-day trade data under OLS, WLS, VAR, WVAR, GARCH $(1,1)$ and BEKK $(1,1)$ models for the addition events. Differences are calculated by absolute value of model parameters between after and before the event (After minus Before). $Z$ scores for difference in individual parameters and sums are computed as described in Section 2.3.3. Bold format denotes significance at least at the $1 \%$ level.

\begin{tabular}{ccccccc}
\hline \multicolumn{7}{c}{ Addition } \\
& OLS & WLS & VAR & WVAR & GARCH & BEKK \\
\hline$\alpha_{0}$ & -0.113 & 2.202 & 1.014 & -0.609 & 0.172 & 1.090 \\
$\alpha_{1}$ & -0.678 & 1.246 & -0.006 & 0.001 & -0.339 & -0.889 \\
$\alpha_{2}$ & -2.404 & -1.220 & -0.008 & 0.000 & -1.438 & $\mathbf{- 2 . 9 6 5}$ \\
$\alpha_{3}$ & -1.311 & $\mathbf{- 3 . 3 4 8}$ & -0.020 & -0.006 & 0.002 & -1.661 \\
$\alpha_{4}$ & 0.147 & -0.306 & -0.015 & -0.003 & 0.234 & $\mathbf{- 3 . 7 3 5}$ \\
$\alpha_{5}$ & 1.249 & 2.060 & 0.004 & -0.001 & $\mathbf{4 . 6 3 1}$ & $\mathbf{- 3 . 4 9 6}$ \\
$\beta_{0}$ & 2.488 & $\mathbf{3 . 2 1 9}$ & 1.947 & 2.492 & -0.194 & 1.654 \\
$\beta_{1}$ & $\mathbf{- 3 . 2 8 1}$ & 0.844 & 0.334 & -0.547 & -1.815 & -0.634 \\
$\beta_{2}$ & 0.695 & -2.560 & $\mathbf{2 . 7 4 3}$ & 1.578 & -0.063 & 2.572 \\
$\beta_{3}$ & 0.813 & 1.263 & 1.805 & 1.364 & 0.272 & $\mathbf{2 . 7 4 8}$ \\
$\beta_{4}$ & -0.192 & 1.750 & -0.199 & -0.410 & -0.246 & -0.089 \\
$\beta_{5}$ & -1.720 & 0.556 & 0.107 & 1.035 & -1.002 & -0.327 \\
$\gamma_{0}$ & -1.241 & -0.510 & -1.392 & -0.413 & 0.373 & -0.104 \\
$\gamma_{1}$ & $\mathbf{6 . 8 5 4}$ & $\mathbf{- 5 . 9 5 1}$ & $\mathbf{8 . 2 7 3}$ & $\mathbf{- 1 0 . 4 5 7}$ & $\mathbf{9 . 8 9 6}$ & $\mathbf{7 . 7 9 9}$ \\
$\gamma_{2}$ & $\mathbf{3 . 2 1 2}$ & $\mathbf{- 3 . 4 2 2}$ & $\mathbf{3 . 5 4 9}$ & $\mathbf{- 4 . 5 8 2}$ & $\mathbf{6 . 5 5 4}$ & $\mathbf{5 . 3 4 7}$ \\
$\gamma_{3}$ & $\mathbf{3 . 7 9 3}$ & $\mathbf{- 3 . 4 8 0}$ & $\mathbf{4 . 1 3 6}$ & $\mathbf{- 6 . 8 5 9}$ & $\mathbf{6 . 5 1 7}$ & $\mathbf{3 . 0 8 6}$ \\
$\gamma_{4}$ & $\mathbf{2 . 6 6 5}$ & -2.313 & 2.469 & $\mathbf{- 3 . 5 5 0}$ & $\mathbf{5 . 1 5 4}$ & $\mathbf{2 . 9 0 0}$ \\
$\gamma_{5}$ & -1.021 & $\mathbf{- 2 . 7 0 2}$ & -1.619 & $\mathbf{- 4 . 6 8 5}$ & 1.566 & $\mathbf{4 . 2 0 8}$ \\
$\delta_{1}$ & -0.766 & -2.409 & -0.138 & $\mathbf{- 5 . 1 6 3}$ & -1.348 & $\mathbf{- 4 . 0 0 6}$ \\
$\delta_{2}$ & $\mathbf{- 3 . 5 4 1}$ & -2.058 & $\mathbf{- 3 . 2 9 2}$ & $\mathbf{- 4 . 9 3 7}$ & -1.653 & $\mathbf{- 6 . 0 0 7}$ \\
$\delta_{3}$ & -2.535 & -1.030 & $\mathbf{- 4 . 8 7 2}$ & $\mathbf{- 3 . 0 1 0}$ & $\mathbf{- 3 . 4 4 3}$ & $\mathbf{- 5 . 1 6 7}$ \\
$\delta_{4}$ & 0.605 & -1.640 & -0.752 & $\mathbf{- 2 . 9 1 9}$ & 0.105 & -0.664 \\
$\delta_{5}$ & -0.600 & -0.940 & -1.291 & -1.638 & -1.338 & -1.903 \\
$A$ & -1.195 & -0.620 & -2.177 & $\mathbf{- 3 . 2 1 1}$ & 0.491 & $\mathbf{- 4 . 5 5 2}$ \\
$B$ & 1.386 & $\mathbf{3 . 4 8 7}$ & 2.132 & $\mathbf{2 . 7 5 3}$ & -0.229 & 1.488 \\
$\Gamma$ & $\mathbf{7 . 0 6 8}$ & $\mathbf{- 7 . 3 5 5}$ & $\mathbf{7 . 7 8 8}$ & $\mathbf{- 1 2 . 2 5 7}$ & $\mathbf{1 0 . 9 9 9}$ & $\mathbf{8 . 9 2 8}$ \\
$\Delta$ & $\mathbf{- 3 . 4 8 4}$ & $\mathbf{- 4 . 4 8 9}$ & $\mathbf{- 5 . 7 7 2}$ & $\mathbf{- 9 . 8 0 4}$ & $\mathbf{- 4 . 7 0 1}$ & $\mathbf{- 9 . 6 2 5}$ \\
\hline & & & & & &
\end{tabular}


Table 3.18: $Z$ Scores for Tests of Difference in Model Parameters (Deletion)

The table is based on 60-day trade data under OLS, WLS, VAR, WVAR, GARCH $(1,1)$ and BEKK $(1,1)$ models for the deletion events. Differences are calculated by absolute value of model parameters between after and before the event (After minus Before). $Z$ scores for difference in individual parameters and sums are computed as described in Section 2.3.3. Bold format denotes significance at least at the $1 \%$ level.

\begin{tabular}{lcccccc}
\hline \multicolumn{7}{c}{ Deletion } \\
& OLS & WLS & VAR & WVAR & GARCH & BEKK \\
\hline$\alpha_{0}$ & 1.325 & -0.756 & -1.025 & -0.297 & 1.014 & 0.930 \\
$\alpha_{1}$ & $\mathbf{4 . 8 6 6}$ & 0.839 & 0.005 & 0.000 & 0.731 & $\mathbf{3 . 3 2 5}$ \\
$\alpha_{2}$ & 2.374 & 0.174 & 0.004 & 0.000 & 0.455 & 0.580 \\
$\alpha_{3}$ & 2.088 & 1.132 & 0.000 & 0.000 & 0.559 & 0.821 \\
$\alpha_{4}$ & $\mathbf{3 . 8 1 0}$ & 0.205 & 0.005 & 0.000 & 0.259 & 2.223 \\
$\alpha_{5}$ & $\mathbf{2 . 6 1 6}$ & -0.906 & 0.002 & 0.000 & 1.047 & 0.478 \\
$\beta_{0}$ & 1.335 & 2.380 & 0.460 & 1.787 & 1.976 & 0.423 \\
$\beta_{1}$ & 0.660 & 1.882 & 2.395 & 1.715 & -0.365 & 2.427 \\
$\beta_{2}$ & 1.531 & -0.595 & 0.691 & 0.254 & 0.012 & 0.519 \\
$\beta_{3}$ & 0.668 & 0.852 & 0.039 & -0.390 & 2.288 & 0.055 \\
$\beta_{4}$ & 1.277 & 1.009 & 0.261 & 0.025 & 1.425 & 0.851 \\
$\beta_{5}$ & 0.363 & 1.686 & 0.234 & 0.197 & 0.159 & 0.221 \\
$\gamma_{0}$ & -2.276 & $\mathbf{2 . 6 8 1}$ & -1.739 & $\mathbf{2 . 8 6 7}$ & $\mathbf{- 2 . 7 1 1}$ & -0.079 \\
$\gamma_{1}$ & $\mathbf{5 . 3 9 5}$ & $\mathbf{- 3 . 1 7 4}$ & $\mathbf{5 . 4 7 0}$ & $\mathbf{- 5 . 1 7 7}$ & $\mathbf{6 . 2 2 7}$ & 2.075 \\
$\gamma_{2}$ & $\mathbf{4 . 2 5 1}$ & -2.488 & $\mathbf{4 . 0 9 3}$ & $\mathbf{- 3 . 3 1 6}$ & 2.449 & $\mathbf{3 . 2 0 0}$ \\
$\gamma_{3}$ & $\mathbf{4 . 3 0 2}$ & -0.934 & $\mathbf{3 . 7 1 9}$ & -1.233 & 1.846 & $\mathbf{1 0 . 1 6 5}$ \\
$\gamma_{4}$ & $\mathbf{2 . 5 8 4}$ & -1.520 & 2.319 & -1.603 & 1.951 & 0.713 \\
$\gamma_{5}$ & 1.552 & 0.275 & 1.299 & -0.134 & $\mathbf{3 . 0 2 8}$ & 1.260 \\
$\delta_{1}$ & 0.931 & -0.045 & 0.380 & -0.176 & 1.406 & -0.548 \\
$\delta_{2}$ & 0.367 & 1.275 & -0.081 & $\mathbf{2 . 9 7 6}$ & 0.917 & -0.499 \\
$\delta_{3}$ & 1.780 & -0.461 & $\mathbf{2 . 7 0 2}$ & 0.039 & 0.898 & 1.433 \\
$\delta_{4}$ & -1.267 & -0.302 & -1.039 & 0.438 & 0.834 & -0.995 \\
$\delta_{5}$ & -0.536 & -1.509 & 1.084 & -1.202 & -0.068 & 1.271 \\
$A$ & $\mathbf{4 . 9 2 3}$ & 0.617 & $\mathbf{5 . 3 9 4}$ & $\mathbf{3 . 3 4 7}$ & 1.483 & $\mathbf{2 . 7 8 0}$ \\
$B$ & 1.001 & 2.355 & 1.105 & 1.471 & 0.857 & 0.565 \\
$\Gamma$ & $\mathbf{6 . 1 8 8}$ & $\mathbf{- 3 . 3 7 4}$ & $\mathbf{5 . 8 0 6}$ & $\mathbf{- 4 . 3 7 7}$ & $\mathbf{5 . 1 1 1}$ & $\mathbf{6 . 2 1 8}$ \\
$\Delta$ & 0.754 & -0.354 & 2.565 & 1.032 & 2.259 & 0.211 \\
\hline & & & & & &
\end{tabular}


Appendix E

Other Tables 


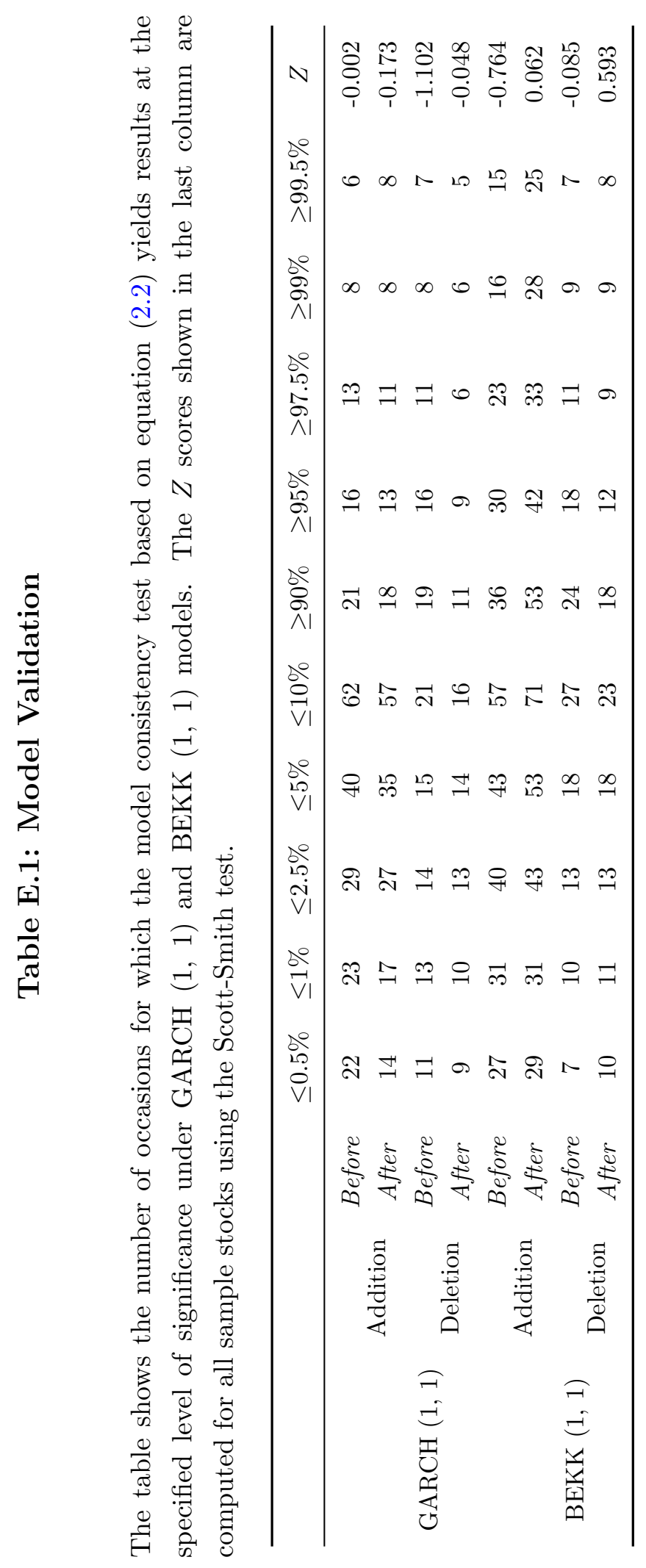




\section{Table E.2: P-Values for Tests of Model Parameters (GARCH)}

Based on the model defined at (3.1) using 60 days of trade data by GARCH $(1,1)$. The tests for individual parameters and sums are computed as described in Section 2.3.3. The cell entries show the estimated parameters and probabilities (p-val.), respectively. Differences are calculated by absolute value of model parameters between after and before both events. Bold format denotes significance at the $1 \%$ level.

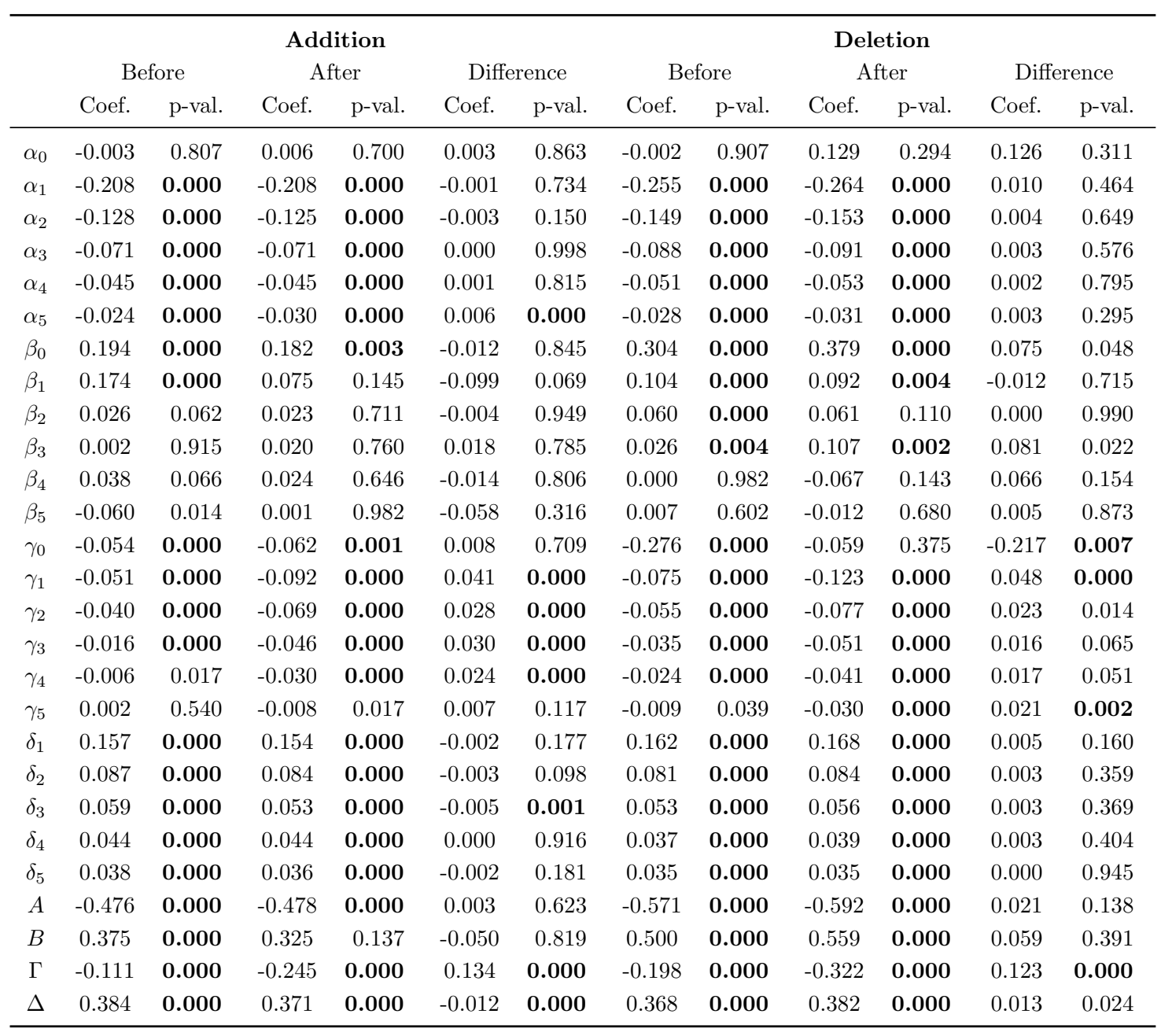




\section{Table E.3: P-Values for Tests of Model Parameters (BEKK)}

Based on the model defined at (3.2) using 60 days of trade data by $\operatorname{BEKK}(1,1)$. The tests for individual parameters and sums are computed as described in Section 2.3.3. The cell entries show the estimated parameters and probabilities (p-val.), respectively. Differences are calculated by absolute value of model parameters between after and before both events. Bold format denotes significance at the $1 \%$ level.

\begin{tabular}{|c|c|c|c|c|c|c|c|c|c|c|c|c|}
\hline & \multicolumn{6}{|c|}{ Addition } & \multicolumn{6}{|c|}{ Deletion } \\
\hline & \multicolumn{2}{|c|}{ Before } & \multicolumn{2}{|c|}{ After } & \multicolumn{2}{|c|}{ Difference } & \multicolumn{2}{|c|}{ Before } & \multicolumn{2}{|c|}{ After } & \multicolumn{2}{|c|}{ Difference } \\
\hline & Coef. & p-val. & Coef. & p-val. & Coef. & p-val. & Coef. & p-val. & Coef. & p-val. & Coef. & p-val. \\
\hline$\alpha_{0}$ & -0.010 & 0.059 & -0.019 & 0.002 & 0.009 & 0.276 & -0.003 & 0.907 & -0.036 & 0.210 & 0.033 & 0.352 \\
\hline$\alpha_{1}$ & -0.193 & 0.000 & -0.192 & 0.000 & -0.001 & 0.374 & -0.228 & 0.000 & -0.235 & 0.000 & 0.007 & 0.001 \\
\hline$\alpha_{2}$ & -0.114 & 0.000 & -0.111 & 0.000 & -0.003 & 0.003 & -0.128 & 0.000 & -0.129 & 0.000 & 0.001 & 0.562 \\
\hline$\alpha_{3}$ & -0.066 & 0.000 & -0.065 & 0.000 & -0.002 & 0.097 & -0.072 & 0.000 & -0.074 & 0.000 & 0.001 & 0.412 \\
\hline$\alpha_{4}$ & -0.041 & 0.000 & -0.038 & 0.000 & -0.004 & 0.000 & -0.041 & 0.000 & -0.045 & 0.000 & 0.004 & 0.026 \\
\hline$\alpha_{5}$ & -0.025 & 0.000 & -0.022 & 0.000 & -0.003 & 0.000 & -0.025 & 0.000 & -0.026 & 0.000 & 0.001 & 0.632 \\
\hline$\beta_{0}$ & 0.203 & 0.000 & 0.225 & 0.000 & 0.022 & 0.098 & 0.384 & 0.000 & 0.423 & 0.000 & 0.039 & 0.672 \\
\hline$\beta_{1}$ & 0.084 & 0.000 & 0.078 & 0.000 & -0.006 & 0.526 & 0.101 & 0.000 & 0.226 & 0.000 & 0.126 & 0.015 \\
\hline$\beta_{2}$ & 0.038 & 0.000 & 0.059 & 0.000 & 0.021 & 0.010 & 0.057 & 0.000 & 0.079 & 0.059 & 0.022 & 0.604 \\
\hline$\beta_{3}$ & 0.005 & 0.448 & 0.034 & 0.000 & 0.029 & 0.006 & 0.014 & 0.480 & -0.017 & 0.685 & 0.003 & 0.956 \\
\hline$\beta_{4}$ & 0.006 & 0.253 & 0.005 & 0.577 & -0.001 & 0.928 & 0.011 & 0.259 & -0.047 & 0.249 & 0.035 & 0.394 \\
\hline$\beta_{5}$ & 0.006 & 0.343 & 0.002 & 0.845 & -0.004 & 0.744 & -0.008 & 0.317 & 0.016 & 0.668 & 0.009 & 0.824 \\
\hline$\gamma_{0}$ & 0.006 & 0.603 & -0.004 & 0.796 & -0.002 & 0.916 & -0.144 & 0.000 & -0.139 & 0.000 & -0.004 & 0.937 \\
\hline$\gamma_{1}$ & -0.056 & 0.000 & -0.087 & 0.000 & 0.030 & 0.000 & -0.074 & 0.000 & -0.084 & 0.000 & 0.010 & 0.038 \\
\hline$\gamma_{2}$ & -0.060 & 0.000 & -0.082 & 0.000 & 0.022 & 0.000 & -0.055 & 0.000 & -0.071 & 0.000 & 0.016 & 0.001 \\
\hline$\gamma_{3}$ & -0.040 & 0.000 & -0.054 & 0.000 & 0.014 & 0.002 & -0.027 & 0.000 & -0.076 & 0.000 & 0.049 & 0.000 \\
\hline$\gamma_{4}$ & -0.019 & 0.000 & -0.030 & 0.000 & 0.011 & 0.004 & -0.015 & 0.000 & -0.019 & 0.000 & 0.003 & 0.475 \\
\hline$\gamma_{5}$ & -0.006 & 0.016 & -0.021 & 0.000 & 0.015 & 0.000 & -0.006 & 0.032 & -0.012 & 0.001 & 0.006 & 0.208 \\
\hline$\delta_{1}$ & 0.155 & 0.000 & 0.150 & 0.000 & -0.005 & 0.000 & 0.157 & 0.000 & 0.156 & 0.000 & -0.001 & 0.583 \\
\hline$\delta_{2}$ & 0.085 & 0.000 & 0.078 & 0.000 & -0.006 & 0.000 & 0.080 & 0.000 & 0.079 & 0.000 & -0.001 & 0.617 \\
\hline$\delta_{3}$ & 0.058 & 0.000 & 0.053 & 0.000 & -0.005 & 0.000 & 0.049 & 0.000 & 0.052 & 0.000 & 0.002 & 0.152 \\
\hline$\delta_{4}$ & 0.041 & 0.000 & 0.040 & 0.000 & -0.001 & 0.507 & 0.035 & 0.000 & 0.034 & 0.000 & -0.002 & 0.319 \\
\hline$\delta_{5}$ & 0.035 & 0.000 & 0.033 & 0.000 & -0.002 & 0.057 & 0.028 & 0.000 & 0.030 & 0.000 & 0.002 & 0.204 \\
\hline$A$ & -0.440 & 0.000 & -0.428 & 0.000 & -0.013 & 0.000 & -0.494 & 0.000 & -0.508 & 0.000 & 0.014 & 0.005 \\
\hline$B$ & 0.341 & 0.000 & 0.403 & 0.000 & 0.062 & 0.137 & 0.559 & 0.000 & 0.682 & 0.002 & 0.123 & 0.572 \\
\hline$\Gamma$ & -0.182 & 0.000 & -0.273 & 0.000 & 0.091 & 0.000 & -0.177 & 0.000 & -0.262 & 0.000 & 0.084 & 0.000 \\
\hline$\Delta$ & 0.374 & 0.000 & 0.355 & 0.000 & -0.018 & 0.000 & 0.350 & 0.000 & 0.350 & 0.000 & 0.001 & 0.833 \\
\hline
\end{tabular}




\section{Chapter 4}

\section{The Effect of Market Returns, Crises, Market Capitalisation, Trading Volume and Short Sales.}

\subsection{Introduction}

The impact of short sales on the speed of price adjustment is crucial to price efficiency in the capital market, given that an efficient price discovery process is defined as the one when stock prices respond immediately to the arrival of new information (Fama, 1991). Diamond \& Verrecchia (1987) predict that short sales constraints impede the speed of price adjustment, especially for negative information by preventing short selling from investors with negative information about fundamentals. This asymmetric speed of price adjustment caused by short sale constraints can become worse in bear than in bull markets. However, regulators believe that short sale constraints or prohibition in bear markets can reduce the speed of price adjustment to bad news which reflects a negative bubble or herding behaviour rather than fundamental information (Beber \& Pagano, 2013). 
Most empirical studies on the speed of price adjustment based on daily or weekly data suggest that short sales improve informational efficiency of prices and that short sales constraints or bans reduce market efficiency and impede the process of price discovery (Beber \& Pagano, 2013; Bris et al., 2007; Saffi \& Sigurdsson, 2010). Based on trade-by-trade high frequency data, researchers including Boehmer \& Wu (2013) and Chen \& Rhee (2010) use the VAR model to characterise more precisely how price efficiency is affected by short sales and document that short selling is an important driver of price discovery.

In line with previous work, Chapter 2 significantly extends the VAR model of Hasbrouck (1991) and investigates the impact of short sales on price efficiency based on higher-frequency data over a 10-year period in the Hong Kong stock market. The Hong Kong market provides a rare opportunity for investigation on the impact of short sales as it only allows stocks which meet certain requirements to be available for short selling and the D-list for short sales is revised on a quarterly basis. In Chapter 2, the results suggest that a number of the stocks in the data set experience significant decreases in the autocorrelations in quote returns and trades implying that price efficiency is improved by short sales, which is consistent with findings from Boehmer \& Wu (2013). These results are robust under the OLS, WLS, VAR and WVAR models. To address the issue of heteroscedasticity caused by high frequency data, Chapter 3 investigates further the impact of short sales on price discovery with consideration of dynamic volatility and co-volatility with application of GARCH and BEKK models. The results of Chapter 3 provide similar evidence supporting that short selling is important to the process of price discovery by capturing the characteristics of heteroscedasticity in the high frequency time series.

This chapter extends the empirical analyses by investigating how the speed of price adjustment is affected by market conditions, market capitalisation, trading volume and short sellers activities. This chapter contributes to the literature in several ways. First, it introduces an extension to the VAR model including a dummy variable of current or one-day lagged market return to explore the impact of market returns on the speed of price adjustment. Moreover, it also includes interaction 
variables in the VAR model using the GARCH framework to deal with the issue of heterogeneity of variance. Secondly, the estimations are conducted in crisis and non-crisis periods in order to examine how speed of price adjustment reacts to the extreme market condition. Thirdly, analyses are conducted based on transaction days with positive market returns and those with non-positive market returns. This method provides evidence on the difference in speed of price adjustment under different market conditions. Fourthly, this chapter provides a direct comparison of the effect of short sales on price efficiency by dividing the sample stocks into four quartiles based on firm size, trading volume and measures of short sales.

The findings of this chapter are summarised as follows. First, the three different approaches to market conditions show that short sales improve the process of price discovery in both good and bad market conditions. Stocks react more quickly to new information in bad markets. By comparing the speed of price adjustment during the recent global financial crisis with non-crisis period, the results show that short sales accelerate informational efficiency in the non-crisis period. However mixed changes in parameters during the crisis period indicate that the effect of short sales is unclear in extreme market conditions.

Secondly, large-sized firms are found to adjust more quickly to new information than small-sized firms and the results are robust under all models. It is also noted that medium-sized addition stocks and small-sized deletion stocks are the most affected by the changing eligibility for short sales. Thirdly, the improvement in price efficiency is more significant when stocks have greater trading volume as they adjust their prices to new information more quickly. Fourthly, the speed of price adjustment to new information is faster for stocks with more shorting activity measured by both short interest and shorting flows. It supports the prediction that short sellers are important participants in the process of price discovery.

The remainder of the chapter is organised as follows. Section 4.2 reviews the relevant literature. Section 4.3 presents the model with interaction variables to capture market conditions. Section 4.4 provides some data description. Section 4.5 reports 
the empirical findings and Section 4.6 concludes.

\subsection{Literature Review}

The determinants of the speed of price adjustment have been explored in the literature and are related to variables including market conditions, firm size, trading volume and shorting activities. This section considers the effects predicted by theory and presents a brief account for the evidences so far.

To measure the loss in price efficiency caused by short sales constraints under different market conditions, Bris et al. (2007) compare among international markets with different institutional settings on short sales constraints. Based on two price efficiency measurements, the downside-minus-upside market $R^{2}$ based on the separate market model estimation conditional on negative and positive market returns and the relative co-movement of an individual stock return with the signed market return lagged one week, they find that prices adjust more quickly to new information especially to bad news in countries where short sales are allowed and practised. Following Bris et al. (2007), Beber \& Pagano (2013) examine the effect of short sales bans on price efficiency around the world during the 2007-09 crisis. They estimate the autocorrelation between stock returns and market returns and compare them between countries with short sales bans and those without bans during the crisis. A significant higher autocorrelation is observed when stocks are subject to a short selling ban and this finding is consistent with a lower speed of price adjustment with shorting bans during the crisis period, especially for negative market returns. Chang et al. (2014) regress weekly stock returns on contemporaneous market returns, conditional on negative and positive market returns. They compare the $R^{2}$ of the market model between down and up markets during the implementation of the pilot scheme for short sales in China in 2010-11. They find that there is a significant lower $R^{2}$ in both down and up markets with short sales activity. It provides supporting evidences that short sales enhance price efficiency not only in the bad market but also in the good market. By adopting the VAR 
model (Hasbrouck, 1991) based on high frequency transaction data, Chen \& Rhee (2010) directly measure the speed of price adjustment in both up and down market conditions and compare the speed difference of stocks before and after the effective date of short selling in the Hong Kong stock market. They divide the daily regression results in the study period into up and down market categories based on the sign of market returns. The trading day is defined as an up (down) market day if it has a positive (non-positive) daily open-to-close market return. The results show that there are significant differences in the speed of price adjustment between shortable and non-shortable stocks in both market conditions while the short sales effect is more significant in a bear market.

Since Lo \& MacKinlay (1990) find the lead-lag cross-autocorrelations in the returns of larger-sized firms generally leading those of smaller ones, the sources of this lead-lag cross-autocorrelations as a delayed speed of new information has been debated by academics. Lo \& MacKinlay (1990) find that the correlation between current small firm's returns and lagged large firm's returns is higher than that between current large firm's returns and lagged small firm's returns. They believe that the lead-lag cross-autocorrelations is caused by the stronger price stickiness or the tendency to adjust more slowly to new information for small firms. Lo \& MacKinlay (1990) believe that firm size is a significant determinant of the crossautocorrelation patterns in stock returns.

By controlling for firm's size effect, Chordia \& Swaminathan (2000) examine the cross-autocorrelation patterns between high volume and low volume stocks. They use the ratio of the number of shares traded in a day to the number of shares outstanding to measure of the trading volume. This specific definition of trading volume can offset the size effect on the raw trading volume as they find a high correlation between firm size and raw trading volume. By conducting the VAR model involving pairs of high and low volume portfolios, they find that daily and weekly returns with high volume lead those with low volume even in the largest size quartile, indicating that trading volume plays an independent role in explaining the cross-autocorrelation patterns and is not driven by firm size. Chordia \& Swaminathan (2000) suggest that infrequently traded stocks respond more slowly 
to information in market returns than actively traded stocks.

Nevertheless, the impact of firm size and trading volume on the speed of price adjustment found in recent empirical studies is controversial. Consistent with Lo \& MacKinlay (1990), Saffi \& Sigurdsson (2010) find that firms with larger market capitalisation are associated with less price delay based on a research of 26 countries. However, Saffi \& Sigurdsson (2010) do not find a significant impact of turnover on price efficiency. Chen \& Rhee (2010) report that the speed of price adjustment for smaller firms is even faster than the larger firms in the Hong Kong stock market. Their finding on trading volume is consistent with Chordia \& Swaminathan (2000) that stocks traded more frequently react more quickly than those traded less frequently.

In theoretical models of Diamond \& Verrecchia (1987), short sellers are assumed to be rational informed traders. They point out that short sellers improve price efficiency by pulling overpriced stocks back to their fundamental levels. They suggest that informed traders are less likely to short for liquidity reasons as short sales are costly, and therefore high short interest from informed traders conveys adverse (negative) information about the stock fundamentals. Most empirical studies show that short sellers are well informed. By using monthly short interest data, Desai et al. (2002) reveal that short sellers have private information and their shorting targets are the overpriced firms. Their findings show that heavily shorted firms experience significantly negative abnormal returns and the negative abnormal returns are increasing in the level of short interest. By using shorting flow data, Boehmer et al. (2008) find that stocks, which are heavily shorted by institutions, underperform significantly compared to stocks that are lightly shorted by institutions. Following Boehmer et al. (2008), Boehmer \& Wu (2013) investigate the impact of short sellers' daily activities on price discovery. They believe that short sellers are important economic drivers of price discovery as they are informed traders with private information. The more active short sellers are, the closer stock prices are to their fundamental levels and the faster stock prices incorporate the public information. Using the daily shorting flow standardised by the stock's daily share trading volume, Boehmer \& Wu (2013) find that more shorting flow only not makes 
stock prices more accurate but also accelerates the speed of price adjustment to the arrival of new information by reducing price delays.

\subsection{Model for the Effect of Market Conditions}

One of the aims of this chapter is to examine the effect of market returns on the process of price discovery when stocks become shortable/non-shortable. Chen \& Rhee (2010) compare the speed of price adjustment between up and down markets based on their analysis of intraday data on a single day using the VAR model. As Chen \& Rhee (2010) estimate the VAR model on a daily basis, it is straightforward to divide the estimation results into two groups for comparison: those with positive daily open-to-close market returns and those with daily open-to-close negative market returns. As described in Chapter 2, a close examination of the data used in the study reveals that during the 60-day period before and after addition and deletion events over a 10 -year period, there are $17.9 \%$ and $39.0 \%$ of the total transaction days with less than 20 trades respectively. Estimating a small number of transactions on a daily basis could cause multi-collinearity and other statistical problems. The model estimations in this study therefore are based on the 60 days before and after addition and deletion events as a whole period. To explore this issue, interaction variables with the dummy variable of market conditions and the corresponding parameters are introduced in the model as follows

$$
\begin{aligned}
& R_{t}=\alpha_{0}+\sum_{i=1}^{5} \alpha_{i} R_{t-i}+\sum_{i=0}^{5} \beta_{i} X_{t-i}+\beta_{m, R} D_{m, t}+\sum_{i=1}^{5} \alpha_{i, D}\left(R_{t-i} \times D_{m, t}\right)+\sum_{i=0}^{5} \beta_{i, D}\left(X_{t-i} \times D_{m, t}\right)+\varepsilon_{R t}, \\
& X_{t}=\gamma_{0}+\sum_{i=1}^{5} \gamma_{i} R_{t-i}+\sum_{i=1}^{5} \delta_{i} X_{t-i}+\beta_{m, X} D_{m, t}+\sum_{i=1}^{5} \gamma_{i, D}\left(R_{t-i} \times D_{m, t}\right)+\sum_{i=1}^{5} \delta_{i, D}\left(X_{t-i} \times D_{m, t}\right)+\varepsilon_{X t},
\end{aligned}
$$

where $D_{m, t}$ is a dummy variable denoted as 1 if the market return on that day is positive or 0 if it is non-positive. The rest of the variables in the model at (4.1) above keep the same definition as in Chapters $2 \& 3$. 
Interpretations of the coefficients on interaction variables are as follows. The positive (negative) $\sum_{i=1}^{5} \alpha_{i, D}$ indicates that the magnitude of quote autocorrelations becomes smaller (greater) with positive (negative) market returns as the quote autocorrelations are initially negative. The positive (negative) $\sum_{i=0}^{5} \beta_{i, D}$ indicates that price impact of trades is enhanced (reduced) with positive (negative) market returns as the price impact of trades are initially positive. The impact of market returns on Granger causality depends on the sign of $\sum_{i=1}^{5} \gamma_{i, D}$ as well as the initial sign of Granger causality. The positive (negative) $\sum_{i=1}^{5} \delta_{i, D}$ indicates that trade continuity is increased (decreased) with positive (negative) market returns as the trade continuity is initially positive. As for the measurements of speed of price adjustment, the negative (positive) $\sum_{i=1}^{5} \alpha_{i, D}$ and the positive (negative) $\sum_{i=1}^{5} \delta_{i, D}$ indicate that the speed of price adjustment is slower when market condition is good (bad).

The dummy variable of market returns used in the model at (4.1), however, embodies foresight. That is, a quote-based return measured at an arbitrary time of the day would be associated with the dummy variable computing using the price at the end of the day. This means that the model can in principle offer an explanation of price discovery under different market conditions, but cannot be used for forecasting purpose. To overcome the foresight issue, an extension of the model is to use market return on the previous day. These results are also included in this chapter.

The VAR model with interaction variables defined at (4.1) is estimated by OLS and WLS to compare the speed difference in price adjustment under different market conditions before and after the event. Furthermore, this chapter also employs the GARCH models of Chapter 3, which are defined as follows 


$$
\begin{gathered}
R_{t}=\alpha_{0}+\sum_{i=1}^{5} \alpha_{i} R_{t-i}+\sum_{i=0}^{5} \beta_{i} X_{t-i}+\beta_{m, R} D_{m, t}+\sum_{i=1}^{5} \alpha_{i, D}\left(R_{t-i} \times D_{m, t}\right)+\sum_{i=0}^{5} \beta_{i, D}\left(X_{t-i} \times D_{m, t}\right)+\varepsilon_{R t}, \\
\sigma_{R, t}^{2}=\omega_{R}+\varphi_{R} \varepsilon_{R, t-1}^{2}+\phi_{R} \sigma_{R, t-1}^{2}, \\
X_{t}=\gamma_{0}+\sum_{i=1}^{5} \gamma_{i} R_{t-i}+\sum_{i=1}^{5} \delta_{i} X_{t-i}+\beta_{m, X} D_{m, t}+\sum_{i=1}^{5} \gamma_{i, D}\left(R_{t-i} \times D_{m, t}\right)+\sum_{i=1}^{5} \delta_{i, D}\left(X_{t-i} \times D_{m, t}\right)+\varepsilon_{X t}, \\
\sigma_{X, t}^{2}=\omega_{X}+\varphi_{X} \varepsilon_{X, t-1}^{2}+\phi_{X} \sigma_{X, t-1}^{2} .
\end{gathered}
$$

Diagnostic tests for ARCH effects described in Chapter 3 will be applied based on the OLS and WLS models with interaction variables in this chapter.

The VAR and WVAR models of Chapter 2 and the BEKK model of Chapter 3 are not used in this chapter in conjunction with the model at (4.2). This is because incorporation of the dummy variables would require the development of purpose built software which is beyond the scope of the thesis. Given the general similarity of results from the OLS, WLS and GARCH models, this is not considered to be a major limitation.

\subsection{Data Description}

This chapter utilises the same high frequency quotes and trades dataset of Chapters $2 \& 3$ in a 10-year period from May 2001 and May 2011 sourced from the HKEx.

To examine how speed of price adjustment affected under different market conditions when stocks become shortable or non-shortable, three different approaches are applied in this chapter. First, the models with interaction variables are used to investigate the sensitivity of speed of price adjustment under the current market condition and the one-day lagged market condition. Daily market return is calculated by the Hang Seng daily market index sourced from Datastream. Secondly, the estimation results based on Chapters $2 \& 3$ before and after the event are divided into two categories by crisis and non-crisis periods to provide alternative analysis on how speed of price adjustment reacts to the extreme market condition. By using the recession period from the National Bureau of Economic Research [NBER 
henceforth], the crisis period is defined as December 2007 and June $2009^{14}$ which refers to the recent global financial crisis ${ }^{15}$. The event is defined in the crisis period if the whole estimation period of the event (60 days before and after the effective date) is in the crisis period. Thirdly, the 60-day data before and after each event for each stock is divided into two groups, transactions on the days with positive and non-positive market returns respectively. For each stock, six models including OLS, WLS, VAR, WVAR, GARCH and BEKK are estimated under up and down markets before and after each event. The first quote return and trade variables on each day is treated as a missing value since the time duration between trades on non-continuous days would be large.

To examine the impact of market capitalisation, trading volume, activities of short sellers including short interest and shorting flow on the speed of price adjustment during addition and deletion events, information on daily market capitalisation, daily trading volume and total shares outstanding is obtained from Datastream. Daily short sales data is sourced from the HKEx.

The analysis based on the current and one-day lagged market return in this chapter contains three models including OLS, WLS, and GARCH. The subgroup analysis based on crisis period, up and down markets, size, trading volume and shorting activities in this chapter contains six models including OLS, WLS, VAR, WVAR, GARCH and BEKK. Time-weighted variables provide alternative solution to heteroscedasticity, and therefore GARCH framework is not implemented with the weighted variables to avoid that the variance dynamics is over specified.

\footnotetext{
${ }^{14}$ NBER recession periods are available on the website of http://www.nber.org/cycles.html.

${ }^{15}$ The paper by Boehmer \& Wu (2013) does not specify the crisis period explicitly and so no direct comparison may be made. The models have been re-estimated using Beber \& Pagano (2013) who use the period January 2008 to June 2009 as the crisis period. Not surprisingly the results are the same since the crisis periods differ by one month but the short selling lists are revised quarterly.
} 


\subsection{Results}

This section presents the results on changes in speed of price adjustment during both addition and deletion events by market conditions, market capitalisation, trading volume and short sales activities. The SS test used in this chapter is defined the same as in Section 2.3.3 and the significance level is at 1\%. Section 4.5.1 reports the results by using three approaches dealing with good and bad market conditions. It also reports the diagnostic tests for the $\mathrm{ARCH}$ effects introduced in Section 3.2.3 for univariate models interacted with dummy variable for current and one-day lagged market return (OLS and WLS), respectively. Sections 4.5.2, 4.5.3 and 4.5.4 report analyses for the speed of price adjustment by size, trading volume and shorting activities, respectively.

\subsubsection{Market Conditions}

To investigate the process of price discovery under different market conditions during addition and deletion events, three approaches are used in this section. Section 4.5.1.1 reports the results based on OLS and WLS models interacted with a dummy variable of different market returns to explore the reaction of speed of price adjustment to current and one-day lagged positive and non-positive market returns. Section 4.5.1.2 presents the analysis based on the estimation results from Chapters $2 \& 3$ broken down by crisis and non-crisis periods. Section 4.5.1.3 provides the estimation results based on the transaction days divided into two groups, days with positive market returns and days with non-positive market returns.

\subsubsection{Dummy Variable of Current and One-day Lagged Market Re- turn}

This section first presents diagnostics tests for ARCH effects for the model at (4.2). It then presents results for current and one-day lagged market return, respectively. 


\section{Diagnostic Tests for ARCH effects}

For addition events, Table 4.1 reports the diagnostic tests results based on OLS interacted with a dummy variable of current market condition, showing the percentage of event stocks at the corresponding significance level. For intraday data, the significant levels are $0.1 \%, 1 \%$ and $5 \%$. Any results with a significance level greater than $5 \%$ are classified as neutral. The $J B$ test is used to test for normality of residuals. The Portmanteau $Q$ test ( $Q$ test) and the Lagrange Multiplier Test ( $L M$ test) are used to test the significance of ARCH effects. Numbers in the brackets denotes the number of lags of the residual series. Four vertical sections are for $R$ and $X$ equations in the model before and after the event. Bold format in the table denotes the greatest percentage value.

[Insert Table 4.1 about here]

Table 4.1 shows that the residuals of nearly all of the sample stocks do not follow normality, which represents a white noise process at a significance level of $1 \%$. At least half of the sample stocks experience ARCH effects at a significance level of $1 \%$ as shown by $Q$ test and $L M$ test. Furthermore, there are more stocks with significant $\mathrm{ARCH}$ effects in the $R$ equation before and after the addition events compared with the $X$ equation. The results in Table 4.2 report the similar results for the deletion events. Although the percentage of event stocks with significant ARCH effects reduces in the $X$ equation compared to the addition events, there are still more than approximately $42 \%$ of the sample stocks in the $X$ equation and $79 \%$ in the $R$ equation present existence of the ARCH effect at the significance level of $1 \%$.

[Insert Table 4.2 about here]

Tables $4.3 \& 4.4$ present the corresponding diagnostic test results based on the WLS models. It is noted that there are less stocks experiencing the significant ARCH effects in both $R$ and $X$ equations during both events when variables in 
the models are time-weighted, compared with those under OLS models. There are approximately less than $50 \%(40 \%)$ of event stocks with significant ARCH effect at the level of $1 \%$ for addition (deletion) events when time duration is taken into account.

[Insert Tables 4.3 and 4.4 about here]

Furthermore, Tables 4.5, 4.6, 4.7 and 4.8 report similar results for diagnostic tests for ARCH effects for OLS and WLS models interacted with a dummy variable of one-day lagged market return.

[Insert Tables 4.5, 4.6, 4.7 and 4.8 about here]

Based on the results shown above, GARCH framework is applicable to the OLS model interacted with the dummy variable of current market return and one-day lagged market return.

\section{Dummy Variable for Current Market Return}

Tables 4.9 shows the p-value for tests of model parameters by OLS interacted with a dummy variable of current market return for addition and deletion events using the model defined at (4.1). The tests for individual parameters and sums are computed as described in Section 2.3.3. The cell entries show the estimated parameters and their probabilities, respectively. Differences are calculated by absolute value of model parameters between after and before both events. Bold format in the table denotes significance at the $1 \%$ level.

In Table 4.9, there are significantly positive relationships between the dummy variable of current market return and quote returns as well as trades before and after 
addition and deletion events. It suggests the average transaction trend of the sample stocks and the current market are moving in the same direction. No significant changes in the dummy variables, $D_{m, R}$ and $D_{m, X}$, during both events indicate that the co-movement between individual stocks and the market is not affected by short sales. Parameters measuring price efficiency interacted with the dummy variable of the current market return stay insignificant before and after both events and this implies that there are no significant differences in the speed of price adjustment between good and bad current market conditions.

[Insert Table 4.9 about here]

Tables 4.10 shows the p-value for tests of model parameters for the WLS model interacted with dummy variable of current market return for addition and deletion events. Table 4.10 reports that the relationship with current market return remains significantly positive for trades before and after both events while the relation will disappear for quote returns when stocks become non-shortable. For the sums of parameters, only the aggregated price impact of trades, $\mathrm{B}_{D m}$, are found significantly negative before the deletion events. It suggests that the price impact of trades for shortable stocks is greater when the current market condition is bad. However, it is found that the speed differences of price adjustment are not significant under different current market conditions under the time-weighted model.

[Insert Table 4.10 about here]

Table 4.11 presents the corresponding results for the GARCH model. Similar to those under OLS and WLS, the findings do not provide strong evidence to support that current market conditions affect the aggregated parameters, as most of the estimated parameters of the proxies for price efficiency do not change significantly.

[Insert Table 4.11 about here] 
In sum, the application of interaction variables with a dummy variable of current market return in the models do not provide strong evidence supporting that the speed of price adjustment is significantly impacted by current market conditions.

\section{Dummy Variable for One-day Lagged Market Return}

As discussed in Section 4.3, a dummy variable for current market return used in the models at (4.1) and (4.2) raises foresight issues. Quote returns and trades at an arbitrary time of the day would be associated with the dummy variable computed by the price at the end of the day. To resolve this issue, this section considers the model with interaction variables based on the sign of one-day lagged market return.

Table 4.12 reports the p-value for tests of model parameters by OLS interacted with a dummy variable of one-day lagged market return for both events. $D_{\text {mlag }}$ is a dummy variable set to one if the one-day lagged market return is positive and zero otherwise. It is found that the aggregated parameter of interaction variable with trade autocorrelations $\left(\Delta_{\text {Dmlag }}\right)$ before the addition events is 0.009 , which is significant at the $1 \%$ level. This positively significant parameter $\Delta_{\text {Dmlag }}$ implies that trade continuity is stronger when the one-day lagged market return is positive and it indicates that stocks have quicker reaction to new information when the market on the previous day was bad.

[Insert Table 4.12 about here]

Table 4.13 shows the corresponding results for WLS. The results show that the aggregated parameter $\Delta_{\text {Dmlag }}$ after the addition events is 0.013 significant at the $1 \%$ level. This positive value implies that the stickiness of trades are stronger when the market return on the previous day is good. The findings support that stocks 
have shorter price delay if they are under a bad previous-day market return.

[Insert Table 4.13 about here]

Table 4.14 present the results for the GARCH model. The insignificant aggregated parameters interacted with the dummy variable, $A_{\text {Dmlag }}$ and $\Delta_{\text {Dmlag }}$, show that the effect of one-day lagged market conditions on the speed of price adjustment is not visible during both events when heteroscedasticity is taken into account.

[Insert Table 4.14 about here]

The overall results based on the dummy variable of current and one-day lagged market return suggest that the speed of price adjustment is not impacted by the current market conditions while it is affected by the one-day lagged market conditions mainly with attribution to the changes in trade continuity. Stocks have stronger trade continuity if the market on the previous day is good which reveals that stocks react more quickly to the news under the bad market.

\subsubsection{Crisis Period}

This section carries on an investigation of the speed of price adjustment in the crisis period. The estimation results from Chapters $2 \& 3$ are divided into two categories: crisis and non-crisis periods. According to the records from NBER, the crisis period is defined as the period between December 2007 and June 2009.

Table 4.15 shows the tests for sums $A$ and $\Delta$ measuring speed of price adjustment for addition stocks under all estimation models in crisis and non-crisis periods. The cell entries of the first panel show the number of observations in each period. The cell entries of other panels show the estimated parameters and their probabilities under each model, respectively. Differences are calculated by absolute value 
of model parameters between the corresponding periods. For addition stocks, in the non-crisis period, parameters measuring price efficiency experience a significant decrease implying short sales accelerate the speed of price adjustment. However, results in the crisis period are mixed as trade continuity decreases for all estimation models while autocorrelation in quote returns increases under time-weighted models. Comparing the differences in parameters between crisis and non-crisis periods, the mixed results do not result in a clear picture showing that the speed of price adjustment is significantly different in the crisis period.

[Insert Table 4.15 about here]

Table 4.16 reports the results for deletion stocks. For both periods, the increases in the sums $A$ and $\Delta$ reveal that stocks have longer price delay when they are removed from the D-list. Similar to those for the addition events, the differences in the speed of price adjustment between crisis and non-crisis period for deletion stocks stay inconclusive.

[Insert Table 4.16 about here]

To conclude, short sales recover the process of price discovery in the non-crisis period. However the results are mixed for the crisis period as the efficiency-enhancing effect of short sales is not clear under the time-weighted models. In addition, no clear evidence is found to support that there are significant differences in the speed of price adjustment between the crisis and the non-crisis periods. Table F.1 in Appendix F reports the cumulative abnormal returns based on the market model estimated before, during and after the crisis. The period of crisis is as defined above (December 2007 to June 2009). For the market model, an estimation window of $(-240,-60)$, with a minimum length of 180 days, is used. Each panel of the table indicates that the minimum length of time for stocks to be added back after deletion from the D-list. 
As shown in Table F.1, the daily estimated cumulative abnormal returns are greater than zero at the $1 \%$ level of significance during the crisis period for the event windows of 30 and 60 days. If the stocks had been deleted from the D list following the standard procedures, it is to be expected that the abnormal returns would be zero or negative. The positive cumulative daily abnormal returns in the crisis panel indicate that stocks were not deleted from the D-list during the crisis period because of poor performance. It is therefore considered likely that deletion served as a substitute for a ban on short sales in the Hong Kong stock market. This finding is supported qualitatively by the small number of stocks added to the D-list during the crisis period compared to the number of additions both before and afterwards. The lack of clear effects being associated with the crisis period for addition stocks reported in Table 4.15 may be caused by the small number of stock/events imposed by the market regulator.

\subsubsection{Up and Down Markets}

Based on the method described in Section 4.4, this section provides an alternative examination on the process of price discovery under different market conditions. The 60-day trade-by-trade data before and after each event for each stock is divided into two groups by the sign of the market returns on that day including positive (up) and non-positive (down) returns. For each stock, the six models used in this thesis are estimated based on these two groups, namely up and down markets respectively. Table 4.17 reports that there are significant decreases in the autocorrelations of quote returns and trades indicating that price efficiency is enhanced by short sales in both up and down markets for the addition stocks. Moreover, the differences between up and down markets show that stocks respond more quickly in the down market rather than in the up market. For the deletion events, the similar results are observed in Table 4.18. Price efficiency becomes worse when stocks are removed from the D-list regardless of market conditions. However, no significant differences in the speed of price adjustment are found under the majority of estimation models between the up and the down markets for the deletion stocks. 
[Insert Tables 4.17 and 4.18 about here]

To conclude, a faster process of price discovery is associated with short sales no matter whether market return is up or down. In general, stocks adjust their prices more quickly to new information in down markets rather than in up markets. This finding is consistent with the uncertain information hypothesis of Brown et al. (1988) that stock price reactions to bad news (down markets) are stronger than good news (up markets). With a higher aversion to downside risk, investors react faster to bad news. For instance, institutional investors are quicker to respond to unfavourable information as they feel that they would be penalized more if they underperform in a bear market than in a bull market (Sortino \& Van Der Meer, 1991). Furthermore, the cost of not adjusting prices downward is higher than the cost of not adjusting prices upward as the former involves building up inventory with overpriced securities to maintain price continuity.

\subsubsection{Market Capitalisation}

This section explores the effect of market capitalisation on the speed of price adjustment during both events by dividing the estimation results into four-sized quartiles. For each addition and deletion event, stocks are ranked by their average daily market value calculated in the previous 60 days before the effective date. Group values are assigned to each stock ranging from 0 to 3 for quartiles. For instance, quartiles partition the market values into four groups, with the smallest values receiving, by default, a quartile value of 0 and the largest values receiving a quartile value of 3 . The formula for calculating group values is:

$$
\operatorname{FLOOR}(\operatorname{rank} * k /(n+1))
$$

where FLOOR is the function that returns the largest integer that is less than or equal to the argument, fuzzed to avoid unexpected floating point results; rank 
is the value's order rank; $k$ is the number of groups; $n$ is the number of observations having non-missing values of the ranking variables. MVRANK $=1$ indicates the smallest market size group while MVRANK $=4$ indicates the largest market size group. Sums of $A$ and $\Delta$ are the average values within each size quartile. Table 4.19 presents the results based on the size quartiles for the addition events. Stocks in all size quartiles experience a significant decrease in the autocorrelation of quote returns and trades which indicates that all the stocks regardless their market capitalisations react more quickly when they are allowed for short sales. The differences between the biggest and the smallest firms after the addition events reveal that large firms are faster in incorporation of new information in the market. It is also found that the medium-sized firms are affected most by short sales during the addition events with robustness under all models.

[Insert Table 4.19 about here]

Table 4.20 shows the results of size quartiles for the deletion events. Stocks for all size quartiles have significant increased autocorrelations in quote returns and trades under a majority of models and it implies that there is a greater price delay after stocks being removed from the D-list for short sales. Similar to the results for the addition events, the significant differences between the largest and the smallest show that large firms still respond more quickly when they are removed from the D-list. The results also indicate that small firms are the most affected with robustness under all models during the deletion events.

[Insert Table 4.20 about here]

To sum up, the overall results show that short sales strengthen price efficiency regardless of firm size. The speed of price adjustment of larger firms is quicker than smaller firms for both events. Medium-sized firms are the most influenced during the addition events while small-sized firms are the most affected during the 
deletion events.

\subsubsection{Trading Volume}

This section investigates the effect of trading volume on the speed of price adjustment during both events by dividing estimation results into four volume quartiles. The trading volume is the ratio of the number of shares traded to the total number of shares outstanding. This measure is used as it offsets the size effect on the raw trading volume, as there is a high correlation between size and raw trading volume (Chordia \& Swaminathan, 2000). For each addition and deletion event, stocks are ranked by their average daily trading volume calculated in the previous 60 days before the effective date. VORANK $=1$ indicates the lowest trading volume group while VORANK $=4$ indicates the highest trading volume group. Sums of $A$ and $\Delta$ are the average values within each volume quartile. In Table 4.21 for addition stocks, results under the majority of models show a significant decrease in the autocorrelation of quote returns and trades although there are a few mixed evidences in changes under GARCH. The first and second largest volume quartiles exhibit significant changes under all models and it reveals that stocks traded more frequently are more likely to be affected by short sales than those traded infrequently during the addition events. The comparison between the most and the least traded groups indicates that, in general, stocks with more trading activities spend less time to adjust their prices under the unweighted models.

[Insert Table 4.21 about here]

Table 4.22 shows the results within four volume quartiles for deletion stocks. The sample stocks within all volume quartiles experience a significant increase in the autocorrelations of the corresponding variables indicating that price efficiency gets worse when stocks become non-shortable. Contrary to those in the addition events, stocks from the top and the bottom of the quartile are affected most during the deletion events. Moreover, it also supports that the speed of price adjustment is 
faster when stocks are traded more frequently.

[Insert Table 4.22 about here]

In conclusion, short sales generally accelerate the speed of price adjustment during addition and deletion events. Price efficiency is more likely to be affected for stocks with higher trading volume when they are added to the D-list while stocks with the highest and the lowest trading volume are impacted most during the deletion events. Stocks traded more frequently respond more quickly in price adjustment to new information and this is robust under the models which are not time-weighted.

\subsubsection{Shorting Activity}

This section uses short interest (Desai et al., 2002) and shorting flow (Boehmer et al., 2008) to measure the effect of short sellers' activities on the speed of price adjustment. Note that shorting activity quartiles are formed based on the daily short sales data which is only available after the addition events and before the deletion events. It means that the analysis of shorting activity approaches may face foresight issues. Therefore, although the results can offer an explanation of price discovery they are not suitable to be used for forecasting purposes.

\subsubsection{Short Interest}

The short interest is the ratio of the number of shares sold short to the total number of shares outstanding on a daily basis. For each addition and deletion event, stocks are ranked by their short interest calculated in the 60-day period after the addition events and before the deletion events. SIRANK $=1$ indicates the group for stocks with the lowest short interest and SIRANK $=4$ indicates the group for stocks with the highest short interest. Sums of $A$ and $\Delta$ are the average values 
within each short interest ratio quartile.

In Table 4.23 for addition stocks, the results show that stocks under all models experience a significant improvement when they become shortable with significant decreases in autocorrelations in quote returns and trades after the addition events. The differences between the most shorted stocks and the least shorted stocks reveal that addition stocks are more efficient in price discovery if they are more frequently traded by short sellers under all unweighted models.

[Insert Table 4.23 about here]

Table 4.24 reports the results for deletion stocks based on the short selling interest before the event. In general, stocks lose their price efficiency shown as significant increases in autocorrelations in quote returns and trades except for the case under the BEKK model. The mixed results on the price comparison between two short interest quartiles $4 \& 1$ imply that the correlation between the speed of price adjustment and the level of short interest is unclear for deletion stocks.

[Insert Table 4.24 about here]

\subsubsection{Shorting Flow}

The shorting flow is the ratio of the stock's daily shorting volume to its daily trading volume. This ratio makes shorting activity comparable across stocks with different trading volumes (Boehmer et al., 2008). For each addition and deletion event, stocks are ranked by their average daily shorting flow in the following 60 days after the addition event or in the previous 60 days before the deletion event. SFRANK $=1$ indicates the lowest shorting flow while SFRANK $=4$ indicates the highest shorting flow. Sums of $A$ and $\Delta$ are the average values for each shorting flow quartile. In Table 4.25 for addition stocks, similar to the results from short 
interest, it shows that short sales enhance the process of price discovery for addition stocks for each shorting flow quartile under all models with significant decreases in autocorrelations. Again, significant decreases in the parameter autocorrelations are observed between stocks with the highest shorting flow and those with the lowest shorting flow and the findings are robust with all models. It suggests that more shorting flows are associated with a better price discovery for addition stocks.

[Insert Table 4.25 about here]

Table 4.26 reports the results for the deletion events. Except for the results based on the VAR model, stocks becoming non-shortable have slower speed of price adjustment shown by some significant increases in autocorrelations after the event. Consistent with the results for the addition stocks, stocks with higher shorting flows exhibit better price efficiency.

[Insert Table 4.26 about here]

To sum up, the analysis of short sellers' activities including short interest and shorting flow suggests that short sellers' trading activities contributes significantly to the process of price discovery and in general stock prices respond more quickly if short sellers are more active in the market.

\subsection{Conclusions}

This chapter conducts more detailed empirical analyses by examining how the process of price discovery is impacted under different market conditions, market capitalisation, trading volume and short sellers' activities during both addition and deletion events in the Hong Kong stock market for the 10-year study period. 
For market conditions, three different approaches are applied for investigation including models interacted with the dummy variable of current and one-day lagged market return, subgroup analysis by dividing the study period into crisis and noncrisis periods, re-estimation of the models in Chapter $2 \& 3$ based on the days with positive and non-positive market returns, respectively. Taken together, the results suggest that short sales enhance the speed in incorporation of all available information into prices regardless of the market status. Comparing up and down markets, stock prices adjust more quickly to the information in a bearish market. By looking at the recent global financial crisis, the results show that short sales improve price efficiency during the non-crisis period however the effect of short sales remain unclear during the crisis.

For the size effect, there is a positive relationship between firm size and the corresponding speed of information. Larger-sized firms absorb the news faster than smaller-sized firms. The results are consistent with those from Lo \& MacKinlay (1990) that small firms have stronger price stickiness or the tendency to adjust more slowly to new information. Medium-sized addition stocks and small-sized deletion stocks are the most affected groups during these events under all estimation models. For the volume effect, stocks with higher trading volume are faster on information assimilation under unweighted models. Based on two different measures as the proxies for short sellers' activity, the findings suggest that short sellers play an important role in the information efficiency of prices. Higher short interest is associated with shorter price delays under the models without consideration of time duration. More shorting flows are found to be a significant determinant of faster speed of price adjustment to new information. Both proxies suggest that prices incorporate information faster when short sellers are more active. 


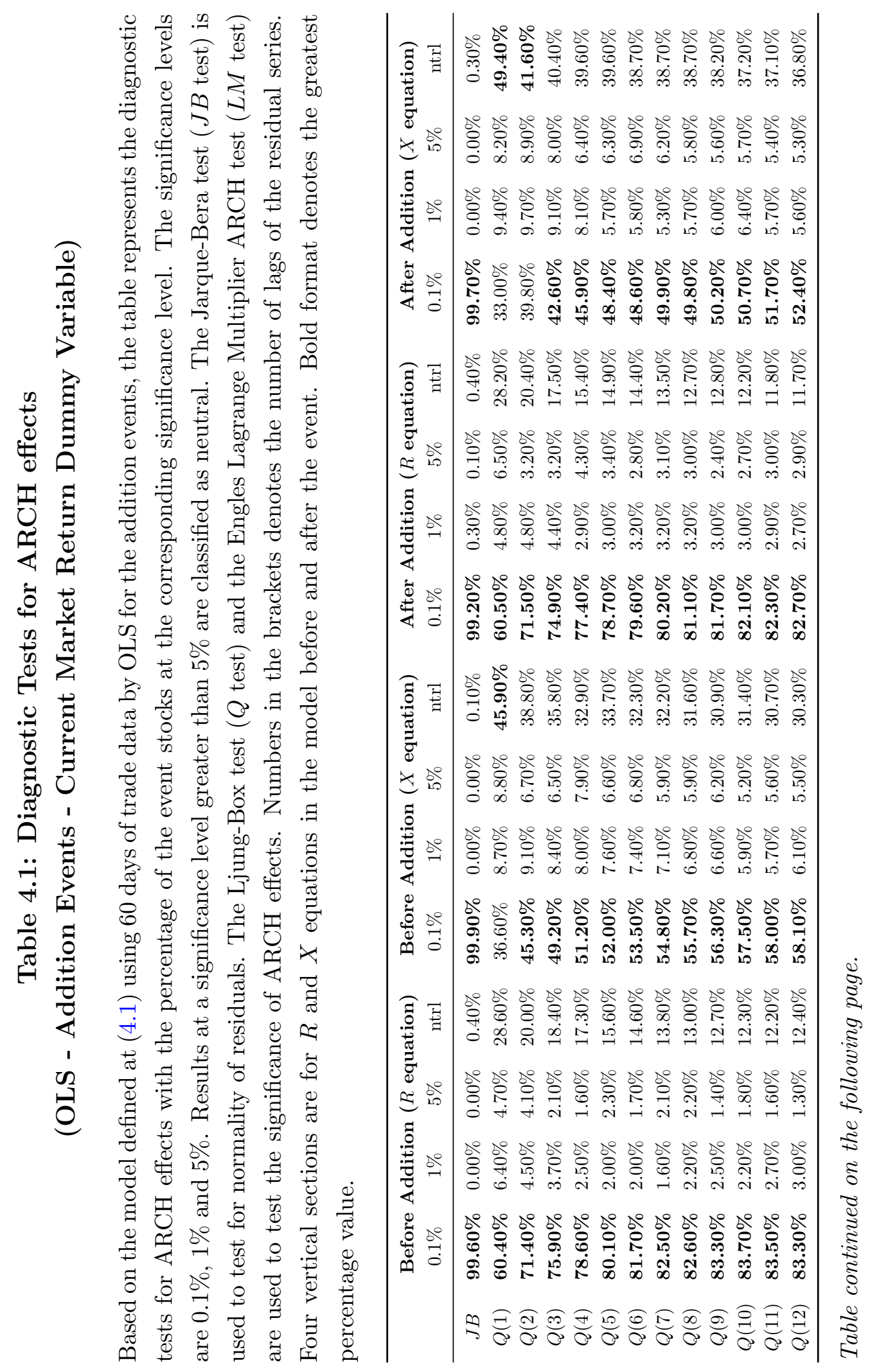




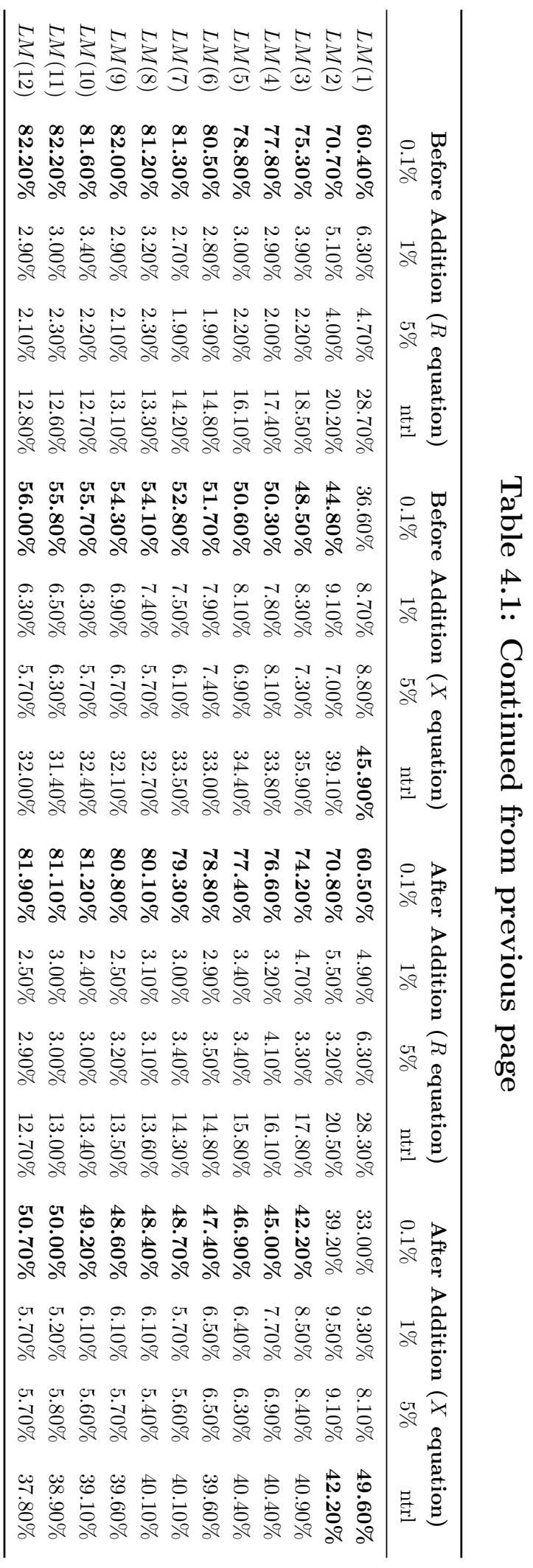




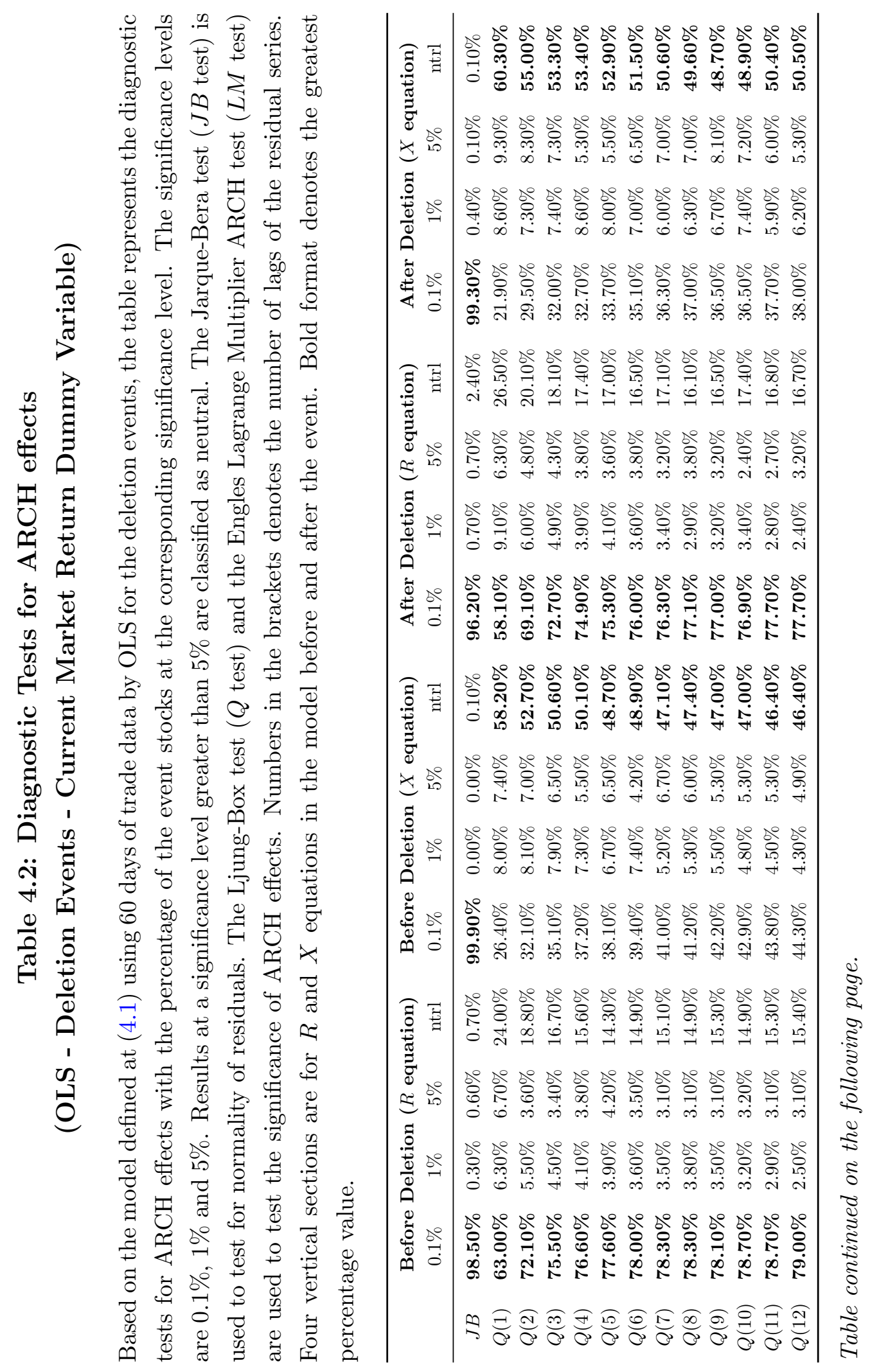




\begin{tabular}{|c|c|}
\hline 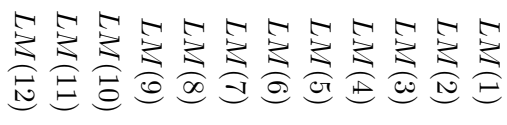 & \\
\hline 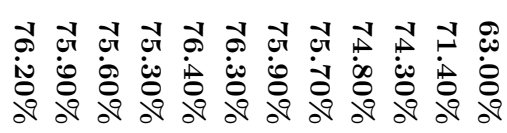 & 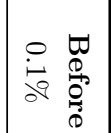 \\
\hline 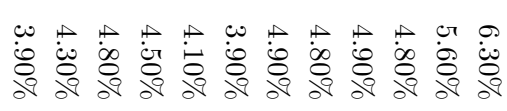 & 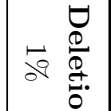 \\
\hline 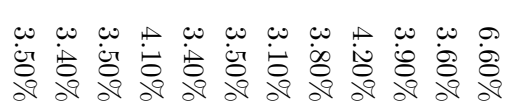 & $\begin{array}{cc}c & \widehat{A} \\
09 & 0 \\
0 & 8\end{array}$ \\
\hline 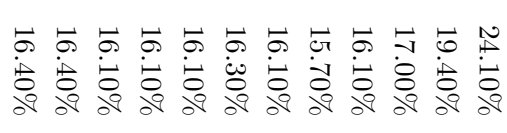 & 灵总 \\
\hline 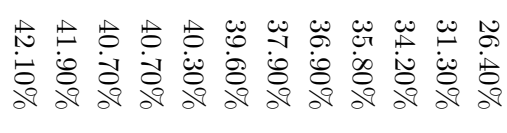 & $\mid \begin{array}{cc}0 & \mathbb{0} \\
\dot{\vec{J}} & 0 \\
0 & 0 \\
0 & 0 \\
0\end{array}$ \\
\hline 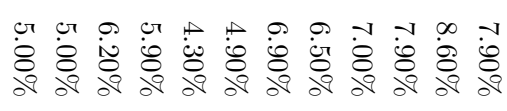 & 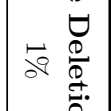 \\
\hline 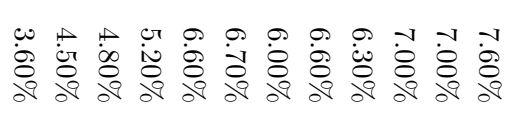 & $\nexists$ \\
\hline 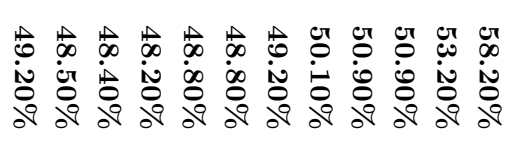 & 焉惫 \\
\hline 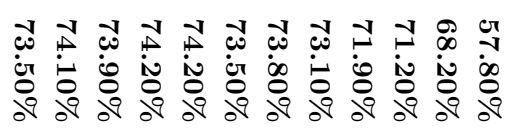 & 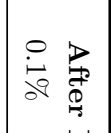 \\
\hline 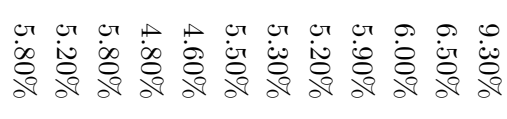 & 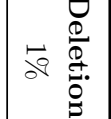 \\
\hline 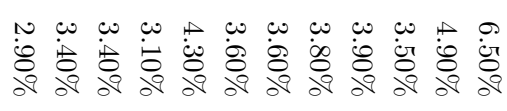 & \\
\hline 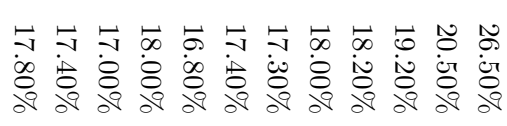 & z \\
\hline 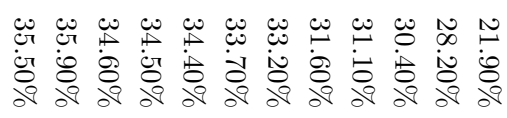 & 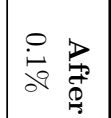 \\
\hline 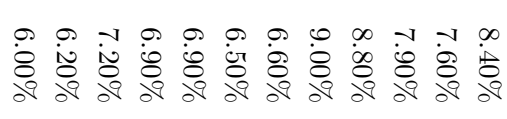 & 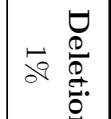 \\
\hline 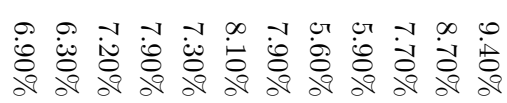 & वु $\widehat{A}$ \\
\hline 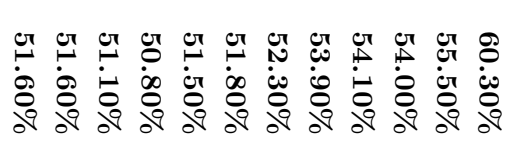 & $=$ Е. \\
\hline
\end{tabular}




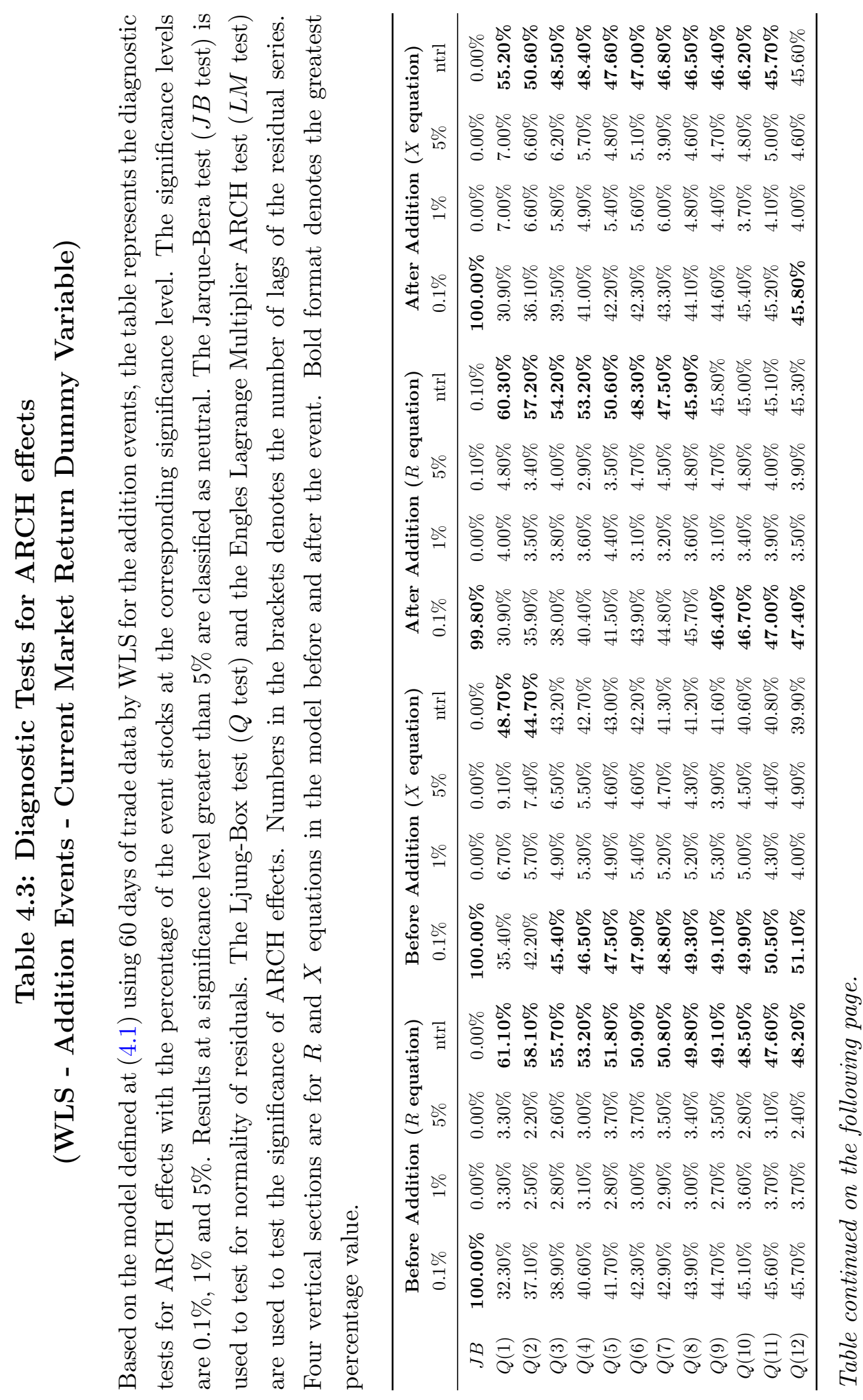




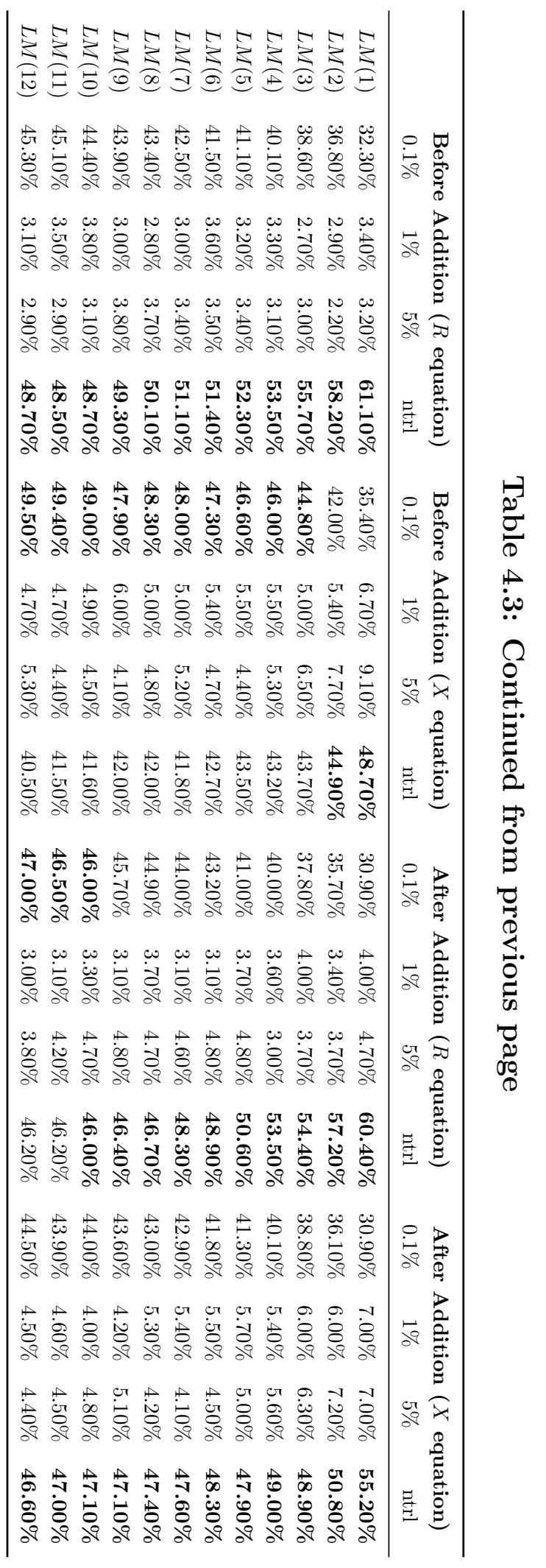




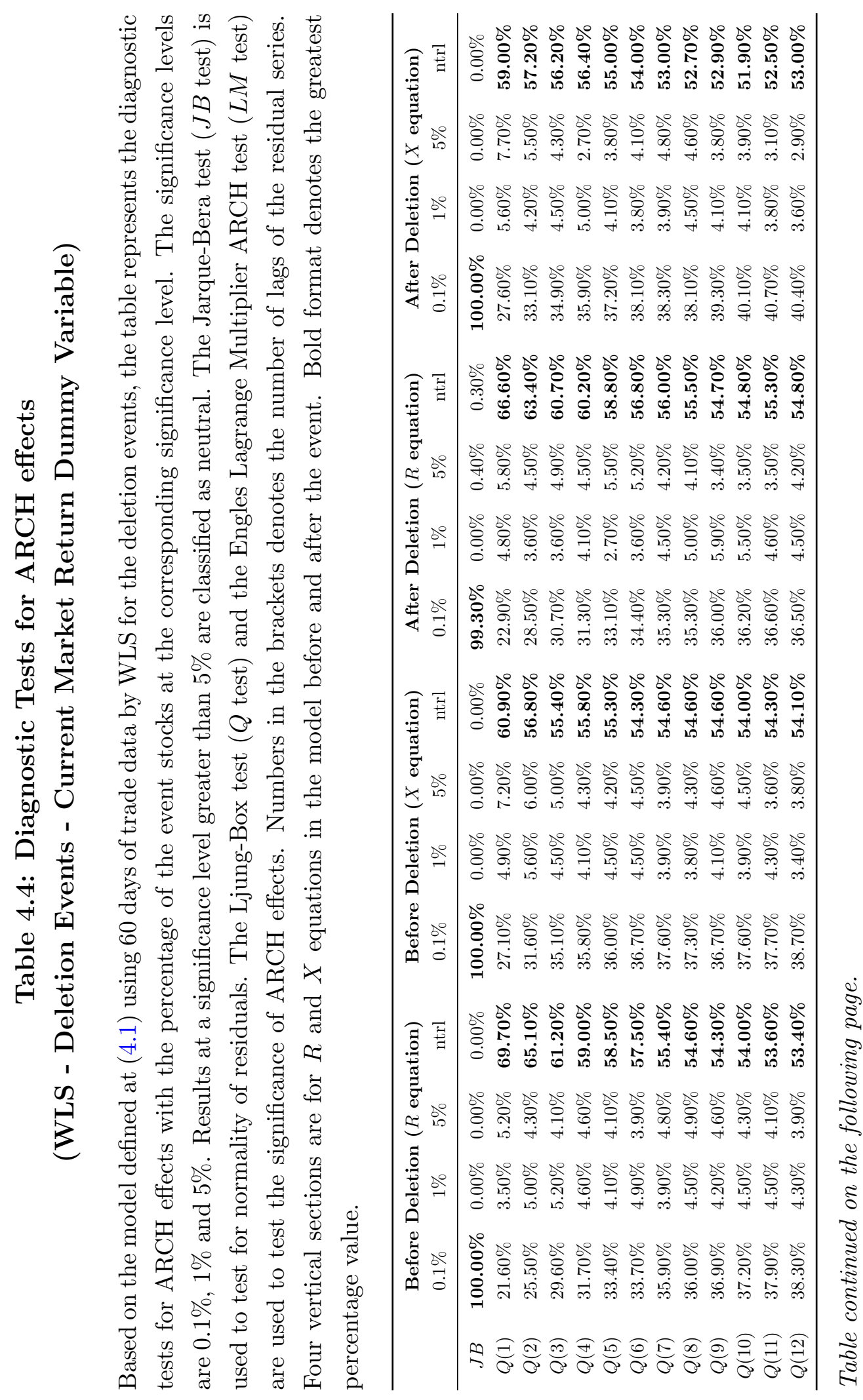




\begin{tabular}{|c|c|}
\hline 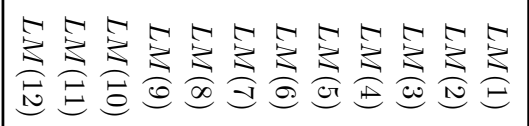 & \\
\hline 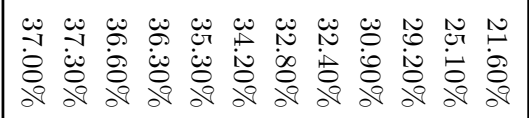 & 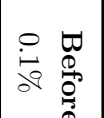 \\
\hline 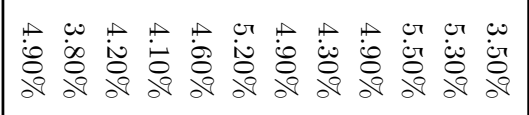 & aq \\
\hline 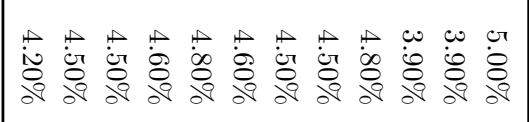 & 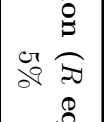 \\
\hline 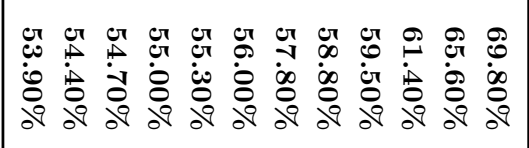 & 量量 \\
\hline 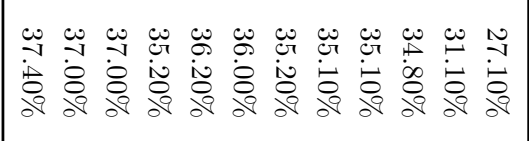 & 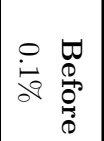 \\
\hline 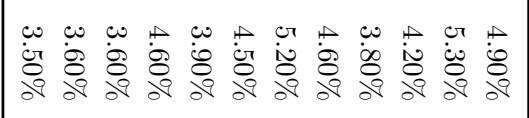 & 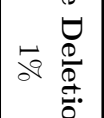 \\
\hline 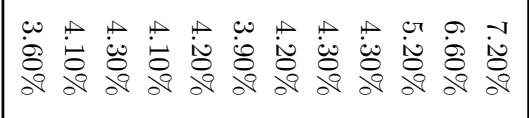 & 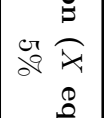 \\
\hline 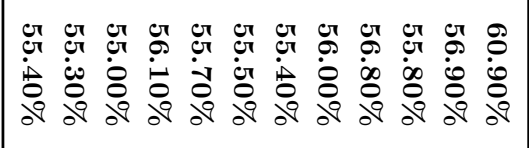 & 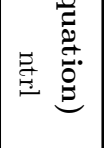 \\
\hline 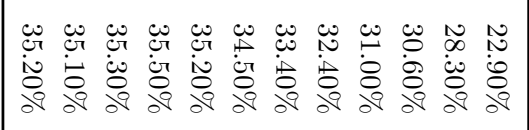 & 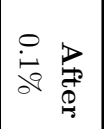 \\
\hline 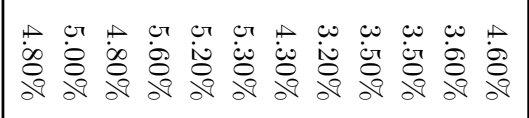 & 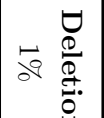 \\
\hline 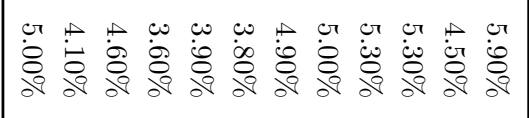 & 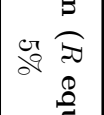 \\
\hline 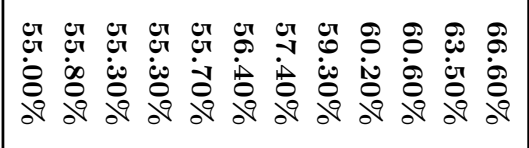 & 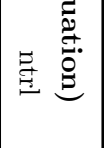 \\
\hline 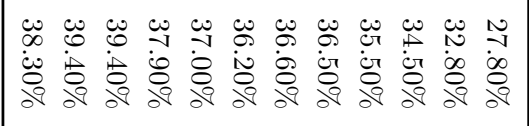 & 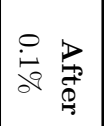 \\
\hline 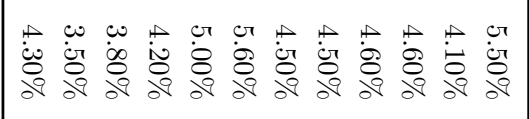 & 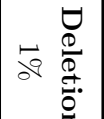 \\
\hline 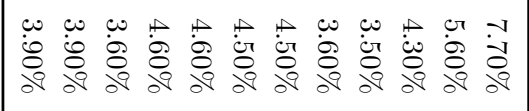 & 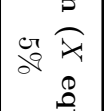 \\
\hline 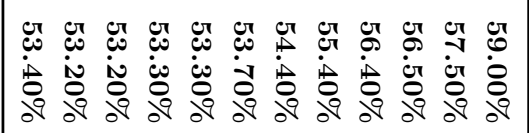 & 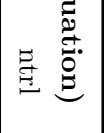 \\
\hline
\end{tabular}




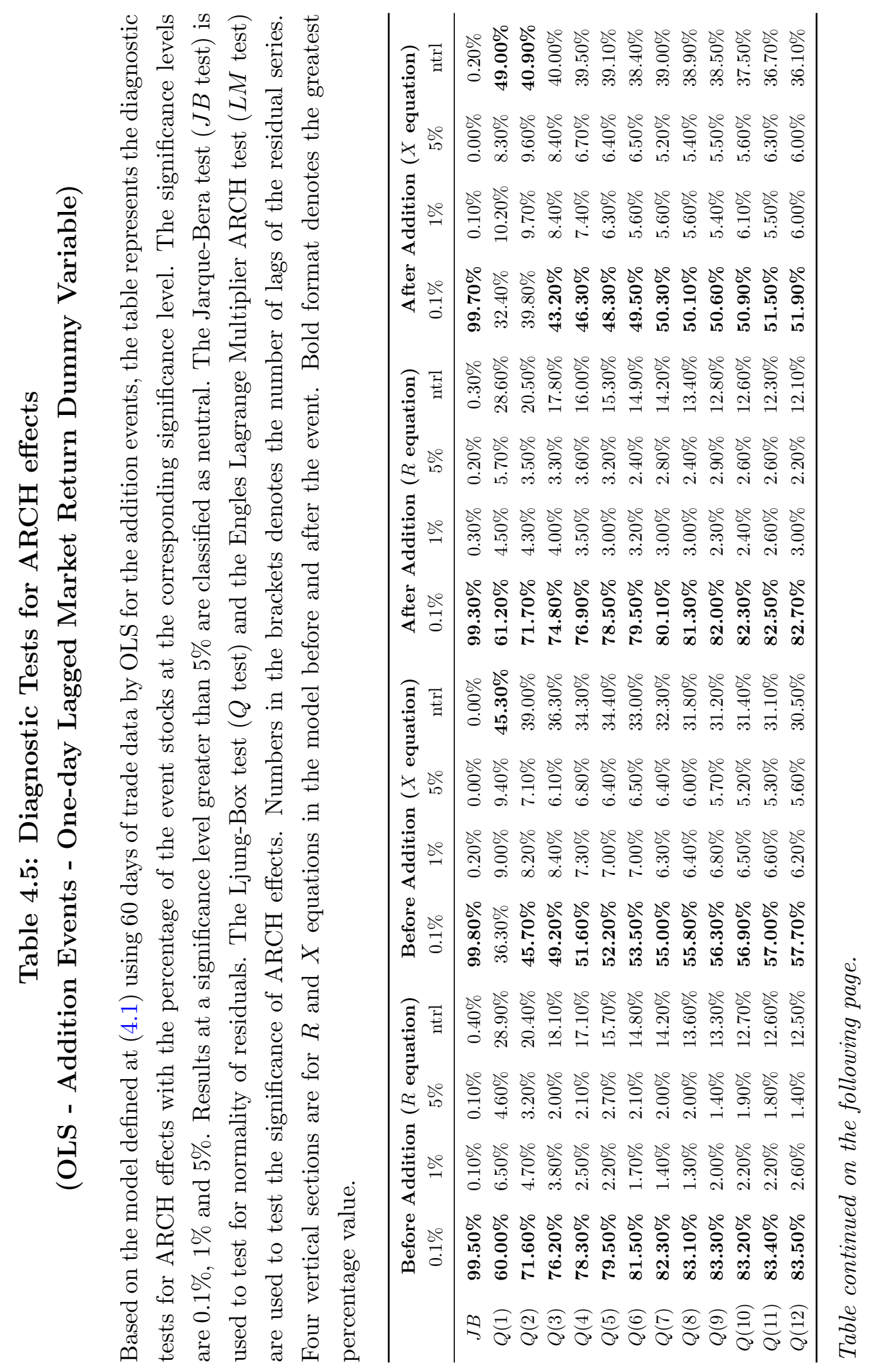




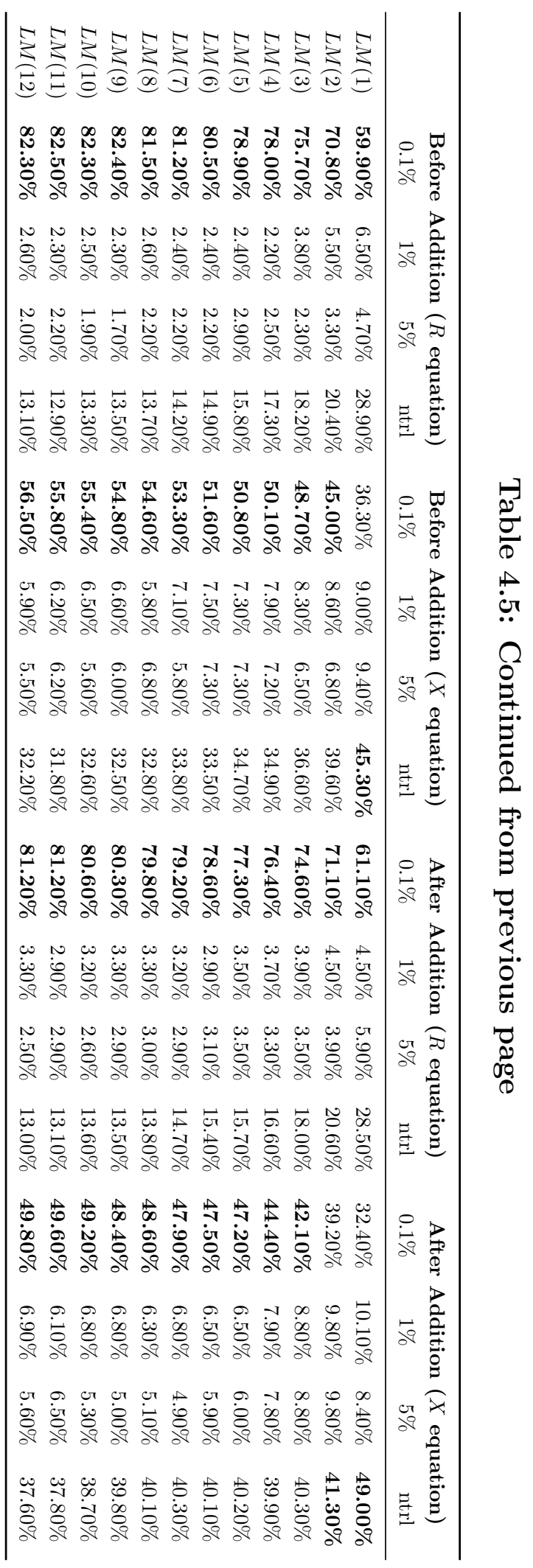




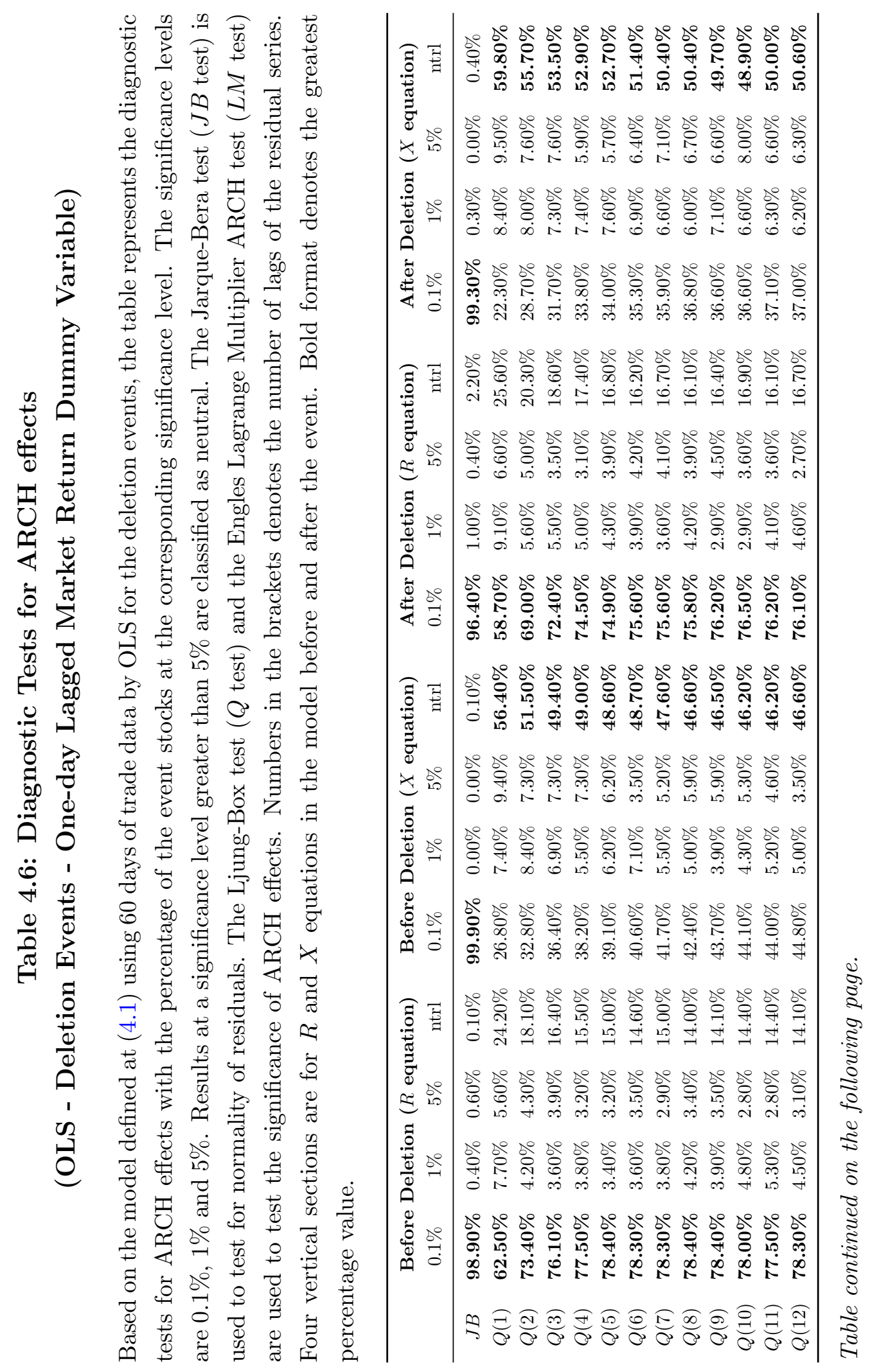




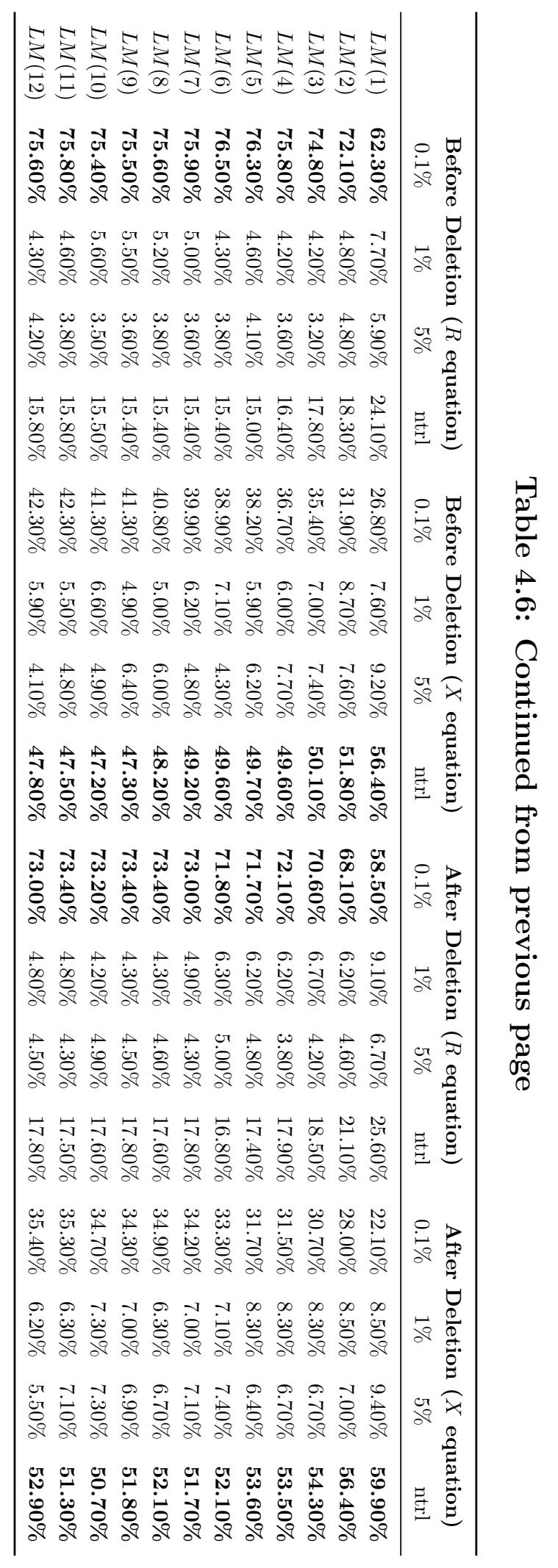




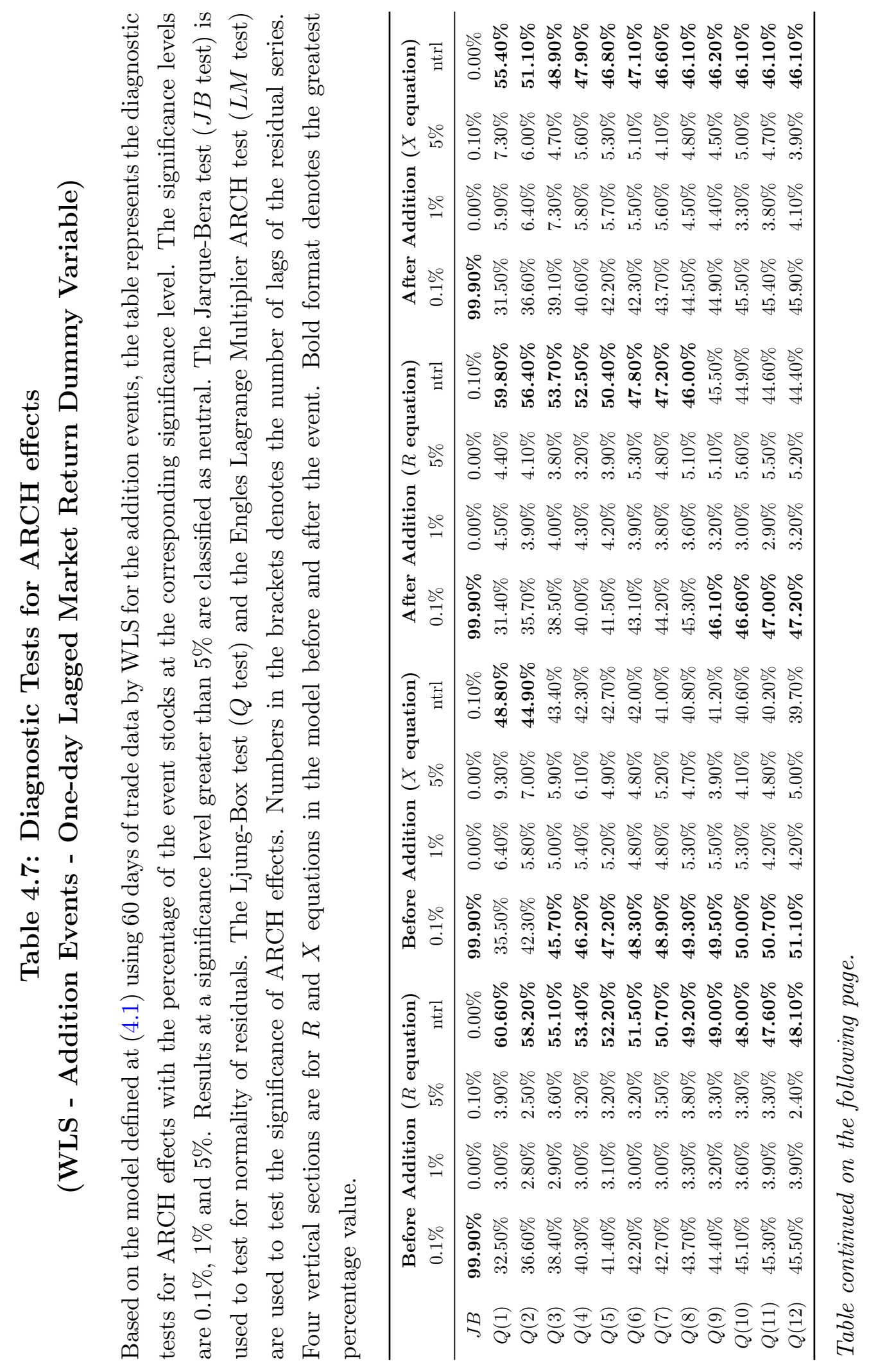




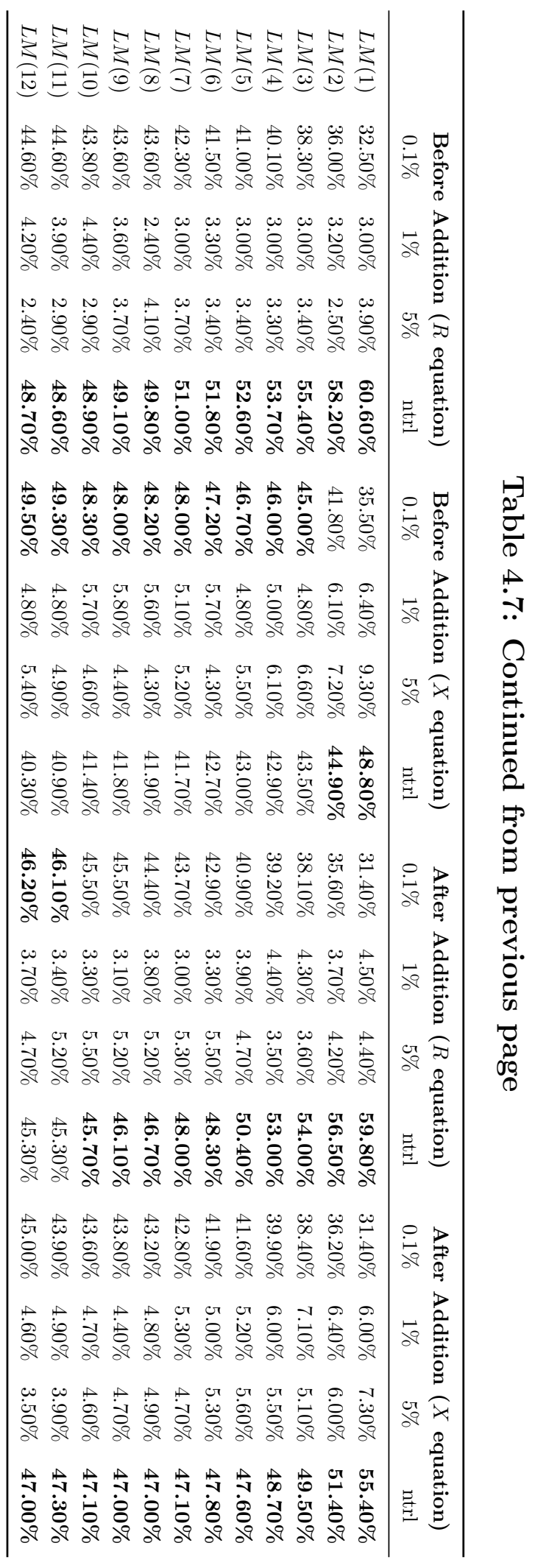




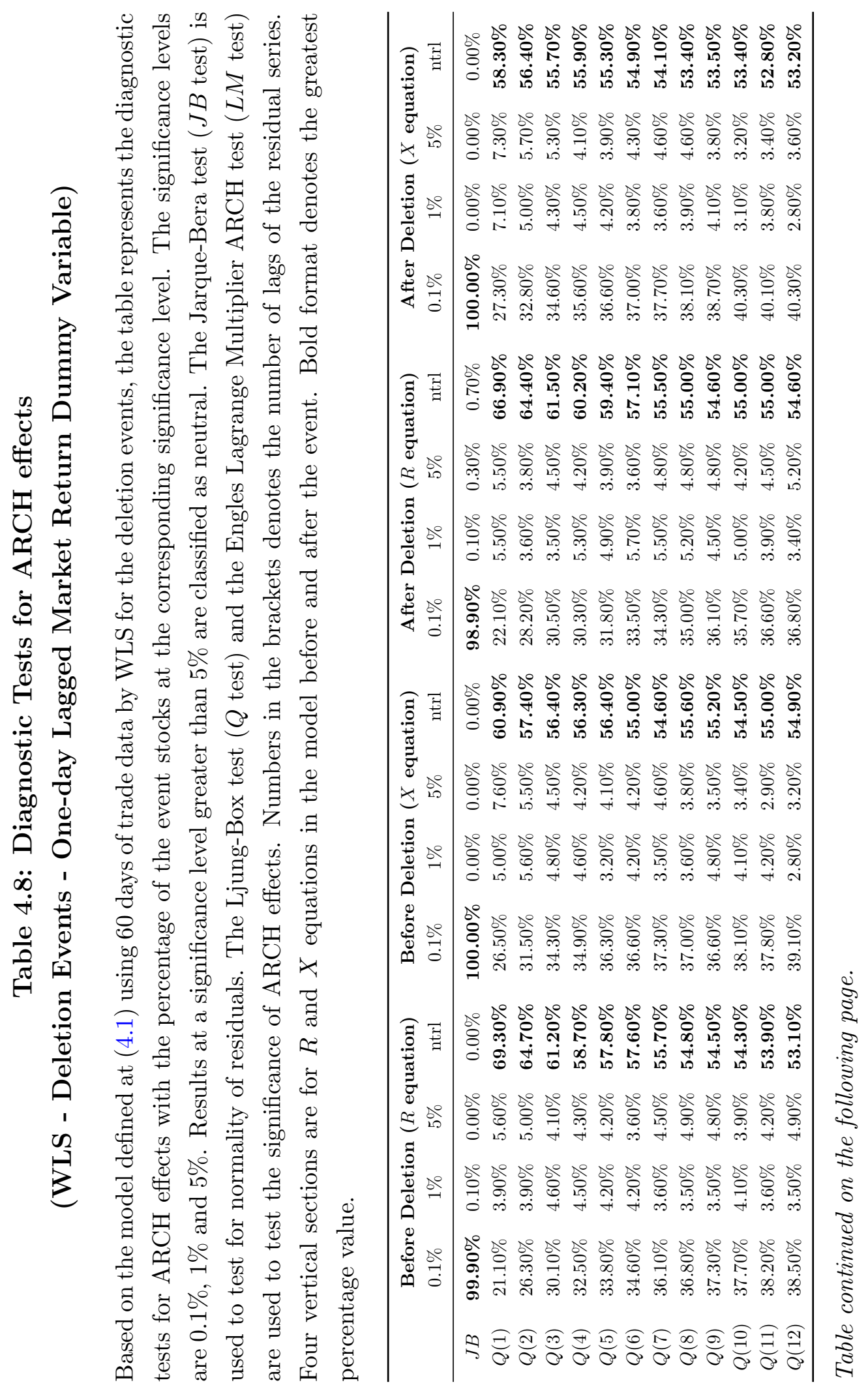




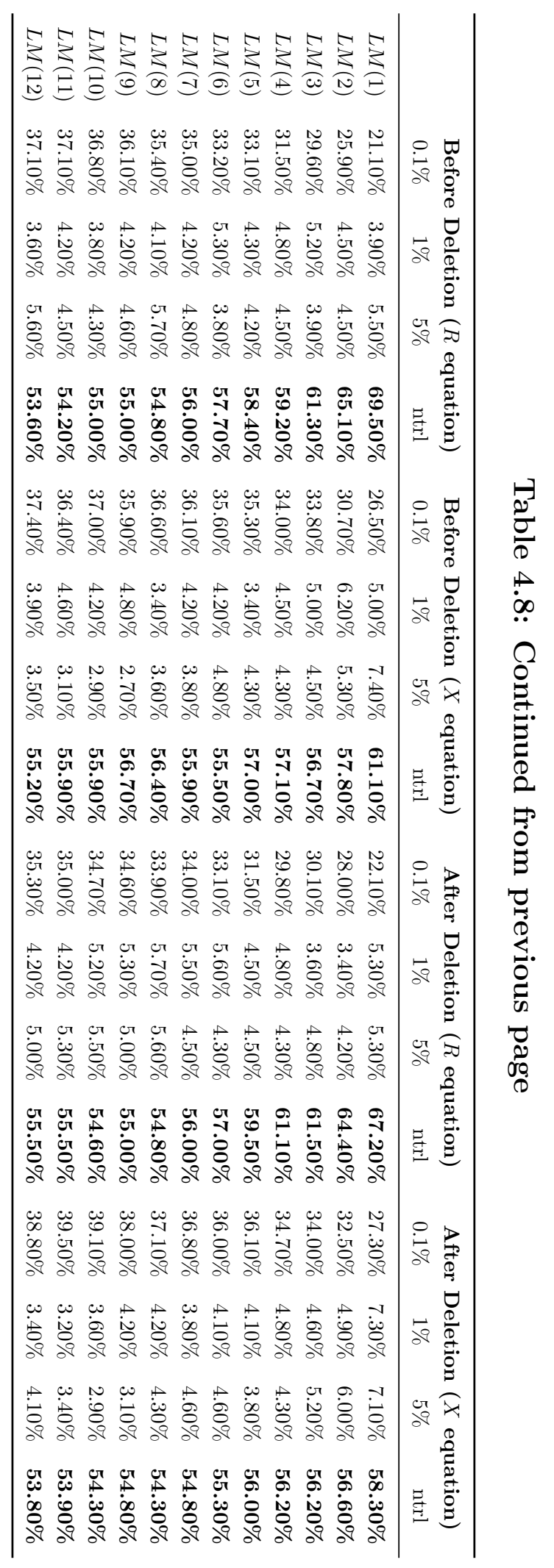




\section{Table 4.9: P-Value for Tests of Model Parameters (OLS - Dummy Variable of Current Market Return)}

The table is based on the model defined at (4.1) using 60 days of trade data by OLS interacted with a dummy variable of current market return. The tests for individual parameters and sums are computed as described in Section 2.3.3. The cell entries show the estimated parameters interacted with the corresponding dummy variable and their probabilities (p-val.), respectively. Differences are calculated by absolute value of model parameters between after and before both events. $D_{m, t}$ is the dummy variable denoted 1 if the market return on that day is positive or 0 if it is non-positive. Bold format denotes significance at the $1 \%$ level.

\begin{tabular}{|c|c|c|c|c|c|c|c|c|c|c|c|c|}
\hline & \multicolumn{6}{|c|}{ Addition } & \multicolumn{6}{|c|}{ Deletion } \\
\hline & \multicolumn{2}{|c|}{ Before } & \multicolumn{2}{|c|}{ After } & \multicolumn{2}{|c|}{ Difference } & \multicolumn{2}{|c|}{ Before } & \multicolumn{2}{|c|}{ After } & \multicolumn{2}{|c|}{ Difference } \\
\hline & Coef. & p-val. & Coef. & p-val. & Coef. & p-val. & Coef. & p-val. & Coef. & p-val. & Coef. & p-val. \\
\hline$D_{m}, R$ & 0.056 & 0.000 & 0.102 & 0.000 & 0.046 & 0.021 & 0.277 & 0.000 & 0.143 & 0.009 & -0.134 & 0.044 \\
\hline$\alpha_{1 \_} D_{m}$ & 0.000 & 0.945 & 0.002 & 0.507 & 0.001 & 0.668 & -0.005 & 0.178 & 0.003 & 0.460 & -0.002 & 0.731 \\
\hline$\alpha_{2-} D_{m}$ & -0.005 & 0.013 & -0.004 & 0.067 & -0.001 & 0.780 & -0.003 & 0.364 & -0.003 & 0.472 & -0.001 & 0.921 \\
\hline$\alpha_{3-} D_{m}$ & -0.001 & 0.581 & -0.005 & 0.024 & 0.004 & 0.197 & 0.002 & 0.590 & 0.001 & 0.741 & 0.000 & 0.953 \\
\hline$\alpha_{4-} D_{m}$ & 0.001 & 0.766 & 0.001 & 0.725 & 0.000 & 0.968 & -0.002 & 0.533 & 0.001 & 0.759 & -0.001 & 0.879 \\
\hline$\alpha_{5-} D_{m}$ & 0.000 & 0.929 & 0.003 & 0.140 & 0.003 & 0.279 & 0.000 & 0.972 & 0.001 & 0.813 & 0.001 & 0.867 \\
\hline$\beta_{0-} D_{m}$ & -0.003 & 0.876 & 0.019 & 0.036 & 0.016 & 0.459 & -0.052 & 0.014 & 0.032 & 0.480 & -0.020 & 0.684 \\
\hline$\beta_{1-} D_{m}$ & -0.024 & 0.433 & -0.002 & 0.830 & -0.022 & 0.475 & 0.033 & 0.068 & 0.432 & 0.034 & 0.399 & 0.051 \\
\hline$\beta_{2-} D_{m}$ & 0.042 & 0.144 & -0.021 & 0.010 & -0.021 & 0.482 & 0.045 & 0.005 & -0.212 & 0.310 & 0.167 & 0.426 \\
\hline$\beta_{3-} D_{m}$ & 0.017 & 0.499 & -0.018 & 0.043 & 0.001 & 0.960 & -0.021 & 0.308 & -1.451 & 0.038 & 1.430 & 0.041 \\
\hline$\beta_{4-} D_{m}$ & -0.042 & 0.125 & -0.005 & 0.541 & -0.037 & 0.187 & -0.003 & 0.850 & 0.125 & 0.061 & 0.122 & 0.075 \\
\hline$\beta_{5-} D_{m}$ & -0.009 & 0.547 & 0.016 & 0.092 & 0.007 & 0.698 & -0.007 & 0.636 & -0.037 & 0.283 & 0.030 & 0.412 \\
\hline$D_{m}, X$ & 0.357 & 0.000 & 0.445 & 0.000 & 0.089 & 0.041 & 0.879 & 0.000 & 0.637 & 0.000 & -0.242 & 0.026 \\
\hline$\gamma_{1-} D_{m}$ & 0.002 & 0.721 & -0.006 & 0.309 & 0.004 & 0.669 & -0.011 & 0.141 & -0.004 & 0.630 & -0.007 & 0.561 \\
\hline$\gamma_{2-} D_{m}$ & -0.001 & 0.887 & -0.005 & 0.424 & 0.004 & 0.661 & -0.019 & 0.017 & 0.005 & 0.566 & -0.013 & 0.268 \\
\hline$\gamma_{3-} D_{m}$ & -0.009 & 0.171 & -0.003 & 0.646 & -0.006 & 0.471 & -0.006 & 0.338 & 0.006 & 0.406 & 0.000 & 0.999 \\
\hline$\gamma_{4-} D_{m}$ & -0.006 & 0.275 & -0.003 & 0.643 & -0.004 & 0.661 & -0.006 & 0.381 & 0.001 & 0.898 & -0.005 & 0.622 \\
\hline$\gamma_{5-} D_{m}$ & 0.011 & 0.056 & 0.000 & 0.926 & -0.010 & 0.157 & -0.004 & 0.548 & 0.007 & 0.300 & 0.004 & 0.694 \\
\hline$\delta_{1-} D_{m}$ & 0.002 & 0.244 & 0.002 & 0.403 & 0.000 & 0.879 & -0.003 & 0.438 & -0.005 & 0.195 & 0.002 & 0.645 \\
\hline$\delta_{2-} D_{m}$ & -0.004 & 0.034 & 0.001 & 0.660 & -0.003 & 0.236 & 0.002 & 0.540 & 0.001 & 0.785 & -0.001 & 0.845 \\
\hline$\delta_{3-} D_{m}$ & 0.004 & 0.073 & -0.004 & 0.035 & 0.000 & 0.900 & 0.004 & 0.143 & -0.003 & 0.425 & -0.001 & 0.812 \\
\hline$\delta_{4-} D_{m}$ & 0.002 & 0.256 & 0.000 & 0.911 & -0.002 & 0.467 & 0.002 & 0.563 & -0.003 & 0.462 & 0.001 & 0.855 \\
\hline$\delta_{5-} D_{m}$ & -0.004 & 0.058 & -0.001 & 0.640 & -0.003 & 0.340 & -0.001 & 0.858 & 0.003 & 0.388 & 0.003 & 0.564 \\
\hline$A_{-} D_{m}$ & -0.005 & 0.352 & -0.004 & 0.510 & -0.001 & 0.891 & -0.009 & 0.399 & 0.004 & 0.755 & -0.005 & 0.755 \\
\hline$B \_D_{m}$ & -0.019 & 0.396 & -0.010 & 0.549 & -0.009 & 0.754 & -0.005 & 0.887 & -1.112 & 0.081 & 1.108 & 0.082 \\
\hline$\Gamma \_D_{m}$ & -0.003 & 0.857 & -0.017 & 0.301 & 0.014 & 0.552 & -0.045 & 0.028 & 0.016 & 0.501 & -0.030 & 0.341 \\
\hline$\Delta_{-} D_{m}$ & 0.001 & 0.853 & -0.002 & 0.556 & 0.001 & 0.781 & 0.005 & 0.387 & -0.007 & 0.337 & 0.002 & 0.861 \\
\hline
\end{tabular}




\section{Table 4.10: P-Value for Tests of Model Parameters (WLS - Dummy Variable of Current Market Return)}

The table is based on the model defined at (4.1) using 60 days of trade data by WLS interacted with a dummy variable of current market return. The tests for individual parameters and sums are computed as described in Section 2.3.3. The cell entries show the estimated parameters interacted with the corresponding dummy variable and their probabilities (p-val.), respectively. Differences are calculated by absolute value of model parameters between after and before both events. $D_{m, t}$ is the dummy variable denoted 1 if the market return on that day is positive or 0 if it is non-positive. Bold format denotes significance at the $1 \%$ level.

\begin{tabular}{|c|c|c|c|c|c|c|c|c|c|c|c|c|}
\hline & \multicolumn{6}{|c|}{ Addition } & \multicolumn{6}{|c|}{ Deletion } \\
\hline & \multicolumn{2}{|c|}{ Before } & \multicolumn{2}{|c|}{ After } & \multicolumn{2}{|c|}{ Difference } & \multicolumn{2}{|c|}{ Before } & \multicolumn{2}{|c|}{ After } & \multicolumn{2}{|c|}{ Difference } \\
\hline & Coef. & p-val. & Coef. & p-val. & Coef. & p-val. & Coef. & p-val. & Coef. & p-val. & Coef. & p-val. \\
\hline$D_{m}, R$ & 0.002 & 0.340 & 0.007 & 0.008 & 0.005 & 0.101 & 0.019 & 0.006 & -0.003 & 0.645 & -0.015 & 0.138 \\
\hline$\alpha_{1 \_} D_{m}$ & -0.003 & 0.097 & 0.004 & 0.081 & 0.001 & 0.781 & 0.001 & 0.722 & 0.002 & 0.579 & 0.001 & 0.872 \\
\hline$\alpha_{2-} D_{m}$ & -0.003 & 0.165 & -0.002 & 0.512 & -0.001 & 0.663 & 0.003 & 0.491 & -0.007 & 0.193 & 0.005 & 0.495 \\
\hline$\alpha_{3-} D_{m}$ & 0.000 & 0.981 & 0.009 & 0.024 & 0.009 & 0.042 & 0.010 & 0.003 & 0.001 & 0.790 & -0.009 & 0.086 \\
\hline$\alpha_{4-} D_{m}$ & 0.002 & 0.295 & 0.001 & 0.751 & -0.001 & 0.661 & 0.005 & 0.110 & -0.001 & 0.817 & -0.004 & 0.457 \\
\hline$\alpha_{5-} D_{m}$ & 0.002 & 0.350 & -0.001 & 0.687 & 0.000 & 0.961 & -0.006 & 0.060 & 0.003 & 0.473 & -0.003 & 0.580 \\
\hline$\beta_{0-} D_{m}$ & -0.010 & 0.370 & -0.089 & 0.011 & 0.079 & 0.029 & -0.022 & 0.361 & -0.053 & 0.346 & 0.031 & 0.610 \\
\hline$\beta_{1-} D_{m}$ & 0.002 & 0.841 & -0.008 & 0.375 & 0.006 & 0.696 & -0.025 & 0.107 & 0.077 & 0.074 & 0.052 & 0.258 \\
\hline$\beta_{2-} D_{m}$ & 0.007 & 0.390 & -0.006 & 0.614 & -0.001 & 0.930 & -0.006 & 0.673 & 0.089 & 0.012 & 0.083 & 0.032 \\
\hline$\beta_{3-} D_{m}$ & 0.004 & 0.668 & 0.011 & 0.340 & 0.007 & 0.673 & -0.036 & 0.018 & 0.057 & 0.211 & 0.020 & 0.672 \\
\hline$\beta_{4-} D_{m}$ & -0.012 & 0.300 & 0.024 & 0.020 & 0.012 & 0.422 & -0.004 & 0.765 & 0.031 & 0.389 & 0.027 & 0.484 \\
\hline$\beta_{5-} D_{m}$ & -0.010 & 0.382 & 0.093 & 0.004 & 0.083 & 0.017 & -0.011 & 0.450 & 0.038 & 0.205 & 0.027 & 0.424 \\
\hline$D_{m}, X$ & 0.097 & 0.000 & 0.098 & 0.000 & 0.000 & 0.994 & 0.163 & 0.000 & 0.108 & 0.000 & -0.055 & 0.113 \\
\hline$\gamma_{1-} D_{m}$ & 0.018 & 0.145 & 0.005 & 0.660 & -0.013 & 0.428 & 0.005 & 0.676 & -0.021 & 0.212 & 0.016 & 0.435 \\
\hline$\gamma_{2-} D_{m}$ & 0.009 & 0.362 & -0.001 & 0.879 & -0.008 & 0.562 & -0.001 & 0.934 & -0.016 & 0.295 & 0.015 & 0.414 \\
\hline$\gamma_{3-} D_{m}$ & 0.002 & 0.873 & 0.016 & 0.212 & 0.013 & 0.506 & 0.001 & 0.924 & 0.004 & 0.751 & 0.003 & 0.837 \\
\hline$\gamma_{4-} D_{m}$ & 0.006 & 0.525 & 0.021 & 0.075 & 0.015 & 0.336 & 0.002 & 0.833 & -0.017 & 0.236 & 0.015 & 0.389 \\
\hline$\gamma_{5-} D_{m}$ & 0.018 & 0.151 & 0.014 & 0.093 & -0.005 & 0.757 & 0.000 & 0.962 & 0.053 & 0.117 & 0.053 & 0.130 \\
\hline$\delta_{1-} D_{m}$ & 0.004 & 0.138 & 0.000 & 0.903 & -0.003 & 0.352 & 0.009 & 0.031 & 0.010 & 0.023 & 0.001 & 0.908 \\
\hline$\delta_{2-} D_{m}$ & -0.003 & 0.209 & 0.002 & 0.257 & -0.001 & 0.850 & 0.002 & 0.644 & -0.007 & 0.121 & 0.005 & 0.403 \\
\hline$\delta_{3-} D_{m}$ & 0.005 & 0.148 & -0.003 & 0.097 & -0.002 & 0.655 & -0.003 & 0.324 & 0.007 & 0.069 & 0.004 & 0.469 \\
\hline$\delta_{4-} D_{m}$ & 0.003 & 0.346 & 0.002 & 0.333 & -0.001 & 0.797 & -0.006 & 0.107 & -0.001 & 0.722 & -0.004 & 0.433 \\
\hline$\delta_{5-} D_{m}$ & -0.009 & 0.054 & -0.002 & 0.299 & -0.007 & 0.189 & -0.002 & 0.497 & -0.010 & 0.094 & 0.008 & 0.264 \\
\hline$A_{-} D_{m}$ & -0.003 & 0.615 & 0.011 & 0.071 & 0.009 & 0.278 & 0.013 & 0.153 & -0.002 & 0.883 & -0.012 & 0.465 \\
\hline$B \_D_{m}$ & -0.018 & 0.380 & 0.025 & 0.378 & 0.007 & 0.829 & -0.105 & 0.008 & 0.239 & 0.019 & 0.134 & 0.220 \\
\hline$\Gamma_{-} D_{m}$ & 0.055 & 0.066 & 0.054 & 0.045 & -0.001 & 0.981 & 0.007 & 0.796 & 0.004 & 0.923 & -0.003 & 0.957 \\
\hline$\Delta_{-} D_{m}$ & 0.000 & 0.958 & -0.001 & 0.831 & 0.001 & 0.916 & 0.000 & 0.965 & -0.001 & 0.931 & 0.000 & 0.968 \\
\hline
\end{tabular}




\section{Table 4.11: P-Value for Tests of Model Parameters (GARCH - Dummy Variable of Current Market Return)}

The table is based on the model defined at (4.2) using 60 days of trade data by GARCH $(1,1)$ interacted with a dummy variable of current market return. The tests for individual parameters and sums are computed as described in Section 2.3.3. The cell entries show the estimated parameters interacted with the corresponding dummy variable and their probabilities (p-val.), respectively. Differences are calculated by absolute value of model parameters between after and before both events. $D_{m, t}$ is the dummy variable denoted 1 if the market return on that day is positive or 0 if it is non-positive. Bold format denotes significance at the $1 \%$ level.

\begin{tabular}{|c|c|c|c|c|c|c|c|c|c|c|c|c|}
\hline & \multicolumn{6}{|c|}{ Addition } & \multicolumn{6}{|c|}{ Deletion } \\
\hline & \multicolumn{2}{|c|}{ Before } & \multicolumn{2}{|c|}{ After } & \multicolumn{2}{|c|}{ Difference } & \multicolumn{2}{|c|}{ Before } & \multicolumn{2}{|c|}{ After } & \multicolumn{2}{|c|}{ Difference } \\
\hline & Coef. & p-val. & Coef. & p-val. & Coef. & p-val. & Coef. & p-val. & Coef. & p-val. & Coef. & p-val. \\
\hline$D_{m}, R$ & 0.053 & 0.018 & 0.036 & 0.941 & -0.017 & 0.973 & 0.083 & 0.366 & 0.077 & 0.895 & -0.006 & 0.992 \\
\hline$\alpha_{1} D_{m}$ & -0.002 & 0.436 & 0.000 & 1.000 & -0.002 & 0.995 & -0.007 & 0.286 & 0.004 & 0.981 & -0.003 & 0.988 \\
\hline$\alpha_{2} D_{m}$ & -0.004 & 0.102 & -0.006 & 0.991 & 0.001 & 0.998 & -0.008 & 0.160 & -0.002 & 0.992 & -0.005 & 0.983 \\
\hline$\alpha_{3} D_{m}$ & 0.000 & 0.967 & -0.005 & 0.996 & 0.004 & 0.996 & 0.001 & 0.953 & -0.001 & 0.997 & 0.001 & 0.999 \\
\hline$\alpha_{4} D_{m}$ & -0.002 & 0.699 & -0.001 & 0.994 & -0.001 & 0.992 & -0.002 & 0.809 & 0.001 & 0.991 & 0.000 & 0.998 \\
\hline$\alpha_{5} D_{m}$ & 0.001 & 0.844 & 0.003 & 0.939 & 0.002 & 0.955 & 0.004 & 0.360 & -0.001 & 0.992 & -0.003 & 0.976 \\
\hline$\beta_{0} D_{m}$ & -0.028 & 0.300 & 0.040 & 0.972 & 0.012 & 0.992 & -0.047 & 0.652 & 0.033 & 0.969 & -0.014 & 0.987 \\
\hline$\beta_{1 \_} D_{m}$ & -0.014 & 0.782 & -0.011 & 0.982 & -0.003 & 0.995 & 0.005 & 0.966 & 0.502 & 0.842 & 0.497 & 0.843 \\
\hline$\beta_{2} D_{m}$ & 0.032 & 0.379 & -0.005 & 0.997 & -0.027 & 0.980 & 0.038 & 0.792 & -0.171 & 0.983 & 0.133 & 0.987 \\
\hline$\beta_{3-} D_{m}$ & 0.000 & 0.993 & -0.021 & 0.986 & 0.020 & 0.987 & -0.014 & 0.957 & -1.472 & 0.501 & 1.458 & 0.509 \\
\hline$\beta_{4} D_{m}$ & -0.005 & 0.899 & -0.003 & 0.998 & -0.002 & 0.999 & -0.010 & 0.972 & 0.082 & 0.985 & 0.071 & 0.987 \\
\hline$\beta_{5-} D_{m}$ & 0.011 & 0.767 & 0.009 & 0.981 & -0.002 & 0.996 & -0.031 & 0.659 & -0.018 & 0.999 & -0.013 & 0.999 \\
\hline$D_{m}, X$ & 0.253 & 0.000 & 0.377 & 0.000 & 0.124 & 0.008 & 0.757 & 0.000 & 0.624 & 0.000 & -0.132 & 0.454 \\
\hline$\gamma_{1-} D_{m}$ & 0.015 & 0.002 & -0.001 & 0.930 & -0.014 & 0.106 & -0.009 & 0.513 & -0.007 & 0.618 & -0.002 & 0.901 \\
\hline$\gamma_{2 \_} D_{m}$ & 0.014 & 0.008 & -0.015 & 0.072 & 0.000 & 0.965 & -0.021 & 0.240 & 0.012 & 0.464 & -0.009 & 0.713 \\
\hline$\gamma_{3-} D_{m}$ & 0.008 & 0.105 & -0.002 & 0.818 & -0.006 & 0.579 & -0.015 & 0.295 & 0.006 & 0.734 & -0.008 & 0.715 \\
\hline$\gamma_{4-} D_{m}$ & 0.000 & 0.999 & 0.006 & 0.526 & 0.006 & 0.581 & 0.001 & 0.949 & 0.000 & 0.985 & -0.001 & 0.980 \\
\hline$\gamma_{5} D_{m}$ & 0.015 & 0.004 & -0.009 & 0.256 & -0.005 & 0.577 & 0.004 & 0.675 & 0.011 & 0.546 & 0.007 & 0.750 \\
\hline$\delta_{1 \_} D_{m}$ & 0.002 & 0.404 & 0.007 & 0.081 & 0.005 & 0.286 & 0.009 & 0.769 & -0.006 & 0.559 & -0.003 & 0.934 \\
\hline$\delta_{2}-D_{m}$ & -0.004 & 0.037 & -0.001 & 0.786 & -0.003 & 0.514 & 0.004 & 0.884 & 0.004 & 0.696 & 0.000 & 0.995 \\
\hline$\delta_{3-} D_{m}$ & 0.000 & 0.902 & 0.000 & 0.972 & 0.000 & 0.989 & 0.010 & 0.663 & -0.004 & 0.871 & -0.006 & 0.867 \\
\hline$\delta_{4-} D_{m}$ & -0.001 & 0.574 & -0.005 & 0.337 & 0.004 & 0.512 & 0.000 & 0.996 & -0.005 & 0.539 & 0.005 & 0.905 \\
\hline$\delta_{5-} D_{m}$ & -0.002 & 0.385 & -0.001 & 0.893 & -0.001 & 0.796 & -0.003 & 0.939 & 0.005 & 0.564 & 0.003 & 0.950 \\
\hline$A_{-} D_{m}$ & -0.008 & 0.483 & -0.008 & 0.996 & 0.001 & 1.000 & -0.012 & 0.649 & 0.001 & 0.998 & -0.011 & 0.979 \\
\hline$B \_D_{m}$ & -0.005 & 0.916 & 0.010 & 0.989 & 0.005 & 0.995 & -0.060 & 0.930 & -1.044 & 0.916 & 0.983 & 0.921 \\
\hline$\Gamma_{-} D_{m}$ & 0.052 & 0.000 & -0.021 & 0.351 & -0.031 & 0.238 & -0.040 & 0.340 & 0.023 & 0.648 & -0.017 & 0.794 \\
\hline$\Delta_{-} D_{m}$ & -0.005 & 0.160 & 0.000 & 0.970 & -0.005 & 0.617 & 0.020 & 0.508 & -0.006 & 0.840 & -0.014 & 0.748 \\
\hline
\end{tabular}


Table 4.12: P-Value for Tests of Model Parameters (OLS - Dummy Variable of One-day Lagged Market Return)

The table is based on the model defined at (4.1) using 60 days of trade data by OLS interacted with a dummy variable of one-day lagged market return. The tests for individual parameters and sums are computed as described in Section 2.3.3. The cell entries show the estimated parameters interacted with the corresponding dummy variable and their probabilities (p-val.), respectively. Differences are calculated by absolute value of model parameters between after and before both events. $D_{\text {mlag, } t}$ is the dummy variable denoted 1 if the market return on that day is positive or 0 if it is non-positive. Bold format denotes significance at the $1 \%$ level.

\begin{tabular}{|c|c|c|c|c|c|c|c|c|c|c|c|c|}
\hline & \multicolumn{6}{|c|}{ Addition } & \multicolumn{6}{|c|}{ Deletion } \\
\hline & \multicolumn{2}{|c|}{ Before } & \multicolumn{2}{|c|}{ After } & \multicolumn{2}{|c|}{ Difference } & \multicolumn{2}{|c|}{ Before } & \multicolumn{2}{|c|}{ After } & \multicolumn{2}{|c|}{ Difference } \\
\hline & Coef. & p-val. & Coef. & p-val. & Coef. & p-val. & Coef. & p-val. & Coef. & p-val. & Coef. & p-val. \\
\hline$D_{m} \operatorname{lag}, R$ & 0.007 & 0.602 & 0.000 & 0.997 & -0.007 & 0.754 & -0.031 & 0.476 & -0.155 & 0.006 & 0.124 & 0.079 \\
\hline$\alpha_{1-} D_{m} l a g$ & 0.000 & 0.978 & -0.003 & 0.267 & 0.003 & 0.431 & -0.001 & 0.787 & -0.004 & 0.296 & 0.003 & 0.558 \\
\hline$\alpha_{2-} D_{m} l a g$ & -0.001 & 0.553 & -0.001 & 0.736 & -0.001 & 0.871 & -0.002 & 0.548 & -0.006 & 0.124 & 0.004 & 0.482 \\
\hline$\alpha_{3 \_} D_{m} l a g$ & 0.001 & 0.521 & -0.004 & 0.057 & 0.003 & 0.388 & 0.003 & 0.320 & -0.006 & 0.129 & 0.002 & 0.651 \\
\hline$\alpha_{4-} D_{m} l a g$ & -0.004 & 0.057 & -0.004 & 0.081 & 0.000 & 0.993 & -0.002 & 0.581 & -0.005 & 0.185 & 0.003 & 0.519 \\
\hline$\alpha_{5} D_{m} l a g$ & -0.002 & 0.116 & -0.001 & 0.403 & -0.001 & 0.688 & -0.003 & 0.216 & -0.004 & 0.231 & 0.000 & 0.934 \\
\hline$\beta_{0} D_{m} l a g$ & 0.005 & 0.804 & -0.009 & 0.269 & 0.005 & 0.817 & -0.064 & 0.005 & 0.101 & 0.078 & 0.037 & 0.548 \\
\hline$\beta_{1-} D_{m} l a g$ & -0.022 & 0.108 & -0.016 & 0.023 & -0.005 & 0.719 & 0.012 & 0.492 & -0.079 & 0.133 & 0.067 & 0.229 \\
\hline$\beta_{2} D_{m} l a g$ & -0.016 & 0.472 & 0.009 & 0.200 & -0.006 & 0.787 & 0.004 & 0.798 & 0.171 & 0.155 & 0.168 & 0.166 \\
\hline$\beta_{3-} D_{m} l a g$ & 0.044 & 0.004 & 0.006 & 0.426 & -0.038 & 0.028 & 0.025 & 0.128 & 0.069 & 0.147 & 0.043 & 0.385 \\
\hline$\beta_{4-} D_{m} l a g$ & -0.073 & 0.000 & 0.003 & 0.703 & -0.070 & 0.000 & 0.019 & 0.188 & 0.001 & 0.966 & -0.018 & 0.591 \\
\hline$\beta_{5} D_{m} l a g$ & 0.111 & 0.000 & 0.006 & 0.588 & -0.105 & 0.000 & -0.018 & 0.268 & 0.014 & 0.638 & -0.004 & 0.913 \\
\hline$D_{m} \operatorname{lag}, X$ & 0.106 & 0.000 & 0.018 & 0.580 & -0.088 & 0.038 & -0.057 & 0.408 & -0.027 & 0.767 & -0.030 & 0.789 \\
\hline$\gamma_{1 \_} D_{m} l a g$ & -0.015 & 0.023 & -0.006 & 0.329 & -0.009 & 0.314 & -0.013 & 0.090 & -0.009 & 0.261 & -0.003 & 0.758 \\
\hline$\gamma_{2-} D_{m} l a g$ & -0.008 & 0.206 & -0.005 & 0.360 & -0.003 & 0.746 & 0.007 & 0.342 & -0.010 & 0.219 & 0.003 & 0.775 \\
\hline$\gamma_{3-} D_{m} l a g$ & -0.006 & 0.392 & -0.001 & 0.810 & -0.004 & 0.634 & -0.003 & 0.606 & -0.011 & 0.162 & 0.007 & 0.474 \\
\hline$\gamma_{4-} D_{m} l a g$ & -0.004 & 0.522 & -0.009 & 0.117 & 0.005 & 0.523 & -0.003 & 0.660 & -0.021 & 0.005 & 0.018 & 0.068 \\
\hline$\gamma_{5-} D_{m} l a g$ & 0.006 & 0.327 & 0.004 & 0.379 & -0.001 & 0.847 & 0.000 & 0.985 & -0.012 & 0.065 & 0.012 & 0.170 \\
\hline$\delta_{1} \_D_{m} l a g$ & 0.006 & 0.004 & 0.002 & 0.320 & -0.003 & 0.249 & -0.002 & 0.490 & 0.004 & 0.333 & 0.001 & 0.796 \\
\hline$\delta_{2-} D_{m} l a g$ & 0.000 & 0.932 & 0.002 & 0.334 & 0.002 & 0.519 & 0.002 & 0.520 & -0.005 & 0.186 & 0.003 & 0.586 \\
\hline$\delta_{3-} D_{m} l a g$ & 0.002 & 0.362 & 0.003 & 0.195 & 0.001 & 0.740 & 0.002 & 0.547 & 0.010 & 0.007 & 0.008 & 0.092 \\
\hline$\delta_{4-} D_{m} l a g$ & 0.004 & 0.125 & -0.003 & 0.219 & -0.001 & 0.751 & -0.003 & 0.405 & 0.007 & 0.053 & 0.004 & 0.352 \\
\hline$\delta_{5-} D_{m} l a g$ & -0.001 & 0.464 & 0.001 & 0.597 & 0.000 & 0.914 & 0.007 & 0.023 & -0.005 & 0.213 & -0.002 & 0.631 \\
\hline$A \_D_{m} l a g$ & -0.006 & 0.338 & -0.013 & 0.055 & 0.007 & 0.454 & -0.005 & 0.631 & -0.025 & 0.032 & 0.020 & 0.204 \\
\hline$B \_D_{m} l a g$ & 0.049 & 0.016 & -0.001 & 0.953 & -0.047 & 0.111 & -0.021 & 0.552 & 0.277 & 0.108 & 0.256 & 0.147 \\
\hline$\Gamma_{-} D_{m} l a g$ & -0.026 & 0.110 & -0.017 & 0.285 & -0.009 & 0.689 & -0.012 & 0.565 & -0.064 & 0.006 & 0.052 & 0.091 \\
\hline$\Delta_{-} D_{m} l a g$ & 0.009 & 0.007 & 0.005 & 0.163 & -0.004 & 0.442 & 0.006 & 0.312 & 0.011 & 0.097 & 0.005 & 0.558 \\
\hline
\end{tabular}




\section{Table 4.13: P-Value for Tests of Model Parameters (WLS - Dummy Variable of One-day Lagged Market Return)}

The table is based on the model defined at (4.1) using 60 days of trade data by WLS interacted with a dummy variable of one-day lagged market return. The tests for individual parameters and sums are computed as described in Section 2.3.3. The cell entries show the estimated parameters interacted with the corresponding dummy variable and their probabilities (p-val.), respectively. Differences are calculated by absolute value of model parameters between after and before both events. $D_{\text {mlag }, t}$ is the dummy variable denoted 1 if the market return on that day is positive or 0 if it is non-positive. Bold format denotes significance at the $1 \%$ level.

\begin{tabular}{|c|c|c|c|c|c|c|c|c|c|c|c|c|}
\hline & \multicolumn{6}{|c|}{ Addition } & \multicolumn{6}{|c|}{ Deletion } \\
\hline & \multicolumn{2}{|c|}{ Before } & \multicolumn{2}{|c|}{ After } & \multicolumn{2}{|c|}{ Difference } & \multicolumn{2}{|c|}{ Before } & \multicolumn{2}{|c|}{ After } & \multicolumn{2}{|c|}{ Difference } \\
\hline & Coef. & p-val. & Coef. & p-val. & Coef. & p-val. & Coef. & p-val. & Coef. & p-val. & Coef. & p-val \\
\hline$D_{m} \operatorname{lag}, R$ & -0.004 & 0.025 & -0.002 & 0.515 & -0.003 & 0.377 & -0.008 & 0.263 & -0.007 & 0.399 & -0.001 & 0.961 \\
\hline$\alpha_{1 \_} D_{m} l a g$ & 0.000 & 0.869 & -0.001 & 0.766 & 0.000 & 0.964 & -0.008 & 0.038 & -0.006 & 0.159 & -0.002 & 0.756 \\
\hline$\alpha_{2 \_} D_{m} l a g$ & 0.001 & 0.714 & -0.001 & 0.608 & 0.000 & 0.966 & -0.006 & 0.105 & 0.004 & 0.546 & -0.002 & 0.727 \\
\hline$\alpha_{3-} D_{m} l a g$ & -0.005 & 0.116 & -0.008 & 0.002 & 0.003 & 0.494 & -0.003 & 0.478 & -0.010 & 0.012 & 0.007 & 0.206 \\
\hline$\alpha_{4-} D_{m} l a g$ & 0.000 & 0.939 & -0.003 & 0.217 & 0.003 & 0.427 & -0.004 & 0.277 & 0.010 & 0.016 & 0.007 & 0.221 \\
\hline$\alpha_{5-} D_{m} l a g$ & -0.004 & 0.056 & -0.001 & 0.584 & -0.002 & 0.432 & -0.001 & 0.696 & -0.004 & 0.321 & 0.003 & 0.598 \\
\hline$\beta_{0} D_{m} l a g$ & -0.008 & 0.614 & -0.004 & & -0.004 & & -0.118 & 0.000 & -0.002 & 0.989 & -0.116 & 0.401 \\
\hline$\beta_{1-} D_{m} l a g$ & -0.080 & 0.000 & 0.004 & 0.610 & -0.076 & 0.000 & 0.031 & 0.131 & -0.035 & 0.444 & 0.003 & 0.949 \\
\hline$\beta_{2-} D_{m} l a g$ & -0.005 & 0.576 & 0.051 & 0.000 & 0.046 & 0.000 & 0.024 & 0.125 & -0.036 & 0.262 & 0.012 & 0.735 \\
\hline$\beta_{3-} D_{m} l a g$ & 0.001 & 0.952 & 0.008 & 0.426 & 0.007 & 0.659 & -0.005 & 0.824 & 0.010 & 0.781 & 0.005 & 0.896 \\
\hline$\beta_{4-} D_{m} l a g$ & 0.010 & 0.397 & -0.004 & 0.728 & -0.006 & 0.719 & 0.053 & 0.021 & -0.040 & 0.460 & -0.013 & 0.821 \\
\hline$\beta_{5-} D_{m} l a g$ & -0.004 & 0.743 & 0.008 & 0.467 & 0.004 & 0.801 & 0.010 & 0.548 & -0.045 & 0.343 & 0.035 & 0.481 \\
\hline$D_{m} \operatorname{lag}, X$ & 0.030 & 0.015 & -0.008 & 0.498 & -0.022 & 0.202 & 0.002 & 0.932 & -0.030 & 0.212 & 0.028 & 0.404 \\
\hline$\gamma_{1} D_{m} l a g$ & -0.011 & 0.388 & 0.002 & 0.854 & -0.009 & 0.610 & -0.019 & 0.088 & -0.004 & 0.848 & -0.015 & 0.483 \\
\hline$\gamma_{2-} D_{m} l a g$ & -0.019 & 0.092 & 0.007 & 0.473 & -0.012 & & -0.003 & & 0.015 & 0.313 & 0.012 & 0.484 \\
\hline$\gamma_{3}{ }_{2} D_{m} l a g$ & 0.000 & 0.976 & -0.016 & 0.231 & 0.016 & 0.435 & 0.013 & 0.128 & -0.016 & 0.224 & 0.003 & 0.834 \\
\hline$\gamma_{4} D_{m} l a g$ & -0.007 & 0.511 & -0.004 & 0.779 & -0.002 & 0.891 & 0.012 & 0.144 & 0.002 & 0.897 & -0.010 & 0.491 \\
\hline$\gamma_{5-} D_{m} l a g$ & 0.011 & 0.430 & 0.009 & 0.283 & -0.002 & 0.893 & 0.017 & 0.022 & -0.013 & 0.275 & -0.004 & 0.773 \\
\hline$\delta_{1 \_} D_{m} l a g$ & 0.007 & 0.003 & 0.006 & 0.021 & -0.001 & 0.753 & 0.009 & 0.025 & 0.011 & 0.071 & 0.002 & 0.788 \\
\hline$\delta_{2-} D_{m} l a g$ & -0.001 & 0.662 & 0.001 & 0.792 & -0.001 & 0.872 & 0.003 & 0.468 & -0.008 & 0.061 & 0.005 & 0.411 \\
\hline$\delta_{3} D_{m} l a g$ & 0.003 & 0.084 & 0.004 & 0.078 & 0.000 & 0.910 & 0.002 & 0.499 & -0.007 & 0.240 & 0.005 & 0.469 \\
\hline$\delta_{4-} D_{m} l a g$ & 0.008 & 0.200 & -0.004 & 0.055 & -0.004 & 0.580 & -0.002 & 0.555 & 0.008 & 0.108 & 0.006 & 0.325 \\
\hline$\delta_{5-} D_{m} l a g g$ & -0.010 & 0.304 & 0.007 & 0.001 & -0.003 & 0.795 & 0.004 & 0.169 & 0.002 & 0.698 & -0.003 & 0.617 \\
\hline$A_{-} D_{m} l a g$ & -0.007 & 0.375 & -0.013 & 0.037 & 0.006 & 0.565 & -0.022 & 0.032 & -0.007 & 0.600 & -0.015 & 0.361 \\
\hline$B_{-} D_{m} l a g$ & -0.086 & 0.004 & 0.063 & 0.035 & -0.023 & 0.589 & -0.004 & 0.914 & -0.147 & 0.354 & 0.143 & 0.383 \\
\hline$\Gamma_{-} D_{m} \operatorname{lag}$ & -0.026 & 0.403 & -0.003 & 0.924 & -0.023 & 0.586 & 0.021 & 0.367 & -0.017 & 0.664 & -0.004 & 0.921 \\
\hline$\Delta_{\_} D_{m} l a g$ & 0.008 & 0.216 & 0.013 & 0.002 & 0.006 & 0.454 & 0.017 & 0.012 & 0.006 & 0.464 & -0.011 & 0.296 \\
\hline
\end{tabular}


Table 4.14: P-Value for Tests of Model Parameters (GARCH - Dummy Variable of One-day Lagged Market Return)

The table is based on the model defined at (4.1) using 60 days of trade data by GARCH $(1,1)$ interacted with a dummy variable of one-day lagged market return. The tests for individual parameters and sums are computed as described in Section 2.3.3. The cell entries show the estimated parameters interacted with the corresponding dummy variable and their probabilities (p-val.), respectively. Differences are calculated by absolute value of model parameters between after and before both events. $D_{m l a g, t}$ is the dummy variable denoted 1 if the market return on that day is positive or 0 if it is non-positive. Bold format denotes significance at the $1 \%$ level.

\begin{tabular}{|c|c|c|c|c|c|c|c|c|c|c|c|c|}
\hline & \multicolumn{6}{|c|}{ Addition } & \multicolumn{6}{|c|}{ Deletion } \\
\hline & \multicolumn{2}{|c|}{ Before } & \multicolumn{2}{|c|}{ After } & \multicolumn{2}{|c|}{ Difference } & \multicolumn{2}{|c|}{ Before } & \multicolumn{2}{|c|}{ After } & \multicolumn{2}{|c|}{ Difference } \\
\hline & Coef. & p-val. & Coef. & p-val. & Coef. & p-val. & Coef. & p-val. & Coef. & p-val. & Coef. & p-val. \\
\hline$D_{m} l a g, R$ & -0.011 & 0.940 & 0.019 & 0.981 & 0.008 & 0.992 & -0.029 & 0.574 & 0.045 & 0.843 & 0.016 & 0.944 \\
\hline$\alpha_{1-} D_{m} l a g$ & 0.002 & 0.615 & -0.004 & 0.908 & 0.002 & 0.949 & -0.004 & 0.266 & 0.002 & 0.950 & -0.003 & 0.919 \\
\hline$\alpha_{2-} D_{m} l a g$ & -0.002 & 0.565 & 0.006 & 0.819 & 0.005 & 0.866 & 0.000 & 0.988 & 0.009 & 0.733 & 0.008 & 0.738 \\
\hline$\alpha_{3} D_{m} l a g$ & 0.000 & 0.997 & -0.002 & 0.936 & 0.002 & 0.965 & 0.001 & 0.750 & -0.005 & 0.859 & 0.004 & 0.891 \\
\hline$\alpha_{4-} D_{m} l a g$ & -0.003 & 0.956 & -0.002 & 0.834 & -0.001 & 0.988 & 0.002 & 0.605 & 0.002 & 0.937 & 0.001 & 0.986 \\
\hline$\alpha_{5} D_{m} l a g$ & -0.001 & 0.955 & -0.001 & 0.915 & -0.001 & 0.984 & 0.000 & 0.886 & 0.000 & 0.999 & 0.000 & 0.975 \\
\hline$\beta_{0-} D_{m} l a g$ & 0.009 & 0.982 & -0.010 & 0.802 & 0.001 & 0.998 & -0.048 & 0.002 & 0.112 & 0.284 & 0.065 & 0.542 \\
\hline$\beta_{1-} D_{m} l a g$ & 0.021 & 0.961 & -0.004 & 0.925 & -0.017 & 0.968 & -0.044 & 0.049 & -0.033 & 0.787 & -0.011 & 0.929 \\
\hline$\beta_{2} D_{m} l a g$ & -0.023 & 0.921 & -0.008 & 0.767 & -0.014 & 0.950 & -0.001 & 0.972 & -0.001 & 0.993 & 0.000 & 0.999 \\
\hline$\beta_{3} D_{m} l a g$ & -0.006 & 0.984 & -0.003 & 0.935 & -0.003 & 0.992 & -0.007 & 0.713 & 0.041 & 0.744 & 0.034 & 0.790 \\
\hline$\beta_{4-} D_{m} l a g$ & -0.054 & 0.889 & -0.009 & 0.799 & -0.045 & 0.908 & -0.004 & 0.819 & -0.038 & 0.702 & 0.034 & 0.734 \\
\hline$\beta_{5-} D_{m} l a g$ & 0.136 & 0.885 & -0.018 & 0.664 & -0.118 & 0.901 & -0.024 & 0.160 & 0.049 & 0.651 & 0.025 & 0.819 \\
\hline$D_{m} \operatorname{lag}, X$ & 0.100 & 0.000 & 0.031 & 0.469 & -0.069 & 0.149 & -0.094 & 0.383 & -0.097 & 0.499 & 0.004 & 0.984 \\
\hline$\gamma_{1} D_{m} l a g$ & -0.030 & 0.000 & 0.000 & 0.976 & -0.030 & 0.001 & -0.001 & 0.946 & 0.000 & 0.993 & -0.001 & 0.964 \\
\hline$\gamma_{2} D_{m} l a g$ & -0.010 & 0.047 & 0.006 & 0.447 & -0.004 & 0.677 & 0.019 & 0.492 & -0.020 & 0.278 & 0.001 & 0.972 \\
\hline$\gamma_{3} D_{m} l a g$ & -0.010 & 0.038 & 0.000 & 0.996 & -0.010 & 0.326 & 0.008 & 0.621 & -0.011 & 0.541 & 0.003 & 0.900 \\
\hline$\gamma_{4-} D_{m} l a g$ & -0.006 & 0.243 & -0.006 & 0.505 & 0.000 & 0.987 & 0.016 & 0.478 & -0.024 & 0.152 & 0.009 & 0.761 \\
\hline$\gamma_{5} D_{m} l a g$ & 0.014 & 0.005 & -0.010 & 0.203 & -0.004 & 0.677 & 0.006 & 0.606 & -0.025 & 0.093 & 0.019 & 0.328 \\
\hline$\delta_{1 \_} D_{m} l a g$ & 0.004 & 0.641 & 0.001 & 0.904 & -0.003 & 0.778 & 0.005 & 0.617 & -0.004 & 0.890 & -0.001 & 0.969 \\
\hline$\delta_{2-} D_{m} l a g$ & 0.001 & 0.892 & 0.005 & 0.356 & 0.004 & 0.657 & -0.001 & 0.836 & 0.002 & 0.946 & 0.001 & 0.984 \\
\hline$\delta_{3-} D_{m} l a g$ & 0.003 & 0.606 & 0.005 & 0.523 & 0.002 & 0.865 & 0.005 & 0.745 & 0.015 & 0.608 & 0.010 & 0.755 \\
\hline$\delta_{4-} D_{m} l a g$ & 0.002 & 0.785 & -0.003 & 0.565 & 0.001 & 0.951 & -0.002 & 0.773 & 0.002 & 0.931 & 0.000 & 0.986 \\
\hline$\delta_{5-} D_{m} l a g$ & -0.004 & 0.658 & 0.003 & 0.656 & -0.001 & 0.923 & -0.002 & 0.845 & -0.002 & 0.919 & 0.001 & 0.975 \\
\hline$A_{-} D_{m} l a g$ & -0.004 & 0.965 & -0.002 & 0.981 & -0.002 & 0.988 & -0.001 & 0.924 & 0.007 & 0.941 & 0.006 & 0.949 \\
\hline$B \_D_{m} l a g$ & 0.084 & 0.909 & -0.053 & 0.527 & -0.031 & 0.967 & -0.128 & 0.002 & 0.131 & 0.681 & 0.003 & 0.994 \\
\hline$\Gamma_{-} D_{m} l a g$ & -0.042 & 0.002 & -0.010 & 0.656 & -0.033 & 0.199 & 0.047 & 0.380 & -0.080 & 0.111 & 0.033 & 0.657 \\
\hline$\Delta_{-} D_{m} l a g$ & 0.006 & 0.453 & 0.011 & 0.253 & 0.004 & 0.739 & 0.005 & 0.722 & 0.013 & 0.770 & 0.008 & 0.859 \\
\hline
\end{tabular}




\section{Table 4.15: P-Value for Tests of Model Parameters - Crisis (Addition)}

Based on the models of OLS, WLS, VAR, WVAR, GARCH $(1,1)$ and BEKK $(1,1)$ using 60 days of trade data, the tests for sums of $A$ and $\Delta$ measuring speed of price adjustment for the addition events are computed as described in Section 2.3.3 for crisis and noncrisis periods. The cell entries of the first panel show the number of observations in crisis and non-crisis periods. The cell entries of other panels show the estimated parameters and probabilities (p-val.), respectively. Differences for crisis and non-crisis periods are calculated by absolute value of model parameters between after and before for the event (After minus Before). Differences between crisis and non-crisis periods before and after the events are calculated by absolute value of model parameters between crisis and noncrisis periods (Crisis minus Non-crisis) before and after the events. The crisis period is between December 2007 and June 2009. The event is defined as in the crisis period if the whole estimation period of the event ( 60 days before and after the effective date) is in the crisis period. Crisis $=1$ indicates crisis period and crisis $=0$ indicates non-crisis period. Bold format denotes significance at the $1 \%$ level.

\begin{tabular}{|c|c|c|c|c|c|c|c|c|c|c|c|c|}
\hline & \multicolumn{12}{|c|}{ Addition } \\
\hline & Before & After & Diff. & p-val. & Before & After & Diff. & p-val. & Diff. in Before & p-val. & Diff. in After & p-val. \\
\hline \multicolumn{13}{|c|}{ OLS } \\
\hline$A$ & -0.445 & -0.445 & -0.001 & 0.897 & -0.533 & -0.520 & -0.013 & 0.641 & 0.087 & 0.000 & 0.075 & 0.000 \\
\hline$\Delta$ & 0.333 & 0.324 & -0.009 & 0.003 & 0.327 & 0.287 & $\begin{array}{r}-0.040 \\
\text { WLS }\end{array}$ & 0.004 & -0.006 & 0.520 & -0.038 & 0.000 \\
\hline$A$ & -0.238 & -0.226 & -0.013 & 0.009 & -0.267 & -0.363 & 0.096 & 0.000 & 0.029 & 0.044 & 0.137 & 0.000 \\
\hline$\Delta$ & 0.317 & 0.305 & -0.012 & 0.000 & 0.288 & 0.263 & $\begin{array}{r}-0.025 \\
\text { VAR }\end{array}$ & 0.134 & -0.030 & 0.010 & -0.042 & 0.001 \\
\hline$A$ & -0.410 & -0.403 & -0.007 & 0.006 & -0.466 & -0.485 & 0.019 & 0.126 & 0.056 & 0.000 & 0.082 & 0.000 \\
\hline$\Delta$ & \multicolumn{11}{|c|}{ WVAR } & 0.000 \\
\hline$A$ & -0.223 & -0.211 & -0.012 & 0.000 & -0.238 & -0.269 & 0.031 & 0.006 & 0.015 & 0.037 & 0.058 & 0.000 \\
\hline \multicolumn{12}{|c|}{ GARCH } & 0.000 \\
\hline$A$ & -0.473 & -0.474 & 0.000 & 0.943 & -0.513 & -0.556 & 0.043 & 0.458 & 0.040 & 0.000 & 0.083 & 0.150 \\
\hline \multicolumn{12}{|c|}{ BEKK } & 0.000 \\
\hline$A$ & -0.436 & -0.421 & -0.015 & 0.000 & -0.512 & -0.537 & 0.024 & 0.153 & 0.076 & 0.000 & 0.115 & 0.000 \\
\hline$\Delta$ & 0.373 & 0.359 & -0.014 & 0.000 & 0.396 & 0.302 & -0.093 & 0.000 & 0.023 & 0.000 & -0.056 & 0.000 \\
\hline
\end{tabular}




\section{Table 4.16: P-Value for Tests of Model Parameters - Crisis (Deletion)}

Based on the models of OLS, WLS, VAR, WVAR, GARCH $(1,1)$ and BEKK $(1,1)$ using 60 days of trade data, the tests for sums of $A$ and $\Delta$ measuring speed of price adjustment for the deletion events are computed as described in Section 2.3.3 for crisis and non-crisis periods. The cell entries of the first panel show the number of observations in crisis and non-crisis periods. The cell entries of other panels show the estimated parameters and probabilities (p-val.), respectively. Differences for crisis and non-crisis periods are calculated by absolute value of model parameters between after and before for the event (After minus Before). Differences between crisis and non-crisis periods before and after the events are calculated by absolute value of model parameters between crisis and noncrisis periods (Crisis minus Non-crisis) before and after the events. The crisis period is between December 2007 and June 2009. The event is defined as in the crisis period if the whole estimation period of the event (60 days before and after the effective date) is in the crisis period. Crisis $=1$ indicates crisis period and crisis $=0$ indicates non-crisis period. Bold format denotes significance at the $1 \%$ level.

\begin{tabular}{|c|c|c|c|c|c|c|c|c|c|c|c|c|}
\hline & \multicolumn{11}{|c|}{ Deletion } & \\
\hline & Before & After & Diff. & p-val. & Before & After & Diff. & p-val. & Diff. in Before & p-val. & Diff. in After & p-val. \\
\hline \multicolumn{13}{|c|}{ OLS } \\
\hline$A$ & -0.519 & -0.552 & 0.033 & 0.001 & -0.602 & -0.636 & 0.034 & 0.078 & 0.082 & 0.000 & 0.083 & 0.000 \\
\hline$\Delta$ & 0.342 & 0.345 & 0.003 & 0.545 & 0.311 & 0.324 & $\begin{array}{r}0.013 \\
\text { WLS }\end{array}$ & 0.218 & -0.031 & 0.000 & -0.022 & 0.018 \\
\hline$A$ & -0.251 & -0.267 & 0.016 & 0.059 & -0.291 & -0.279 & -0.012 & 0.440 & 0.040 & 0.001 & 0.012 & 0.327 \\
\hline$\Delta$ & 0.319 & 0.312 & -0.007 & 0.274 & 0.274 & 0.290 & $\begin{array}{r}0.017 \\
\text { VAP }\end{array}$ & 0.118 & -0.046 & 0.000 & -0.022 & 0.020 \\
\hline$A$ & -0.453 & -0.475 & 0.022 & 0.000 & -0.522 & -0.538 & 0.017 & 0.058 & 0.069 & 0.000 & 0.064 & 0.000 \\
\hline$\Delta$ & 0.331 & 0.338 & 0.007 & 0.055 & 0.291 & 0.311 & $\begin{array}{c}0.019 \\
\text { WVA }\end{array}$ & 0.001 & -0.039 & 0.000 & -0.027 & 0.000 \\
\hline$A$ & -0.229 & -0.238 & 0.010 & 0.038 & -0.243 & -0.256 & 0.013 & 0.099 & 0.014 & 0.018 & 0.018 & 0.012 \\
\hline$\Delta$ & 0.312 & 0.308 & -0.004 & 0.325 & 0.260 & 0.284 & $\begin{array}{l}0.024 \\
\text { GARC }\end{array}$ & $\begin{array}{l}0.000 \\
\end{array}$ & -0.051 & 0.000 & -0.024 & 0.000 \\
\hline$A$ & -0.550 & -0.573 & 0.023 & 0.017 & -0.616 & -0.634 & 0.018 & 0.647 & 0.066 & 0.000 & 0.062 & 0.098 \\
\hline$\Delta$ & 0.380 & 0.389 & 0.009 & 0.093 & 0.344 & 0.366 & $\begin{array}{r}0.022 \\
\text { BEK }\end{array}$ & 0.121 & -0.036 & 0.000 & -0.023 & 0.107 \\
\hline$A$ & -0.477 & -0.492 & 0.015 & 0.007 & -0.529 & -0.540 & 0.011 & 0.275 & 0.052 & 0.000 & 0.048 & 0.000 \\
\hline$\Delta$ & 0.359 & 0.363 & 0.004 & 0.325 & 0.330 & 0.324 & -0.006 & 0.402 & -0.029 & 0.000 & -0.038 & 0.000 \\
\hline
\end{tabular}




\section{Table 4.17: P-Value for Tests of Model Parameters Down and Up Markets (Addition)}

Based on the models of OLS, WLS, VAR, WVAR, GARCH $(1,1)$, and $\operatorname{BEKK}(1,1)$ using 60 days of trade data, the tests for sums of $A$ and $\Delta$ measuring speed of price adjustment for the addition events are computed as described in Section 2.3.3 for down and up markets. The cell entries show the estimated parameters and probabilities (p-val.), respectively. Differences for down and up markets are calculated by absolute value of model parameters between after and before for the event (After minus Before). Differences between down and up markets after the addition events are calculated by absolute value of model parameters after the event between down and up markets (Down minus Up). Transaction data before and after the event is divided into up (down) market group if the corresponding daily market return is positive (non-positive). Bold format denotes significance at the $1 \%$ level.

\begin{tabular}{|c|c|c|c|c|c|c|c|c|c|c|c|c|}
\hline & \multicolumn{12}{|c|}{ Addition } \\
\hline & \multicolumn{4}{|c|}{ Up Market } & \multicolumn{4}{|c|}{ Down Market } & \multicolumn{4}{|c|}{ Down - Up } \\
\hline & Before & After & Diff. & p-val. & Before & After & Diff. & p-val. & Diff. in Before & p-val. & Diff. in After & p-val. \\
\hline \multicolumn{13}{|c|}{ OLS } \\
\hline$A$ & -0.458 & -0.453 & -0.005 & 0.366 & -0.446 & -0.450 & 0.004 & 0.464 & -0.013 & 0.024 & -0.003 & 0.608 \\
\hline$\Delta$ & 0.333 & 0.328 & -0.005 & 0.162 & 0.330 & 0.324 & $\begin{array}{r}-0.005 \\
\text { WLS }\end{array}$ & 0.202 & -0.003 & 0.417 & -0.003 & 0.369 \\
\hline$A$ & -0.253 & -0.238 & -0.015 & 0.003 & -0.246 & -0.239 & -0.006 & 0.229 & -0.007 & 0.130 & 0.001 & 0.788 \\
\hline$\Delta$ & 0.317 & 0.308 & -0.009 & 0.030 & 0.316 & 0.307 & $\begin{array}{r}-0.009 \\
\text { VAR }\end{array}$ & 0.087 & -0.002 & 0.765 & -0.002 & 0.729 \\
\hline$A$ & -0.427 & -0.414 & -0.013 & 0.000 & -0.410 & -0.416 & 0.005 & 0.139 & -0.017 & 0.000 & 0.001 & 0.728 \\
\hline$\Delta$ & \multicolumn{12}{|c|}{ WVAR } \\
\hline$A$ & -0.240 & -0.220 & -0.020 & 0.000 & -0.228 & -0.227 & -0.001 & 0.739 & -0.012 & 0.000 & 0.007 & 0.023 \\
\hline$\Delta$ & 0.307 & 0.298 & -0.009 & 0.000 & 0.305 & 0.294 & -0.011 & 0.000 & -0.003 & 0.255 & -0.005 & 0.078 \\
\hline \multicolumn{13}{|c|}{ GARCH } \\
\hline$A$ & -0.485 & -0.482 & -0.003 & 0.555 & -0.472 & -0.472 & 0.000 & 0.982 & -0.013 & 0.114 & -0.010 & 0.061 \\
\hline$\Delta$ & 0.387 & 0.382 & -0.005 & 0.467 & 0.375 & 0.372 & $\begin{array}{c}-0.003 \\
\text { BEKK }\end{array}$ & 0.468 & -0.012 & 0.041 & -0.010 & 0.016 \\
\hline$A$ & -0.450 & -0.440 & -0.011 & 0.005 & -0.433 & -0.434 & 0.001 & 0.830 & -0.017 & 0.000 & -0.006 & 0.164 \\
\hline$\Delta$ & 0.365 & 0.357 & -0.007 & 0.006 & 0.358 & 0.345 & -0.013 & 0.000 & -0.006 & 0.016 & -0.013 & 0.000 \\
\hline
\end{tabular}




\section{Table 4.18: P-Value for Tests of Model Parameters Down and Up Markets (Deletion)}

Based on the models of OLS, WLS, VAR, WVAR, GARCH $(1,1)$, and BEKK $(1,1)$ using 60 days of trade data, the tests for sums of $A$ and $\Delta$ measuring speed of price adjustment for the deletion events are computed as described in Section 2.3.3 for down and up markets. The cell entries show the estimated parameters and probabilities (p-val.), respectively. Differences for down and up markets are calculated by absolute value of model parameters between after and before for the event (After minus Before). Differences between down and up markets after the addition events are calculated by absolute value of model parameters after the event between down and up markets (Down minus Up). Transaction data before and after the event is divided into up (down) market group if the corresponding daily market return is positive (non-positive). Bold format denotes significance at the $1 \%$ level.

\begin{tabular}{|c|c|c|c|c|c|c|c|c|c|c|c|c|}
\hline \multicolumn{13}{|c|}{ Deletion } \\
\hline & \multicolumn{4}{|c|}{ Up Market } & \multicolumn{4}{|c|}{ Down Market } & \multicolumn{4}{|c|}{ Down - Up } \\
\hline & Before & After & Diff. & p-val. & Before & After & Diff. & p-val. & Diff. in Before & p-val. & Diff. in After & p-val. \\
\hline \multicolumn{13}{|c|}{ OLS } \\
\hline$A$ & -0.550 & -0.584 & 0.034 & 0.002 & -0.540 & -0.578 & 0.038 & 0.000 & -0.010 & 0.318 & -0.006 & 0.607 \\
\hline$\Delta$ & 0.335 & 0.339 & 0.004 & 0.529 & 0.333 & 0.346 & $\begin{array}{r}0.012 \\
\text { WLS }\end{array}$ & 0.048 & -0.001 & 0.841 & 0.007 & 0.327 \\
\hline$A$ & -0.261 & -0.270 & 0.008 & 0.360 & -0.282 & -0.276 & -0.006 & 0.560 & 0.020 & 0.029 & 0.006 & 0.539 \\
\hline$\Delta$ & 0.310 & 0.310 & 0.000 & 0.993 & 0.303 & 0.311 & $\begin{array}{r}0.009 \\
\text { VAR }\end{array}$ & 0.269 & -0.007 & 0.334 & 0.001 & 0.893 \\
\hline$A$ & -0.476 & -0.510 & 0.034 & 0.000 & -0.477 & -0.501 & 0.024 & 0.000 & 0.001 & 0.918 & -0.009 & 0.176 \\
\hline$\Delta$ & 0.310 & 0.318 & 0.008 & 0.050 & 0.311 & 0.321 & $\begin{array}{c}0.011 \\
\text { WVA }\end{array}$ & 0.015 & 0.001 & 0.876 & 0.003 & 0.487 \\
\hline$A$ & -0.237 & -0.249 & 0.012 & 0.041 & -0.256 & -0.261 & 0.005 & 0.442 & 0.019 & 0.001 & 0.012 & 0.060 \\
\hline$\Delta$ & 0.289 & 0.296 & 0.007 & 0.115 & 0.282 & 0.292 & $\begin{array}{l}0.010 \\
\text { GARC }\end{array}$ & 0.039 & -0.006 & 0.123 & -0.004 & 0.421 \\
\hline$A$ & -0.566 & -0.591 & 0.025 & 0.013 & -0.559 & -0.591 & 0.032 & 0.007 & -0.007 & 0.373 & 0.000 & 0.985 \\
\hline$\Delta$ & 0.368 & 0.377 & 0.008 & 0.418 & 0.374 & 0.383 & $\begin{array}{r}0.009 \\
\text { BEK }\end{array}$ & 0.416 & 0.006 & 0.478 & 0.006 & 0.613 \\
\hline$A$ & -0.499 & -0.521 & 0.022 & 0.002 & -0.511 & -0.512 & 0.001 & 0.935 & 0.013 & 0.093 & -0.008 & 0.245 \\
\hline$\Delta$ & 0.339 & 0.340 & 0.001 & 0.849 & 0.343 & 0.347 & 0.004 & 0.451 & 0.004 & 0.413 & 0.007 & 0.138 \\
\hline
\end{tabular}




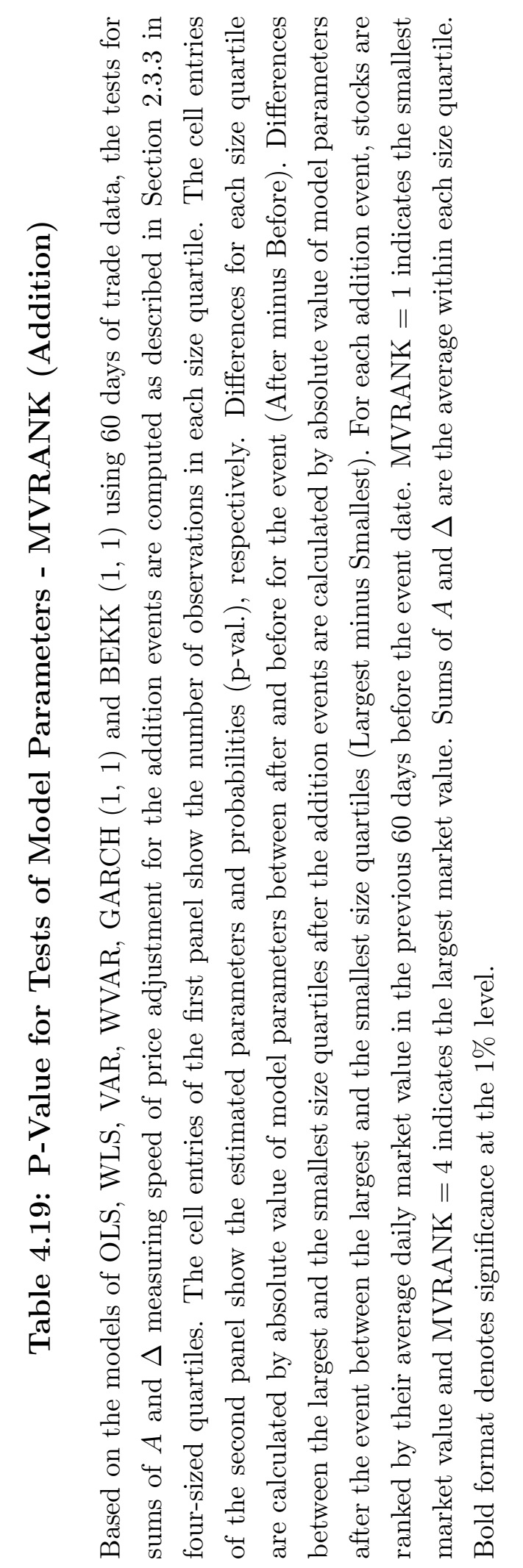

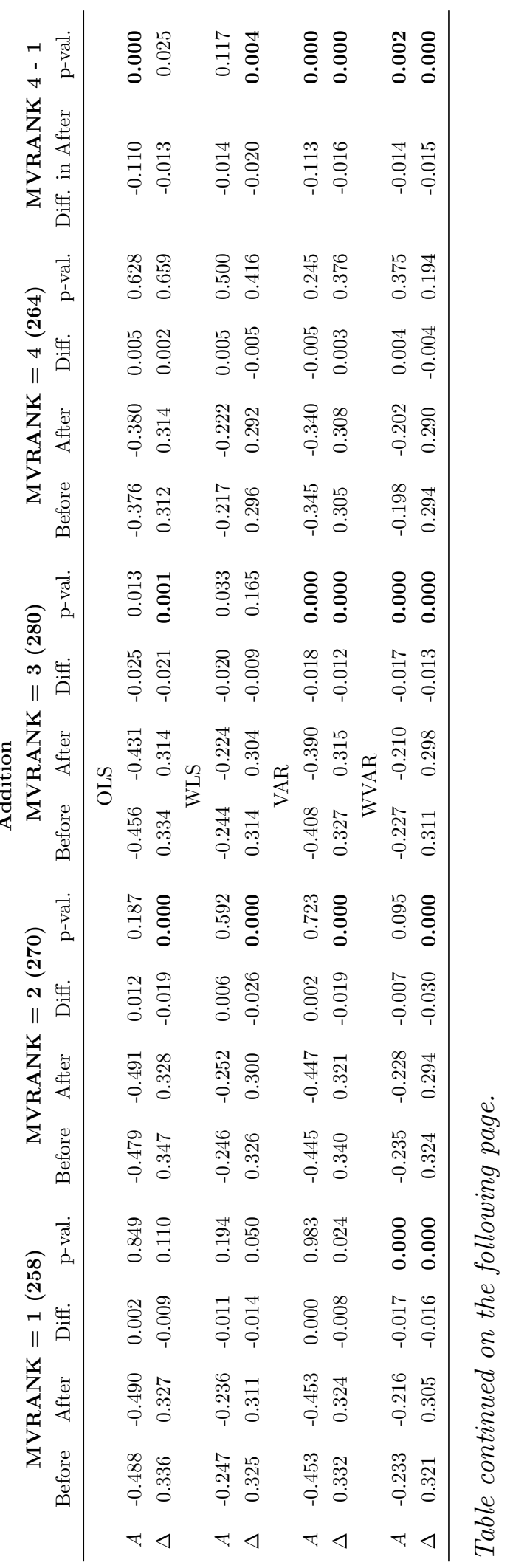




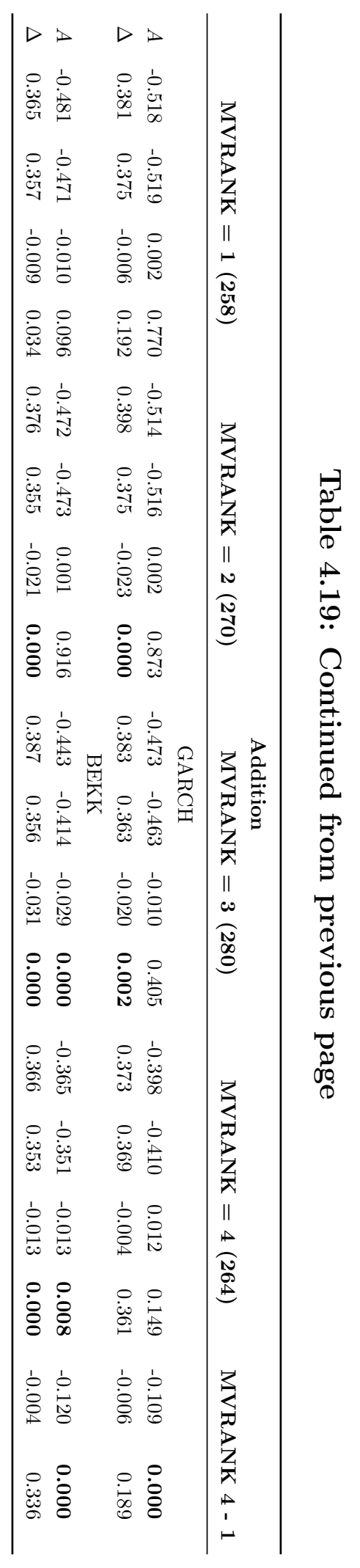




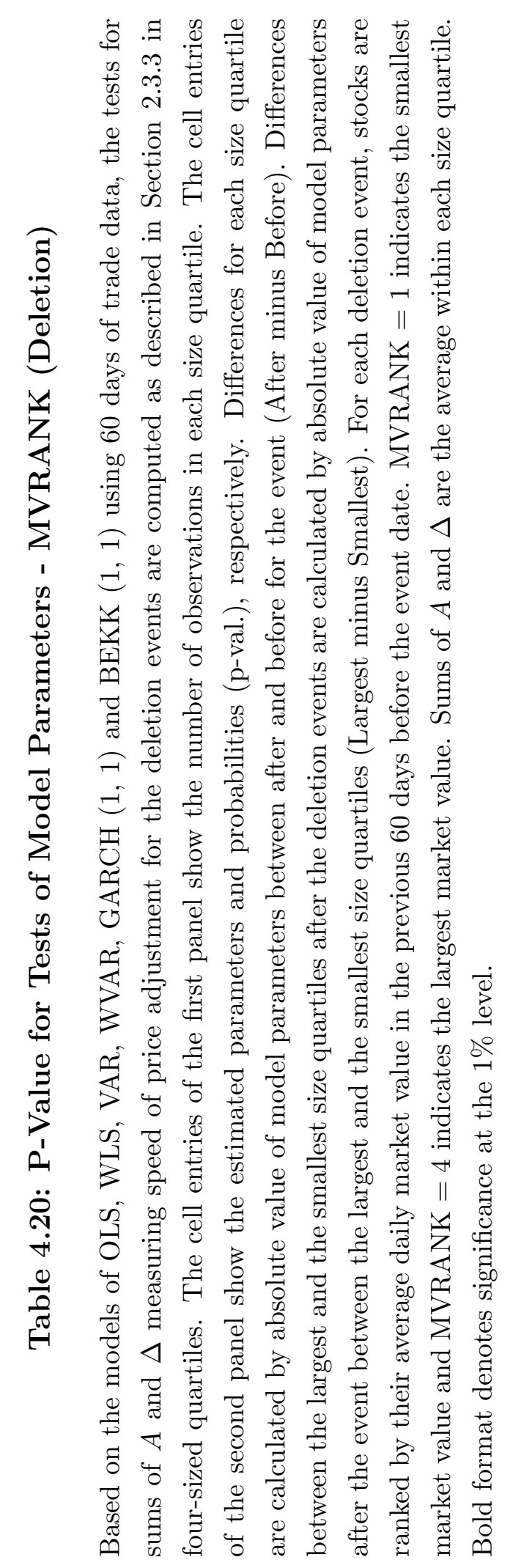

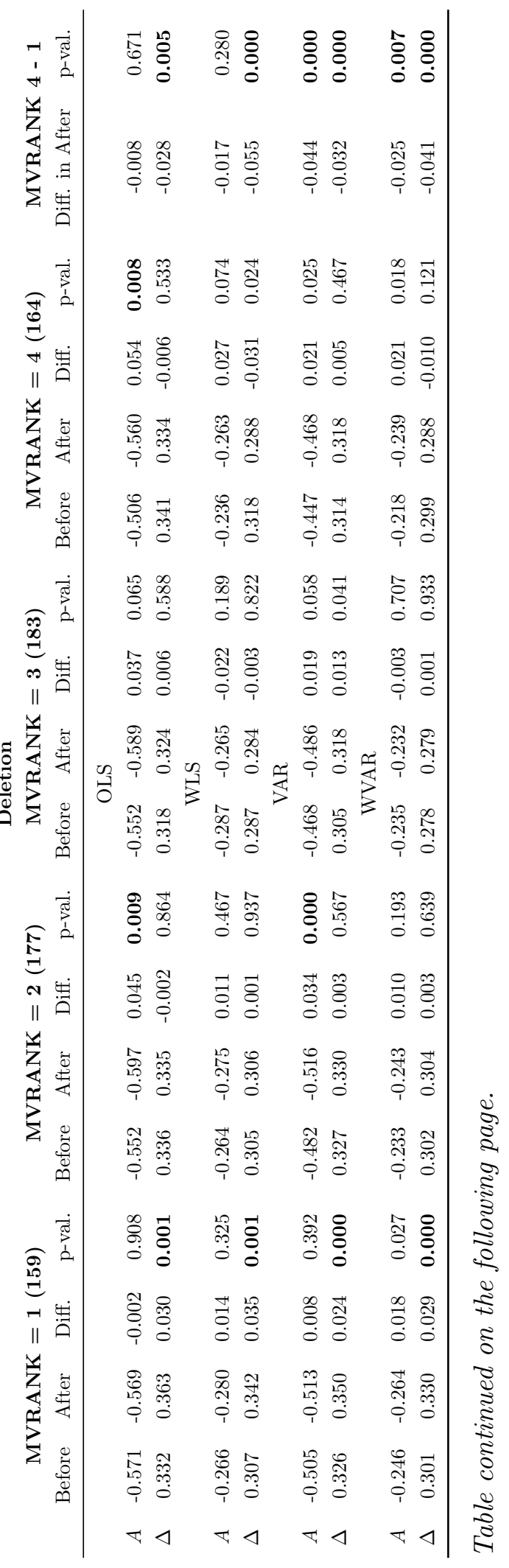




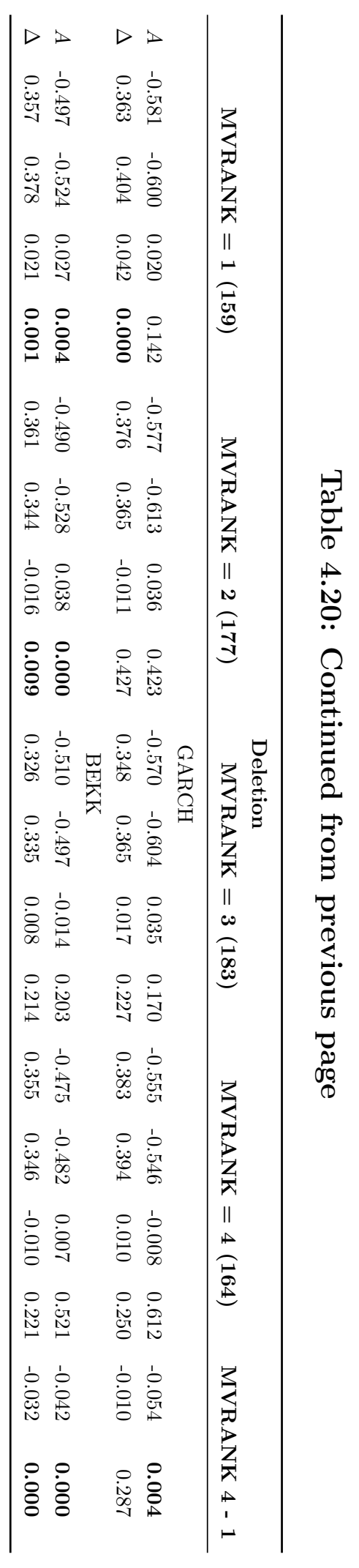




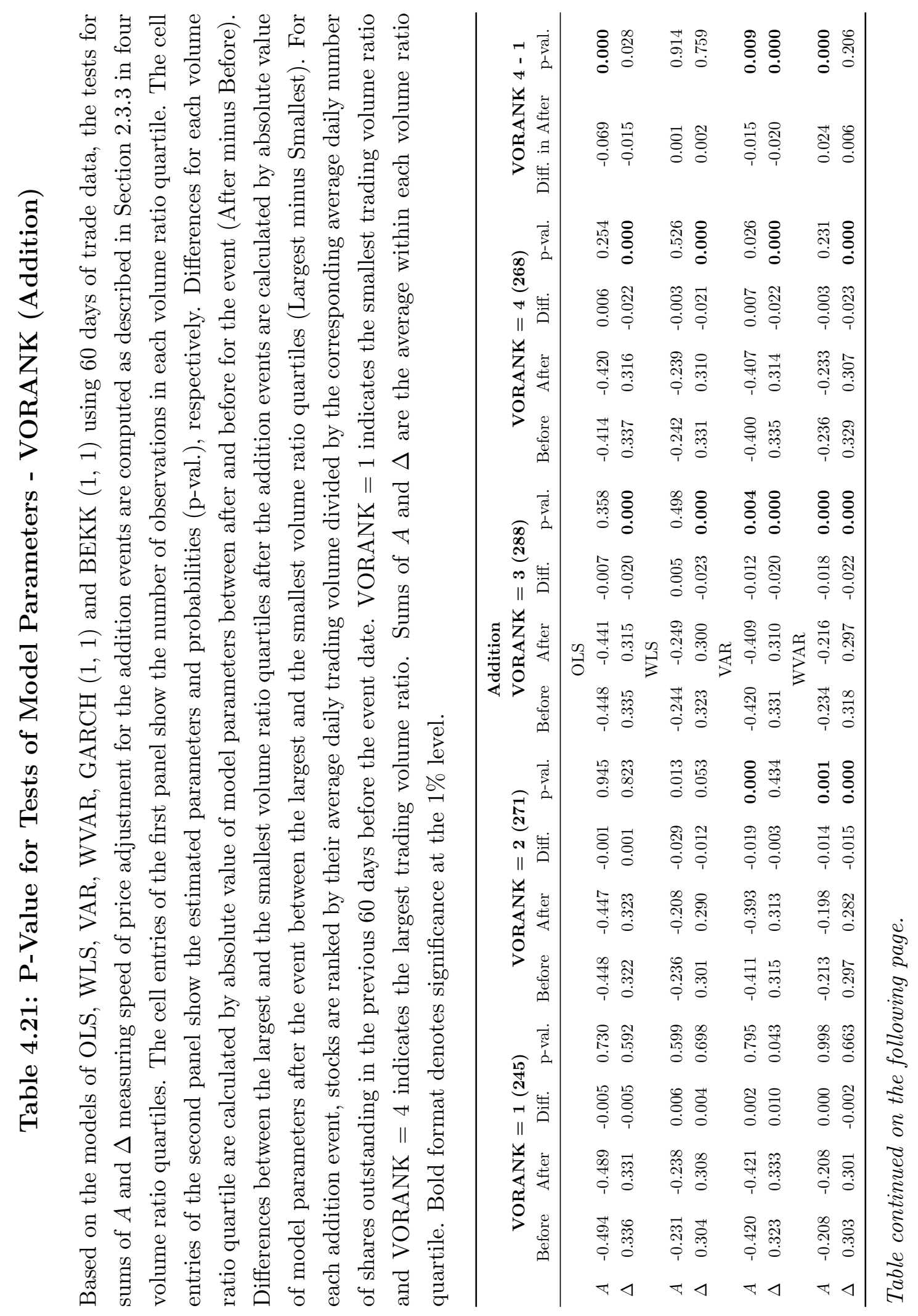




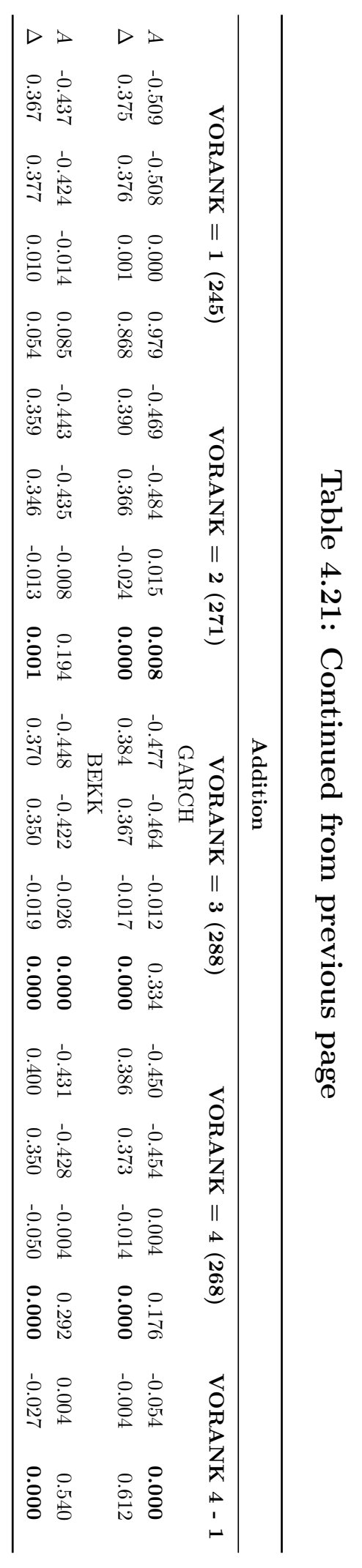




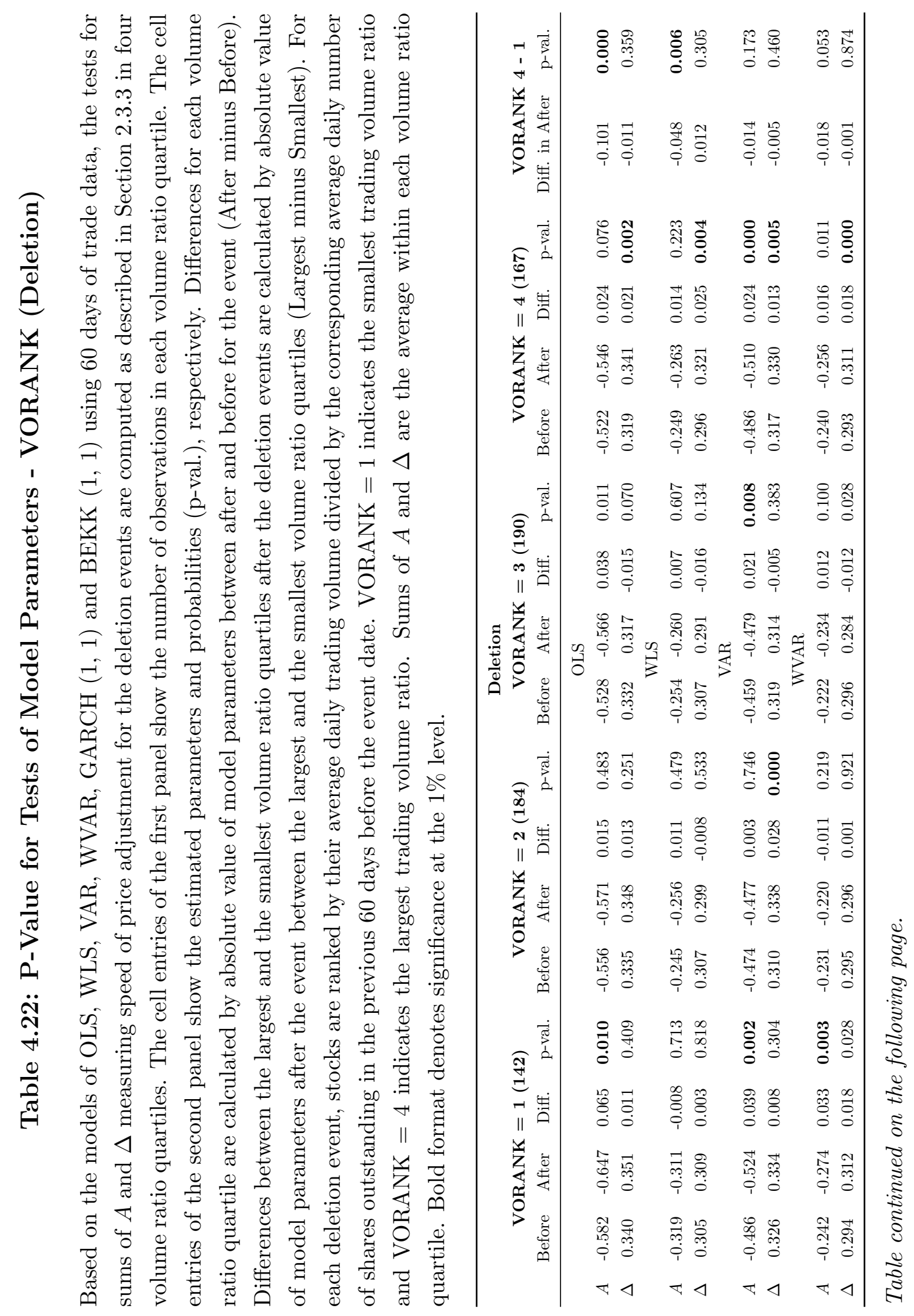




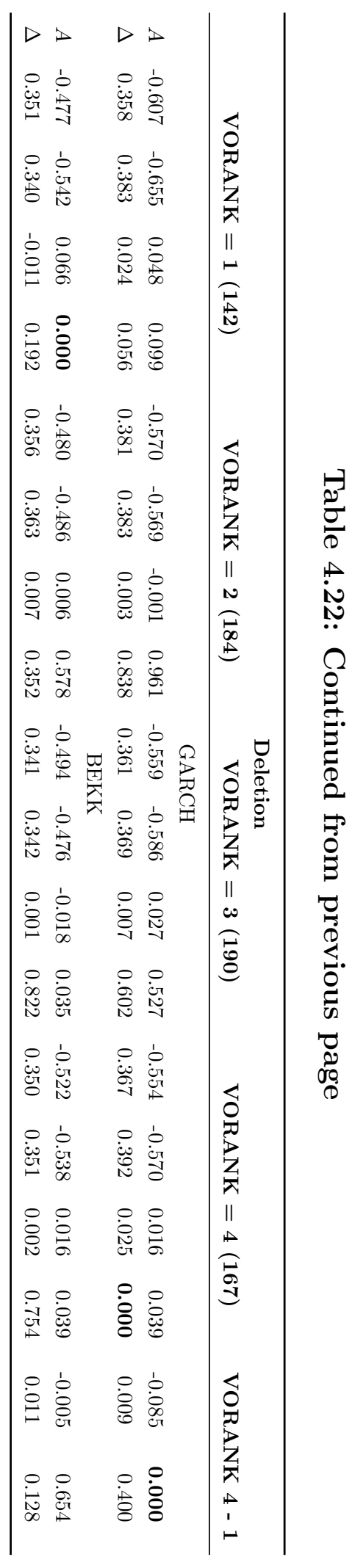




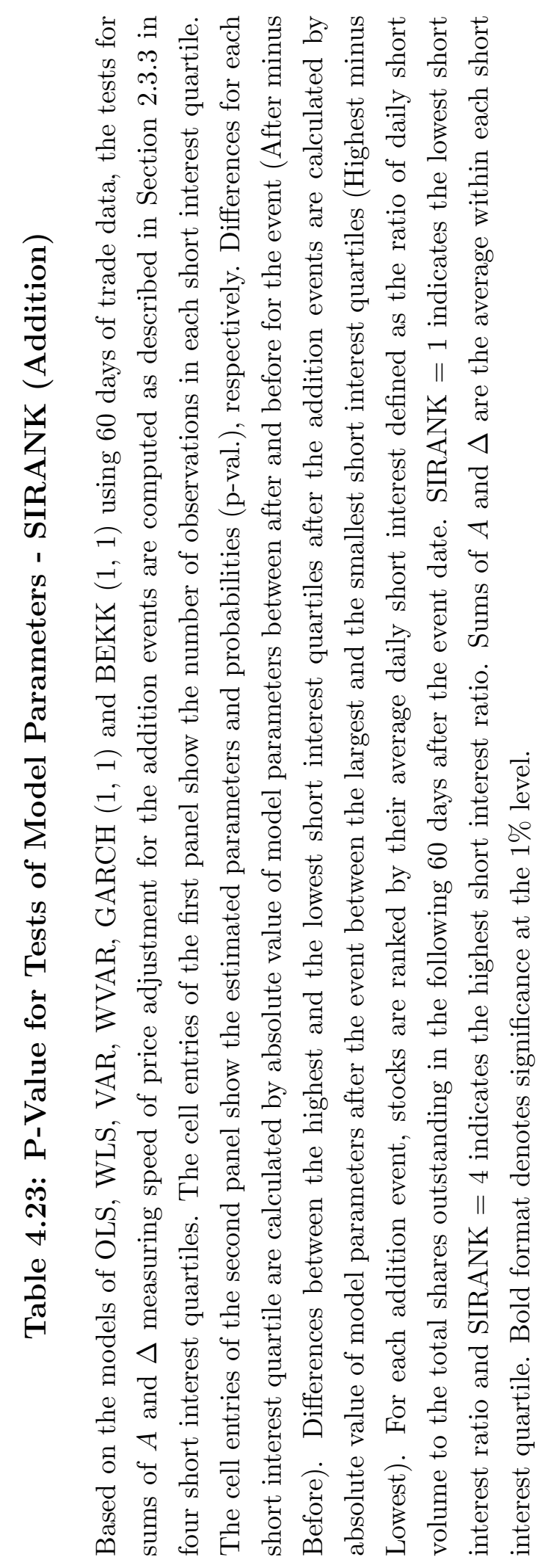

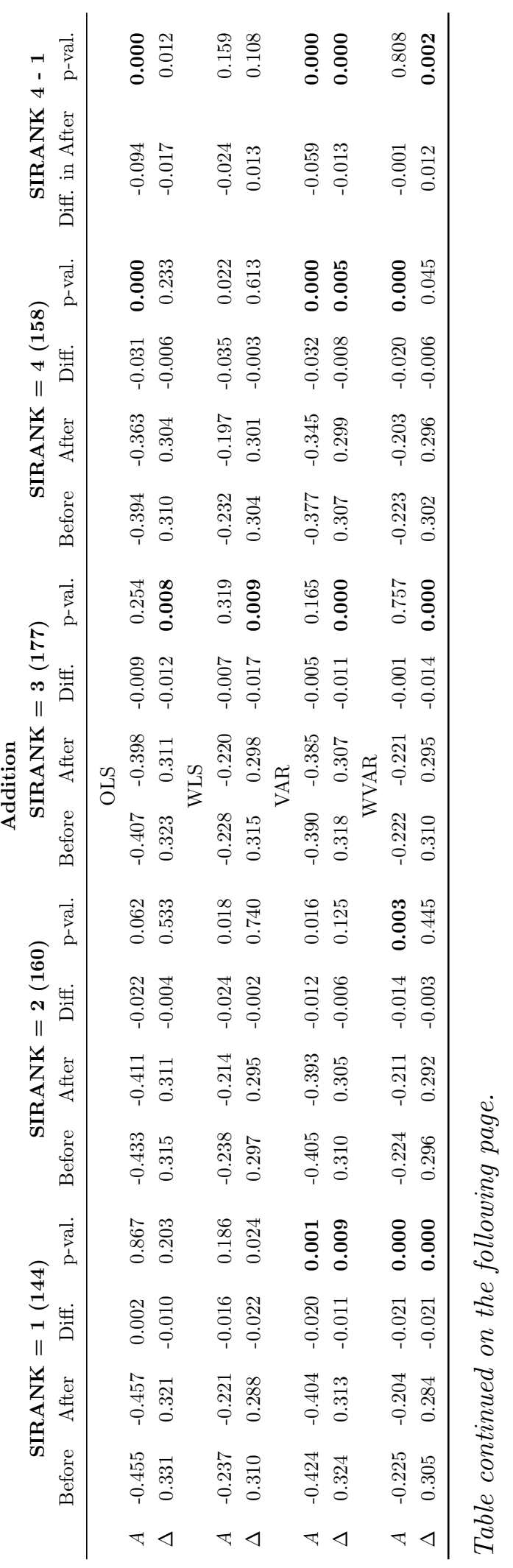




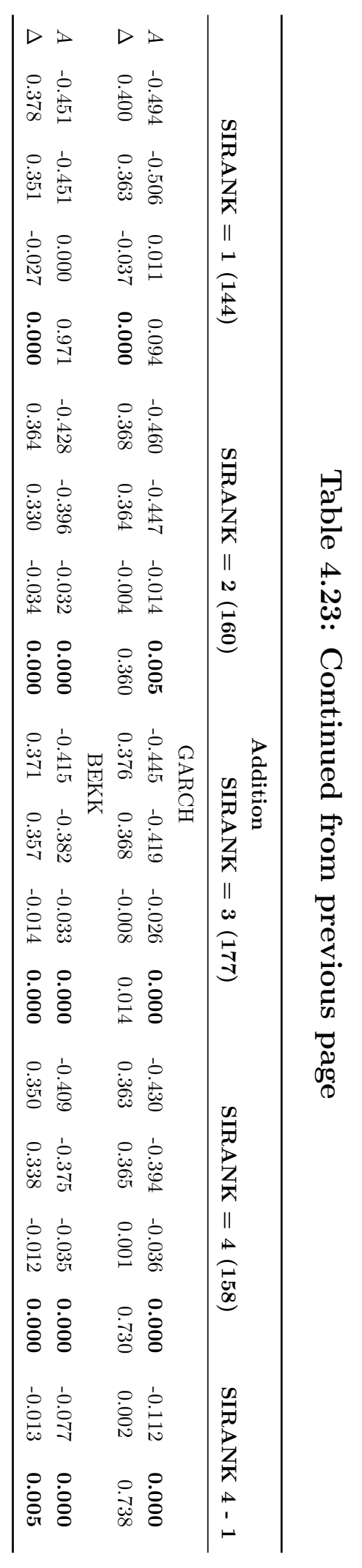




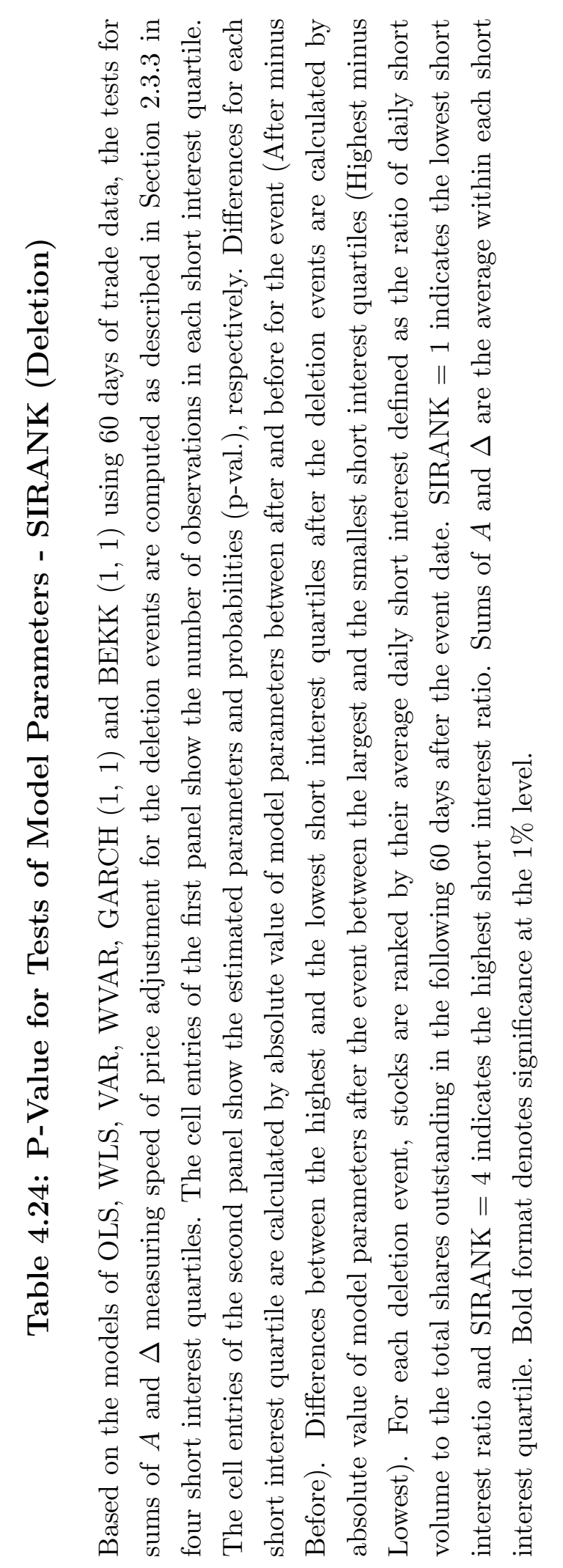

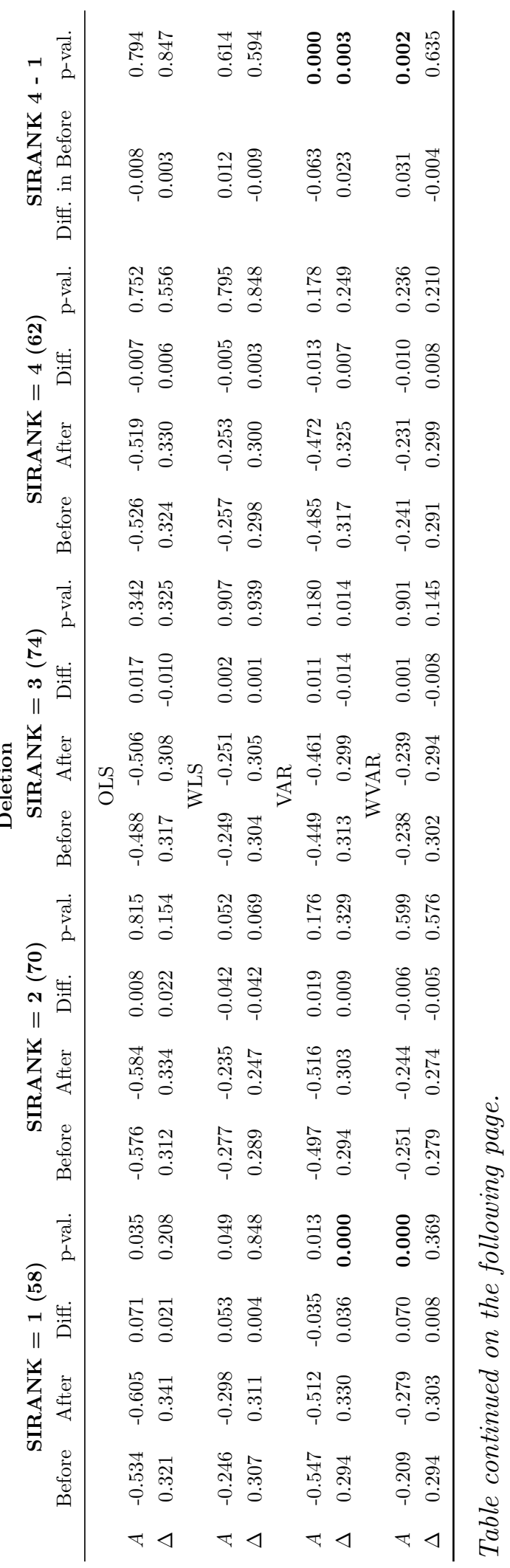




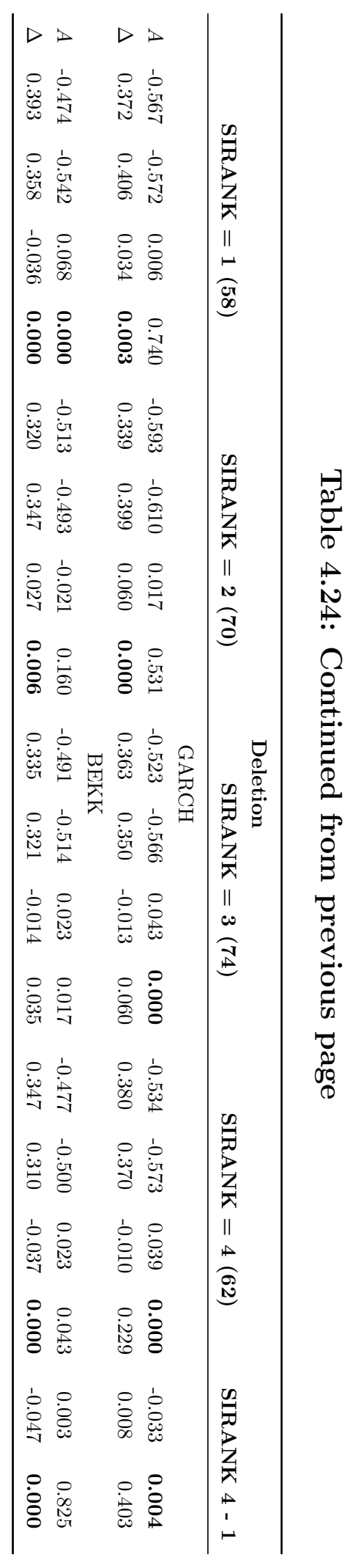




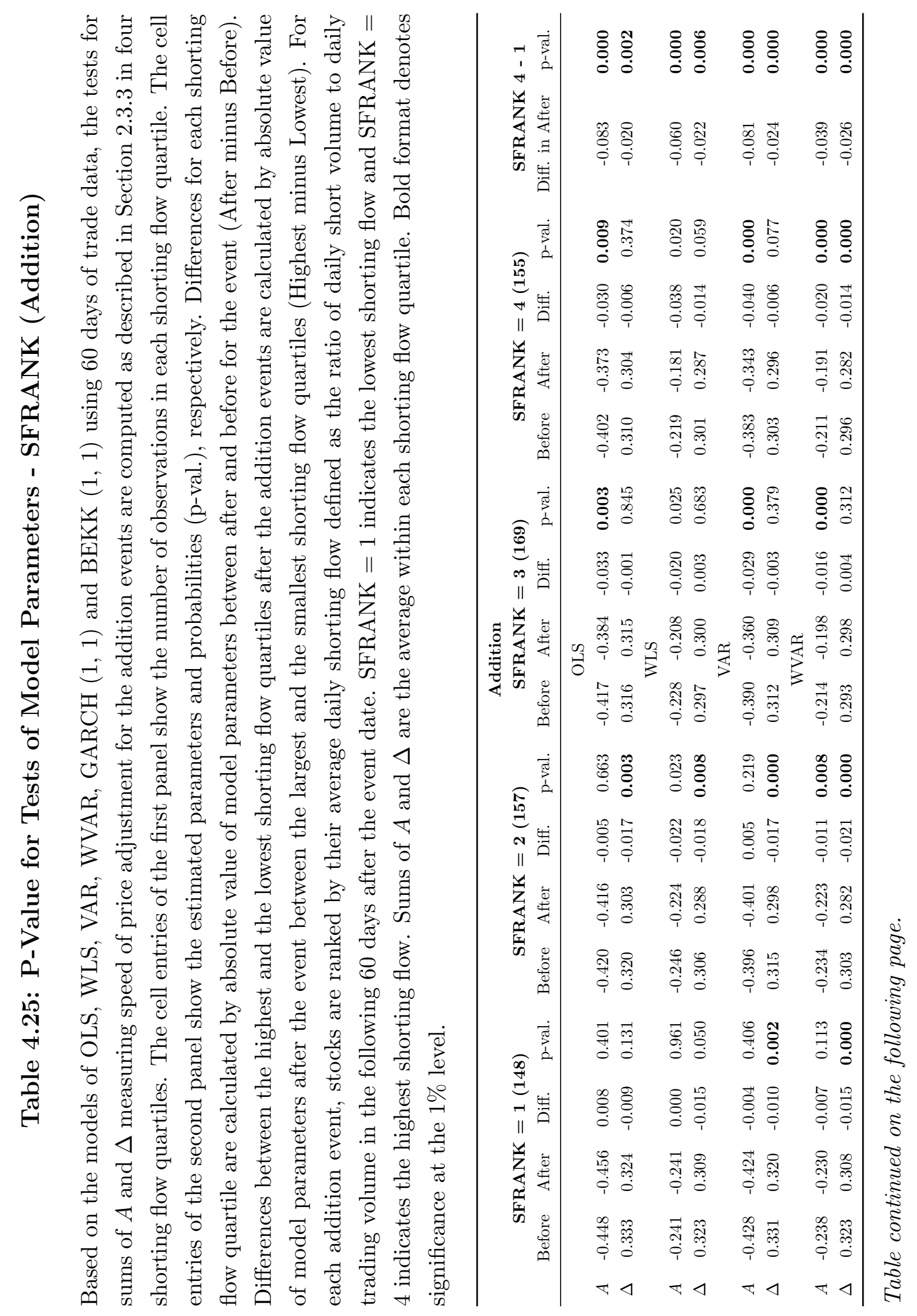




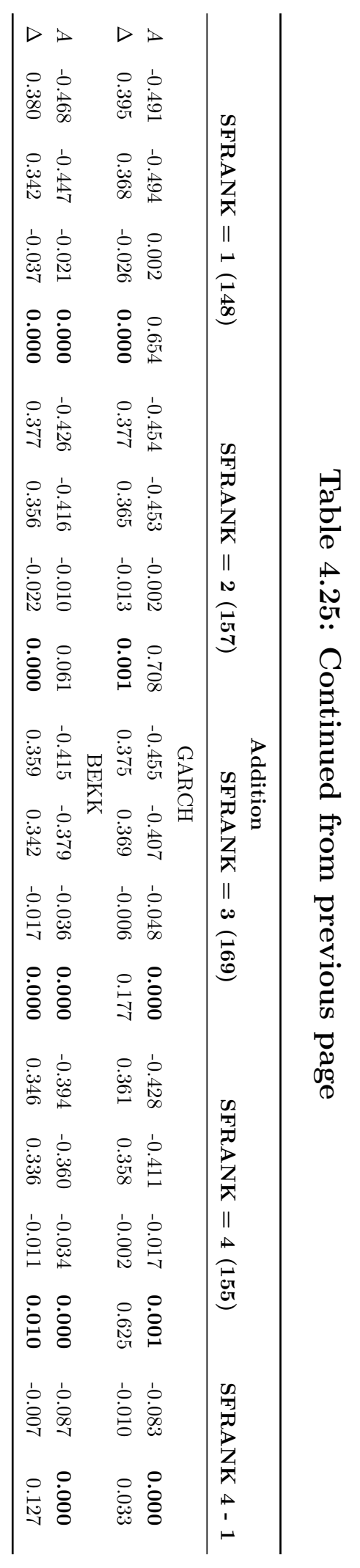




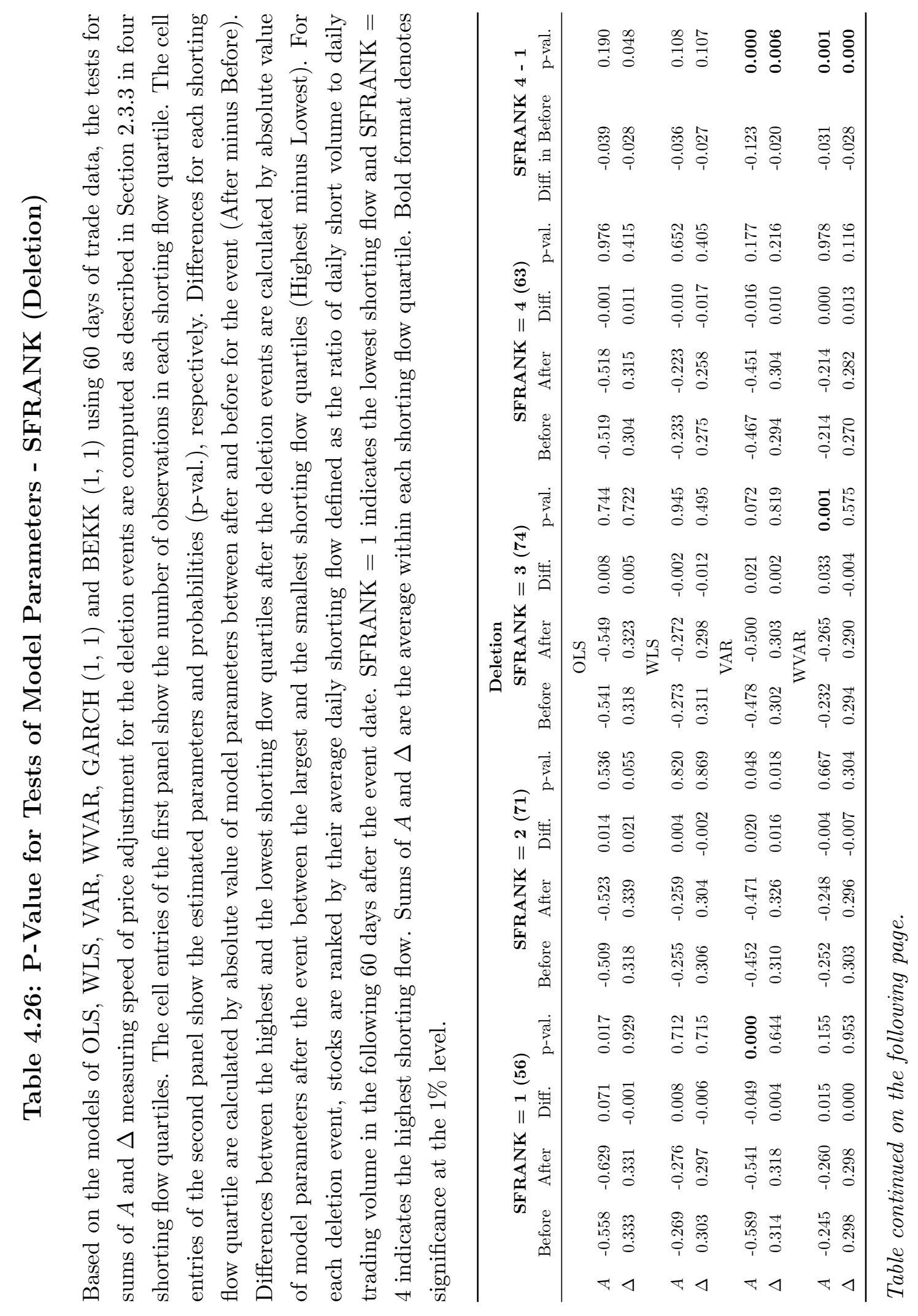




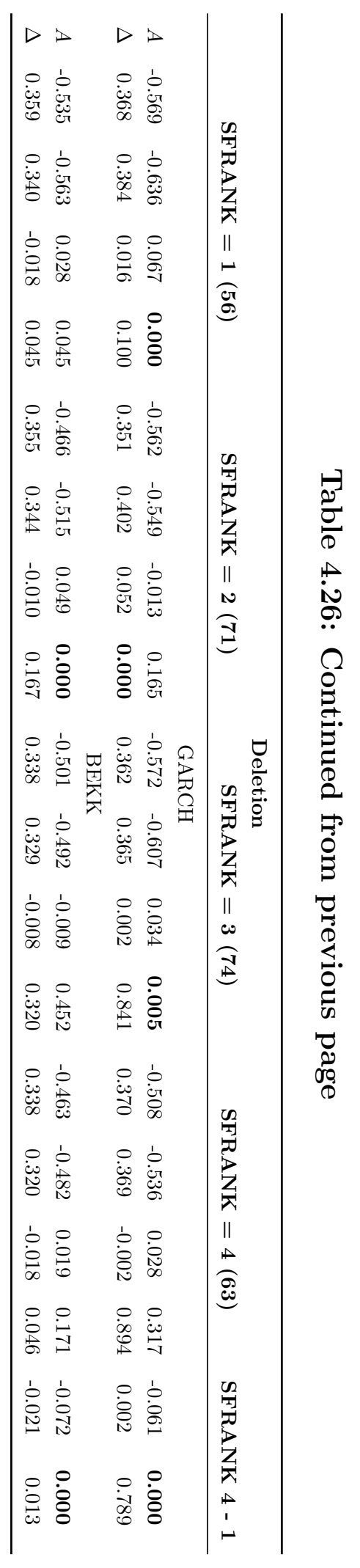


Appendix F

Other Table 


\section{Table F.1: Cumulative Abnormal Returns Before, During and After Crisis.}

The table reports the cumulative abnormal returns based on the market model estimated before, during and after the crisis. The period of crisis is defined as the period from December 2007 to June 2009. For the market model, an estimation window of $(-240,-60)$, with a minimum length of 180 days, is used. Each panel of the table indicates that the minimum length of time for stocks to be added back after deletion from the D-list.

\begin{tabular}{|c|c|c|c|c|c|c|}
\hline \multicolumn{7}{|c|}{ Cumulative Daily Abnormal Returns (\%) } \\
\hline \multirow{2}{*}{ Event Windows } & \multicolumn{2}{|c|}{ Before Crisis } & \multicolumn{2}{|c|}{ During Crisis } & \multicolumn{2}{|c|}{ After Crisis } \\
\hline & Mean & $\mathrm{p}$-value & Mean & $\mathrm{p}$-value & Mean & $\mathrm{p}$-value \\
\hline \multicolumn{7}{|c|}{ 1-Year } \\
\hline$(0,10)$ & 2.24 & 0.01 & 1.97 & 0.15 & -2.74 & 0.03 \\
\hline$(0,30)$ & 2.62 & 0.08 & 8.35 & 0.00 & -4.20 & 0.09 \\
\hline$(0,60)$ & 4.34 & 0.06 & 18.58 & 0.00 & -3.60 & 0.32 \\
\hline \multicolumn{7}{|c|}{ 2-Year } \\
\hline$(0,10)$ & 1.74 & 0.03 & 0.89 & 0.40 & -3.22 & 0.04 \\
\hline$(0,30)$ & 2.30 & 0.13 & 6.77 & 0.00 & -5.47 & 0.06 \\
\hline$(0,60)$ & 5.10 & 0.03 & 17.66 & 0.00 & -5.68 & 0.18 \\
\hline \multicolumn{7}{|c|}{3 -Year } \\
\hline$(0,10)$ & 1.52 & 0.08 & 0.45 & 0.63 & -4.09 & 0.06 \\
\hline$(0,30)$ & 2.78 & 0.07 & 5.17 & 0.00 & -7.03 & 0.04 \\
\hline$(0,60)$ & 5.26 & 0.05 & 15.92 & 0.00 & -8.17 & 0.11 \\
\hline
\end{tabular}




\section{Chapter 5}

\section{Conclusions}

This thesis investigates whether short sales in the Hong Kong stock market aid price efficiency as measured as the speed of price adjustment to new information using ultra-high frequency trade-by-trade data over 10 years from 2001 to 2011. The selection criteria for short sales in Hong Kong provides a unique opportunity to explore the impact of short sales on price efficiency as the market only allows stocks which meet certain requirements to be added to the list for short selling. Furthermore, this list is revised by the stock exchange every three months. This setting ensures that any differences in the speed of price adjustment in the three months before and after each addition and deletion event can be directly linked to the changes in the eligibility for short sales. The thesis addresses three distinct but related topics. In what follows, it presents a brief summary of the empirical findings and highlights potential areas for future research.

\subsection{Summary of Findings}

Chapter 2 extends the bivariate VAR model of Hasbrouck (1991) and offers a direct examination of the effect of short sales on price efficiency in the microstructure model for changes in quotes and trade dynamics. In addition to the use of least squares and weighted least squares on an equation by equation basis, a bona fide 
VAR model is estimated simultaneously with consideration of the cross-sectional correlation of the residuals in the two equations. Therefore, four different estimation methods namely OLS, WLS, VAR and WVAR are included in this chapter.

The main findings of Chapter 2 are as follows. First, there is a subset of stocks with significant changes including increases and decreases in model parameters as the proxies for price efficiency for both addition and deletion events. Based on the results under four estimation models, it is noted that there are more significant changes captured in individual and aggregated parameters during both events when the models are either estimated simultaneously or without consideration of time duration of trades. In other words, it indicates that the VAR and WVAR models are more sensitive in capturing significant changes than their least squares counterparts while time-weighted models (WLS and WVAR) are more conservative.

Secondly, the study of parameter dynamics under four models show that the model parameters, except for those measuring Granger causality, largely remain consistent during both events. It shows that positive/negative parameters which are statistically significant will generally stay in the same significance category with the same sign, even if the estimated values change as a result of the event. Furthermore, it is found that the model parameters are less consistent under the time-weighted models and the consistency is greater for the addition stocks rather than the deletion stocks.

Thirdly, the chapter carries out an investigation on the overall effect of short sales on price efficiency as the significant changes captured in parameters include both increased and decreased values. The $Z$ scores for tests of differences in model parameters show that the autocorrelations in both quote returns and trades decrease significantly during the addition events and the autocorrelations in quote returns increase significantly during the deletion events. The overall results for both events suggest that the speed of price adjustment is faster with the eligibility of shorts sales as a smaller absolute value of autocorrelation in quote returns and trades indicate that stock prices follow a random walk more closely. It is also found that 
trade continuity (autocorrelation in quote returns) is affected the most during the addition (deletion) events under four different models. Furthermore, the price impact of trades get stronger when stocks are added to the D-list for short selling under models when the time duration between consecutive transactions is considered.

Fourthly, this chapter also provides some indications of the best model based on the statistical tests on residual correlations and a summary of model estimations under OLS, WLS, VAR and WVAR. Based on residual correlation tests, VAR and WVAR models have better ability to capture residual correlations between quote and trade equations than OLS and WLS models that are estimated equation by equation. Based on the summary of model estimations, more significant improvements (deteriorations) in price efficiency are captured for the addition (deletion) events under VAR and WVAR models than OLS and WLS models. It suggests that VAR and WVAR models are more powerful to explore the differences in the speed of price adjustment during both events. There is no direct standard test to the best of our knowledge to compare between models with and without time-weighted variables. Therefore, the WVAR model is selected to be the preferred model as it is more rigorous theoretically because trades do not occur at regularly spaced time intervals.

The ultra-high frequency based quotations and signed volumes invariably exhibit conditional heteroscedasticity and it is conjectured that heterogeneous variances and covariances could have an effect on the model parameters. Chapter 3 extends the VAR model by conducting the analysis using GARCH and BEKK models. Univariate GARCH $(1,1)$ models capture dynamic volatility in each equation while the $\operatorname{BEKK}(1,1)$ model considers the co-movement and non-independence of volatility between quote returns and trades.

The findings of Chapter 3 are summarised as follows. First, a number of stocks exhibit significant changes for addition and deletion events under GARCH and BEKK models. Compared with the results from OLS and VAR models, there are 
more significant changes captured in all corresponding parameters under the models with consideration of time-varying variances and covariances. It reveals that the models taking conditional heteroscedasticity into account have a deeper insight into the effect of short sales on the model parameters for both events. However, there are still less than $40 \%$ (30\%) of the stocks affected significantly by changes in the parameters measuring the speed of price adjustment during the addition (deletion) events. Taken together, it means that less than half of the stocks contribute to the overall changes in the speed of price adjustment under all estimation models.

Secondly, the study of parameter dynamics shows that the majority of the model parameters, except for Granger causality, are consistent when stocks change their eligibility of short sales. Moreover, it is interesting to note that the parameter consistency is stronger especially for the autocorrelations in quote returns and trades (the sums, $A$ and $\Delta$ ) under GARCH model rather than OLS while the consistency is qualitatively the similar between VAR and BEKK.

Thirdly, the overall results from $Z$ scores for tests of difference in model parameters for both events show that gaining (losing) the eligibility of short sales accelerate (hinder) the speed of price adjustment by decreasing (increasing) the autocorrelation in the parameters measuring price efficiency. The findings are consistent with those from Chapter 2. The combined results from Chapters 2 and 3 indicate that trade continuity decreases the most by short sales with robustness under all estimation models. Quote returns are less correlated at significance level 1\% under both WVAR and BEKK during the addition events, this finding being consistent with Boehmer \& Wu (2013)'s prediction that an efficient price process is associated with a random walk if the quotations are the best estimate of the equilibrium value of the stocks in the market. For the deletion events, autocorrelations in quote returns is strengthened the most and stronger stickiness in quote returns indicates the delayed price incorporation to new information when stocks are removed from the D-list. Trade continuity is not affected significantly during the deletion events. In sum, the results support that the efficiency-enhancing effect of short sales is economically meaningful. Lastly, it is also noted that the models estimated simultaneously or with consideration of heterogeneous variances, that is WVAR and 
BEKK models, are more powerful to capture the differences in the speed of price adjustment especially during the addition events.

Chapter 4 carries on an investigation of the determinants of the speed of price adjustment including market conditions, firm size, trading volume and short sellers' activities during both addition and deletion events.

The results of Chapter 4 are as follows. To explore the speed of price adjustment under various market conditions during both events, the chapter introduces three different methods: models with interaction variables for current and oneday lagged market return; comparison between crisis and non-crisis periods; reestimation based on the positive and non-positive transaction days, respectively. The overall results show that short sales speed up the price adjustment regardless of market conditions. Stocks have a faster speed of incorporation of new information into prices in a down or bad market rather than in an up or good market. By comparing the crisis and the non-crisis periods, it is found that short sales aid price efficiency in the non-crisis period whereas the effect of short sales is unclear during in the crisis.

By dividing the estimation results from Chapters $2 \& 3$ into four-sized groups based on their 60-day average market value prior to the effective date, it is found that bigger-sized firms have faster speed of price adjustment to new information than smaller-sized ones and the results are robust under all estimation models. It is also interesting to note that the medium-sized (small-sized) firms are the most affected when they are added to (removed from) the D-list for short sales. Comparing stocks with different trading volumes, the results show that stocks with higher trading volume are more likely to be influenced by short sales during the addition events while stocks from the top and the bottom are impacted most during the deletion events. Stocks with more trading volumes are faster to adjust their prices compared with those with lower trading volume. Two proxies for short sales employed to investigate the role of short sellers' activities in the process of price discovery. The findings show that in general stocks with higher short interest have 
better price efficiency especially under the models without time durations while stocks with more shorting flow experience a faster speed of price adjustment to new information. These two measures reveal that short sellers contribute significantly to an efficient price discovery and the findings support the hypothesis that short sellers are informed traders with adverse information which can help to correct prices back to their fundamental levels (Boehmer et al., 2008; Desai et al., 2002; Diether et al., 2009).

This thesis investigates the role of short sales in the process of price discovery in the Hong Kong market where the institutional environment provides a rare opportunity with a designated list for short sales. There are 808 stocks with changes of eligibility of short sales over a 10-year period. Among them, there are $72 \mathrm{H}$-shares and 80 red chips stocks, totally 152 out of 808 stocks (approximately 20\%) with mainland Chinese background. $\mathrm{H}$ shares refer to the shares of companies incorporated in mainland China that are traded on the HKEx. Red chips stocks are the stocks of mainland China companies incorporated outside mainland China and listed on the HKEx. The businesses of red chips stocks are based in mainland China and controlled, either directly or indirectly, by the central, provincial or municipal governments of China but listed in Hong Kong to allow overseas investment in the companies.

The China Securities Regulatory Commission (CSRC) introduced a pilot program of margin trading in 2010, allowing 90 stocks quoted on either the Shanghai or Shenzhen stock exchanges on a designated list to be sold short and/or purchased on margin. Since the end of the pilot programme, over 800 quoted Chinese stocks have become eligible for short selling in both Chinese stock markets. Given 20\% of event stocks with mainland Chinese background, results from the thesis may provide information about the possible impact of short sales on price efficiency in mainland China and provide guidance for policy making for Chinese regulators to facilitate the further development of the market. 


\subsection{Areas for Future Research}

This study has focused on the effect of short selling on price efficiency. The lifting of short sales constraints contributes to the speed of price adjustment by the participation of pessimistic informed investors. The resolution of uncertainty about the fundamentals tends to improve stock liquidity by decreasing the bid-ask spread (Diamond \& Verrecchia, 1987). Short sellers could, however, also impact stock liquidity in the opposite way. Short sellers will lower stock liquidity because they, as informed traders, are more likely to be liquidity demanders rather than liquidity suppliers in the market (Boehmer et al., 2008). Moreover, a large volume of informed trading based on private information will force market makers to increase the adverse selection cost of bid-ask spread for compensation, thus resulting in the reduction of stock liquidity (Amihud \& Mendelson, 1986). Future research can be conducted on the effect of short sales on liquidity, based upon the models developed in this thesis. Further research to identify more detailed explanations for deletion events during the financial crisis period would also be of interest. This is because the initial investigations reported in Chapter 4 suggest that during the crisis deletion events served as a proxy for a partial short sales ban and so serve a genuine economic function.

This thesis makes several contributions to both the existing literature and financial practice. It also has some limitations which are directions for future research.

First, the dataset used for this study is from May 2001 to May 2011. Although a 10-year intraday dataset is sufficient to examine the effect of short sales on the speed of price adjustment in detail, a more recent dataset would add to the robustness of the research. Specifically, it would permit the investigation of $(i)$ possible parameter changes and ( $i i)$ the extent to which the models developed in this thesis could be used for forecasting purposes.

Secondly, in Chapter 4, the Hang Seng daily market index is used to construct the dummy variable of market condition in the VAR model interacted with model 
parameters to investigate the process of price discovery under different market conditions. As discussed in that chapter, the dummy variable of current market return used in the model raises foresight issues as quotations and trades at an arbitrary time of the day are associated with the dummy variable calculated by the price at the end of the day. Although the dummy variable of one-day lagged market return is employed to solve this issue, high frequency market index data would be more appropriate to use with quotes and trades in the model.

Thirdly, the models use a variable based on price quotations. This is similar to returns defined in the conventional manner but is not exactly the same. From a theoretical perspective it would be instructive to determine to what extent these non-standard returns are consistent with standard asset pricing models.

Lastly, results from Chapters $2 \& 3$ show that the WVAR and BEKK models are more powerful to capture the changes in the speed of price adjustment compared with other models. However, there is no clear evidence to support the exclusive use of one model in preference to another. Furthermore, particularly at the aggregate level, the differences between all six models reported in this thesis are not great. An updated data set would facilitate further analysis of model differences. As noted in Chapter 4, there is a need for development of model software. As noted in Chapter 2 , there is a need for development of statistical tests. Distinguishing the differences between the two estimation methods in the microstructure models can contribute to model selection for high frequency trading strategies in future research. 


\section{References}

Aminud, Y. \& Mendelson, H. (1986). Asset pricing and the bid-ask spread. Journal of Financial Economics, 17, 223-249.

Bauwens, L., Laurent, S. \& Rombouts, J.V.K. (2006). Multivariate GARCH models: A survey. Journal of Applied Econometrics, 21, 79-109.

Beber, A. \& Pagano, M. (2013). Short-selling bans around the world: Evidence from the 2007-09 crisis. The Journal of Finance, 68, 343-381.

BerA, A.K. \& Jarque, C.M. (1982). Model specification tests. Journal of Econometrics, 20, 59-82.

Boenmer, E. \& Wu, J. (2013). Short selling and the price discovery process. Review of Financial Studies, 26, 287-322.

Boehmer, E., Jones, C.M. \& Zhang, X.Y. (2008). Which shorts are informed ? Journal of Finance, 63, 491-527.

Bollerslev, T. (1986). Generalized autoregressive conditional heteroskedasticity. Journal of econometrics, 31, 307-327.

Bollerslev, T. \& Wooldridge, J.M. (1992). Quasi-maximum likelihood estimation and inference in dynamic models with time-varying covariances. Econometric reviews, $\mathbf{1 1}, 143-172$.

Bollerslev, T., Engle, R.F. \& Wooldridge, J.M. (1988). A capital-asset pricing model with time-varying covariances. Journal of Political Economy, 96, $116-131$. 
Breusch, T.S. \& Pagan, A.R. (1979). A simple test for heteroscedasticity and random coefficient variation. Econometrica, 47, 1287-1294.

Bris, A., Goetzmann, W.N. \& Zhu, N. (2007). Efficiency and the bear: Short sales and markets around the world. Journal of Finance, 62, 1029-1079.

Brown, K.C., Harlow, W. \& Tinic, S.M. (1988). Risk aversion, uncertain information, and market efficiency. Journal of Financial Economics, 22, 355385 .

Chang, E.C., Cheng, J.W. \& Yu, Y.H. (2007). Short-sales constraints and price discovery: Evidence from the Hong Kong market. Journal of Finance, 62, $2097-2121$.

Chang, E.C., Luo, Y. \& Ren, J.J. (2014). Short-selling, margin-trading, and price efficiency: Evidence from the Chinese market. Journal of Banking \& Finance, 48, 411-424.

Chen, C.X. \& Rhee, S.G. (2010). Short sales and speed of price adjustment: Evidence from the Hong Kong stock market. Journal of Banking $\mathscr{E}$ Finance, 34, 471-483.

Chen, J., Hong, H. \& Stein, J.C. (2002). Breadth of ownership and stock returns. Journal of Financial Economics, 66, 171-205.

Chordia, T. \& Swaminathan, B. (2000). Trading volume and crossautocorrelations in stock returns. Journal of Finance, 55, 913-935.

Chordia, T., Roll, R. \& Subrahmanyam, A. (2005). Evidence on the speed of convergence to market efficiency. Journal of Financial Economics, 76, 271-292.

Chung, K.H., Li, M.S. \& McInish, T.H. (2005). Information-based trading, price impact of trades, and trade autocorrelation. Journal of Banking \& Finance, 29, 1645-1669.

Danielsen, B.R. \& Sorescu, S.M. (2001). Why do option introductions depress stock prices? A study of diminishing short sale constraints. The Journal of Financial and Quantitative Analysis, 36, 451-484. 
Desai, H., Ramesh, K., Thiagarajan, S.R. \& Balachandran, B.V. (2002). An investigation of the informational role of short interest in the Nasdaq market. Journal of Finance, 57, 2263-2287.

Diamond, D.W. \& Verrecchia, R.E. (1987). Constraints on short-selling and asset price adjustment to private information. Journal of Financial Economics, 18, 277-311.

Diether, K.B., Lee, K.H. \& Werner, I.M. (2009). Short-sale strategies and return predictability. Review of Financial Studies, 22, 575-607.

Dimson, E. (1979). Risk measurement when shares are subject to infrequent trading. Journal of Financial Economics, 7, 197-226.

Dufour, A. \& Engle, R.F. (2000). Time and the price impact of a trade. Journal of Finance, 55, 2467-2498.

Easley, D. \& O'Hara, M. (1992). Time and the process of security price adjustment. The Journal of Finance, 47, 577-605.

Easley, D., Kiefer, N.M. \& O'Hara, M. (1997). One day in the life of a very common stock. Review of Financial Studies, 10, 805-835.

Engle, R. (2002). Dynamic conditional correlation: A simple class of multivariate generalized autoregressive conditional heteroskedasticity models. Journal of Business \& Economic Statistics, 20, 339-350.

Engle, R.F. (1982). Autoregressive conditional heteroscedasticity with estimates of the variance of United Kingdom inflation. Econometrica: Journal of the Econometric Society, 987-1007.

Engle, R.F. \& Bollerslev, T. (1986). Modelling the persistence of conditional variances. Econometric Reviews, 5, 1-50.

Engle, R.F. \& Kroner, K.F. (1995). Multivariate simultaneous generalized ARCH. Econometric Theory, 11, 122-150. 
Engle, R.F., Lilien, D.M. \& Robins, R.P. (1987). Estimating time varying risk premia in the term structure: The ARCH-M model. Econometrica, 55, 391407.

FAmA, E.F. (1991). Efficient capital markets: II. The Journal of Finance, 46, $1575-1617$.

Figlewski, S. (1981). The informational effects of restrictions on short sales: Some empirical evidence. Journal of Financial and Quantitative Analysis, 16, 463-476.

Foerster, S.R. \& Karolyi, G.A. (1999). The effects of market segmentation and investor recognition on asset prices: Evidence from foreign stocks listing in the United States. The Journal of Finance, 54, 981-1013.

Hasbrouck, J. (1991). Measuring the information-content of stock trades. Journal of Finance, 46, 179-207.

Hasbrouck, J. (1993). Assessing the quality of a security market: A new approach to transaction-cost measurement. Review of Financial Studies, 6, 191-212.

Hasbrouck, J. \& Ho, T.S.Y. (1987). Order arrival, quote behavior, and the return-generating process. The Journal of Finance, 42, 1035-1048.

Hosking, J.R.M. (1980). The multivariate portmanteau statistic. Journal of the American Statistical Association, 75, 602-608.

Hou, K. \& Moskowitz, T.J. (2005). Market frictions, price delay, and the cross-section of expected returns. Review of Financial Studies, 18, 981-1020.

Jones, C.M. \& Lamont, O.A. (2002). Short-sale constraints and stock returns. Journal of Financial Economics, 66, 207-239.

Kahneman, D. \& Tversky, A. (1979). Prospect theory: An analysis of decision under risk. Econometrica: Journal of the Econometric Society, 263-291.

LEe, C.M.C. \& READY, M.J. (1991). Inferring trade direction from intraday data. Journal of Finance, 46, 733-746. 
Lo, A.W. \& MacKinlay, A.C. (1988). Stock market prices do not follow random walks: Evidence from a simple specification test. Review of Financial Studies, 1, 41-66.

Lo, A.W. \& MacKinlay, A.C. (1990). When are contrarian profits due to stock market overreaction? Review of Financial Studies, 3, 175-205.

Madhavan, A., Richardson, M. \& Roomans, M. (1997). Why do security prices change? A transaction-level analysis of NYSE stocks. Review of Financial Studies, 10, 1035-1064.

MAndelbrot, B. (1963). The variation of certain speculative prices. The Journal of Business, 36, 394-419.

MCLEOD, A.I. \& LI, W.K. (1983). Diagnostic checking ARMA time series models using squared-residual autocorrelations. Journal of Time Series Analysis, 4, 269 273.

Miller, E.M. (1977). Risk, uncertainty, and divergence of opinion. Journal of Finance, 32, 1151-1168.

Morck, R., Yeung, B. \& Yu, W. (2000). The information content of stock markets: Why do emerging markets have synchronous stock price movements? Journal of Financial Economics, 58, 215-260.

NAGEL, S. (2005). Short sales, institutional investors and the cross-section of stock returns. Journal of Financial Economics, 78, 277-309.

Nelson, D.B. (1991). Conditional heteroskedasticity in asset returns: A new approach. Econometrica, 59, 347-370.

Saffi, P.A.C. \& Sigurdsson, K. (2010). Price efficiency and short selling. Review of Financial Studies, 24, 821-852.

Scotт, A.J. \& Smith, T.M.F. (1971). Interval estimates for linear combinations of means. Journal of the Royal Statistical Society Series C-Applied Statistics, 20, 276-285. 
Sortino, F.A. \& Van Der Meer, R. (1991). Downside risk. The Journal of Portfolio Management, 17, 27-31.

White, H. (1980). A heteroskedasticity-consistent covariance-matrix estimator and a direct test for heteroskedasticity. Econometrica, 48, 817-838.

ZakoiAn, J.M. (1994). Threshold heteroskedastic models. Journal of Economic Dynamics and Control, 18, 931-955. 